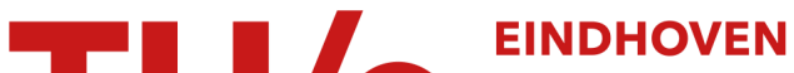 \\ UNIVERSITY OF \\ TECHNOLOGY
}

\section{Stress induced crystallization in elongational flow}

Citation for published version (APA):

Swartjes, F. H. M. (2001). Stress induced crystallization in elongational flow. [Phd Thesis 1 (Research TU/e / Graduation TU/e), Mechanical Engineering]. Technische Universiteit Eindhoven.

https://doi.org/10.6100//R549524

DOI:

10.6100/IR549524

Document status and date:

Published: 01/01/2001

\section{Document Version:}

Publisher's PDF, also known as Version of Record (includes final page, issue and volume numbers)

\section{Please check the document version of this publication:}

- A submitted manuscript is the version of the article upon submission and before peer-review. There can be important differences between the submitted version and the official published version of record. People interested in the research are advised to contact the author for the final version of the publication, or visit the $\mathrm{DOI}$ to the publisher's website.

- The final author version and the galley proof are versions of the publication after peer review.

- The final published version features the final layout of the paper including the volume, issue and page numbers.

Link to publication

\section{General rights}

Copyright and moral rights for the publications made accessible in the public portal are retained by the authors and/or other copyright owners and it is a condition of accessing publications that users recognise and abide by the legal requirements associated with these rights.

- Users may download and print one copy of any publication from the public portal for the purpose of private study or research.

- You may not further distribute the material or use it for any profit-making activity or commercial gain

- You may freely distribute the URL identifying the publication in the public portal.

If the publication is distributed under the terms of Article 25fa of the Dutch Copyright Act, indicated by the "Taverne" license above, please follow below link for the End User Agreement:

www.tue.nl/taverne

Take down policy

If you believe that this document breaches copyright please contact us at:

openaccess@tue.nl

providing details and we will investigate your claim. 


\section{Stress Induced Crystallization in Elongational Flow}


CIP-DATA LIBRARY TECHNISCHE UNIVERSITEIT EINDHOVEN

Swartjes, Frank H.M.

Stress induced crystallization in elongational flow / by Frank H.M. Swartjes. Eindhoven : Technische Universiteit Eindhoven, 2001.

Proefschrift. - ISBN 90-386-3052-2

NUGI 841

Trefwoorden: polymeren; reologie / stromingsgeïnduceerde kristallisatie / fysischchemische simulatie en modellering; contitutieve vergelijkingen / grote hoek röntgenverstrooiing

Subject headings: polymers; rheology / flow-induced crystallization / physicochemical simulation and modeling; constitutive equations / wide-angle X-ray scattering (WAXS)

Printed by the University Press Facilities, Eindhoven, the Netherlands.

This research was financially supported by the Dutch Polymer Institute (DPI; project \#128) and by the European Commission (BRITE-EURAM III project BE 95-2067). 


\title{
Stress Induced Crystallization in Elongational Flow
}

\author{
PROEFSCHRIFT \\ ter verkrijging van de graad van doctor aan de \\ Technische Universiteit Eindhoven, op gezag van de \\ Rector Magnificus, prof.dr. R.A. van Santen, voor een \\ commissie aangewezen door het College voor \\ Promoties in het openbaar te verdedigen \\ op 8 november 2001 om 16.00 uur
}

door

Franciscus Henrikus Martinus Swartjes

geboren te Beuningen (Gld) 
Dit proefschrift is goedgekeurd door de promotoren:

prof.dr.ir. H.E.H. Meijer

en

prof.dr. P.J. Lemstra

Copromotor:

dr.ir. G.W.M. Peters 


\section{Contents}

Summary vii

1 Introduction 1

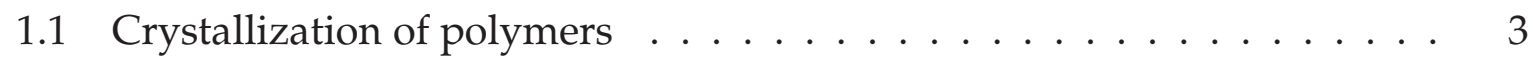

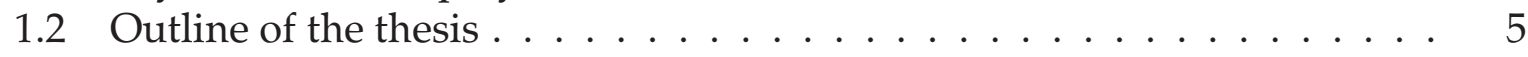

2 Methods and materials $\quad 7$

2.1 Experimental methods . . . . . . . . . . . . . . . . 7

2.1 .1 DSC ......................... 7

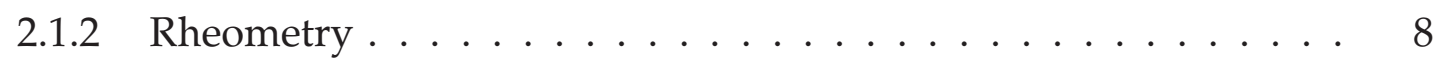

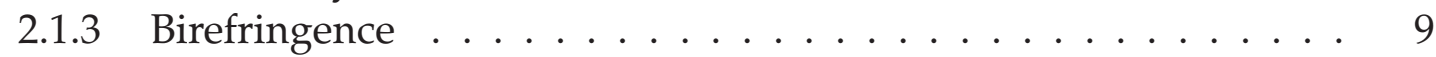

2.1 .4 Microscopy . . . . . . . . . . . . . . . . 12

2.1 .5 X-ray scattering . . . . . . . . . . . . . . . 12

2.2 Materials selection: iPP . . . . . . . . . . . . . . . . . 15

2.3 Measurements of flow-induced nucleation and crystallization . . . . . . 23

2.4 Discussion ... . . . . . . . . . . . . . . . . . 29

3 Constitutive models $\quad 31$

3.1 Constitutive equations for the stress tensor . . . . . . . . . . . . 31

3.1.1 Generalized Newtonian model . . . . . . . . . . . . . . . . 31

3.1.2 Nonlinear viscoelastic models . . . . . . . . . . . . . 32

3.2 Constitutive equations for flow-induced nucleation and crystallization . 36

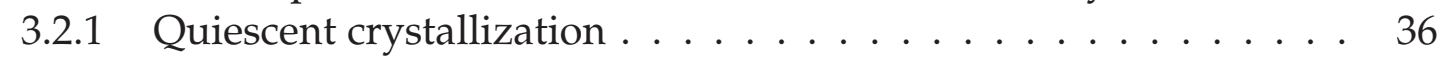

3.2.2 Flow-induced nucleation and crystallization . . . . . . . . . . . 37

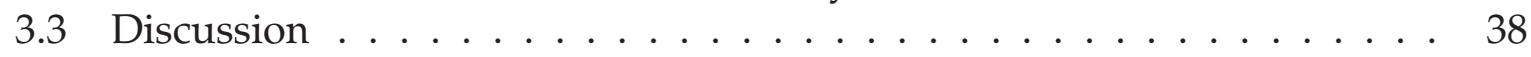

4 Materials characterization 39

4.1 Introduction . . . . . . . . . . . . . . . . . . 39

4.2 Rheological characterization . . . . . . . . . . . . . . . . . 40

4.2.1 High molecular weight (DSM13E10) . . . . . . . . . . . . . . 43

4.2.2 Low molecular weight (DSM15M10) . . . . . . . . . . . . 44

4.3 Determination of $\mathrm{G}(\mathrm{T})$ and $\mathrm{N}(\mathrm{T}) \ldots \ldots \ldots \ldots$. . . . . . . . . . . 44

4.3 .1 Determination of $\mathrm{G}(\mathrm{T}) \ldots \ldots \ldots \ldots$

4.3 .2 Determination of $\mathrm{N}(\mathrm{T}) \ldots \ldots \ldots \ldots$

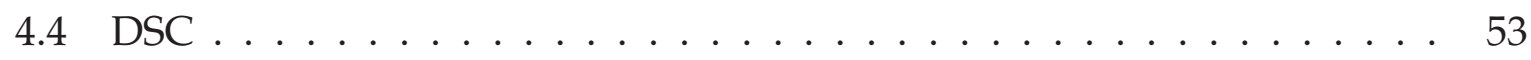

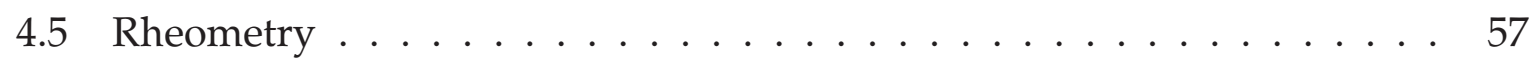




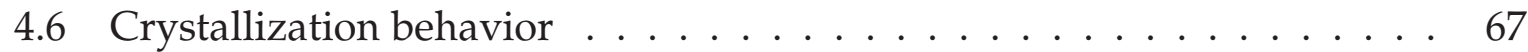

4.6.1 Fitting of the SOAS experiments . . . . . . . . . . . . 67

4.6.2 Influence of nucleation on rheology . . . . . . . . . . . . 69

4.7 Discussion . . . . . . . . . . . . . . . . . . 73

5 Nucleation and crystallization in a cross-slot flow cell: Numerical analysis 75

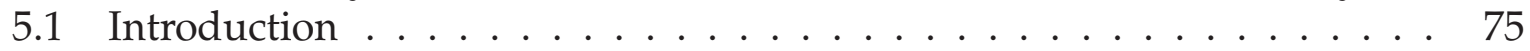

5.2 Numerical methods . . . . . . . . . . . . . . . . . . . 75

5.3 Numerical results . . . . . . . . . . . . . . . . . . . . . . . . . . . . 79

5.4 Discussion . . . . . . . . . . . . . . . . . . . 82

6 Nucleation and crystallization in a cross-slot flow cell: Experiments and pre$\begin{array}{lr}\text { dictions } & 83\end{array}$

6.1 Introduction . . . . . . . . . . . . . . . . . 83

6.2 Cross slot flow cell . . . . . . . . . . . . . . . . . 83

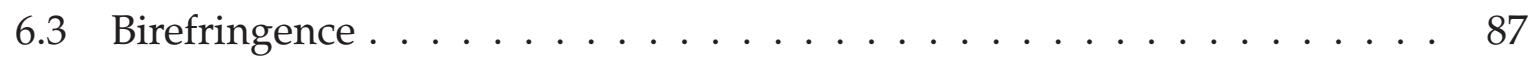

6.3 .1 Experimental set-up . . . . . . . . . . . . 87

6.3 .2 Experimental results . . . . . . . . . . . . . . . . 88

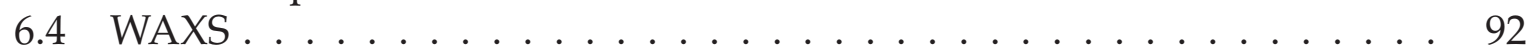

6.4.1 Experimental set-ups . . . . . . . . . . . . . . . 92

6.4 .2 Experimental results . . . . . . . . . . . . . 93

6.5 Numerical predictions . . . . . . . . . . . . . . . . . . . . . 97

6.6 Discussion . . . . . . . . . . . . . . . . . . . 101

7 Conclusions and recommendations 103

7.1 Conclusions . . . . . . . . . . . . . . . . . 103

7.2 Recommendations ....................... 105

$\begin{array}{ll}\text { References } & 107\end{array}$

$\begin{array}{ll}\text { A Details of components and devices } & 117\end{array}$

$\begin{array}{ll}\text { B Temperature gradient in hot-stage } & 121\end{array}$

$\begin{array}{ll}\text { C Velocities and velocity gradients in the cross-slot flow } & 125\end{array}$

$\begin{array}{ll}\text { D Material properties of the windows } & 129\end{array}$

$\begin{array}{ll}\text { Samenvatting } & 135\end{array}$

$\begin{array}{ll}\text { Acknowledgment } & 137\end{array}$

$\begin{array}{ll}\text { Curriculum Vitae } & 138\end{array}$ 


\section{Summary}

The resulting properties of a product made of semi-crystalline polymers strongly depends on both molecular properties and, via flow-induced structure formation, on the processing conditions applied. Consequently, the complete thermal-mechanical history has to be modeled in order to describe nucleation and crystallization kinetics and their dependence on flow.

Experimental results in the literature show that flow-induced crystallization correlates with the viscoelastic stresses, much more than with strain or strain rate, leading to the conclusion that chain orientation/extension is the governing phenomenon. The orientation of polymer chains can result in the development of anisotropic structures which influence the dimensional stability of the final product. A modeling tool is 
temperature calibration of the hot-stage, the DSC and the rheometer, a deviation in the rate of crystallization is found between these methods.

The modulus build-up in oscillatory shear as a function of space filling was compared for several isothermal crystallization experiments. No unique relation was found for both materials, apparently due to its extreme sensitivity for experimental errors, like the occurence of a trans-crystallization layer as observed in light microscopy.

Birefringence and Wide Angle X-ray experiments were performed in the cross-slot flow cell. The birefringence experiments showed the occurrence of 'streamlines' caused by the presence of the optical windows. This phenomenon is explained by the influence of shear gradients close to the windows. The WAXS experiment showed a highly oriented structure close to the outflow stagnation line, having orientations in the (110), (040) and (130) reflections. Orientation in both (110) and (130) corresponds to a highly oriented, non-branched structure. The size of the highly oriented structure was about $80[\mu \mathrm{m}]$ in inflow and at least $0.2[\mathrm{~mm}]$ in outflow direction.

The dominance of the stagnation point could not be observed in experiments in which flow was created above and crystallization was studied below the melting temperature. It is concluded that also the shear gradients have a large influence on the crystallization kinetics in this three-dimensional, complex flow.

Numerical simulations using the $S_{\mathrm{J} 2}$ model show that the influence of the shear gradients in the flow cell can be reduced by using a shorter flow time and/or using flow channels with a larger depth-to-height ratio. Moreover, the reproducibility of the thermal history in the flow cell needs to be improved, while the reproducibility of the deformation history was good. However, in order to perform short time deformation experiments, the set-up has to be changed to allow for larger torques. Finally, it was concluded that the strain hardening behavior in elongation for the Leonov model overestimates the first component of the Finger tensor, and therefore, the number of flowinduced nuclei for the $S_{\mathrm{J} 2}$ model. It is, therefore, recommended to use the Pompom model as a basis for a quantitative flow-induced crystallization model, that also can deal with complex flows. 


\section{Chapter 1}

\section{Introduction}

The resulting properties of a product made of semi-crystalline polymers strongly depends on both molecular properties and the processing conditions applied, i.e. the thermal-mechanical history experienced by the polymer in the process. Consequently, this thermal-mechanical history (e.g. in injection moulding, film blowing or fiber spinning) has to be modeled in order to describe nucleation and crystallization kinetics and their dependence on flow-induced structure formation. The ultimate goal is to develop a tool that is able to improve or even optimize polymer synthesis and industrial processes. However, many of the underlying relations are still not clear due to the complex mutual interaction of many parameters. Therefore, optimization of properties in industry is still done by expensive and time consuming trial and error methods, which -at best- are based on cumulative experience.

The need for such modeling tool became even more urgent since the development of novel metallocene-based catalysis. Compared to conventional Ziegler-Natta catalysis, metallocene-based polymers are much more well defined and possess a narrow molecular weight distribution. Moreover, differences in regularity of the polymer chains exist caused by sterical restrictions during polymer synthesis (Gahleitner et al. [45]).

The relation between molecular properties, processing conditions and final properties that results in the micro-structure formed, can be determined once recent developments in detailed modeling are condensed into constitutive equations that can be the input of continuum mechanical modeling. The relation between molecular weight distribution and linear viscoelastic properties (Mead [98], Pattamaprom et al. [110]) and that between rheology and flow-induced nucleation (Eder and JaneschitzKriegl [35], Zuidema [156]) has now become much more clear. Polymer rheology can at present quantitatively be described with the (extended) Pompom model (Inkson et al. [62], McLeish and Larson [97], Verbeeten et al. [143]). Consequently, a powerful tool could be obtained once these methods are combined to model the whole life cycle of a polymer: from the synthesis to properties via processing. However, much more advanced experimental methods and set-ups than used at present have to be developed to be able to validate the modeling and to obtain the necessary input parameters.

The objective of this study is to contribute to this last issue by developing and analyzing an extensional flow device for polymer melts and to measure and predict the resulting micro-structural developments and distributions. A cross-slot flow cell was 


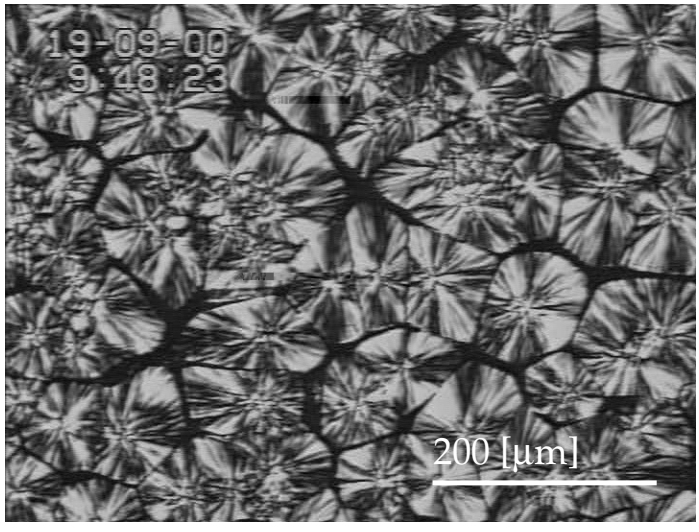

(a)

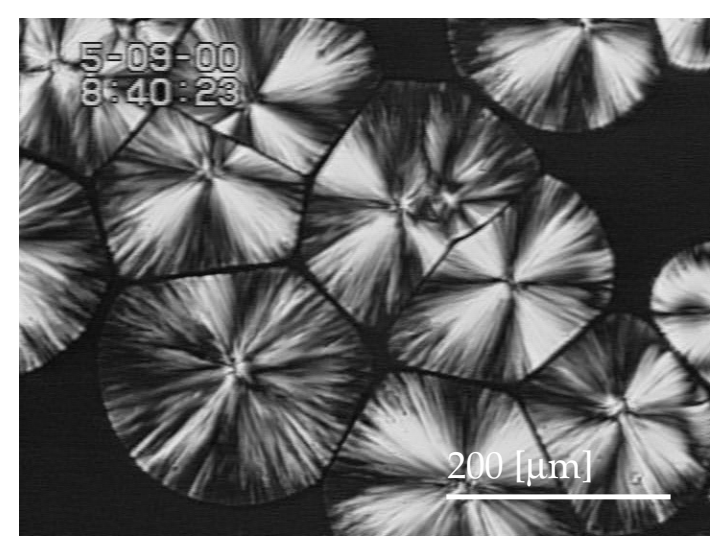

(b)

Figure 1.1: Quiescent crystallization of DSM StamylanP $13 \mathrm{E} 10(\mathbf{a})$ at $140\left[{ }^{\circ} \mathrm{C}\right]$ and (b) at $152\left[{ }^{\circ} \mathrm{C}\right]$.

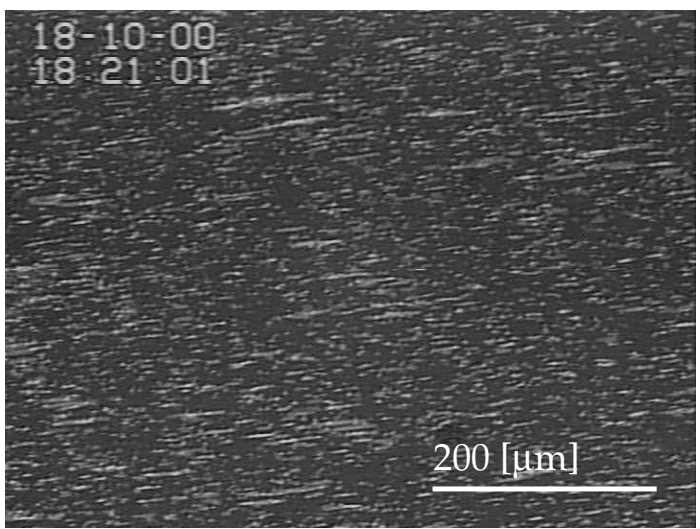

(a)

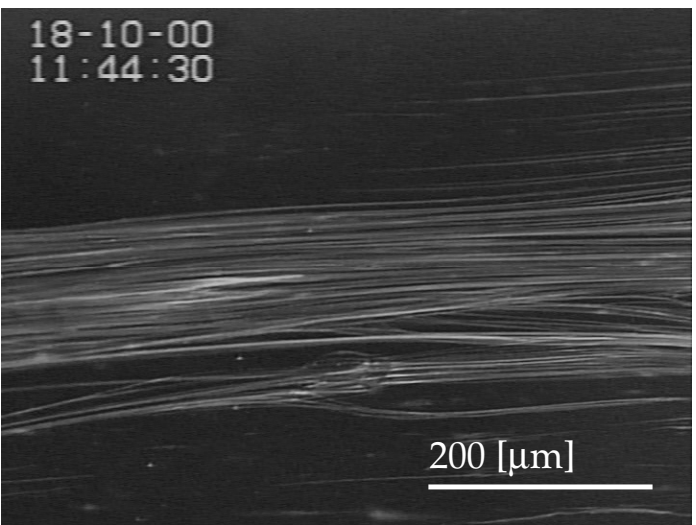

(b)

Figure 1.2: Crystallization after shear flow of DSM StamylanP $13 \mathrm{E} 10$ at 140 [ $\left.{ }^{\circ} \mathrm{C}\right]$. (a) Low shear rate. (b) High shear rate.

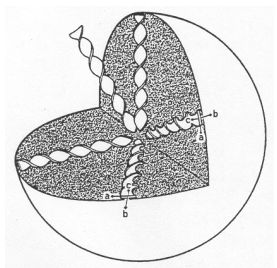

(a)

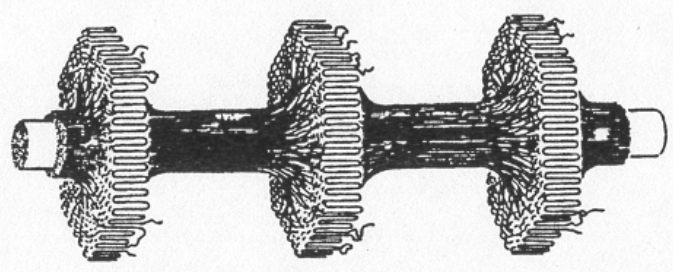

(b)

Figure 1.3: Schematic drawings of (a) a spherulite and (b) a shish-kebab structure (both taken from Keller and Kolnaar [72]). 
designed to be operated in the in-situ X-ray facilities at the European Synchrotron Radiation Facility (ESRF) in Grenoble, France, and a numerical tool was developed to predict the resulting structure in this cell.

\subsection{Crystallization of polymers}

\section{Observations}

Crystallization of polymers, not only the rate of crystallization but also the resulting partly- anisotropic structure, depends strongly on the thermal and deformation history of the material experienced during processing. An illustrative example is shown in Figures 1.1 and 1.2, obtained in a hot-stage with a microscope and two crossed polarizers using an isotactic Polypropylene (DSM, StamylanP 13E10). The influence of the crystallization temperature, under quiescent conditions and applying the same cooling rate, is shown in Figure 1.1. Birefringent spherulites consisting of outward rotating lamellae are found (Figure 1.3(a)), and the number of spherulites is much smaller at higher crystallization temperatures. This dependence on temperature is usually described by a linear exponential function for the number of nuclei. The influence of the thermal history is, moreover, reflected in the growth rate of spherulites, which is usually described by a quadratic exponential function between glass transition and melting temperature.

Deformation has an important influence on the orientation of the polymer chains and on the resulting anisotropy of the crystals formed, see Figure 1.2, where the same thermal history as in Figure 1.1 was applied. Flow was created by moving the upper glass plate of the sample for a few seconds at $140\left[{ }^{\circ} \mathrm{C}\right]$. A huge increase in the number of nuclei can be observed (Figure 1.2(a)) and if the deformation applied is strong enough, highly oriented structures can be observed (Figure 1.2(b)). Keller and Kolnaar [72] showed that these fiber structures can be created, beyond a certain elongational rate, by the high end tail of the molecular weight distribution that has the largest relaxation time. The lower molecular weight parts relax fast during and after deformation and crystallize later as lamellae nucleated by the fibers. The resulting shish-kebab morphology is shown in Figure 1.3(b). Eder and Janeschitz-Kriegl [35] showed that these structures are not only observed in (strong, irrotational) elongational flows, but can also be found in shear flows in a slit, close to the wall where viscoelastic stresses (and thus molecular orientation) can be very large. Moreover, shish-kebab structures were found in many polymer materials (e.g. PE [72], iPP [35], PA6 [123], PB [120, 122]). These anisotropic shish-kebab structures have an important influence on properties, like warpage and anisotropic shrinkage.

According to Keller and Kolnaar [72] chain extension promotes crystallization for two reasons. First, it increases the melting, and thus crystallization, temperature of the material, due to the lower entropy, and thus higher free energy, of an extended chain compared to a random coil. Crystallization is enhanced, since the super-cooling for any temperature below the melting temperature is increased. For example, the melting temperature of the oriented structure in Figure 1.2(b) was about 20 degrees higher than the melting temperature of the lamellae. Second, the extended chain is closer to its final configuration state in a crystal and has, therefore, a lower kinetic barrier to overcome 
than a chain in the random state.

Full chain extension can most easily be achieved in stagnation flows. An overview with references of the different geometries to create such flow is given by Schoonen [128] and Swartjes [136]. Examples, shown in Figure 1.4, include the two roll mill (Frank and Mackley [40], Geffroy and Leal [51]), the four roll mill (Crowley et al. [26], Dunlap and Leal [32], McHugh et al. [96]), the (lubricated) converging flow (with a free stagnation point; Aken and Janeschitz-Kriegl [1], Macosko et al. [89]), the cross-slot device (Gardner et al. [50], Miles and Keller [100]) and the opposed jets (JaneschitzKriegl et al. [63], Keller et al. [73], Mackay et al. [86]). The basic principle of a stagnation flow is, in all cases, the same: two liquid streams are impinged. A free stagnation point is created in the center where the extensional strain can become very high.

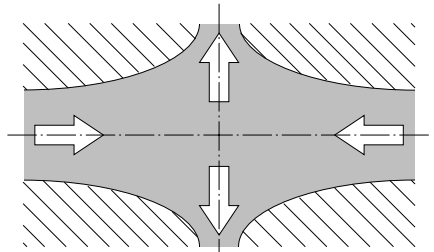

(a)

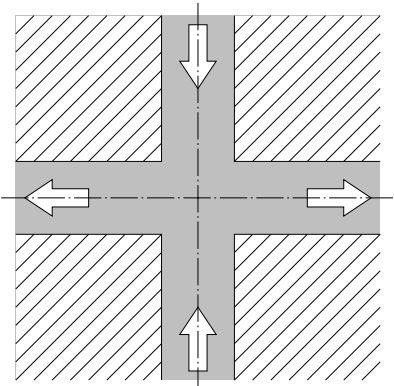

(b)

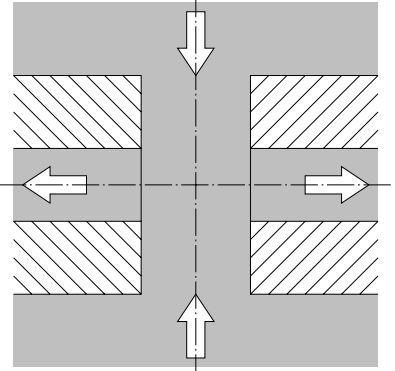

(c)

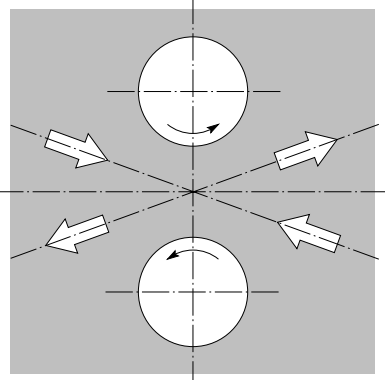

(d)

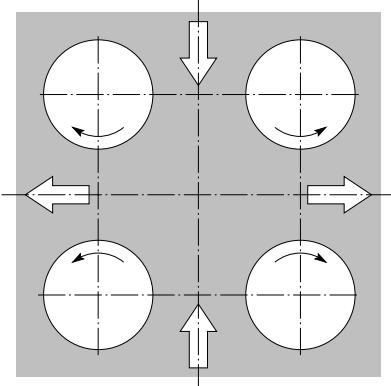

(e)

Figure 1.4: Devices to create stagnation flows for polymer liquids; (a) Lubricated converging flow [1, 89]. (b) Cross-slot device [50, 100]. (c) Opposed jets [63, 73, 86]. (d) Two roll mill $[40,51]$. (e) Four roll mill $[26,32,96]$.

In his review on flow-induced crystallization of solutions, McHugh [95] predicted that the influence on nucleation of an extensional gradient is much larger than that of a shear gradient. He calculated a transition from chain folded nucleation to fibrous nucleation at an elongational strain of about 20 percent of the chain contour length.

Tas [138] observed that in the film blowing process, mechanical properties of different LDPE's correlate extremely well with the viscoelastic stress at crystallization, much more than with strain or strain rate, leading to the conclusion that chain orientation/extension is the governing phenemenon. In this study a cross-slot flow cell will be used to investigate stress-induced crystallization in melts (of isotactic Polypropylene). This geometry was also used in the past by Keller and coworkers (Gardner et al. [50], Miles and Keller [100]) to investigate the interplay between flow and polymer conformation in an elongational flow for polymer solutions. 


\section{Definitions}

Two processes can be observed during crystallization: primary and secondary crystallization (Elias [37]). Primary crystallization can be considered as the growth of crystalline super structures, while secondary crystallization is the perfectioning in these structures (e.g. lamellar thickening). Secondary crystallization can be neglected in fast cooling processes (provided that crystallization at room temperature is slow or impossible), since the rate of primary crystallization is much higher than the rate of secondary crystallization.

Primary crystallization can on its turn be caused by two processes: primary and secondary nucleation. In primary nucleation, we can distinguish homogeneous and heterogeneous nucleation. Homogeneous nucleation is the formation of nuclei in the material itself, caused by thermodynamical reasons. Heterogeneous nucleation is nucleation at the surface of a substrate. This process is often the most important nucleation process. A more common expression for secondary nucleation is growth.

Gahleitner and Wolfschwenger [47] distinguished in heterogeneous nucleation three different processes: spontaneous, self- and external nucleation. Spontaneous nucleation is caused by heterogeneities in the material (dust, catalyst residues, degradation products or non-molten additives). Self nucleation occurs on not-completely molten parts of the polymer. External nucleation is caused by the addition of nucleating agents to increase crystallinity or to optimize properties (e.g. via a finer crystalline structure).

Elias [37] defined simultaneous nucleation and sporadic nucleation. In simultaneous nucleation, nuclei are created at the same time, while in sporadic nucleation the number of nuclei increases in time. The formation of flow-induced nuclei is an example of the creation of sporadic nuclei.

\subsection{Outline of the thesis}

We will investigate the influence of an extensional flow on the crystallization behavior of isotactic Polypropylene. Several experimental methods were used and a literature research was performed, see Chapter 2. Constitutive models that describe the rheological behavior and the coupling between rheology, nucleation and subsequent crystallization are presented in Chapter 3. Results on standard characterization techniques determining the rheological parameters and crystallization kinetics parameters for an accurate description of material behavior during quiescent crystallization and as a consequence of flow, are given in Chapter 4 .

A cross-slot device was used for in-situ X-ray and birefringence experiments. The behavior of the polymer in this cell is studied using a numerical model, i.e. a threedimensional spectral element method, in Chapter 5. Deformation history is determined by using particle tracking. Resulting stresses are calculated separately by using the constitutive equations. Experimental results obtained in the cross-slot flow cell are discussed in Chapter 6 and, finally, conclusions are drawn and recommendations for future research are given in Chapter 7. 



\section{Chapter 2}

\section{Methods and materials}

Backgrounds and principles of standard experimental methods used in this study are summarized in Section 2.1. The choice of the polymer used and its characteristics, especially in relation with the experimental methods, are discussed in Section 2.2. Finally, a short review of experiments on flow-induced nucleation and crystallization is given in Section 2.3.

\subsection{Experimental methods}

Several methods are known to obtain information on crystallization. Some basic principles of the standard methods used are explained in this section. Table 2.1 summarizes the methods, the structure measures that can be obtained, and the possibility to measure orientation. All methods give information on the onset and rate of crystallization. Absolute crystallinity can be obtained, indirectly, by DSC and WAXS. The integrated intensity in SAXS is linearly proportional to the crystallinity in the early stages of crystallization (Wang et al. [148]). DSC is not able to give a direct measure for the amount of oriented material.

\begin{tabular}{|l||l|c||c|}
\hline Method & Name & Symbol & Orientation \\
\hline \hline Differential Scanning Calorimetry (DSC) & Crystallinity & $\chi$ & \\
\hline Microscopy & Space filling & $\xi_{\mathrm{g}}$ & $\mathrm{x}$ \\
\hline Wide Angle X-ray Scattering (WAXS) & Crystallinity & $\chi$ & $\mathrm{x}$ \\
\hline Small Angle X-ray Scattering (SAXS) & Crystallinity & $\chi$ & $\mathrm{x}$ \\
\hline Birefringence & Birefringence & $\Delta \mathrm{n}$ & $\mathrm{x}$ \\
\hline Rheometry & Modulus & $\mathrm{G}^{\prime}, \mathrm{G}^{\prime \prime}$ & $\mathrm{x}$ \\
\hline
\end{tabular}

Table 2.1: Methods used with crystallinity-measures and possibility to measure orientation.

\subsubsection{DSC}

Differential Scanning Calorimetry (DSC) is an accurate, although limited (Eder and Janeschitz-Kriegl [35]) method to investigate the melting and crystallization behavior 
of materials. In this device the temperature difference of two pans, one with and one without a sample, is kept constant using a bridge circuit. The power needed to keep this circuit in balance is measured. The crystallization enthalpy is calculated by integrating the heating power of the crystallization peak in time, divided by the sample mass. Comparison with the theoretical enthalpy value of $100 \%$ crystalline material and assuming a linear relation between released heat and crystallinity, gives a value for the absolute crystallinity. Crystallization onset, speed, and absolute crystallinity can be determined for isothermal crystallization conditions. It is important to notice that an additional method is needed to obtain some morphological information. More information on thermo-analytical methods can be found in Gallagher [49].

\subsubsection{Rheometry}

A crystallizing polymer with growing superstructures, spherulites and/or shish-kebab structures can be regarded as a dispersion. Dispersion rheology gives relations between rheological properties and the amount of filler (in this case space filling). The influence of space filling on viscosity and modulus will be discussed below. Basics of dispersion rheology can be found in Barnes et al. [8], while a review on the melt rheology of filled thermo-plastics is given in Khan and Prud'Homme [74].

Viscosity tends to go to infinity in dispersions at the maximum packing fraction $\left(\phi_{\max }\right)$. This value depends on the arrangement of the particles and ranges from 0.52 (cubic) to 0.74 (face centered cubic and hexagonal) for monodispers systems. The maximum packing fraction increases with broader particle size distributions and decreases with increasing anisotropic shape (Barnes et al. [8]).

For concentrated Newtonian suspensions, the Krieger-Dougherty relation describes the relation between viscosity and volume fraction for hard spheres:

$$
\frac{\eta(\phi)}{\eta(0)}=\left(1-\frac{\phi}{\phi_{\max }}\right)^{-[\eta] \phi_{\max }}
$$

with the intrinsic viscosity [ $\eta]$. For dilute suspensions, for which hydrodynamic interactions between the spheres can be neglected, this equation equals the one proposed by Einstein (in which $[\eta]=5 / 2$ ). Experimental fits on the Krieger-Dougherty relation have shown that the product of the intrinsic viscosity and the maximum packing fraction is equal to 1.92 for sub-micron spheres, 2.0 for $40[\mu \mathrm{m}]$ spheres, and 2.48 for glass rods having a size of $30 \times 700[\mu \mathrm{m}]$ (Barnes et al. [8]). The Krieger-Dougherty relation for these cases are shown in Figure 2.1. The shape of the viscosity versus volume fraction for spherical particles is not dependent on particle size.

Four interesting, experimental phenomena of filled systems in (oscillatory, transient and steady) shear and (transient) extensional flows were described by Khan and Prud'Homme [74]. First, a yield stress is observed in filled systems. For increasing filler fraction, $\eta$ becomes unbounded at low frequensies with slope -1 and $G^{\prime}$ becomes constant. Second, the dynamic response becomes more strain dependent than that of a pure melt. Consequently, the amplitude of the strain should be small enough to be in the linear viscoelastic behavior of filled systems. Third, particle filled systems have reduced elasticity. The ratio of the normal stress difference over the shear stress 


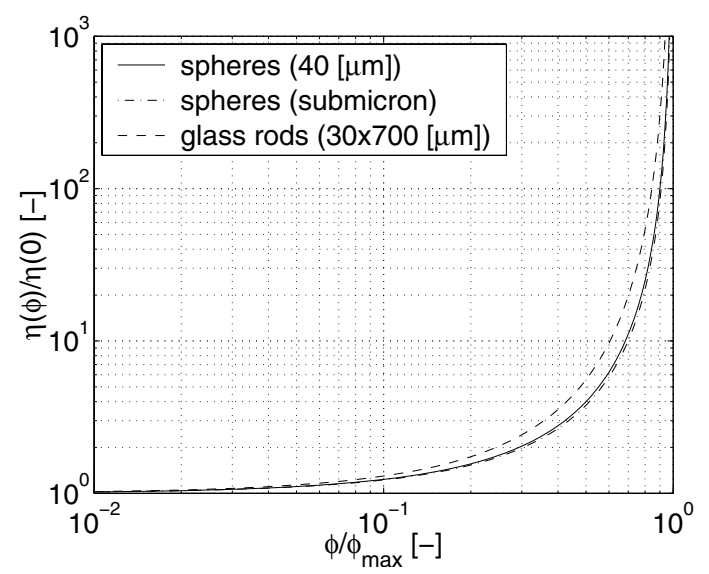

Figure 2.1: Krieger-Dougherty relation for three different particles (Barnes et al. [8]).

decreases for particle filled systems and increases for fiber filled systems. Fourth, transient properties of a filled system are not significantly different from that of a pure melt.

Khanna [75] related the transformed fraction $\phi$ with a scaled modulus $G^{\prime}$ :

$$
\phi=\frac{G^{\prime}(t)-G^{\prime}(0)}{G^{\prime}(\infty)-G^{\prime}(0)}
$$

with the time in between brackets. Modulus $\mathrm{G}^{\prime}(0)$ is the initial modulus and modulus $G^{\prime}(\infty)$ is the plateau value reached at the end of crystallization. Khanna used this equation for PA6 and iPP. Boutahar et al. [19] investigated the difference in the influence of crystallization on the rheological behavior for iPP and PE. According to these authors, iPP behaves as a suspension and PE as a colloid during crystallization. The filler fraction was assigned to spherulites for iPP and crystallites for PE. A yield effect was observed from the beginning of the crystallization for PE $(\phi=0.084[-])$ and was delayed for iPP $(\phi=0.4[-])$. The relation proposed by Khanna could be used for iPP, where the transformed fraction equals the volume fraction, but not for PE, where it equals the volume of crystallites inside the spherulites. Consequently, a unique relation that holds for crystallizing materials is difficult to obtain.

In conclusion, oscillatory experiments in a simple shear rheometer can be used to monitor crystallization. It is important to use small strains, because the dynamic response is very sensitive to the strain in filled systems.

\subsubsection{Birefringence}

Birefringence, or double refraction, is caused by an optical anisotropy in a material (Born and Wolf [17], Hecht and Zajac [58]). The optical axis in birefringent, polymer materials is defined in the direction of the chain axis (the c-axis in the crystal unit cell).

Three different interactions of a birefringent medium and a light wave can be distinguished, depending on the relative orientation of the optical axis and the propagation direction of the wave: 
- The optical axis is parallel to the propagation direction. The two orthogonal field components will experience the same retardance.

- The optical axis is perpendicular to the propagation direction. The two orthogonal field components will experience a different retardance and will coincide in space.

- All the other cases. The two orthogonal field components will experience a different retardance and will not coincide in space.

In the last two cases, the two orthogonal waves will travel with a different velocity through the medium, due to the difference in refraction . On exit of the birefringent medium, the two waves have different phases. This phase difference $\delta$ is related to the principle refractive index difference or birefringence $\Delta \mathrm{n}$, according to

$$
\delta=\frac{2 \pi d}{\lambda} \Delta n
$$

with $\mathrm{d}$ the thickness of the birefringent material and $\lambda$ the wavelength.

The principles of birefringence are used in optical microscopy, and in flow-induced birefringence and crystallization studies.

\section{Polymer crystals}

Polymer crystals can cause birefringence depending on the crystal unit cell. A crystal can be optically isotropic $\left(\mathrm{n}_{1}=\mathrm{n}_{2}=\mathrm{n}_{3}\right)$, uniaxial $\left(\mathrm{n}_{1}=\mathrm{n}_{2}<\mathrm{n}_{3}\right)$ or biaxial $\left(\mathrm{n}_{1}<\mathrm{n}_{2}<\mathrm{n}_{3}\right)$. An isotropic crystal behaves optically like an amorphous material. The uniaxial and biaxial crystals are birefringent. The refractive indices $\mathrm{n}_{1}, \mathrm{n}_{2}$ and $\mathrm{n}_{3}$ correspond to the a-, b- and c-axis of the crystal. Cubic crystal systems are optically anisotropic; trigonal, tetragonal and hexagonal crystal systems are uniaxial, and triclinic, monoclinic and orthorhombic crystal systems are biaxial. More background information about the optics of crystals can be found in Born and Wolf [17].

Sign and magnitude of the birefringence of superstructures can be determined in optical microscopy. The birefringence of spherulites and oriented structures depends on the refractive indices of the crystals and their arrangements. The birefringence of those structures is defined by the difference of the radial and tangential refractive index:

$$
\Delta=n_{r}-n_{t}
$$

Detailed information on the optical properties of spherulites is discussed in Haudin [57].

\section{Flow-induced birefringence}

Polymer chains subjected to flow will orient in flow direction and will cause flowinduced birefringence. It originates from the difference of polarizability of a polymer chain along its backbone and perpendicular to that. A flowing polymer can be regarded as a uniaxial $\left(\mathrm{n}_{1}=\mathrm{n}_{2}<\mathrm{n}_{3}\right)$ birefringent material. The principle of flow-induced 
birefringence was used in rheology to test constitutive equations by relating the birefringence with stresses using the Stress Optical Rule (SOR; e.g. Macosko [88], Schoonen [128]).

Flow geometries were used that can be considered as two-dimensional in which a minimal channel depth-to-height ratio of eight was used. Refraction index tensor and stress tensor were related directly via the stress optical coefficient. For smaller ratios, the varying refraction index in the depth of the channel had to be taken into account. A method was described in Schoonen [128, 129] and Swartjes [136], where varying optical properties and stresses in depth were integrated and compared for the flow of a polyisobutylene solution in a cross-slot flow cell.

\section{Flow-induced crystallization}

A combination of the birefringence caused by the (flowing) amorphous and the crystalline phase can be observed in flow-induced crystallization experiments (Bushman and McHugh [23], White and Cakmak [153]). The flow-induced birefringence will disappear after relaxation of the amorphous part. Differences in refractive indices of the amorphous and crystalline phases can give an extra contribution to the birefringence and is called form birefringence (Born and Wolf [17], White and Cakmak [153]).

The onset and growth of oriented, crystalline structures can be determined in birefringence measurements (Eder and Janeschitz-Kriegl [35], Kumaraswamy et al. [76], Liedauer et al. [82]). Three measures are obtained, namely the turbidity, and the transmitted intensity through parallel, and crossed polarizers. The increase in turbidity during crystallization is caused by the scattering of growing crystalline structures. Therefore, the intensity through crossed polarizers has to be normalized by the initial intensity of the beam (e.g. Eder et al. [36, Figure 36]). Depolarization due to the scattering is negligible during the initial stages of crystallization. If the total intensity approaches zero and depolarization influences the measurement, the relation between birefringence and measured intensity is not valid anymore (Kumaraswamy et al. [76]).

The time that the retardation equals half times the wavelength is a characteristic measure for the growth of oriented structures (Eder and Janeschitz-Kriegl [35], Liedauer et al. [82]). This time corresponds to the maximum that can be observed in the normalized transmitted intensity through crossed polarizers. Eder and Liedauer could determine the average distance between oriented threads by using this characteristic time and corresponding birefringence.

Kumaraswamy et al. [76] showed three correlations between oriented structures and birefringence measures. First, the time for the transmitted intensity to decrease to half its value did not decrease further with increasing shear time. Second, the transient intensity transmitted through crossed polarizers showed an upturn during shear, which did not relax after cessation of flow. Third, a maximum in the birefringence could be observed after flow as discussed above.

In conclusion, a clear relation between birefringence and oriented structures at the initial stage of crystallization was shown in literature. The average distance between cylindrical structures could be determined. However, the relation between birefringence and space filling or crystalline fraction is difficult to obtain, especially when both oriented as spherulitical structures are obtained along the path of the light beam. 


\subsubsection{Microscopy}

Optical microscopy is widely used to investigate crystallization in quiescent conditions and as a consequence of flow (e.g. Duplay et al. [33], Jay et al. [64], Eder and JaneschitzKriegl [35]). In between crossed polarizers two typical observations were made for quiescent crystallization (Haudin [57]), namely the Maltese cross and the phenomenon of ringed spherulites.

These phenomena can be explained by using Müller calculus. Transmitted light for a birefringent material oriented at angle $\chi$ in between crossed polarizers, is given by

$$
I_{1}=P_{90} \cdot M_{\delta}(\chi) \cdot P_{0} \cdot I_{0}
$$

Using the Müller matrices for the polarizers (Hecht and Zajac [58, Chapter 8]) and the birefringent material ( $\mathrm{Li}$ and Burghardt [80]), the following equation can be derived:

$$
I_{1}=\frac{1}{2} I_{0} \sin ^{2}(2 \chi) \cdot \sin ^{2}\left(\frac{1}{2} \delta\right)
$$

Equation (2.6) shows that extinction occurs if

1. $\chi=0$ or $\pi / 2$ for the position in the spherulite parallel or perpendicular to the analyzer and polarizer. This results in the Maltese cross.

2. $\delta=0$. This occurs for uniaxial crystals at positions where $n_{r}=n_{t}$ as a consequence of the outward rotating lamellae in a spherulite. This results in ringed spherulites.

These observations will be most clear in two-dimensional samples (thin samples and relatively large spherulites).

White light introduces an interference phenomenon, caused by the dependence of the phase difference on the wavelength (Equation (2.3)). For a certain retardation, it is possible that one color is linear polarized along the analyzer axis, one color is linear polarized along the generator axis, and the other components are elliptically polarized. As a consequence, interference colors can be seen. The two color components that are most affected are called complementary. Examples of such complementary colors are blue/yellow and red/green (Hecht and Zajac [58]). In conclusion, colors within spherulites are directly related to local variations in retardation, which affects the polarization state of the polychromatic wave.

The sign of birefringence can be obtained by using an additional wave-plate (Haudin [57]). The visible spectrum is shifted, by introducing an extra path difference. Depending on the color, the sign of birefringence can be determined.

In this study, microscopy will be used to investigate final morphologies and to determine nucleation and growth under quiescent conditions.

\subsubsection{X-ray scattering}

Scattering of an electro-magnetic wave by an electron can occur in an inelastic or elastic way. Inelastic, or Compton scattering occurs when a part of the energy of a photon is transferred to the electron. The wavelength is increased due to the decreased energy of the photon. Moreover, no relation can be defined between the phase before and 
after the collision. Elastic, or coherent scattering occurs when none of the energy of the photon is transferred to the electron. The photon changes direction after a perfect collision with the electron. The scattered beam has the same wavelength and frequency as the incident beam.

Wide Angle X-ray Scattering (WAXS) and Small Angle X-ray Scattering (SAXS) are examples of coherent scattering methods. The measurement ranges of these two methods are given in Table 2.2. Crystallites are recovered in the wide, while lamellae are recovered in the small angle region. In the next part, these two methods will be discussed in more detail. Information on WAXS and SAXS can be found in Cullity [27] and Glatter and Kratky [52].

\begin{tabular}{|l||c|c|l|}
\hline Method & $2 \theta_{\mathrm{Cu}}$ range [-] & d-range $[\mathrm{nm}]$ & Remark \\
\hline \hline WAXS & $3 \leq 2 \theta \leq 50$ & $2.94 \geq \mathrm{d} \geq 0.18$ & crystallites \\
\hline SAXS & $0.022 \leq 2 \theta \leq 3$ & $400 \geq \mathrm{d} \geq 2.94$ & lamellae \\
\hline
\end{tabular}

Table 2.2: Measuring range of WAXS and SAXS ( $2 \theta_{\mathrm{Cu}}$ in degrees; $\left.\lambda=0.154[\mathrm{~nm}]\right)$.

\section{Wide Angle X-ray Scattering}

Scattering by a lattice in a crystal is influenced by all the atoms in that lattice and interference of the scattering can occur. Scattering by an atom is only determined by its electrons. The nucleus does not influence the scattering, because its mass is much larger than an electron mass. The condition for constructive interference can be explained by using Figure 2.2(a). The two waves will be in phase if the path difference

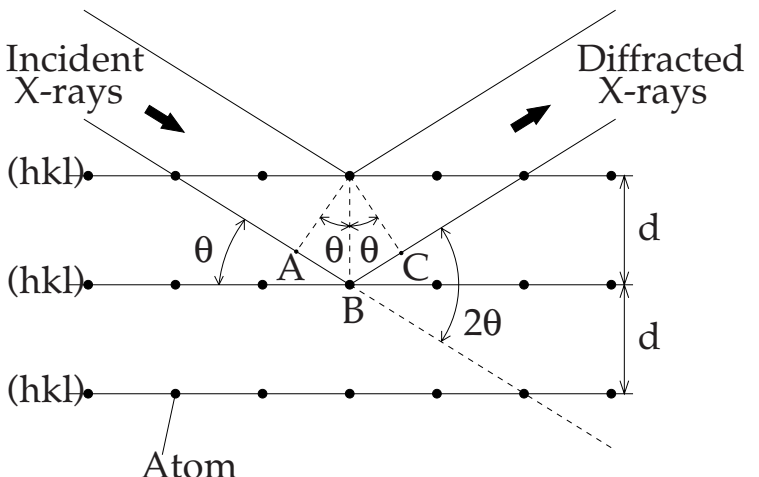

(a)

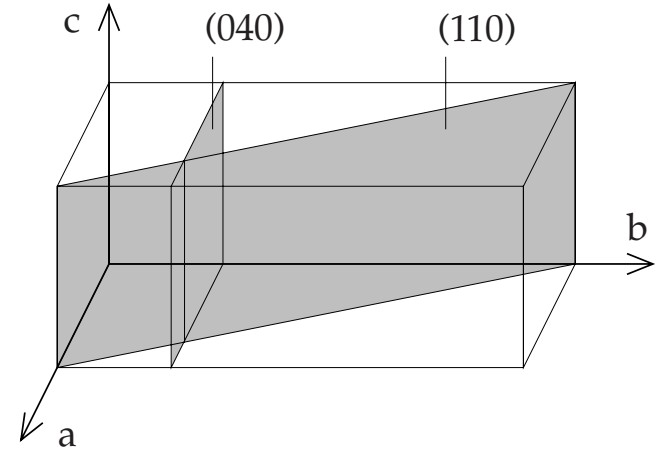

(b)

Figure 2.2: (a) Diffraction from two subsequent (hkl) planes in a crystal (b) Monoclinic unit cell of iPP with (110) and (040) plane.

$\mathrm{AB}+\mathrm{BC}$ (Figure 2.2) is equal to a whole number of $\mathrm{n}$ wavelengths. This condition is described by Bragg's law:

$$
n \lambda=2 d \sin \theta
$$

The inverse relation between the distance and the scattering angle as observed in Table 2.2 is clear in this equation. Bragg's law states that interference will (only) occur 
when all scattered waves from planes in the crystal are in phase. Normally, a small range of scattered intensities around the Bragg's angle will be observed. The limits of this range are captured by destructive interference caused by the first and and last plane in the crystal. Consequently, a relation can be derived between crystal size and width of the scattered peak (i.e. the Scherrer equation). Destructive interference will occur more easily in large, perfect crystals than in small crystals due to the larger amount of crystal planes. It can be concluded that the width of the diffraction peak increases as the crystal size decreases.

The diffraction planes are described with Miller indices (hkl), which are defined as the reciprocals of the intercepts the plane makes with the crystallographic axes. A plane with Miller indices ( $\mathrm{hkl}$ ) intersects the axes $\mathrm{a}, \mathrm{b}$ and $\mathrm{c}$ at $\mathrm{a} / \mathrm{h}, \mathrm{b} / \mathrm{k}$ and $\mathrm{c} / \mathrm{l}$ respectively. For example, the definition of the (110) and (040) planes in a monoclinic cell of iPP are given in Figure 2.2(b).

Braggs law shows that the scattering angle depends on the lattice distance. So, scattered angles can be used to recognize specific materials. The intensity of the diffracted beam is proportional to the square of the absolute value of the structure factor $\mathrm{F}$. This factor sums the waves scattered by each atom, described by a complex exponential function:

$$
F_{h k l}=\sum_{n=1}^{N} f_{n} e^{2 \pi i\left(h u_{n}+k v_{n}+l w_{n}\right)}
$$

with atomic scattering factor $\mathrm{f}$ and atom coordinates $(\mathrm{u}, \mathrm{v}, \mathrm{w})$. Equation (2.8) shows that the structure factor is independent of the shape and size of the unit cell. The absolute value of $\mathrm{F}$ describes the ratio between the amplitude of a wave scattered by all atoms of a unit cell and one electron. The atomic scattering factor describes the ratio between the amplitude of a wave scattered by an atom and one electron. It describes the efficiency of the scattering by an atom and is equal to the atomic number $Z$, which gives the number of electrons, in the forward scattering direction (in-phase scattering of all electrons) and decreases for increasing $\sin (\theta) / \lambda$. So, the intensity of the diffracted beam depends on the amount of atoms in the lattice plane and on the scattering angle.

\section{Small Angle X-ray Scattering}

Two waves, which are coherently scattered by a particle, are more or less in phase at small angles. Consequently, intensity does not depend on the scattering angle. The scattered function becomes more narrow for larger particles. In case of ordering of regions with different electron densities, interference will also occur and a maximum at small angles can be seen.

The position of the scattering peak in SAXS for semi-crystalline polymers is related to the long period, which is related to the distance between lamellae (Figure 2.3). It can be estimated by applying Bragg's law on the peak position of the SAXS data

$$
L=\frac{2 \pi}{q}
$$




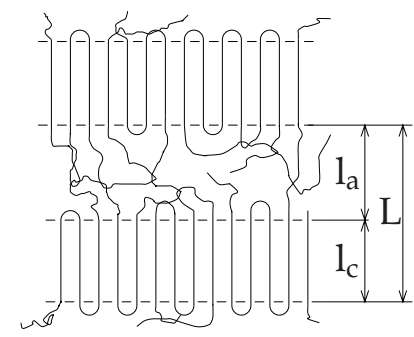

Figure 2.3: Schematic drawing of two lamellae. L: long distance; $1_{c}$ : crystalline lamellar thickness; $l_{\mathrm{a}}$ : amorphous lamellar thickness.

with the scattering vector $\mathrm{q}$, which is defined as

$$
q=\frac{4 \pi \sin (\theta)}{\lambda}
$$

The behavior of the long period as function of temperature and time is described in Verma and Hsiao [146]. The long period increases with crystallization temperature and decreases with time. One of the feasible explanations of the decreasing long period (shifting of the peak maximum to larger scattering angles) in time is the insertion of new lamellae during primary crystallization.

\subsection{Materials selection: iPP}

Important semi-crystalline materials are polyethylene, polypropylene (polyolefins), polyamides and poly-(ethylene terephthalate) (a thermoplastic polyester). From a practical point of view, polyethylene is the most difficult polymer to study in crystallization, because the maximum number of nuclei and growth rate are very high (Table 2.3). Therefore, for PE (high) cooling rates, necessary to obtain isothermal crystallization conditions are for most experimental set-ups not realistic (Eder [34]). Polypropylene demands a much lower cooling rate and offers an interesting crystallization temperature range from $\mathrm{T}_{\mathrm{c}}=125\left[{ }^{\circ} \mathrm{C}\right]$, where maximum crystallinity is obtained in minutes, to $\mathrm{T}_{\mathrm{C}}=150\left[{ }^{\circ} \mathrm{C}\right]$, where this takes a day. For this reason and for its importance for industry and its known characteristics, as described in literature, this material was chosen in this study.

\begin{tabular}{|l||r|r|r|}
\hline Material & $\mathrm{G}_{\max }[\mathrm{m} / \mathrm{s}]$ & $\mathrm{N}_{\max }\left[\mathrm{m}^{-3}\right]$ & $\mathrm{q}_{\text {crit }}[\mathrm{K} / \mathrm{s}]$ \\
\hline \hline HDPE & $2 \cdot 10^{-5}$ & $\sim 10^{17}$ & 93 \\
\hline iPP & $5 \cdot 10^{-6}$ & $\sim 10^{14}$ & 9.4 \\
\hline PET $\left(\right.$ low $\mathrm{M}_{\mathrm{w}}$ ) & $1.2 \cdot 10^{-7}$ & $\sim 10^{15}$ & 0.19 \\
\hline PET $\left(\right.$ high $\mathrm{M}_{\mathrm{w}}$ ) & $2 \cdot 10^{-8}$ & $\sim 10^{15}$ & 0.03 \\
\hline
\end{tabular}

Table 2.3: Material properties of some polyolefins and polyesters. G: growth rate; N: number of nuclei; $\mathrm{q}_{\text {crit }}$ cooling rate necessary to obtain less than one percent crystallinity after reaching the isothermal crystallization temperature. Data were taken from Eder [34]. 
Polymorphism of isotactic polypropylene has attracted scientific interest already for more than forty years (Brückner et al. [22]). Isotactic polypropylene can crystallize in three different unit cell modifications: the $\alpha, \beta$ and $\gamma$ phase. Moreover, it can be observed in a mesomorphic phase having a degree of order intermediate between the crystalline and amorphous phase. All the phases show a three-fold helix $\left(3_{1}\right)$ of the same length of $6.5[\AA ̊]$. An elucidation of iPP was given by Brückner et al. [22] and a review and molecular analysis was given by Lotz et al. [84]. Structure and morphology of iPP is described in Karger-Kocsis [70]. Transformation during recrystallization can be found in Varga [141].

The monoclinic $\alpha$ phase is the most stable crystalline form. Transformation of the other phases into $\alpha$ is obtained by recrystallization (annealing at a relatively high temperature below the melting temperature $T_{m}$ of $\alpha$ ).

The $\beta$ phase is formed once a sample has been subjected to large strain rates (e.g. Duplay et al. [33], Jay et al. [64], Monasse [101], Somani et al. [134], Varga and KargerKocsis [142], Wu et al. [154]) and/or under specific crystallization conditions such as high crystallization temperatures or large temperature gradients (Varga [141]). Meille et al. [99] proposed two possible models to describe this phase: a trigonal and a special orthorhombic lattice. Recently, Dorset et al. [28] described the $\beta$ phase with a model of 'frustrated' chain packing. Before these investigations, this phase was described by a hexagonal unit cell. Some uncertainties about its structure still remain. The $\beta$ phase cannot be obtained in a fiber structure, because it transforms into $\alpha$ upon stretching (Stocker et al. [135]). However, it was found during shear as unoriented crystallites (Somani et al. [134]) and after cessation of shear (Duplay et al. [33]). Varga and KargerKocsis [142] showed that the amount of $\beta$ was largest if melt-shearing was applied below $140\left[{ }^{\circ} \mathrm{C}\right]$. This amount decreased with increasing shear temperature, irrespective of subsequent crystallization below 140 [ ${ }^{\circ} \mathrm{C}$ ]. According to Varga and Karger-Kocsis [142] $\beta$ nuclei can be formed on $\alpha$ row nuclei, which explains the unoriented $\beta$ crystallites of Somani et al. [134]. The $\beta$ phase influences final product properties, i.e. its presence improves the impact strength and reduces the stiffness (Gahleitner and Wolfschwenger [47], Karger-Kocsis et al. [71]). The higher linear growth of the $\beta$ phase, in comparison with the $\alpha$ phase, below $140\left[{ }^{\circ} \mathrm{C}\right]$ was described by Varga [141].

The (face centered) orthorhombic (Brückner et al. [22]) $\gamma$ phase is formed as a consequence of special processing conditions, like high shear rates (Kalay et al. [69]) or high pressures, and is due to the special structure of the polymer. Disturbances of the chain, like stereo-irregularities, seem to favor this modification as discussed by Gahleitner et al. [45]. According to Kalay et al. [69] this phase is associated with pronounced molecular orientation.

The mesophase was observed in rapidly quenched samples (Brückner et al. [22]). Corradini et al. [25] showed the existence of local correlations between chains in this phase close to those in the monoclinic $\alpha$ phase.

An unique characteristic property of iPP is lamellar branching. It occurs within the $\alpha$ phase ( $\alpha-\alpha$ branching) or between the $\alpha$ and $\gamma$ phase ( $\alpha-\gamma$ branching). This feature was described on a molecular level by Lotz and Wittmann [83], and Lotz et al. [84]. In spherulites, mother and daughter lamellae are 80 or 100 degrees apart. This angle is equal to the monoclinic $\beta$ angle (Table 2.4). The growth direction of the iPP-lamellae is in the a-direction and chains are in c-direction (Norton and Keller [108]). In daughter 


\begin{tabular}{|l|l||r|r|r|}
\hline \multicolumn{2}{|c|}{} & $\alpha$ phase & $\beta$ phase & $\gamma$ phase \\
\hline \hline \multirow{3}{*}{ length [A] } & $\mathrm{a}$ & 6.66 & 11.01 & 8.54 \\
\cline { 2 - 5 } & $\mathrm{b}$ & 20.78 & 11.01 & 9.93 \\
\hline & $\mathrm{C}$ & 6.50 & 6.50 & 42.41 \\
\hline \multirow{3}{*}{ angle (degrees) } & $\alpha$ & 90.0 & 90.0 & 90.0 \\
\cline { 2 - 5 } & $\beta$ & 99.6 & 90.0 & 90.0 \\
\cline { 2 - 5 } & $\gamma$ & 90.0 & 90.0 & 90.0 \\
\hline
\end{tabular}

Table 2.4: Dimensions of the unit cell for the three phases (Brückner et al. [22], Lotz et al. [84]).

lamellae two new directions $\mathrm{a}^{*}$ and $\mathrm{c}^{*}$ can be defined, which refer to the new growth and chain directions. These new axis are obtained by rotating the original axis around the b-axis with 80 or 100 degrees. The b-direction is not affected by branching.

In the next section, some important observations specific for iPP of the experimental methods described will be discussed. (It will be denoted if a different material was used to illustrate specific observations and phenomena).

\section{DSC}

DSC can be used for the determination of the equilibrium melting temperature of iPP as described in Monasse and Haudin [103]. The melting point was determined for several samples which were crystallized at different temperatures, leading to different spherulite sizes. Next, the equilibrium melting temperature was determined by intersection of the melting and crystallization line. Using this approach, two different equilibrium melting temperatures were found: $187 \pm 2\left[{ }^{\circ} \mathrm{C}\right]$ for crystals formed at crystallization temperatures between 115 and $130\left[{ }^{\circ} \mathrm{C}\right]$ and $208 \pm 8\left[{ }^{\circ} \mathrm{C}\right]$ for higher crystallization temperatures. This difference is related to the different morphological features (i.e. cross hatching), which depends on the crystallization temperature (see Birefringence section). Samuels [124] extrapolated a value of $220\left[{ }^{\circ} \mathrm{C}\right]$ for fully oriented chains. When constrainted, iPP does not melt up to temperatures of 210 [ ${ }^{\circ} \mathrm{C}$ ] (Bastiaansen and Lemstra [9]). This is the basis of trying to make the so-called all-PP composites.

Alfonso and Ziabicki [2] considered the influence of annealing time and temperature for three kinds of polypropylenes in DSC experiments. Higher melt temperatures and longer melting times decrease the crystallization rate. This effect is attributed to a decreasing concentration of athermal nuclei which disappear after prolonger heating. However, the influence of time shows an asymptotic behavior, above which longer times don't affect the crystallization rate. This behavior is attributed to heterogeneous nuclei, which are insensitive to annealing time and temperature.

\section{Rheometry}

Oscillatory experiments were used to investigate quiescent crystallization (Boutahar et al. [18, 19], Pogodina and Winter [115]) and flow-induced crystallization (Pogodina et al. [116], Vleeshouwers and Meijer [147]). The results of these experiments will be discussed in more detail. 
Boutahar et al. [18] showed an increase in $\mathrm{G}^{\prime}$ as a function of space filling for several frequencies for an iPP crystallizing at $135\left[{ }^{\circ} \mathrm{C}\right]$. This increase was more pronounced at low frequencies. At high filler contents an unique plateau was reached, which was frequency independent. Two regions could be distinguished in time. First, the material behaved as a viscoelastic fluid up to a space filling of 0.4 [-]. For higher space filling values, the material behaved as a viscoelastic solid. Maximum packing fraction was in between 0.52 (cubic) and 0.68 (centered cubic). DSC data was enclosed by these two packing models.

Pogodina and Winter [115] described the quiescent crystallation behavior of four commercial polypropylenes in terms of a physical gelation process using a rheometer. The time to reach the gel point decreases exponentially with the supercooling, and the gel becomes stiffer with increasing supercooling. The absolute critical crystallinity at the gel point was found to be very small (about $2 \%$ or less). This seems to contradict with the findings of Boutahar et al. [18], where space filling and crystallinity followed the same curve. However, in Pogodina et al. [114], Pogodina and Winter [115] DSC data were extrapolated to the higher temperatures where the rheological measurements were performed. This could introduce an error in the comparison. The rheological gel time was very close to the gel time determined with small angle light scattering (SALS; Pogodina et al. [114]). The volume fraction was about 0.5 at the gel point. The influence of shear was studied in Pogodina et al. [116]. Shear creep was applied to the supercooled melt either during the nucleation stage or during crystallization, after 30 minutes at $\mathrm{T}_{\mathrm{c}}$. This last setting leads to a stronger decrease in gel time (faster crystallization) compared to strain applied at the nucleation stage.

Nagatake et al. [106] developed a shear flow thermal rheometer (SFTR): a standard shear controlled rheometer was equipped with differential thermal analysis. In this device a direct comparison between relative crystallinity and modulus was obtained. Crystallinity at the gelpoint seems to be much larger (at most 50\%) than the few percentage measured by Pogodina at the gelpoint. Nagatake performed both quiescent and shear experiments, but the plate-plate configuration used gives a non-uniform deformation history in the case of the shear experiments.

All references described above found a space filling $(\phi)$ of about 0.5 at the 'gelpoint', the point that structures start to impinge. However, results on the absolute crystallinity of those structures at the gelpoint were contradictory.

Vleeshouwers and Meijer [147] investigated the influence of molecular weight (distribution) for three isotactic polypropylenes. The highest molecular weight material showed an enhanced onset of crystallization after flow at a high temperature (200$260\left[{ }^{\circ} \mathrm{C}\right]$ ), while the lower molecular weight material was hardly influenced by flow (for the range of shear rates considered). Applying the same total shear, the influence of shear time and shear rate was compared $(\gamma=\dot{\gamma} \mathrm{t})$. High shear rates in short times were most effective in enhancing crystallization.

\section{Birefringence}

Haudin [57] and Norton and Keller [108] showed that the birefringence of an iPP spherulite depends on the crystallization temperature. Four different types of spherulites were defined (Table 2.5). The birefringence in the $\alpha$ spherulites depend on the 
amount of branches. Suppose that only radial lamellae are created. In this case, the following equation holds:

$$
\Delta=n_{r}-n_{t}=n_{a}-n_{c}<0
$$

with the refractive indices in radial $n_{r}$ and tangential $n_{t}$ direction of the spherulite, and the refractive indices in the direction of the crystal a-axis $n_{a}$ and crystal c-axis $n_{c}$. So, iPP spherulites with radial lamellae show negative birefringence. These spherulites corresponds to the type II spherulites at relative high crystallization temperatures. When lamellar branching occurs the following equation holds (Haudin [57]):

$$
\Delta=\left(\frac{3 t-1}{2}\right)\left(n_{c}-n_{a}\right)
$$

with $t$ the fraction of tangential lamellae. Haudin [57] used the assumption of an orthogonal, uniaxial crystal, which is not true for the biaxial crystals of type I and II for iPP (monoclinic $\alpha$ phase). However, it illustrates that the birefringence depends on the amount of tangential lamellae. With decreasing crystallization temperature, birefringence will change from negative (type II), via mixed, to positive (type I), because the amount of tangential lamellae increases. The $\beta$ related spherulites of types III and IV exhibit a higher magnitude of birefringence. These types correspond to a morphology of parallel stacks and sheets. The high negative birefringence can be explained by the non-branched morphology.

\begin{tabular}{|l||c|c|c|c|}
\hline type & phase & $\Delta \mathrm{n}$ & $\mathrm{T}_{\mathrm{c}}\left[{ }^{\circ} \mathrm{C}\right][57]$ & $\mathrm{T}_{\mathrm{c}}\left[{ }^{\circ} \mathrm{C}\right][108]$ \\
\hline \hline I & $\alpha$ & Weakly positive & $\leq 134$ & $\leq 137$ \\
mixed & $\alpha$ & mixed & $134-138$ & $\leq 140$ \\
II & $\alpha$ & Weakly negative & $\geq 138$ & $\geq 136$ \\
\hline III & $\beta$ & Negative & $110-128$ & $\leq 142$ \\
IV & $\beta$ & Negative & $128-132$ & $128-132$ \\
\hline
\end{tabular}

Table 2.5: Birefringence $\Delta \mathrm{n}$ and crystallization temperatures $\mathrm{T}_{\mathrm{c}}$ of four different iPP spherulites. Data were taken from Haudin [57], Norton and Keller [108].

Jerschow and Janeschitz-Kriegl [66] and Kumaraswamy et al. [76] used birefringence to measure the onset and growth of oriented structures in a slit flow. Jerschow observed birefringence in the highly oriented layers and not in the so called fine grained layers. An increase in turbidity was shown in both layers. Same results were obtained by Kumaraswamy et al. [76]. Using a wall shear rate of 0.06 [Mpa] and shear times larger than 5 [s] at a temperature of $141\left[{ }^{\circ} \mathrm{C}\right]$, oriented structures were obtained. For shear times shorter than 5 [s] or for a lower shear stress (0.03 [Mpa]) crystallization was enhanced, but no oriented structures and corresponding observations could be seen.

Kumaraswamy et al. [76] showed further that the upswing during flow developed at shorter shearing times with increasing temperature. The start of this upswing could be scaled with a shift factor, which showed correlation with the rheological WLF shift factor. The upswing was observed for temperatures up to $175\left[{ }^{\circ} \mathrm{C}\right]$. Upon cessation of shear, the 'shear-induced structure' decayed to a nonzero value at temperatures below $170\left[{ }^{\circ} \mathrm{C}\right]$ while it decayed completely for temperatures larger than $170\left[{ }^{\circ} \mathrm{C}\right]$. 


\begin{tabular}{|c|c|c|c|c|c|c|c|c|}
\hline \multirow{3}{*}{ angle } & \multicolumn{8}{|c|}{ Phases in isotactic PP } \\
\hline & \multicolumn{2}{|l|}{$\alpha[24]$} & \multicolumn{2}{|c|}{$\beta[69]$} & \multicolumn{2}{|c|}{$\gamma[24]$} & \multicolumn{2}{|c|}{ meso [22] } \\
\hline & (hkl) & $\mathrm{I}$ & (hkl) & $\mathrm{I}$ & (hkl) & $\mathrm{I}$ & (hkl) & $\mathrm{I}$ \\
\hline 13.86 & & & & & (111) & $\mathrm{m}$ & & \\
\hline 14.14 & $(110)$ & VS & & & & & & \\
\hline 14.8 & & & & & & & $x$ & $\mathrm{~m}$ \\
\hline 15.11 & & & & & (113) & $\mathrm{W}$ & & \\
\hline 16.10 & & & (300) & VS & & & & \\
\hline 16.92 & $(040)$ & VS & & & & & & \\
\hline 17.02 & & & & & $(008)$ & $\mathrm{s}$ & & \\
\hline 17.35 & & & & & $(115)$ & $\mathrm{W}$ & & \\
\hline 18.55 & $(130)$ & $\mathrm{s}$ & & & & & & \\
\hline 20.27 & & & & & $(117)$ & $\mathrm{m}$ & & \\
\hline 21.31 & $(111)$ & $\mathrm{S}$ & & & $(202)$ & $\mathrm{S}$ & & \\
\hline 21.8 & & & & & & & $x$ & $\mathrm{~m}$ \\
\hline 21.86 & $(131),(041)$ & $\mathrm{s}$ & & & & & & \\
\hline 21.97 & & & & & $(026)$ & $\mathrm{S}$ & & \\
\hline
\end{tabular}

Table 2.6: WAXS reflections $\left(2 \theta_{\mathrm{Cu}} ; \lambda=0.154[\mathrm{~nm}]\right)$ for the four phases in polypropylene. $\mathrm{I}=$ intensity; $\mathrm{vs}=$ very strong; $\mathrm{s}=$ strong; $\mathrm{m}=$ medium; $\mathrm{w}=$ weak. The planes of $\beta$ were defined with a hexagonal unit cell. Data were taken from Brückner et al. [22], Clark [24], Kalay et al. [69].

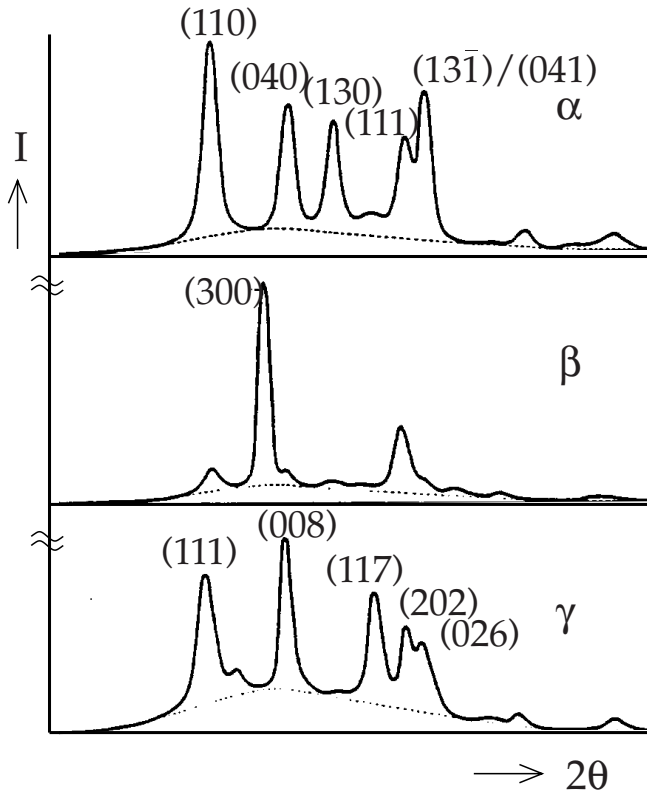

Figure 2.4: WAXS intensity versus $2 \theta$ for three iPP phases. 


\section{Microscopy}

Growth rates appear constant on an optical scale. However, Hobbs et al. [60] showed that lamellar growth appears discontinuous in PHB/V-spherulites using atomic force microscopy. Lamellae initially spurt forward at a rate substantially faster than the macroscopic growth rate, and then slow down or stop. This discontinuity will not be modeled (see next chapter for modeling). The mean growth rate will be determined using optical microscopy. All the different types of spherulites discussed in the previous section will show the Maltese cross, and only type IV will show ringed spherulites between crossed polarizers (Samuels and Yang Yee [125]).

Microscopy studies on shish-kebab structures in iPP were done by Monks et al. [104], White and Bassett [151, 152]. The lamellae that were nucleated on the shish, are generally parallel, not in contact and separated by a few nanometer. Between these lamellae, minute twinned or cross-hatching lamellae were developed. Lamellae parallel to the core increased in number at greater radial distances. Moreover, the growth rates, lamellar thicknesses and cross-hatching were the same for spherulites and for transcrystalline layers. As already discussed, cross-hatching depends on crystallization temperature (see birefringence part of this section). The amount of branches decreased for lower tacticity samples.

In-situ growth of shish-kebab structures of PE was visualized by Hobbs and Miles [61] using atomic force microscopy (AFM). It was observed that lamellae were not equally spaced along the backbone and could adjust their direction slightly upon approaching each other.

\section{WAXS}

The reflections of the four phases of iPP are given in Table 2.6. The intensities of these reflections are graded as (very) strong, medium and weak. The corresponding one dimensional WAXS intensity versus scattering angle patterns are shown in Figure 2.4.

Anisotropic crystalline structures show pronounced arcing in two-dimensional WAXS patterns. The relation between superstructures and (oriented) WAXS reflections is not straight forward, because WAXS provides only information on the crystallite level. Information on this relation can be found in literature and is discussed below.

The branching behavior of $\alpha$-iPP was clearly shown by Lovinger [85] in a thin sample, crystallized on mica. The angle in the (110) reflections was equal to 80/100 degrees, which is equal to the $\beta$-angle in the monoclinic unit cell.

Different types of orientation in $\alpha$-iPP found in literature are shown in Figure 2.5. Increasing orientation corresponds to an increase of orientation in (110) and (130). Nadella et al. [105] correlated the decrease in the corresponding arc lengths with an increase in the molecular weight. An off-equator arcing in (110) and (130) corresponds to the daughter lamellae, which grow in the $\mathrm{a}^{*}$ direction (or flow direction, see Figure 2.6). As described in the introduction part of this section, the b-axis is unaffected in a branched structure. A rotation around the b-axis will only affect the non(0k0) planes (in this case (110) and (130)). This explains the orientation in (040) in Figure 2.5(b-c), while the structure is branched. In highly oriented samples (Figure 2.5(d)), 


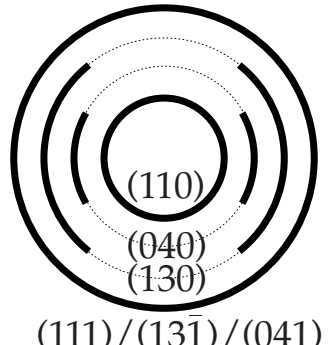

(a)

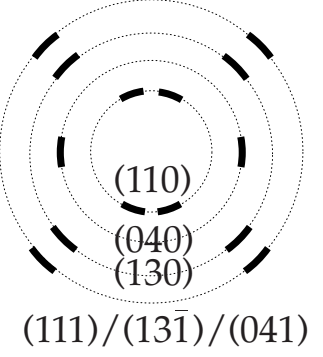

(b)

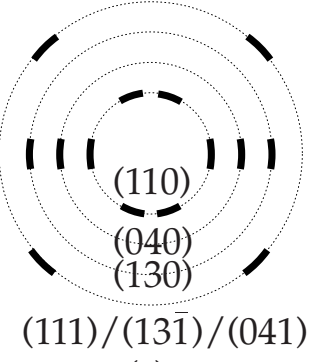

(c)

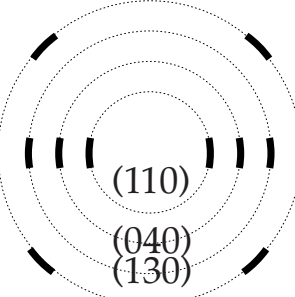

$(111) /(13 \overline{1}) /(041)$

(d)

Figure 2.5: Two-dimensional WAXS patterns of $\alpha$-iPP. Flow direction was vertical. Sample orientation increases from (a) to (d). (a) Core of an injection moulded sample (Fujiyama [41], Fujiyama and Wakino [42], Kalay et al. [68]). (b) Oriented part of $\alpha$ spherulite (Norton and Keller [108]) and unidirectional crystallized sample (Lovinger [85]). (c) Skin of an injection moulded sample (Fujiyama [41], Fujiyama and Wakino [42], SCORIM (Shear Controlled Orientation Injection Moulding) sample (Kalay et al. [68]), during melt spinning (Nadella et al. [105], stretching above the melting temperature (Andersen and Carr [3]) and a short time shearing experiment at 141 [ $\left.{ }^{\circ} \mathrm{C}\right]$ (Kumaraswamy et al. [77]). (d) Stretched near the melting temperature (Andersen and Carr [3]).

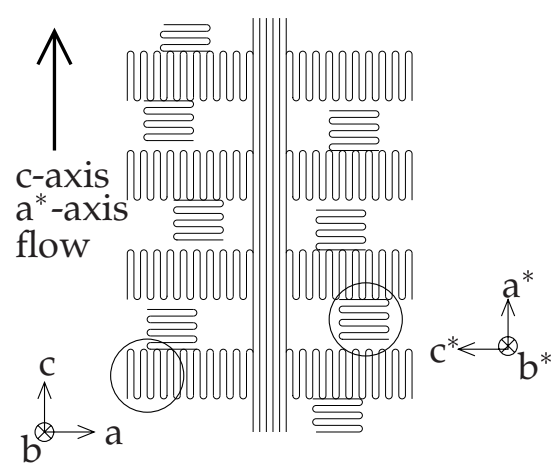

Figure 2.6: Proposed lamellar branched shish-kebab structure in the skin of injection moulded sample (Fujiyama [41], Fujiyama and Wakino [42]). Flow direction is vertical.

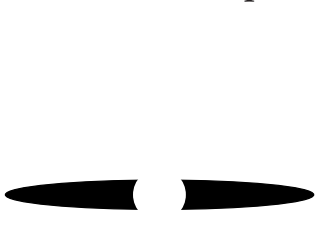

(a)
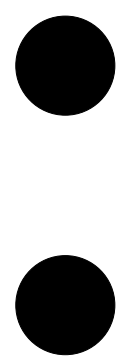

(b)

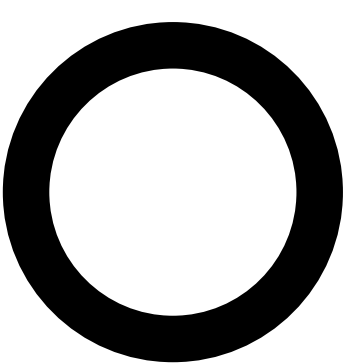

(c)

Figure 2.7: Two-dimensional SAXS patterns in time (from (a) to (c)). Flow direction was vertical. Intermediate stages are not drawn. (a) Equatorial streak at the initial stage of melt spinning for PB, PE and PVDF (Samon et al. [120, 121]). (b) Meridional lobes after shearing of iPP (Somani et al. [134]), during melt spinning of iPP (Nadella et al. [105]), during melt spinning of PE, PB and PVDF (Samon et al. [120, 121]) and in the skin of an injection moulded sample of iPP (Fujiyama and Wakino [42]). (c) Isotropic ring after shearing of iPP (Somani et al. [134]) and in the center of an injection moulded sample of iPP (Fujiyama and Wakino [42]). 
a fiber-like structure is seen with equatorial reflections in (110), (040) and (130). Fujiyama proposed a structure as given in Figure 2.6 corresponding to the WAXS pattern in Figure 2.5(c). It is a branched shish-kebab structure. This agrees with the observations of Monks et al. [104], White and Bassett [152] (see Microscopy part of this section).

\section{SAXS}

Somani et al. [134] followed crystallization after a shear step in a Linkam shear cell, using in-situ synchrotron SAXS. Meridional lobes corresponding to the kebab structure, were observed immediately after the step in shear (flow direction was vertical). Next, an isotropic scattering ring starts to grow, which corresponds to the growth of unoriented crystallites. Both SAXS and TEM indicate no evidence of primary oriented nuclei and the resulting shish structures. The long period decreases rapidly with time in the initial stages of crystallization. At the later stages, the long period decreases slowly. The first stage was ascribed to the insertion of new lamellae and the second stage to secondary crystallization.

The meridional lobes were also observed by Fujiyama and Wakino [42], in the skin of an injection moulded sample, and Nadella et al. [105] during melt spinning at high speeds. Fujiyama found no equatorial lobe caused by the daughter lamellae (Figure 2.6). He concluded that these $a^{*}$ oriented lamellae are small and imperfect. An isotropic ring, corresponding to random orientation of lamellae, was observed by Fujiyama in the core of an injection moulded sample, and by Nadella at low spinning speeds.

Samon et al. [121] followed the early stages of crystallization during melt spinning of PE and PVDF by in-situ synchrotron SAXS/WAXS. An equatorial streak, corresponding to shish formation (Samon et al. [120]), was observed in these measurements. Next, meridional lobes, corresponding to kebab formation, were observed in SAXS. Finally, the equatorial streak diminished due to the decreasing contrast between the shishes and growing kebabs or due to destruction of the shish structure. This last explanation agrees with the observations of Schultz and Petermann [132], who saw a transformation of fibrils into lamellae during heat treatment (at $150\left[{ }^{\circ} \mathrm{C}\right]$ ) of a melt drawn polypropylene.

\subsection{Measurements of flow-induced nucleation and crys- tallization}

A short review on measurements of flow-induced nucleation and crystallization is given. Previous reviews can be found in Eder et al. [36], McHugh [95] and Tribout et al. [140]. Here, special attention is paid to measurements of the last decennium. The standard characterization methods were already described in the previous section.

References on flow-induced crystallization together with the method, device and material used are shown in Table 2.7. Attention is paid to the experimental device, the settings and typical results. 


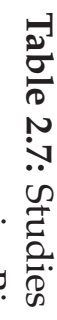

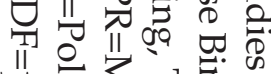
光运軍富

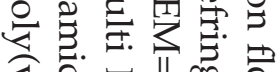

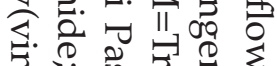

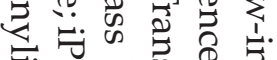

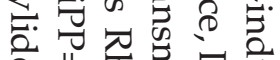
造 $\frac{11}{\omega}$.

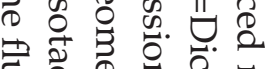
总. 웡 四

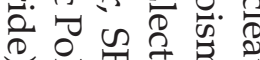

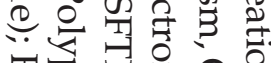
검

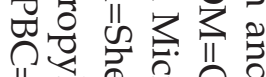
II

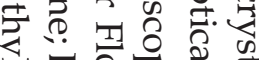

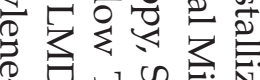
i

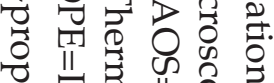
는.

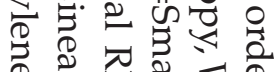

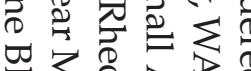

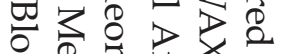
수을 ○家过 $\checkmark$ U 证

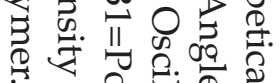
记

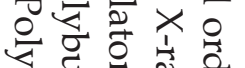

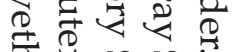
运 宁总串

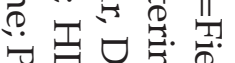

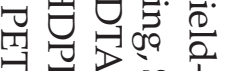
III III 농료

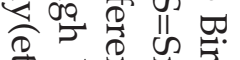
承 贾. 蛋 ( $)$

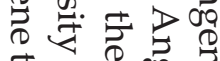

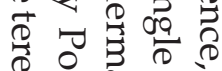

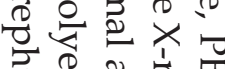

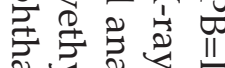

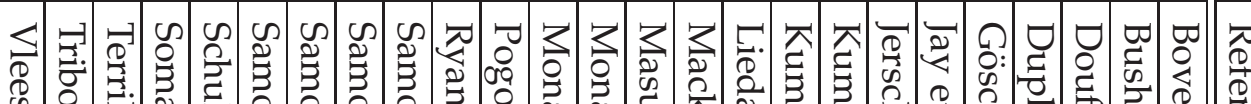

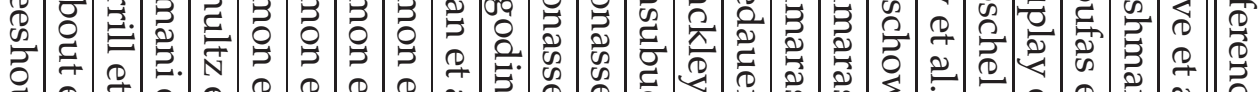

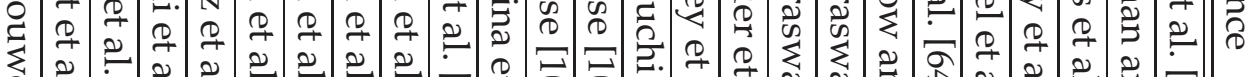

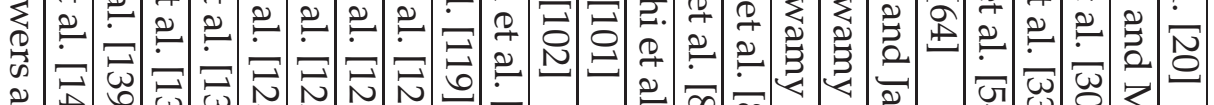

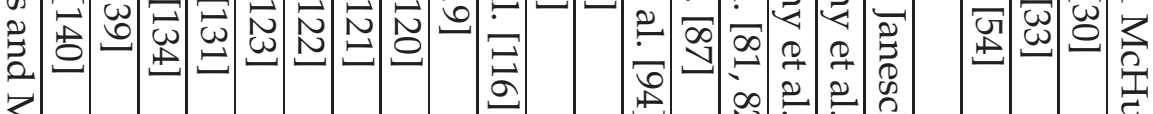
苗:

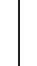

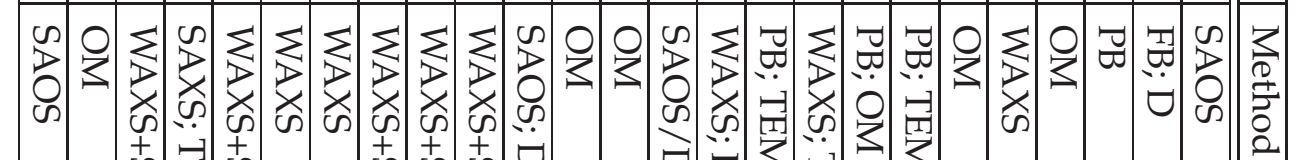

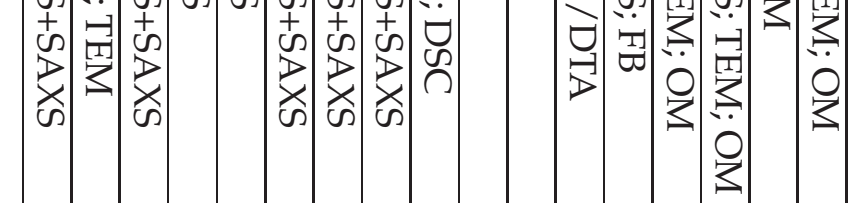

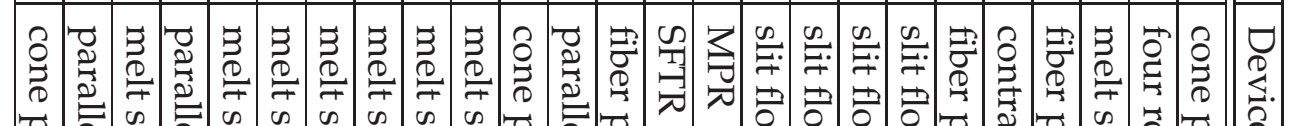

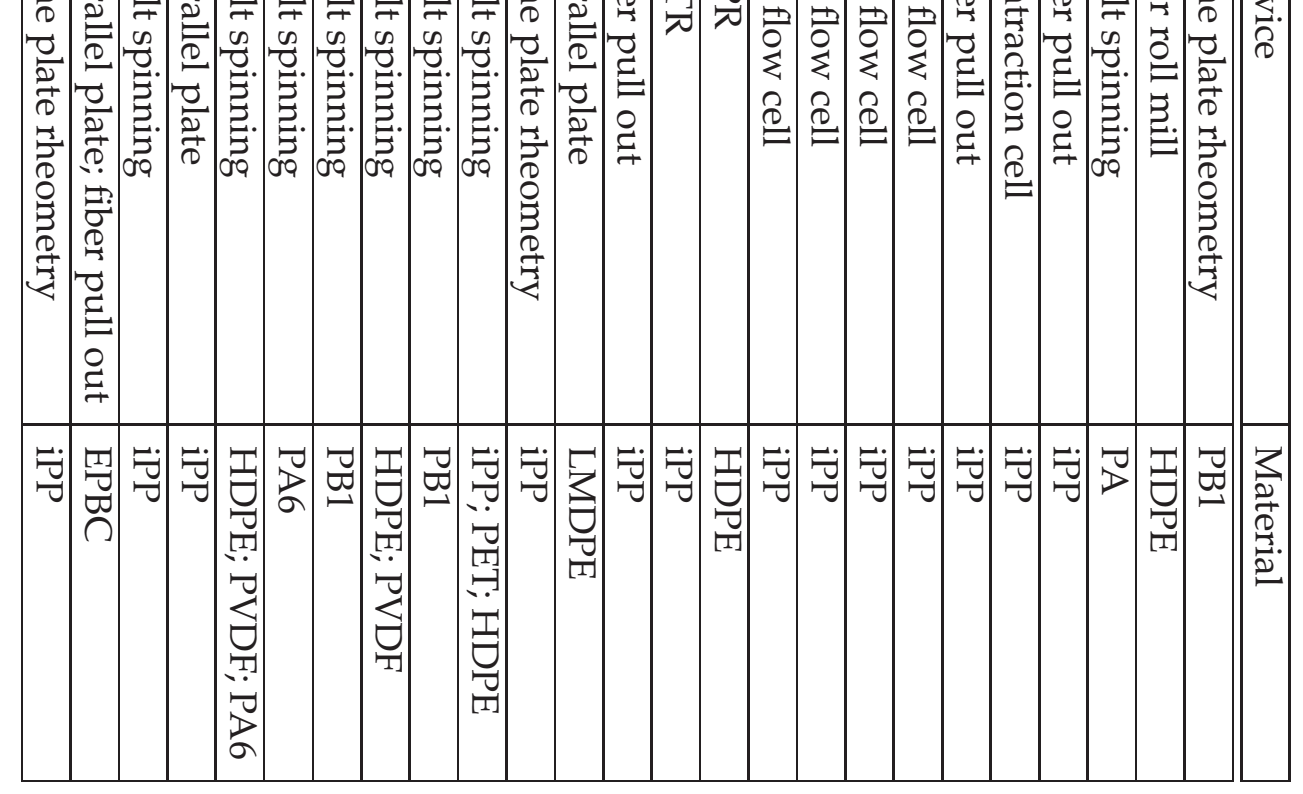

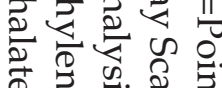

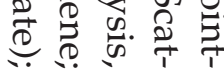




\section{Devices}

About eight different experimental devices were used to measure flow-induced nucleation and crystallization (see Table 2.7). Shear flow was applied in the fiber pull out device, between parallel plates, in slits, in the Multi Pass Rheometer (MPR) and in shear or stress controlled rheometers. The four roll mill was the only device in which (local) pure elongation (i.e. $\dot{\varepsilon}=$ constant) was applied. Uniaxial elongational flow is the most strongly orienting flow. The melt spinning apparatus and the contraction flow cell can be considered as complex flows with a combination of shear and elongation. This division is made on basis of the macroscopical flow field. On a molecular level, polymers can be stretched, also as a consequence of shear, provided that the shear and shear rate are high enough. Only the MPR and the melt spinning experiments approached the industrial processing conditions. Spinning speeds up to $8.3[\mathrm{~m} / \mathrm{s}]$ (Samon et al. [123], Schultz et al. [131]) and shear rates up to 2225 [s $^{-1}$ ] (MPR, Mackley et al. [87]) were reported.

The fiber pull out device used by Haudin and coworkers consisted of a hot-stage and a sample containing a fiber, which can be pulled out. Fiber diameter was about $17[\mu \mathrm{m}])$ and sample thickness was about 0.2-0.3 [mm]. Morphology was investigated in-situ using a microscope. The limitation in this method is the deformation history, which depends on the length of the fiber. Shear rates up to $400\left[\mathrm{~s}^{-1}\right]$ were reported in Monasse [101].

Two different parallel plate set-ups were described, namely a home-made set-up (Monasse [102]) and a commercially available Linkam shear cell (Linkam CSS-450, Somani et al. [134]). The device of Monasse consisted of two parallel glass plates, both heated with a hot plate. The lower plates could move in longitudinal direction. Morphology was investigated in-situ using a microscope. Sample thickness was about $0.3[\mathrm{~mm}]$. Reported shear rates were less than $4.2\left[\mathrm{~s}^{-1}\right]$. Somani used rotating parallel plates with ring shaped samples having an inner and outer diameter of 10 and 20 [mm], respectively, and a sample thickness of 0.5-1.0 [mm]. Reported shear rates were less than $102\left[\mathrm{~s}^{-1}\right]$. Drawback of this set-up is the radius depended deformation history.

Liedauer et al. [81, 82], Jerschow and Janeschitz-Kriegl [66, 67] and Kumaraswamy et al. [76, 77] used the same type of slit. Short time shearing experiments were performed in a rectangular duct $\left(10 \times 1\left[\mathrm{~mm}^{2}\right]\right.$ (Liedauer $/$ Jerschow) and $5 \times 0.5\left[\mathrm{~mm}^{2}\right](\mathrm{Ku}-$ maraswamy)), in which a box-like pressure-time profile was created. The flow cell of Liedauer and Jerschow was mounted behind a single-screw extruder. An accumulator and a bypass were used to create the desired constant pressure. It is unknown how flow was created in the flow cell of Kumaraswamy. Maximum reported shear rates in the device of Liedauer/Jerschow and Kumaraswamy were $195\left[\mathrm{~s}^{-1}\right]$ and $10\left[\mathrm{~s}^{-1}\right]$ respectively. The Multi Pass Rheometer (MPR) of Mackley et al. [87] consisted of two hydraulic cylinders in between which a flow geometry (e.g. a slit) can be inserted. Also push pull flow can be created in this flow cell by moving the pistons periodically forward and backward. Mackley used a slit of $5 \times 1 \times 1\left[\mathrm{~mm}^{3}\right]$.

Standard rheometers were used by Bove et al. [20], Pogodina et al. [116], Vleeshouwers and Meijer [147]. Masubuchi et al. [94], Nagatake et al. [106] developed a shear flow thermal rheometer, a standard shear controlled rheometer in which differential 
thermal analysis (DTA) was incorporated. Bove, Vleeshouwers and Masubuchi used a shear controlled rheometer and Pogodina used a stress controlled rheometer. Maximum reported shear rates were $5\left[\mathrm{~s}^{-1}\right]$ (Bove), $10\left[\mathrm{~s}^{-1}\right]$ (Vleeshouwers), $1\left[\mathrm{~s}^{-1}\right]$ (Masubuchi) and about $2\left[\mathrm{~s}^{-1}\right]$ (Pogodina; estimated by assuming a viscosity between $1 \cdot 10^{4}$ and $1 \cdot 10^{5}[\mathrm{~Pa} \mathrm{~s}]$ at $\left.140\left[{ }^{\circ} \mathrm{C}\right]\right)$.

Bushman and McHugh [23], McHugh et al. [96] investigated a high molecular weight HDPE-droplet in the four roll mill. A linear low density polyethylene carrier fluid was used to reduce elastic stresses in order to prevent blockage of the mill. The difference in refraction of those two fluids increases the complexity of the birefringence measurements. The 19 [mm] diameter rolls are positioned at the corners of a 34.9 [mm] square and connected with a motor. Depth of the device was equal to 10.2 [mm]. Elongational rates were less than $0.05\left[\mathrm{~s}^{-1}\right]$.

In the melt spinning experiments an extruder was used, which could be translated in vertical direction in order to perform in-situ X-ray scattering experiments on different fiber positions. The polymer was extruded through a single hole die (diameter $=$ 1/32 inch (Samon et al. [120]), through a rectangular die $\left(4 \times 2\right.$ [ $\left.\mathrm{mm}^{2}\right]$ Schultz et al. [131] and $3 \times 0.5\left[\mathrm{~mm}^{2}\right]$ Ryan et al. [119]) or through a six hole multifilament die with a hole diameter of 1.5 [mm] (Samon et al. [121, 122], Schultz et al. [131]. The advantage of this set-up is the high winding speeds. The disadvantage is the complex deformation and thermal history. Samon et al. [122] used an IR camera to measure temperatures of the fiber. Furthermore, the complicated mechanism of secondary nucleation or growth during flow, especially for these extreme flow conditions, is still not clear.

The contraction cell described in Göschel et al. [54] consisted of a cylindrical reservoir (diameter $9.5[\mathrm{~mm}])$ to a rectangular channel contraction $\left(2 \times 1.5\left[\mathrm{~mm}^{2}\right]\right)$. Details were described in Bilsen et al. [12]. Flow was created by using a hydraulic cylinder which moves a piston in the reservoir. Shear rates up to $127\left[\mathrm{~s}^{-1}\right]$ were reported. Disadvantage of this set-up is the complex flow in the contraction region and the circulating flow in front of the piston. Therefore, the deformation history in this cell is badly defined.

\section{Measurements}

Liedauer et al. [81, 82] were the first who found a quantitative relation between the morphology and a shear-induced crystallization model (see also Eder and JaneschitzKriegl [35], Jerschow and Janeschitz-Kriegl [66, 67]). Their success was based on a rather simple but clear model (see Chapter 3) and well defined experiments. Short time shearing at low supercooling was used. Flow time was short enough, and flow temperature was high enough, such that growth of the sporadic created nuclei was negligible with respect to the crystallization time. Shearing time was a few percentage of the total crystallization time.

Liedauer found three different regions over the thickness of the duct: oriented structures close to the wall, spherulites in the center and a fine grained layer in between. A correlation between the rate of crystallization and the amount of shearing was found and was equal to $\dot{\gamma}^{4} \mathrm{t}_{\mathrm{s}}^{2}$. The temperature dependence of the critical shear rates at which the boundaries of the three different layers were found was not pronounced. According to Jerschow and Janeschitz-Kriegl [66] the structures in the fine 
grained layer were thread-like particles which were oriented perpendicular to flow direction. Jerschow explained that this orientation in the neutral direction could be due to the second normal stress difference. It was stated that if shear rate is sufficiently high, these structures can rotate into the flow direction.

Most references in Table 2.7 applied the short time shearing approach at low supercooling, where flow and crystallization temperature were taken equal. The melt spinning experiments and the experiments of Monasse [101] and Vleeshouwers and Meijer [147] are an exception. In the melt spinning experiments a flow temperature of about forty degrees above the (non-equilibrium) melting temperature was applied. Flow temperatures in the work of Vleeshouwers on iPP were in between 200 and $260\left[{ }^{\circ} \mathrm{C}\right.$ ]. Monasse used flow temperatures of 170 and $210\left[{ }^{\circ} \mathrm{C}\right]$ for iPP. Relaxation of the material in these experiments can have a large influence on the occurrence of oriented structures, especially if the cooling rate is not large enough.

Disadvantage of the short time shearing protocol at relatively low temperatures could be the interaction of formed structures with flow. Crystalline structures can appear during flow like in Kumaraswamy et al. [77], who saw an oriented WAXS pattern during the first 5 [s] of shearing. The peaks grew rapidly until 100 [s], after which they saturate and grow relatively slowly. Mackley et al. [87] observed after 10 [s] shearing of HDPE in the MPR at $135\left[{ }^{\circ} \mathrm{C}\right]$ and $225\left[\mathrm{~s}^{-1}\right]$ an increase in pressure and formation of fibers. Typically, these oriented structures were not observed at a higher shear rate of $2250\left[\mathrm{~s}^{-1}\right]$. According to Mackley slip at the wall suppressed crystallization at this high shear rate.

Secondary nucleation or growth was investigated in the fiber pull out device. Duplay et al. [33] investigated fourteen different polypropylene homopolymers, which differ in molecular weight and molecular weight distribution, but with the same tacticity. It was shown that that spherulitical growth of iPP was not influenced by molecular weight. Similar results were obtained by Eder et al. [36] and Gahleitner et al. [45]. Magill [90] reported that the growth rate can be dependent on molecular weight, but this dependence becomes less for higher molecular weights. The decrease of growth rate with decreasing tacticity was shown by Gahleitner et al. [45]. Duplay showed that growth rate was increased during shear and correlated with molecular weight. The largest deviation was found for a high molecular weight with a broad molecular weight distribution $\left(\mathrm{M}_{\mathrm{w}}=375[\mathrm{~kg} / \mathrm{mol}]\right.$ and $\left.\mathrm{MWD}=13.2[-]\right)$. In this case the growth rate during shear was about ten times higher than in quiescent conditions. Also Tribout et al. [140] showed a shear dependent growth rate. The growth rates during shear were about five times and after shear about 1.5 times higher than in the static case. The growth rate increased linearly with increasing shear rate. In conclusion, tacticity seems to be the most important molecular parameter on growth in quiescent conditions at a constant temperature. Moreover, growth rate after shear is slightly dependent on the deformation history while during shear it correlates with molecular weight.

Bushman and McHugh [23], McHugh et al. [96] investigated the extensional flow of an UHWPE droplet in a four roll mill using point-wise birefringence and dichroism measurements on the outflow centerline. Flow birefringence showed an increase in orientation with distance from the stagnation point. This corresponds to the first normal stress difference. After cessation of flow, birefringence showed complete relaxation of the carrier phase, while birefringence of the droplet phase showed retention 
of orientation and/or development of crystallinity. The maximum in birefringence intensity occurred first at a position of about $10[\mathrm{~mm}]$ from the stagnation point. The time to obtain this maximum increased for decreasing distance from the stagnation point. Initial crystallization was seen to be a function of both orientation and strain by comparing the behavior on the outflow centerline points. It was suggested that the viscoelastic molecular strain due to flow orientation might be the controlling process for enhancing crystallization.

One of the few studies on the influence of nucleation agents on flow-induced crystallization was by Jerschow and Janeschitz-Kriegl [67]. Nucleating agents strongly enhanced shear-induced crystallization. Very thick, high oriented layers far into the core were found even for low molecular weight materials.

The strain-induced crystallization studies of Blundell et al. [14, 15, 16] and Mahendrasingam et al. [91, 92, 93] are closely related with the flow-induced crystallization studies described above. In-situ WAXS experiments were performed at the microfocus beam-line of the ESRF on fast drawing of PET close to $\mathrm{T}_{\mathrm{g}}$. Three different regimes could be observed (Blundell et al. [15]). Details of the experimental conditions of these regimes are given in Mahendrasingam et al. [91, Figure 11]. In the first regime, corresponding to low temperatures and high draw rates, orientation was insensitive to draw rate and was mainly in drawing direction. The onset of crystallization was delayed until the end of deformation. In the third regime, corresponding to high temperatures and low draw rates, no oriented structures were observed. In the second regime, corresponding to intermediate temperatures and draw rates, orientation decreased with increasing temperature and decreasing draw rate, and was tilted with respect to the drawing direction. The tilting in the orientation was explained by the interaction of the nuclei formed with the surrounding network, which causes a tilt at lower draw rates or higher temperatures. The onset of crystallization could be seen before the end of drawing. The difference in onset in the first two regimes was ascribed to the lack of freedom and mobility to organize chains into crystals during deformation at high deformation rates.

The strain-induced crystallization rate was found to be insensitive to temperature (Blundell et al. [14]). Two processes with opposing temperature dependence were found to be involved (Blundell et al. [15], Mahendrasingam et al. [91]). An increase in temperature reduced the crystallization rate as a result of lower network orientation for a given deformation rate. This was counteracted by the effects of increased molecular mobility on the crystallization kinetics.

\section{SAXS before WAXS}

The appearance of SAXS before WAXS caused many discussions. This observation was already reported in 1977 by Nogami et al. (see the review of Eder et al. [36]). Lately, many researchers observed the same in advanced synchrotron radiation facilities by using simultaneous SAXS/WAXS, in the melt spinning experiments (e.g. Ryan et al. [119], Samon et al. [121], Schultz et al. [131], Terrill et al. [139]).

Ryan et al. [119], Terrill et al. [139]) saw tear shaped scattering in SAXS with only an amorphous halo in WAXS close to the die head. The peak moves to higher scattering angles (SAXS) with the onset of crystal growth in WAXS. At the furthest position of 
the die head, isotropic rings were seen both in WAXS and SAXS. Samon et al. [121] and Schultz et al. [131] observed an equatorial streak in SAXS at the closest position to the die, while an amorphous hallow was found in WAXS. Meridional lobes in SAXS and crystalline peaks in WAXS were seen further away from the die.

An controversial explanation of SAXS occurring before WAXS, namely spinodal decomposition of the crystalline and amorphous phase, was suggested by Ryan et al. [119] and Terrill et al. [139]. Pre-nucleation density fluctuations was suggested to play an important role in nucleation of polymer crystallization. Consequently, changes in conformation followed by crystallization occur sequentially. This is the main difference with classical nucleation and growth, where those two processes are assumed to occur simultaneously. Once crystallization had been observed in the wide-angle region, the shape of the small-angle pattern changed from that characteristic of sinusoidal density fluctuations to that typical of lamellar crystals, and the kinetics reverted to those of nucleation and growth.

Mahendrasingam et al. [93] observed a weak meridional peak for a very short time in WAXS during drawing of PET close to $\mathrm{T}_{\mathrm{g}}$ for high draw rates. The peak corresponded to a mesophase. Mahendrasingam concluded that polymer crystallization may be assisted by the formation of this intermediate meta-stable state, which is described by the spinodal composition theory.

The discrepancy in onset times was investigated by Wang et al. [148, 149] for quiescent crystallization of PE and PP. Besides WAXS and SAXS also light scattering was used. Wang observed a method dependent detection limit of crystallinity. Light scattering was the most sensitive $(0.1 \%)$, followed by SAXS (about $0.8 \%$ ) and WAXS $(2 \%)$. The crystallinities found by these three methods could be described by a single Avrami equation, suggesting classical nucleation and growth. It was concluded that the difference in onset time depends on the difference in the detection limit of the experimental methods.

A problem in comparing the two methods is the difference in intensity level and the constructive interference in WAXS. Intensity depends strongly on scattering angle. Intensity is much higher close to the beam-stop than in the WAXS region. The constructive interference condition in WAXS, which demands nicely stacked lattices, seems to be much severe than the density fluctuations necessary in SAXS. For these two reasons, SAXS should be more sensitive than WAXS. Bras [21] checked the sensitivity of WAXS by comparing measurements with a more sensitive WAXS detector with previous measurements. A smaller difference in the onset time between WAXS and SAXS was observed, but still SAXS appeared before WAXS.

\subsection{Discussion}

Polymorphism of isotactic polypropylene was well investigated in literature. The monoclinic $\alpha$-phase is the most stable form and will be obtained under 'standard crystallization conditions'. Lamellar branching of the $\alpha$-phase was observed in microscopy. The amount of branching depends both on the thermal and deformation history and can be measured with birefringence. In WAXS branching influences the orientation of the (110) and (130) reflections. Consequently, orientation was not only found at 
the equator. The small imperfect branched lamellae in high oriented regions were not observed in SAXS. The shish structure was only observed in SAXS by Samon et al. [121], Schultz et al. [131] as an equatorial streak.

Differences in nucleation and growth behavior of the different crystalline phases in iPP, especially the $\alpha$ - and $\beta$-phase (the amount of $\gamma$ is negligible for the high isotacticities used in this study), enlarge difficulties in modeling flow-induced nucleation and crystallization, because a large amount of experimental input data is needed. The determination of nucleation and growth for the $\alpha$-phase in a certain iPP is much easier than for the $\beta$-phase, because the $\beta$-phase is more rare.

Most of the experimental studies used the short time shearing protocol at low supercoolings. Pure elongational flow was only investigated by Bushman and McHugh [23], McHugh et al. [96]. However, their experimental conditions are complex due to the use of a droplet in a carrier. Reported elongational rates were small. The behavior of flow-induced crystallization at higher elongational rates is quantitatively still unknown. Elongational rates are high in the melt spinning experiments, which approach industrial conditions, but the deformation and thermal history in these experiments are not well defined.

The exact nucleation mechanism is still unresolved. The stages of growth of structures was shown nicely in the time resolved synchrotron WAXS/SAXS studies. Differences in the onset of crystallization found by these studies could be explained by a difference in sensitivity. Therefore, to resolve the initial stages of crystallization sensitive new experimental methods are required. 


\section{Chapter 3}

\section{Constitutive models}

In this chapter constitutive models are given to describe stress, and stress-induced nucleation and crystallization. Coupling with crystallization during and after flow is formulated by a dependence of morphology development on applied stress.

A generalized Newtonian model and three non-linear viscoelastic equations for the stress tensor are discussed in Section 3.1. The generalized Newtonian model can give an accurate prediction of the kinematics of the flow when this is shear dominated. Non-linear viscoelastic equations are necessary to give the viscoelastic stresses of polymeric liquids, both in shear and elongation. Most models are unable to predict stresses quantitatively in both flows. The Pompom model is an exception and fits elongation and shear data excellent. More details on constitutive models and modeling can be found in Bird et al. [13] and Larson [78, 79].

Constitutive equations to describe stress-induced crystallization are described in Section 3.2. A set of differential equations are given for crystallization in quiescent conditions (Schneider rate equations) and crystallization after flow $\left(\mathrm{S}_{\mathrm{J} 2}\right.$-model). Nucleation in the $S_{\mathrm{J} 2}$-model is coupled with viscoelastic stress via the recoverable strain.

Only differential equations are considered, because these equations can be implemented easily in numerical schemes.

\subsection{Constitutive equations for the stress tensor}

\subsubsection{Generalized Newtonian model}

A generalized Newtonian model describes the shear rate dependence of the viscosity accurately. However, it is unable to predict (transient) non-Newtonian stresses.

The constitutive equation for the symmetric Cauchy stress tensor for incompressible fluids is normally splitted in a hydrostatic (-pI) and a deviatoric part

$$
\boldsymbol{\sigma}=-p \boldsymbol{I}+\boldsymbol{\tau}
$$

The extra stress $\tau$ for generalized Newtonian models depends on the rate of deformation via the second invariant of the deformation tensor (Bird et al. [13]).

$$
\boldsymbol{\tau}=2 \eta\left(\mathcal{I I}_{2 \boldsymbol{D}}\right) \boldsymbol{D}
$$


with the deformation tensor $\mathbf{D}$, which is the symmetric part of the velocity gradient tensor $\mathbf{L}$, and the second invariant $\mathcal{I} \mathcal{I}$ of $2 \mathrm{D}$. The deformation and gradient tensor, and the second invariant are defined as

$$
\boldsymbol{D}=\frac{1}{2}\left(\boldsymbol{L}+\boldsymbol{L}^{T}\right) \quad \boldsymbol{L}=(\vec{\nabla} \vec{v})^{T} \quad \mathcal{I I}_{\boldsymbol{A}}=\frac{1}{2}\left(\operatorname{tr}(\boldsymbol{A})^{2}-\operatorname{tr}(\boldsymbol{A} \cdot \boldsymbol{A})\right)
$$

An example of a generalized Newtonian model is the Carreau-Yasuda model, which is used in Chapter 5 to calculate the three-dimensional velocity field in the cross-slot device:

$$
\frac{\eta-\eta_{\infty}}{\eta_{0}-\eta_{\infty}}=\left(1+\left(\lambda\left|\mathcal{I I}_{2 \boldsymbol{D}}\right|^{\frac{1}{2}}\right)^{a}\right)^{\frac{n-1}{a}}
$$

with the zero shear-rate viscosity $\eta_{0}$, the infinite shear-rate viscosity $\eta_{\infty}$, the relaxation time $\lambda$ and the power law parameter $n$. Parameter a describes the transition region between the zero shear-rate and the power law region. The viscosity $\eta_{\infty}$ is often not taken into account, because it is not measured. A Newtonian model is obtained for $\mathrm{n}=1$ and the Carreau model is obtained for $\mathrm{a}=2$.

\subsubsection{Nonlinear viscoelastic models}

A nonlinear viscoelastic constitutive equation is able to describe non-Newtonian phenomena. Three different models will be discussed. First, a general evolution equation for the extra stress tensor will be derived. Second, the slip tensor will be described for the models. Finally, more detailed information is given on the models.

\section{Evolution equation}

Restricting to elastic dumbbells with a Gaussian probability distribution function, in molecular theories the Cauchy stress tensor is related to the molecular end-to-end vector $\vec{R}$ by

$$
\boldsymbol{\sigma}=a<\vec{R} \vec{R}>=a \boldsymbol{C}
$$

with a constant a depending on molecular parameters (length and number of chains), and the conformation tensor $\mathbf{C}$. The brackets describe the averaging of the end-to-end vector length over the probability distribution function. The change in the length of an end-to-end vector generates a force, which is related to the change of entropy. This behavior is know as 'entropic spring'. The conformation tensor can also be written in terms of the orientation tensor $\mathbf{S}$ :

$$
\boldsymbol{C}=<\vec{R} \vec{R}>=\Lambda^{2}<\vec{n} \vec{n}>=\Lambda^{2} \boldsymbol{S}
$$

with the stretch $\Lambda$ in the direction $\vec{n}$. The time derivative of conformation tensor $C$ is

$$
\dot{\boldsymbol{C}}=<\dot{\vec{R}} \vec{R}>+<\vec{R} \dot{\vec{R}}>
$$


Affine deformation of a polymer chain is described by

$$
\dot{\vec{R}}=\boldsymbol{L} \cdot \vec{R}
$$

A more realistic description for polymer liquids is obtained by introducing a slip tensor A to model the non-affine motion

$$
\dot{\vec{R}}=(\boldsymbol{L}-\boldsymbol{A}) \cdot \vec{R}
$$

This equation is substituted in Equation (3.7) and leads to the stress evolution equation

$$
\stackrel{\nabla}{\boldsymbol{\sigma}}+\boldsymbol{A} \cdot \boldsymbol{\sigma}+\boldsymbol{\sigma} \cdot \boldsymbol{A}^{c}=\boldsymbol{O}
$$

where the upper convective derivative of the Cauchy stress tensor is defined by

$$
\stackrel{\nabla}{\boldsymbol{\sigma}}=\dot{\boldsymbol{\sigma}}-\boldsymbol{L} \cdot \boldsymbol{\sigma}-\boldsymbol{\sigma} \cdot \boldsymbol{L}^{T}
$$

The stress evolution equation for the extra stress tensor can be derived by using $\sigma=$ $\mathrm{GI}+\boldsymbol{\tau}$ and the upper convective derivative of the unity tensor $(\stackrel{\nabla}{\mathbf{I}}=-2 \mathrm{D})$

$$
\stackrel{\nabla}{\boldsymbol{\tau}}+\boldsymbol{A} \cdot \boldsymbol{\tau}+\boldsymbol{\tau} \cdot \boldsymbol{A}^{c}+G\left(\boldsymbol{A}+\boldsymbol{A}^{c}\right)=2 G \boldsymbol{D}
$$

with the modulus G. For a realistic description of melt behavior a multi-mode model has to be used:

$$
\boldsymbol{\sigma}=-p \boldsymbol{I}+\sum_{i=1}^{n} \boldsymbol{\tau}_{i}
$$

with the total number of modes $n$.

\section{Slip tensor}

The slip tensor $\mathbf{A}$ is also known as the plastic deformation rate tensor $\mathbf{D}_{\mathrm{p}}$. Models used in this thesis are captured in the following definition

$$
\boldsymbol{A}=\alpha_{1} \boldsymbol{\sigma}+\alpha_{2} \boldsymbol{\sigma}^{-1}+\alpha_{3} \boldsymbol{I}
$$

by a certain choice for the model parameters $\alpha_{1}, \alpha_{2}$ and $\alpha_{3}$. The constants for the upper convected Maxwell, the Leonov, the Giesekus and the extended Pompom model are described in Table 3.1. Slip tensors for some other popular constitutive equations were given in Peters [111], Peters and Baaijens [113].

The Pompom model has a generalized relaxation time $\lambda_{\mathrm{g}}$ that is described by

$$
\lambda_{g}=\frac{1-\alpha}{\lambda \Lambda^{2}}-\frac{\alpha \mathcal{I}_{\boldsymbol{\sigma} \cdot \boldsymbol{\sigma}}}{3 G^{2} \lambda \Lambda^{2}}+\frac{2}{\lambda_{s}}\left(1-\frac{1}{\Lambda}\right)
$$

with a material parameter $\alpha$, which defines the amount of anisotropy in molecular friction and (Brownian) motion (i.e. reptation). Two different relaxation times are defined, namely the relaxation time of the backbone orientation $\lambda\left(=\lambda_{0 \mathrm{~b}}\right)$ and the backbone stretch $\lambda_{\mathrm{g}}$. The parameter $\alpha$ and the relaxation times are described more detailed in the next section. 


\begin{tabular}{|l||c|c|c|l|}
\hline Model & $\alpha_{1}$ & $\alpha_{2}$ & $\alpha_{3}$ & Ref. \\
\hline \hline UCM & 0 & $-\frac{G}{2 \lambda}$ & $\frac{1}{2 \lambda}$ & [113] \\
\hline Leonov & $\frac{1}{4 G \lambda}$ & $-\frac{G}{4 \lambda}$ & $-\frac{\mathcal{I}_{\boldsymbol{\sigma}}-G^{2} \mathcal{I}_{\boldsymbol{\sigma}}-1}{12 G \lambda}$ & [113] \\
\hline Giesekus & $\frac{\alpha}{2 G \lambda}$ & $-\frac{G(1-\alpha)}{2 \lambda}$ & $\frac{(1-2 \alpha)}{2 \lambda}$ & [113] \\
\hline Extended Pompom & $\frac{\alpha}{2 G \lambda}$ & $-\frac{G(1-\alpha)}{2 \lambda}$ & $\frac{1}{2 \lambda_{g}}$ & [143] \\
\hline
\end{tabular}

Table 3.1: Definition of the slip tensor for the upper convected Maxwell (UCM), the Leonov, the Giesekus and the Extended Pompom model (taken from Peters and Baaijens [113], Verbeeten et al. [143]).

\section{Upper convected Maxwell model}

The upper convected Maxwell (UCM) model can describe different aspects of linear and non-linear viscoelastic behavior of a polymeric fluid by using multi modes. Linear behavior can be predicted accurately, while the non-linear viscoelastic regime is only described qualitatively. It can predict a first normal stress difference in shear, strain hardening in elongation and stress relaxation after cessation of flow. However, shear viscosity and the first normal stress difference are independent of shear, and elongational viscosity can become infinite for finite elongational rates (Larson [78]). The differential equation of the UCM model is given by

$$
\stackrel{\nabla}{\boldsymbol{\tau}}+\frac{1}{\lambda} \boldsymbol{\tau}=2 G \boldsymbol{D}
$$

The UCM model is fully determined using linear viscoelastic data only.

\section{Leonov}

The Leonov model can describe the (non)-linear viscoelastic behavior of a polymeric liquid in shear excellent by using multi modes. Elongation is poorly described by Leonov (Larson [78]). The differential equation of the Leonov model is given by

$$
\stackrel{\nabla}{\boldsymbol{\tau}}+\frac{1}{\lambda} \boldsymbol{\tau}+\frac{1}{2 G \lambda} \boldsymbol{\tau} \cdot \boldsymbol{\tau}-\frac{1}{6 G \lambda} \underbrace{\left[\mathcal{I}_{(\boldsymbol{\tau}+G \boldsymbol{I})}-G^{2} \mathcal{I}_{(\boldsymbol{\tau}+G \boldsymbol{I})^{-1}}\right]}_{(a)}(\boldsymbol{\tau}+G \boldsymbol{I})=2 G \boldsymbol{D}
$$

The Leonov model is, like the UCM model, determined by the linear viscoelastic data only. For incompressible, planar deformations part (a) of Equation (3.17) is equal to zero. The Leonov model is in this case equivalent to the Giesekus model with $\alpha$ equal to 0.5 . 


\section{Extended Pompom}

The Pompom model was proposed by McLeish and Larson [97] to describe the strain hardening in elongation and shear thinning behavior in shear for branched polymers. They defined an idealized molecule, the 'pompom', which consists of a backbone with a number of branches at each end. A key feature in this model is the separation of stretch and orientation of a polymer molecule. The original approximative differential model showed discontinuities in steady state elongation and no second normal stress difference, which was shown to influence flow-induced crystallization (Jerschow and Janeschitz-Kriegl [66]). Verbeeten et al. [143] proposed the extended Pompom model, which improved upon these two disadvantages. Excellent quantitative agreement with measurements of branched (LDPE) and linear polymers (HDPE) was found by using a multi mode version. The derivation of the extended Pompom model can be found in Verbeeten et al. [143].

The differential equation of the extended Pompom model is given by

$$
\stackrel{\nabla}{\tau}+\frac{1}{\lambda(\tau)} \cdot \tau=2 G \boldsymbol{D}
$$

with relaxation tensor

$$
\frac{1}{\boldsymbol{\lambda}(\boldsymbol{\tau})}=\frac{1}{\lambda_{0 b}}[\frac{\alpha}{G} \boldsymbol{\tau}+\frac{1}{f(\boldsymbol{\tau})} \boldsymbol{I}+\underbrace{G\left(\frac{1}{f(\boldsymbol{\tau})}-1\right)}_{(a)} \boldsymbol{\tau}^{-1}]
$$

and extra function

$$
\frac{1}{\lambda_{0 b} f(\boldsymbol{\tau})}=\underbrace{\frac{2}{\lambda_{s}}\left(1-\frac{1}{\Lambda}\right)}_{(b)}+\underbrace{\frac{1}{\lambda_{0 b} \Lambda^{2}}\left(1-\frac{\alpha \mathcal{I}_{\boldsymbol{\tau} \cdot \boldsymbol{\tau}}}{3 G^{2}}\right)}_{(c)}
$$

Backbone stretch and stretch relaxation time are defined as

$$
\Lambda=\sqrt{1+\frac{\mathcal{I}_{\tau}}{3 G_{0}}}, \quad \lambda_{s}=\lambda_{0 s} \exp (-\nu(\Lambda-1)), \quad \nu=\frac{2}{q}
$$

in which $q$ is the number of branches. Parameter $\alpha(\alpha \geq 0)$ describes a Giesekus type of anisotropy (Bird et al. [13]). This parameter influences the second normal stress difference only. The Extended Pompom model is equivalent to the original approximative Pompom model for $\alpha=0$. The orientation relaxation times of the backbone $\lambda_{0 b}$ are obtained from linear viscoelastic data. The number of branches $q$, the stretch relaxation times $\lambda_{\mathrm{s}}$ and the anisotropy parameter $\alpha$ have to be determined for each mode. This large number of parameters seems to be a drawback. A physical guideline related to the structure of the Pompom molecule can be taken into account (Verbeeten et al. [143]). The free ends of the molecule correspond to fast relaxation times and no or less branches. Going to the center of the molecule, relaxation and branches will increase. Moreover, the stretch relaxation time is constrained in the interval $\lambda_{0 \mathrm{~b}, \mathrm{i}-1}<\lambda_{0 \mathrm{~s}, \mathrm{i}} \leq \lambda_{0 \mathrm{~b}, \mathrm{i}}$.

Stretch and orientation are gathered in one equation in the extended Pompom model. In case of $\alpha=0$ two situations can be distinguished: 
- Only orientation relaxation for low strains $(\Lambda \approx 1$; part (a) and (b) of Equation (3.19) and (3.20) are equal to zero.)

- Only stretch relaxation for high strains $(\Lambda>>1$; part (c) of Equation (3.20) is equal to zero.)

Peters [112] formulated the interaction of stretch and orientation as follows: 'The orientation knows about the stretch, but the stretch does not know about the orientation'.

\subsection{Constitutive equations for flow-induced nucleation and crystallization}

\subsubsection{Quiescent crystallization}

Non-isothermal crystallization of spherulites can be described by the Schneider's rate equations (Schneider et al. [126, 127]), a set of differential equations, which describe the structure developing in quiescent conditions. Mean number of spherulites and their mean radius, surface and volume are calculated (Equation (3.22-3.25)):

$$
\begin{aligned}
& \dot{\phi}_{3}=8 \pi \alpha \quad\left(\phi_{3}=8 \pi N\right) \quad \text { 'rate' } \\
& \dot{\phi}_{2}=G \phi_{3} \quad\left(\phi_{2}=8 \pi R_{\text {tot }}\right) \quad \text { 'radius' } \\
& \dot{\phi}_{1}=G \phi_{2} \quad\left(\phi_{1}=S_{t o t}\right) \quad \text { 'surface' } \\
& \dot{\phi}_{0}=G \phi_{1} \quad\left(\phi_{0}=V_{t o t}\right) \quad \text { 'volume' } \\
& \phi_{0}=-\ln \left(1-\xi_{g}\right) \quad \text { 'space filling' }
\end{aligned}
$$

with the nucleation rate $\alpha$ and the growth rate $G$. Impingement of the spherulites is captured by an Avrami model (Equation (3.26)). The morphology is described per unit volume by the total volume of spherulites $V_{\text {tot }}$, their total surface $S_{\text {tot }}$, the sum of their radii $R_{\text {tot }}$ and the number of nuclei $N$. The relation of these parameters with $\phi_{i}$ is given between brackets. The number of nuclei and the growth rate have to be measured as a function of temperature.

The often used Avrami equation describes space filling in case of isothermal crystallization, for which all nuclei appear at $t_{0}$ and where the growth rate is constant for $t>t_{0}$. It is given by

$$
\xi_{g}=1-\exp \left[-\frac{4 \pi}{3} N G^{3}\left(t-t_{0}\right)^{3}\right]
$$

with $\mathrm{t}_{0}$ the time that the crystallization temperature was reached. No morphological information is obtained by this equation. Notice that the Avrami equation is a special case of the Schneider rate equations as shown in Eder and Janeschitz-Kriegl [35]. Consequently, predictions of the space filling for Avrami and Schneider are about the same for isothermal crystallization using high cooling rates and low supercooling. The instanteneously created nuclei at $t_{0}$ is observed experimentally as a more or less constant spherulite size after isothermal crystallization. 


\subsubsection{Flow-induced nucleation and crystallization}

Zuidema $[156,157]$ proposed a modification $\left(S_{\mathrm{J} 2}\right.$-model) for the shear-induced crystallization model of Eder and Janeschitz-Kriegl [35], which gave a good description of the flow-induced structures obtained in their experiments. However, this model is based on the shear rate applied and not on molecular dynamics. Consequently, this model is not suitable in (Lagrangian) transient complex flows.

Zuidema used the recoverable strain modeled with a Leonov type of model as a driving force for flow-induced crystallization. It was demonstrated that the flowinduced structure correlated most strongly with the viscoelastic mode with the highest relaxation time. Therefore, only the second invariant of the deviatoric part of the recoverable strain $\left(\mathrm{J}_{2}\left(\overline{\mathbf{B}}_{\mathrm{e}}^{\mathrm{d}}\right)\right)$ for the maximum rheological relaxation time was used. This invariant is a measure for the molecular orientation (Larson [78]).

With the $S_{\mathrm{J} 2}$-model, non-isothermal crystallization of cylindrical structures (which are for convenience called shish-kebabs) can be described. It has the same structure of differential equations as the Schneider rate equations. Mean number of shish-kebabs and their mean length, surface and volume are calculated (Equation (3.28-3.31)):

$$
\begin{aligned}
& \dot{\psi}_{3}+\frac{\psi_{3}}{\tau_{n}}=8 \pi J_{2} g_{n}^{\prime} \quad\left(\psi_{3}=8 \pi N_{f}\right) \quad \text { 'rate' } \\
& \dot{\psi}_{2}+\frac{\psi_{2}}{\tau_{l}}=\psi_{3} J_{2} \frac{g_{l}^{\prime}}{g_{n}^{\prime}} \quad\left(\psi_{2}=4 \pi L_{t o t}\right) \quad \text { 'length' } \\
& \dot{\psi}_{1} \quad=G \psi_{2} \quad\left(\psi_{1}=S_{\text {tot }}\right) \quad \text { 'surface' } \\
& \dot{\psi}_{0} \quad=G \psi_{1} \quad\left(\psi_{0}=V_{t o t}\right) \quad \text { 'volume' } \\
& \psi_{0} \quad=-\ln \left(1-\xi_{g}\right) \quad \text { 'space filling' }
\end{aligned}
$$

with the driving force $\mathrm{J}_{2}$, the growth rate $\mathrm{G}$, the scaling factors $\mathrm{g}_{\mathrm{n}}^{\prime}$ and $\mathrm{g}_{1}^{\prime}$ to describe the sensitivity of the flow-induced nuclei and length on $J_{2}$, and the characteristic times $\tau_{\mathrm{n}}$ and $\tau_{1}$ to describe the relaxation behavior of the flow-induced nuclei and length. Impingement of the cylindrical structures is captured by an Avrami model (Equation (3.32)). The morphology is described per unit volume by the total volume of shish-kebabs $V_{\text {tot }}$, their total surface $S_{\text {tot }}$, the sum of their lengths $L_{\text {tot }}$ and the number of flow-induced nuclei $\mathrm{N}_{\mathrm{f}}$. The relation of these parameters with $\psi_{\mathrm{i}}$ is given between brackets. Eder's rate equations are obtained by replacing $\mathrm{J}_{2}$ by a scaled value of the shear rate squared.

Molecular orientation can generate extra nuclei (Equation (3.28)). When the orientation is strong enough, these nuclei can grow in one direction (Equation (3.29)). The radial growth rate of the cylindrical structures is taken equal to the spherulitical growth rate, because experiments will be performed in which nucleation and growth are separated. As discussed in Section 2.3, growth rate depends weakly on flow after cessation of flow. Of course, other choices for the radial growth dependent on $\mathrm{J}_{2}$ are possible. The relaxation time $\tau_{1}$ is in general taken very large, because reduction of length can only occur via melting. The relaxation time $\tau_{\mathrm{n}}$ was chosen equal to the rheological relaxation time.

Zuidema [156, 157] considered nucleation as physical cross-linking. Consequently, an increased number of nuclei causes an increase in the rheological relaxation time. A 
linear relationship between flow-induced nuclei and the highest rheological relaxation time was chosen.

$$
\theta_{j}=a_{T}(T) \theta_{j 0}\left(1+\frac{\beta N_{f}}{g_{n}^{\prime}}\right)
$$

with $\theta_{\mathrm{j} 0}$ the highest rheological relaxation time at a reference temperature, $\mathrm{a}_{\mathrm{T}}$ the shift factor and $\beta$ a scaling factor that describes the interaction between nuclei and rheology. Consequently, the scaling factors $\beta, g_{n}^{\prime}$ and $g_{l}^{\prime}$ should be measured as a function of flow conditions.

The concept of the equivalence of physical cross-linking and nucleation in the Leonov model can be used in the Pompom model in rather natural way. Increase in the number of nuclei gives an increase in the number of branches and relaxation time. This means that the scaling factor $\beta$ in Equation (3.33) is related to the number of branches in the Pompom model.

In the case of flow, both spherulitical and flow-induced structures contribute to the degree of space filling, depending on the influence of $\mathrm{J}_{2}$. The Avrami model for impingement is then described by

$$
\phi_{0}+\psi_{0}=-\ln \left(1-\xi_{g}\right)
$$

\subsection{Discussion}

Originally, the extended Pompom model was proposed for branced polymers, but it captures also the fysics for linear polymers, as shown by Verbeeten et al. [143] for HDPE. There are two big advantages when applying this model for the linear polymers used in this study. First, both elongation and shear data can be described excellent with the same set of fitting parameters. Second, the physical description that serves as a basis for this model (i.e. the Pompom molecule) results in a transparant model. The physical cross-linking process, proposed by Zuidema in the $S_{\mathrm{J} 2}$ model, can be related to the increase in the number of branches during crystallization.

A general structure was obtained to describe quiescent crystallization (Schneider et al. [126, 127]) and flow-induced crystallization (Eder and Janeschitz-Kriegl [35], Zuidema [156]). The advantage of the use of the $S_{\mathrm{J} 2}$ model rather than the Eder rate equations is the dependence of nucleation rate and growth on a (molecular) strain measure rather than the macroscopic shear rate. This allows the use of the $S_{22}$ model in (Langragian) transient complex flows. Moreover, improvements or other dependencies can be easily inserted (for example a deformation depended growth rate).

The dependence of rheology on nucleation is described by physical cross-links (Leonov) or an enhanced number of branches (Pompom), resulting in an increased relaxation time. The sensitivity of this relation between number of nuclei and rheological relaxation time has to be chosen. 


\section{Chapter 4}

\section{Materials characterization}

\subsection{Introduction}

Experiments were carried out on two commercially isotactic polypropylenes, StamylanP 13E10 and StamylanP 15M10, both provided by DSM, Geleen, the Netherlands. The first material is an extrusion and the second an injection moulding grade. In the following, these materials will be abbreviated as DSM13E10 and DSM15M10.

The main difference between these two materials is the molecular weight (Table 4.1). A small difference in molecular weight distribution and tacticity was found in gel permeation chromatografy (GPC) and Fourier transform infrared spectroscopy (FTIR) respectively (Borealis, Linz, Austria). The GPC data for both materials is shown in Figure 4.1. The two curves are similar in shape, but shifted in molecular weight.

\begin{tabular}{|l||r|r|r|r|}
\hline \multirow{2}{*}{ Material } & \multicolumn{2}{c|}{ GPC } & \multicolumn{1}{c|}{ FTIR } & \multicolumn{1}{c|}{ DSC } \\
\cline { 2 - 5 } & $\mathrm{M}_{\mathrm{w}}[\mathrm{kg} / \mathrm{mol}]$ & $\mathrm{M}_{\mathrm{w}} / \mathrm{M}_{\mathrm{n}}[-]$ & Tacticity [-] & $\mathrm{T}_{\mathrm{m}}\left[{ }^{\circ} \mathrm{C}\right]$ \\
\hline \hline DSM13E10 & 500 & 6.0 & 0.947 & 163.4 \\
\hline DSM15M10 & 350 & 5.6 & 0.962 & 161 \\
\hline
\end{tabular}

Table 4.1: Data for the two polypropylenes used in this study. $\mathrm{M}_{\mathrm{w}}$ : weight average molecular weight, $\mathrm{M}_{\mathrm{n}}$ : number average molecular weight, $\mathrm{M}_{\mathrm{w}} / \mathrm{M}_{\mathrm{n}}$ : molecular weight distribution. Data were provided by Gahleitner/Köningsdorfer (Borealis, Linz, Austria).

Rheological characterization in simple shear and uniaxial elongation are described in Section 4.2, and are used to determine the thermo-rheological behavior, the relaxation time spectrum, and the non-linear parameters for the (viscoelastic) constitutive equations. Microscopy is used to determine nucleation and growth rate in quiescent conditions as a function of temperature. The measurements and fits are shown in Section 4.3. Isothermal crystallization is investigated using DSC (Section 4.4) and rheometry (Section 4.5). The influence of short time shearing at a crystallization temperature of $140\left[{ }^{\circ} \mathrm{C}\right]$ is also investigated, see Section 4.5 . We have attempted to determine the parameters of the $S_{\mathrm{J} 2}$ model for DSM13E10 from these experiments, see Section 4.6. Moreover, the influence on rheology predicted by this model is discussed shortly. 


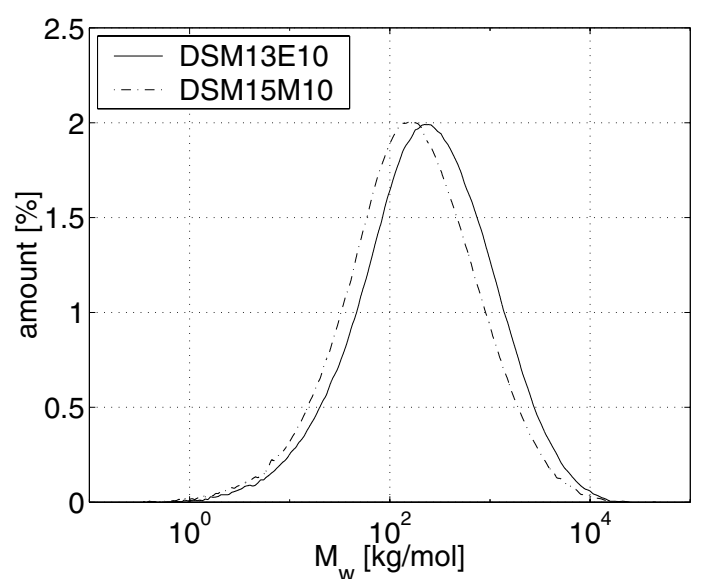

Figure 4.1: Gel permeation chromatografy on DSM13E10 and DSM15M10. Data were provided by Gahleitner/Köningsdorfer (Borealis, Linz, Austria).

\subsection{Rheological characterization}

The melts were characterized in simple shear with a rotational viscometer (Ares 3LS4A, Rheometrics Inc.), using a cone-plate geometry (diameter 25 [mm], cone angle 0.1004 [rad]). All measurements were performed in a nitrogen environment to prevent degradation.

Elongation experiments were performed on an elongational rheometer (Rheometrics RME). The sample supported by a cushion of inert gas (nitrogen) is extended by metal conveyer belts.

Samples for rotational viscometry were made using compression moulding. The circular preforms having a diameter of about $17[\mathrm{~mm}]$ and a thickness of $2[\mathrm{~mm}]$ were moulded at a temperature of $230\left[{ }^{\circ} \mathrm{C}\right]$. Each two minutes the pressing force was increased in seven steps to a maximum value of $50[\mathrm{kN}]$.

The sample preparation for the RME was different in order to obtain stress free, homogeneous samples (with dimensions $55 \times 7 \times 1.5\left[\mathrm{~mm}^{3}\right]$ ). The polymer pellets were first grinded, and compression moulding and cooling of the samples were performed more gently.

Oscillatory shear measurements were performed in a frequency range of 0.1-100 [Hz] at temperatures between 160 and $220\left[{ }^{\circ} \mathrm{C}\right], 0.01-100[\mathrm{~Hz}]$ at $240\left[{ }^{\circ} \mathrm{C}\right]$ and $1-250$ $[\mathrm{Hz}]$ at $140\left[{ }^{\circ} \mathrm{C}\right]$. The range was extended to lower frequencies at $240\left[{ }^{\circ} \mathrm{C}\right]$, but was constrained by the lower limit of the transducer. Only the high frequencies were measured at $140\left[{ }^{\circ} \mathrm{C}\right]$ to limit the total measuring time at this temperature. Samples were annealed at the measuring temperature for 15 minutes, except for the measurements below the melting temperature (140 and $\left.160\left[{ }^{\circ} \mathrm{C}\right]\right)$, for which the samples were annealed at $180\left[{ }^{\circ} \mathrm{C}\right]$. For the measurement at $140\left[{ }^{\circ} \mathrm{C}\right]$, liquid nitrogen was used to cool the device within $95[\mathrm{~s}]$ from the annealing temperature $\left(180\left[{ }^{\circ} \mathrm{C}\right]\right)$. This measurement was started 300 [s] after cooling the sample. In Section 4.5, it will be shown that the modulus is not influenced by crystallization within 1000 [s] at $140\left[{ }^{\circ} \mathrm{C}\right]$.

Steady shear measurements were only performed in the interval 0.01-1 [s $\left.\mathrm{s}^{-1}\right]$ at temperatures between 195 and $240\left[{ }^{\circ} \mathrm{C}\right]$. For higher shear rates edge failure occurred. Edge 
failure is caused by the elastic properties of polymeric materials. This instability starts at the edge of the sample as a crack that grows to the center. It is more pronounced in cone-plate than in plate-plate set-ups. Edge failure can be observed during the measurement as a decreasing viscosity and first normal stress difference in time. Gleißle [53] and Pahl et al. [109] have proposed an extra ring around the cone-plate geometry to decrease stresses at the surface and therefore reduce edge failure.

Steady and dynamic measurements should fulfill the empirical Cox-Merz rule for these materials. This rule states that the magnitude of the dynamic viscosity is equal to the steady viscosity at corresponding values of frequency and shear rate

$$
\eta(\dot{\gamma})=\left.\eta_{d}(\omega)\right|_{\omega=\dot{\gamma}}
$$

Rheological characterization of the two materials consisted of the following steps:

1. Determination of the dynamic shift factors.

2. Determination of the parameters for the thermo-rheological equations.

3. Determination of the relaxation time spectrum.

4. Determination of the (non-linear) parameters for the constitutive equations.

\section{ad 1. Determination of the dynamic shift factors}

Master curves were created at a reference temperature of $220\left[{ }^{\circ} \mathrm{C}\right]$ using time-temperature superposition. The horizontal shift factor $a_{T}$ and the vertical shift factor $b_{\mathrm{T}}$ were determined independently, by shifting the loss angle $\delta\left(\mathrm{a}_{\mathrm{T}}\right)$ and dynamic modulus $\mathrm{G}_{\mathrm{d}}$ $\left(b_{T}\right)$ onto a single master curve. The fitted shift factors determined from the dynamic experiments, were used to shift also the steady curves to the reference temperature (Table 4.2). The shift factor $b_{\mathrm{T}}$, defined as

$$
b_{T}=\frac{T_{r e f} \rho_{r e f}}{T \rho}
$$

describes the dependence of stresses on temperature and density and is very often taken equal to one. However, here it is necessary to use this factor, because the difference of the modulus with the reference modulus can be more than $10 \%$ in the large temperature range considered.

\section{ad 2. Determination of the parameters for the thermo-rheological equations}

The thermo-mechanical behavior of the materials was fitted by the Arrhenius, and the Williams, Landel and Ferry (WLF) equation. The Arrhenius equation is given by

$$
\ln \left(a_{T}\right)=\frac{E_{r e f}}{R}\left(\frac{1}{T}-\frac{1}{T_{r e f}}\right)
$$

with $\mathrm{E}_{\text {ref }}$ the material dependent flow activation energy and $\mathrm{R}$ the Boltzmann gas constant $(\mathrm{R}=8.314[\mathrm{~J} /(\mathrm{mol} \mathrm{K})])$. The WLF equation is given by

$$
\log \left(a_{T}\right)=-\frac{c_{1}\left(T-T_{r e f}\right)}{c_{2}+\left(T-T_{r e f}\right)}
$$




\begin{tabular}{|l||l|l||l|}
\hline Flow & Name & Symbol & Master curves \\
\hline \hline \multirow{4}{*}{$\begin{array}{l}\text { Small amplitude } \\
\text { oscillatory shear }\end{array}$} & storage modulus & $\mathrm{G}^{\prime}[\mathrm{Pa}]$ & $\mathrm{G}^{\prime}\left(\omega \mathrm{a}_{\mathrm{T}}, \mathrm{T}_{\mathrm{ref}}\right)=\mathrm{G}^{\prime}(\omega, \mathrm{T}) \mathrm{b}_{\mathrm{T}}$ \\
\cline { 2 - 5 } & loss modulus & $\mathrm{G}^{\prime \prime}[\mathrm{Pa}]$ & $\mathrm{G}^{\prime \prime}\left(\omega \mathrm{a}_{\mathrm{T}}, \mathrm{T}_{\mathrm{ref}}\right)=\mathrm{G}^{\prime \prime}(\omega, \mathrm{T}) \mathrm{b}_{\mathrm{T}}$ \\
\cline { 2 - 5 } & dynamic modulus & $\mathrm{G}_{\mathrm{d}}[\mathrm{Pa}]$ & $\mathrm{G}_{\mathrm{d}}\left(\omega \mathrm{a}_{\mathrm{T}}, \mathrm{T}_{\mathrm{ref}}\right)=\mathrm{G}_{\mathrm{d}}(\omega, \mathrm{T}) \mathrm{b}_{\mathrm{T}}$ \\
\cline { 2 - 5 } & loss angle & $\delta[-]$ & $\delta\left(\omega \mathrm{a}_{\mathrm{T}}, \mathrm{T}_{\mathrm{ref}}\right)=\delta(\omega, \mathrm{T})$ \\
\hline \hline \multirow{3}{*}{ Steady shear } & viscosity & $\eta[\mathrm{Pa} . \mathrm{s}]$ & $\eta\left(\dot{\gamma} \mathrm{a}_{\mathrm{T}}, \mathrm{T}_{\mathrm{ref}}\right)=\eta(\dot{\gamma}, \mathrm{T}) \mathrm{b}_{\mathrm{T}} / \mathrm{a}_{\mathrm{T}}$ \\
\cline { 2 - 5 } & $\begin{array}{l}\text { first normal stress } \\
\text { difference }\end{array}$ & $\mathrm{N}_{1}[\mathrm{~Pa}]$ & $\mathrm{N}_{1}\left(\dot{\gamma} \mathrm{a}_{\mathrm{T}}, \mathrm{T}_{\mathrm{ref}}\right)=\mathrm{N}_{1}(\dot{\gamma}, \mathrm{T}) \mathrm{b}_{\mathrm{T}}$ \\
\cline { 2 - 4 } & first normal stress & $\Psi_{1}\left[\mathrm{~Pa} . \mathrm{s}^{2}\right]$ & $\Psi_{1}\left(\dot{\gamma} \mathrm{a}_{\mathrm{T}}, \mathrm{T}_{\mathrm{ref}}\right)=\Psi_{1}(\dot{\gamma}, \mathrm{T}) \mathrm{b}_{\mathrm{T}} / \mathrm{a}_{\mathrm{T}}^{2}$ \\
\hline
\end{tabular}

Table 4.2: Material functions in simple-shear flows (Ferry [38]).

with $c_{1}$ and $c_{2}$ the WLF constants. The constants of these two equations were estimated with least squares fits.

\section{ad 3. Determination of the relaxation time spectrum}

A multi-mode Maxwell model describes accurately the linear viscoelastic behavior. Using this model, the dynamic moduli are given by

$$
\begin{aligned}
G^{\prime} & =\sum_{i=1}^{N} \frac{G_{i} \lambda_{i}^{2} \omega^{2}}{1+\lambda_{i}^{2} \omega^{2}} \\
G^{\prime \prime} & =\sum_{i=1}^{N} \frac{G_{i} \lambda_{i} \omega}{1+\lambda_{i}^{2} \omega^{2}}
\end{aligned}
$$

The number of modes $\mathrm{N}$ depends on the frequency range and should be 1.2-1.5 modes per decade (Baumgaertel and Winter [10]). The Maxwell parameters $\left(G_{i}, \lambda_{i}\right)$ were determined by fitting the Maxwell model on the dynamic moduli with a LevenberghMarquardt algorithm (Zoetelief [155]). These individual discrete relaxation times have no physical meaning. All modes together represent the continuous relaxation behavior of the material.

A characteristic time for the material was defined as a viscosity averaged value for the relaxation time of the fluid ( $\bar{\lambda}$; Schoonen [128], Schoonen et al. [129]).

$$
\bar{\lambda}=\frac{\sum_{i=1}^{N} \eta_{i} \lambda_{i}}{\sum_{i=1}^{N} \eta_{i}}
$$




\section{ad 4. Determination of the parameters for the constitutive equations}

One generalized Newtonian (Carreau-Yasuda) and two non-linear viscoelastic constitutive equations (Extended Pompom and Leonov) were fitted on the rheological data. For both the non-linear viscoelastic equations the multi-mode form was used.

Carreau-Yasuda only describes the viscosity. It was chosen to fit the model only on the accurate dynamic measurements. This is allowed if the empirical Cox-Merz rule is fulfilled, which was therefore checked first.

The Pompom model, which is known to give an excellent description of the material in both shear as elongation, Verbeeten et al. [143, 144], was fitted manually on the uniaxial elongational data only. Two parameters can be varied for each mode, the number of branches $\mathrm{q}$ and the relaxation ratio $\lambda_{0 \mathrm{~b}} / \lambda_{0 \mathrm{~s}}{ }^{*}$. The Leonov model does not have any non-linear fitting parameters and its performance is completely determined by the linear viscoelastic data.

The measurements and fits are presented in the next two subsections for the high molecular weight (DSM13E10) and the low molecular weight material (DSM15M10), respectively.

\subsubsection{High molecular weight (DSM13E10)}

The results for DSM13E10 are shown in Figures 4.2-4.7. The parameters for the fitted equations are given in Table 4.3. Figure 4.2 shows that the WLF equation gives the best description of the shiftfactor $\mathrm{a}_{\mathrm{T}}$. The shift factor $\mathrm{b}_{\mathrm{T}}$ is less pronounced and close to one (Table 4.3). The dynamic master curves were fitted with an eight mode Maxwell model in Figure 4.3(b). The characteristic time for this material of about 16 [s] at $220\left[{ }^{\circ} \mathrm{C}\right]$ is rather high.

The steady master curves are given in Figure 4.4. The measurement at $195\left[{ }^{\circ} \mathrm{C}\right]$ was performed by DSM Research using a capillary rheometer. The steady viscosities are slightly higher than the dynamic viscosities. This discrepancy becomes less by using preshearing of the sample. It was concluded that Cox-Merz is valid. The fitted zeroshear viscosity for the Carreau-Yasuda model is slightly higher than for the Maxwell model, because the zero viscosity region was not reached in the dynamic experiments. Viscosity and first normal stress coefficient are described well by the constitutive equations in steady shear.

The transient behavior during startup of shear and after cessation of shear are shown in Figure 4.5-4.6. The measured first normal stresses were too weak for these low shear rates at the measurement temperature of $200\left[{ }^{\circ} \mathrm{C}\right]$ and are therefore not presented. Startup of shear is described equally well by the viscoelastic equations. Pompom seems to give a better description for cessation of shear, but a small deviation is found at $0.145\left[\mathrm{~s}^{-1}\right]$.

The transient and steady uniaxial elongation behavior are shown in Figure 4.7. Measurements were performed by DSM Research at a temperature of $172\left[{ }^{\circ} \mathrm{C}\right]$. The steady uniaxial elongational viscosity shows mild elongational thickening followed by elongational thinning. The Pompom model gives a reasonable fit, while the Leonov

\footnotetext{
*The second normal stress difference is needed to fit parameter $\alpha$. Since this data is not available, $\alpha$ is taken equal to $0.1 / \mathrm{q}$ (Verbeeten et al. [143])
} 
model cannot describe these measurements. The number of arms or equivalent 'branches' $\mathrm{q}$ in the Pompom model is low, as could be expected for this linear polymer.

\subsubsection{Low molecular weight (DSM15M10)}

The results for DSM15M10 are shown in Figures 4.8-4.11. The parameters for the fitted equations are given in Table 4.4. Again, the WLF equation gives a better fit for the $\mathrm{a}_{\mathrm{T}}$ shift factor. The dynamic master curves were fitted with a seven mode Maxwell model as shown in Figure 4.9(b). The characteristic time for this material is about 1.4 [s] at $220\left[{ }^{\circ} \mathrm{C}\right]$ and is one order of magnitude lower than for DSM13E10.

The steady master curves are given in Figure 4.10. Again, the steady viscosities are slightly higher than the dynamic viscosity, but the Cox-Merz rule is valid. The fitted zero-shear viscosity for the Carreau-Yasuda model is about the same as for the Maxwell model. Viscosity and first normal stress coefficient are described well by the constitutive equations in steady shear.

The transient and steady uniaxial elongation behavior are shown in Figure 4.11. It is quite difficult to perform an uniaxial elongation experiment on this low molecular weight material. Therefore, only one experiment, performed by DSM Research at $189\left[{ }^{\circ} \mathrm{C}\right]$, is shown. Other experimental results show strain hardening behavior, but this is due to an inhomogeneous deformation of the sample and cannot be regarded as a material property (See Hepperle and Saito [59] for an example on iPP). The transient data is fitted well by both Pompom and Leonov. The steady state elongational viscosity (Figure 4.11(b)) shows that this is a coincidence, because the intrinsic behavior of both models is different. In comparison to DSM13E10, the number of branches is chosen slightly lower to fit the steady state elongational viscosity at $2.08\left[\mathrm{~s}^{-1}\right]$.

\subsection{Determination of $\mathrm{G}(\mathrm{T})$ and $\mathrm{N}(\mathrm{T})$}

Measurements were performed with a combined hot-stage (Linkam, TP93)-microscopy (Zeiss, Axiolab) set-up. The hot-stage consists of a electrically controlled heating-plate, which has a hole of about 2.6 [mm] in diameter to perform transmission microscopy. The plate can be cooled with liquid nitrogen using a pump (Linkam, LNP1). The microscope was connected with a CCD video camera (Sony, DXC-151 AP) and a time-lapsed video recorder (Panasonic), necessary to tape the experiments at the crystallization temperatures up to $148\left[{ }^{\circ} \mathrm{C}\right]$, which can last more than one day. Details of the set-up are described in Appendix A. Temperature was calibrated with liquid crystalline polymers as described in Appendix B.

A small part was cut from a granulate piece (about 5 [mg]) and was positioned between two cover glasses having a thickness of about $0.146[\mathrm{~mm}]$, each. The sample was melted on a hot-plate and pressed by a weight of $5[\mathrm{~kg}]$ to reduce the sample thickness to 30-40 $[\mu \mathrm{m}]$. Next, the sample was inserted in the hot-stage and annealed for 1520 [min] at a temperature of about $240\left[{ }^{\circ} \mathrm{C}\right]$. Then, the sample was cooled fast to the preferred crystallization temperature $\mathrm{T}_{\mathrm{c}}$. The maximum cooling rate of the device was specified as $30\left[{ }^{\circ} \mathrm{C} / \mathrm{min}\right]$. However, a cooling rate of about $80\left[{ }^{\circ} \mathrm{C} / \mathrm{min}\right]$ was obtained 


\begin{tabular}{|c|c|c|c|c|}
\hline \multirow{7}{*}{ Shift factors } & $\overline{\mathrm{T}\left[{ }^{\circ} \mathrm{C}\right]}$ & \multicolumn{2}{|c|}{$\overline{\mathrm{a}_{\mathrm{T}}[-]}$} & 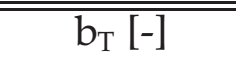 \\
\hline & 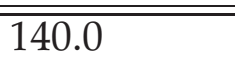 & \multicolumn{2}{|c|}{7.010} & 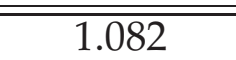 \\
\hline & 160.0 & \multicolumn{2}{|c|}{4.048} & 1.041 \\
\hline & 180.0 & \multicolumn{2}{|c|}{2.174} & 1.008 \\
\hline & 200.0 & \multicolumn{2}{|c|}{1.428} & 0.992 \\
\hline & 220.0 & \multicolumn{2}{|c|}{1.000} & 1.000 \\
\hline & 240.0 & \multicolumn{2}{|c|}{0.733} & 0.983 \\
\hline \multirow{3}{*}{ WLF } & & \multicolumn{2}{|c|}{ 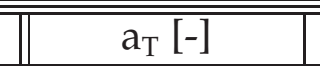 } & $\overline{\mathrm{b}_{\mathrm{T}}[-]}$ \\
\hline & $\mathrm{c}_{1}[-]$ & \multicolumn{2}{|c|}{2.018} & $1.121 \cdot 10^{-2}$ \\
\hline & $\mathrm{C}_{2}[\mathrm{~K}]$ & \multicolumn{2}{|c|}{$2.686 \cdot 10^{2}$} & $1.058 \cdot 10^{2}$ \\
\hline \multirow{2}{*}{ Arrhenius } & & \multicolumn{2}{|c|}{ 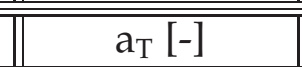 } & 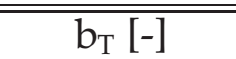 \\
\hline & $\overline{\mathrm{E}_{\mathrm{ref}}[\mathrm{kJ} / \mathrm{mol}]}$ & \multicolumn{2}{|c|}{40.30} & 1.324 \\
\hline \multirow{11}{*}{ Maxwell } & mode & \multicolumn{2}{|c|}{ 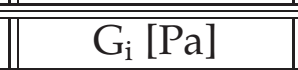 } & 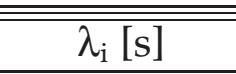 \\
\hline & 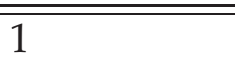 & \multicolumn{2}{|c|}{$9.859 \cdot 10^{4}$} & $6.769 \cdot 10^{-4}$ \\
\hline & 2 & \multicolumn{2}{|c|}{$5.615 \cdot 10^{4}$} & $4.478 \cdot 10^{-3}$ \\
\hline & 3 & \multirow{2}{*}{\multicolumn{2}{|c|}{$\begin{array}{l}3.395 \cdot 10^{4} \\
1.613 \cdot 10^{4}\end{array}$}} & $2.265 \cdot 10^{-2}$ \\
\hline & 4 & & & $1.090 \cdot 10^{-1}$ \\
\hline & 5 & \multicolumn{2}{|c|}{$5.313 \cdot 10^{3}$} & $5.205 \cdot 10^{-1}$ \\
\hline & 6 & \multirow{2}{*}{\multicolumn{2}{|c|}{$\begin{array}{l}1.209 \cdot 10^{3} \\
1.917 \cdot 10^{2}\end{array}$}} & $2.502 \cdot 10^{0}$ \\
\hline & 7 & & & $1.344 \cdot 10^{1}$ \\
\hline & 8 & \multicolumn{2}{|c|}{$1.700 \cdot 10^{1}$} & $9.732 \cdot 10^{1}$ \\
\hline & & \multicolumn{2}{|c|}{$\eta_{\text {tot }}[\mathrm{Pa} \mathrm{s}]$} & $\overline{\bar{\lambda}}[\mathrm{s}]$ \\
\hline & & \multicolumn{2}{|c|}{$1.287 \cdot 10^{4}$} & $1.592 \cdot 10^{1}$ \\
\hline \multirow{2}{*}{ Carreau-Yasuda } & $\eta_{0}[\mathrm{~Pa} \mathrm{~s}]$ & \multicolumn{2}{|l|}{$\overline{\overline{\lambda_{\mathrm{cy}}[\mathrm{s}]}}$} & a [ [-] \\
\hline & $1.522 \cdot 10^{4}$ & \multicolumn{2}{|l|}{0.1309} & 0.3669 \\
\hline \multirow{9}{*}{ Pompom } & mode & \multicolumn{2}{|c|}{$\mathrm{q}_{\mathrm{i}}[-]$} & 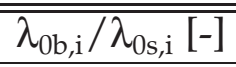 \\
\hline & $\overline{1}$ & & & 2 \\
\hline & 2 & & & 2 \\
\hline & 3 & & & 2 \\
\hline & 4 & & & 2 \\
\hline & 5 & & & 2 \\
\hline & 6 & & & 2 \\
\hline & 7 & & & 1 \\
\hline & 8 & & & 1 \\
\hline
\end{tabular}

Table 4.3: Parameters of the fits for the WLF and Arrhenius equations, and the Maxwell, the Carreau-Yasuda and the Pompom model in case of DSM13E10 $\left(\mathrm{T}_{\text {ref }}=220\left[{ }^{\circ} \mathrm{C}\right]\right) \cdot \eta_{\text {tot }}$ : summation of $\eta_{i}\left(=G_{i} \cdot \lambda_{i}\right), \bar{\lambda}$ : viscosity averaged value for the relaxation time, q: number of branches, $\lambda_{0 \mathrm{~b}} / \lambda_{0 \mathrm{~s}}$ : ratio of the backbone relaxation to the stretch relaxation. 


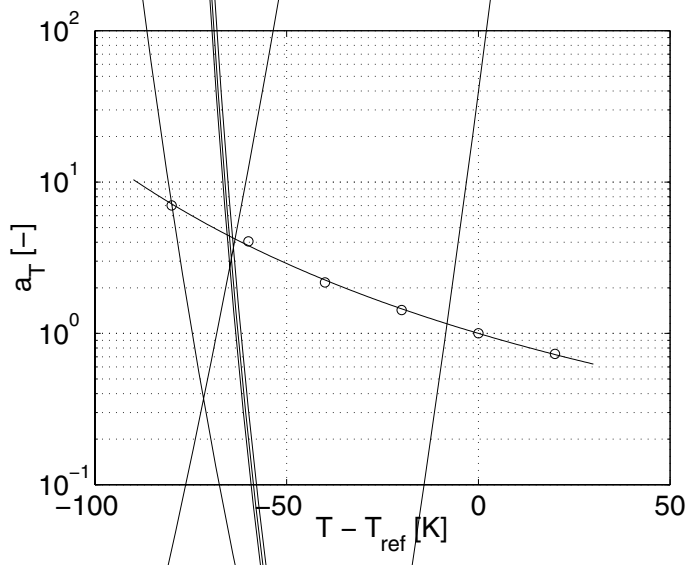

(a)

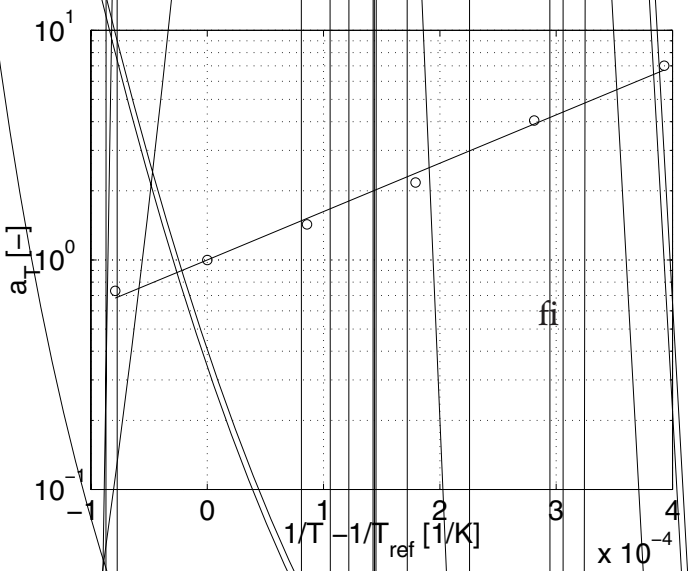

(b)

Figure 4.2: (a) WLF and (b) Arrhenius fit of the shift factors $\mathrm{a}_{\mathrm{T}}$ for DSM13E10 at $\mathrm{T}_{\text {ref }}=220\left[{ }^{\circ} \mathrm{C}\right]$.

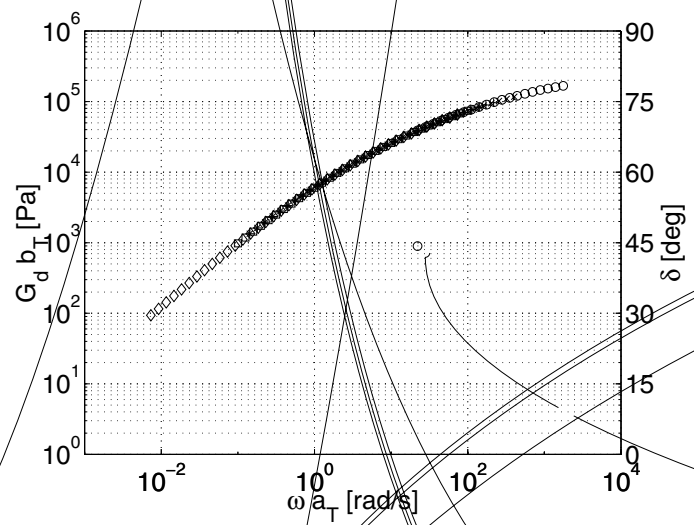



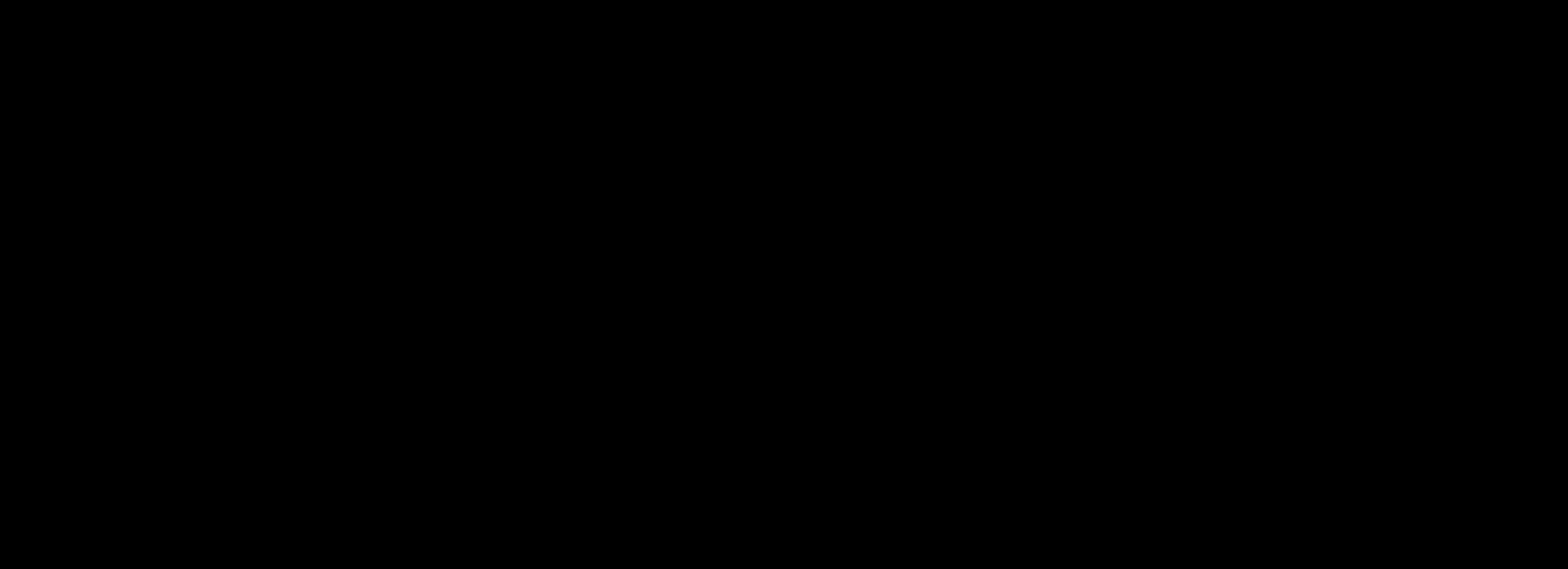

(a) 


\begin{tabular}{|c|c|c|c|c|}
\hline \multirow{7}{*}{ Shift factors } & $\overline{\mathrm{T}\left[{ }^{\circ} \mathrm{C}\right]}$ & \multicolumn{2}{|c|}{$\overline{\mathrm{a}_{\mathrm{T}}[-]}$} & $\overline{\mathrm{b}_{\mathrm{T}}[-]}$ \\
\hline & 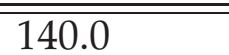 & \multicolumn{2}{|c|}{8.790} & $\overline{c 1.029}$ \\
\hline & 160.0 & \multicolumn{2}{|c|}{4.397} & 1.043 \\
\hline & 180.0 & \multicolumn{2}{|c|}{2.804} & 1.024 \\
\hline & 200.0 & \multirow{2}{*}{\multicolumn{2}{|c|}{1.666}} & 1.020 \\
\hline & 220.0 & & & 1.000 \\
\hline & 240.0 & \multicolumn{2}{|c|}{0.557} & 0.953 \\
\hline \multirow{3}{*}{ WLF } & & \multicolumn{2}{|c|}{$\overline{\mathrm{a}_{\mathrm{T}}[-]}$} & $\overline{\mathrm{b}_{\mathrm{T}}[-]}$ \\
\hline & $\bar{c} c_{1}[-]$ & \multicolumn{2}{|c|}{$3.512 \cdot 10^{1}$} & - \\
\hline & $\mathrm{c}_{2}[\mathrm{~K}]$ & \multicolumn{2}{|c|}{$3.140 \cdot 10^{3}$} & - \\
\hline \multirow{2}{*}{ Arrhenius } & & \multicolumn{2}{|c|}{ 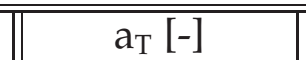 } & 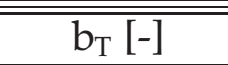 \\
\hline & $\mathrm{E}_{\mathrm{ref}}[\mathrm{kJ} / \mathrm{mol}$ & \multicolumn{2}{|c|}{$\overline{46.04}$} & $\overline{0.979}$ \\
\hline \multirow{10}{*}{ Maxwell } & $\begin{array}{l}\text { mode } \\
\end{array}$ & \multicolumn{2}{|c|}{$\bar{~} \mathrm{G}_{\mathrm{i}}[\mathrm{Pa}]$} & 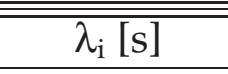 \\
\hline & $\overline{11}$ & \multicolumn{2}{|c|}{$9.689 \cdot 10^{4}$} & \multirow{2}{*}{$\begin{array}{l}5.606 \cdot 10^{-4} \\
3.690 \cdot 10^{-3}\end{array}$} \\
\hline & 2 & \multicolumn{2}{|c|}{$5.096 \cdot 10^{4}$} & \\
\hline & 3 & \multicolumn{2}{|c|}{$2.576 \cdot 10^{4}$} & $1.868 \cdot 10^{-2}$ \\
\hline & 4 & \multicolumn{2}{|c|}{$9.805 \cdot 10^{3}$} & \multirow{2}{*}{$8.869 \cdot 10^{-2}$} \\
\hline & 5 & \multicolumn{2}{|c|}{$2.331 \cdot 10^{3}$} & \\
\hline & 6 & \multicolumn{2}{|c|}{$3.111 \cdot 10^{2}$} & \multirow{2}{*}{$\begin{array}{l}2.272 \cdot 10^{0} \\
1.288 \cdot 10^{1}\end{array}$} \\
\hline & 7 & \multicolumn{2}{|c|}{$1.655 \cdot 10^{1}$} & \\
\hline & & \multicolumn{2}{|c|}{$\eta_{\text {tot }}[$ Pa s $]$} & $\bar{\lambda}[\mathrm{s}]$ \\
\hline & & \multicolumn{2}{|c|}{$3.516 \cdot 10^{3}$} & $1.385 \cdot 10^{0}$ \\
\hline Couman Youd & 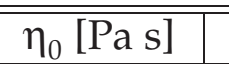 & 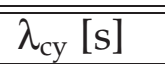 & "n [-] & $\overline{~ a ~[-]}$ \\
\hline Carreau-rasuá & $3.877 \cdot 10^{3}$ & $\overline{0.06570}$ & 0.2192 & 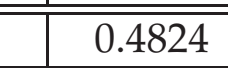 \\
\hline & $\begin{array}{l}\text { mode } \\
\end{array}$ & & & 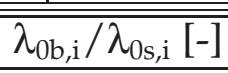 \\
\hline & $\bar{~} 1$ & & & $\overline{2}$ \\
\hline & 2 & & & 2 \\
\hline Pompom & 3 & & & 2 \\
\hline & 4 & & & 2 \\
\hline & 5 & & & 2 \\
\hline & 6 & & & 1 \\
\hline & 7 & & & 1 \\
\hline
\end{tabular}

Table 4.4: Parameters of the fits for the WLF and Arrhenius equations, and the Maxwell, the Carreau-Yasuda and the Pompom model in case of DSM15M10 $\left(\mathrm{T}_{\text {ref }}=220\left[{ }^{\circ} \mathrm{C}\right]\right) \cdot \eta_{\text {tot }}$ : summation of $\eta_{i}\left(=G_{i} \cdot \lambda_{i}\right), \bar{\lambda}$ : viscosity averaged value for the relaxation time, $q:$ number of branches, $\lambda_{0 \mathrm{~b}} / \lambda_{0 \mathrm{~s}}$ : ratio of the backbone relaxation to the stretch relaxation. 


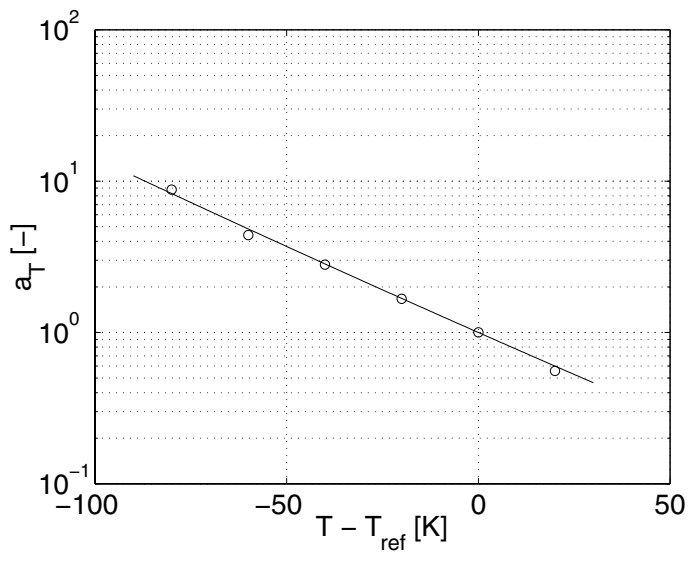

(a)

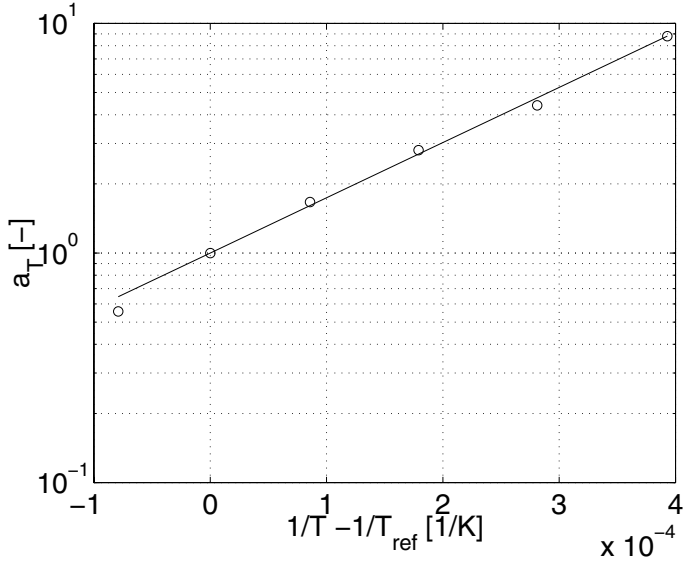

(b)

Figure 4.8: (a) WLF and (b) Arrhenius fit of the shift factors $\mathrm{a}_{\mathrm{T}}$ for DSM15M10 at $\mathrm{T}_{\mathrm{ref}}=220\left[{ }^{\circ} \mathrm{C}\right]$.

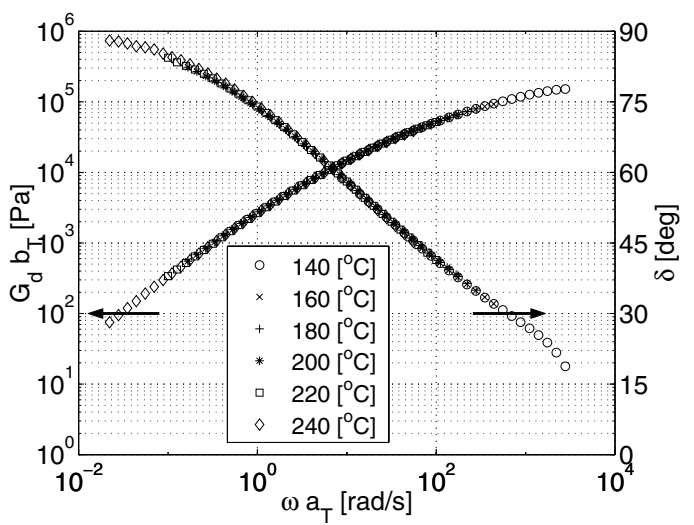

(a)

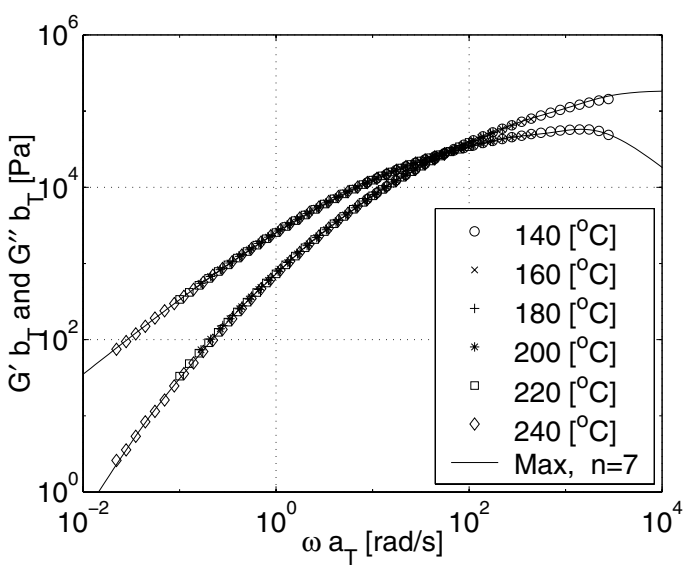

(b)

Figure 4.9: (a) Dynamic modulus and loss angle and (b) the loss and storage modulus with a seven mode Maxwell fit for DSM15M10 at $\mathrm{T}_{\text {ref }}=220\left[{ }^{\circ} \mathrm{C}\right]$.

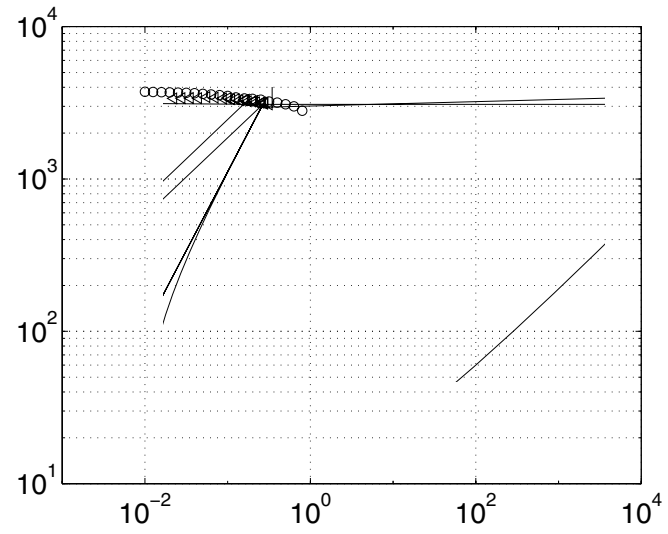




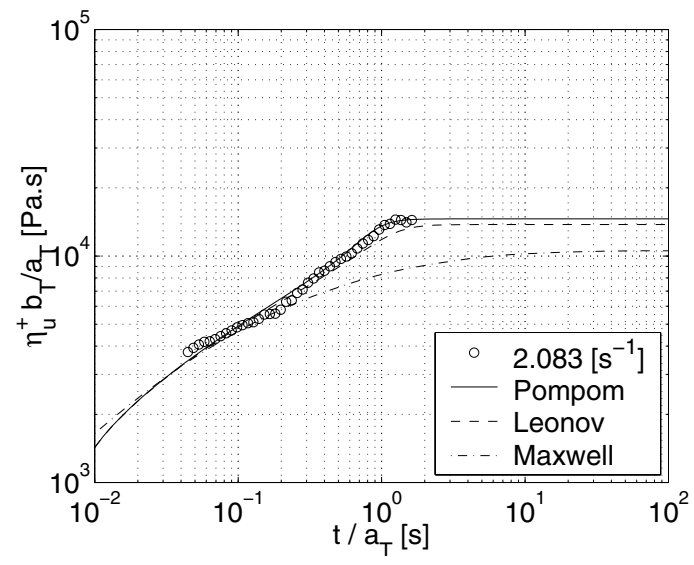

(a)

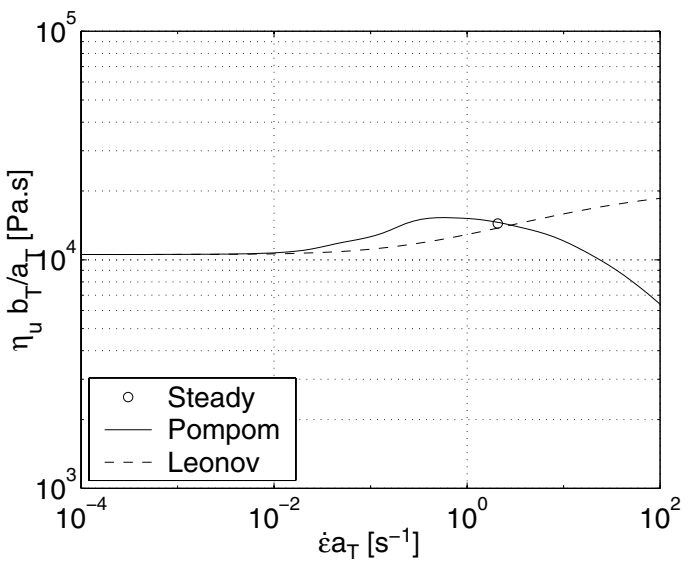

(b)

Figure 4.11: (a) Transient and (b) steady state uniaxial elongational viscosity with the Pompom fit and the Leonov prediction for DSM15M10 $\left(\mathrm{T}_{\text {ref }}=220\left[{ }^{\circ} \mathrm{C}\right]\right)$. The measurement was performed by DSM Research, Geleen, the Netherlands at $189\left[{ }^{\circ} \mathrm{C}\right]$ and shifted to $\mathrm{T}_{\text {ref }}\left(220\left[{ }^{\circ} \mathrm{C}\right]\right)$. The Maxwell equation gives the linear viscoelastic behavior.

by switching off the heating and by using the maximum setting of the liquid nitrogen pump. The heating was switched on at $4\left[{ }^{\circ} \mathrm{C}\right]$ above $\mathrm{T}_{\mathrm{c}}$, and the cooling was reduced at $2\left[{ }^{\circ} \mathrm{C}\right]$ and switched off at $0.4\left[{ }^{\circ} \mathrm{C}\right]$ above $\mathrm{T}_{\mathrm{c}}$ in order to prevent an undershoot in temperature. Finally, isothermal crystallization was monitored.

The determination of $\mathrm{G}(\mathrm{T})$ and $\mathrm{N}(\mathrm{T})$ are described in more detail in the next two subsections. Experimental methods to determine growth rates down to $82\left[{ }^{\circ} \mathrm{C}\right]$ were described by Ratajski and Janeschitz-Kriegl [118].

\subsubsection{Determination of $\mathrm{G}(\mathrm{T})$}

Growth rate experiments were performed in a temperature range of $124-148\left[{ }^{\circ} \mathrm{C}\right]$. At lower temperatures, the experiment was limited by the time and camera resolution, because both growth rate and number of nuclei become very large at these temperatures. Moreover, the thermal conditions after cooling could not have reached a stationary situation before growth sets in.

The determination of the growth rate consisted of three steps. First, the video images were captured and digitized using Adobe Premiere. About 8-10 images were saved starting just after the observation till impingement of spherulites. Second, the images were loaded in Matlab (The MathWorks, Inc) and the edge of the spherulite was marked manually. These points were fitted with circles to determine the radius of the spherulite. Third, these radii as a function of time were fitted with a linear function. The pixelsize was determined with a transmission micrometer. Calibration values for different objectives are given in Table A.5. A long distance $20 \times$ objective was used in all growth experiments.

An example of the procedure is given in Figure 4.12 for a DSM15M10 sample. A two dimensional spherulite with a well defined Maltese cross is shown in Figure 4.12(a). The measured spherulite edges and the fitted circles are shown in Figure 4.12(b). The linear fit of the radius in time is given in Figure 4.12(c). Time $t=0$ [s] was defined as the 
moment after cooling the sample and reaching the crystallization temperature. For this example, the fit gives a different starting moment for nucleation than the measurement. This slight deviation could be explained by the temperature gradient in the sample, which is described in Appendix B. The investigated spherulite is quite large and will be influenced by a gradient of about $0.3\left[{ }^{\circ} \mathrm{C}\right]$ within the region of this image. The measurement in Figure 4.12(c) shows a tendency to grow, initially, slightly faster than at the end. It can be seen, and it is well known, that the growth rate is very sensitive to temperature.

Growth rate can be described by a quadratic exponential function of the temperature between the glass transition temperature and the melting temperature. Since measurements were only performed in a relatively small range below the melting temperature, a linear exponential function was chosen:

$$
G(T)=G_{r e f} \exp \left\{-c_{G}\left(T-T_{\text {cref }}\right)\right\}
$$

with $G_{\text {ref }}$ the growth rate at the crystallization reference temperature $T_{\text {cref }}$ and $c_{G} a$ constant. These two constants were estimated with a least squares fit on the mean growth rates of the investigated temperatures, because the number of points is not equal for each temperature.

The determined growth rates as a function of temperature, together with their fits, are shown in Figure 4.13. The growth rate of DSM13E10 is slightly lower than that of DSM15M10. Considering the available literature, the difference in molecular weight should not be a reason for the difference in growth rate in case of iPP, but is attributed to the (small) difference in tacticity (as discussed in Section 2.3, see Table 4.1).

\subsubsection{Determination of $\mathrm{N}(\mathrm{T})$}

Nucleation experiments were performed in a temperature range of $120-148\left[{ }^{\circ} \mathrm{C}\right]$. Due to the large temperature gradient in the growth rate samples, a different strategy had to be used to get uniform nucleation conditions in the sample. Five granulate pieces were put between two cover glasses ordered like number five on a die. The center piece was used to follow the crystallization conditions with the microscope, while the outer pieces were used to determine the number of nuclei or the number of spherulites. The distance between the outer and the center spot was about $6-8[\mathrm{~mm}]$. Thermal gradients at the four outer pieces should be smaller than at the center due to the direct contact of the glass with the hot-stage. The number of nuclei were counted, divided by the area of the image and converted into nuclei per cubic meter (Eder and Janeschitz-Kriegl [35], Gahleitner et al. [46])

$$
N=(\text { number of nuclei/area })^{\frac{3}{2}}
$$

The size of the spherulites was used as a measure for the temperature at the outer four spots. The largest spherulites in these points were used to determine the local temperature by using Equation (4.8). The result for such a calibration for DSM15M10 is given in Table 4.5. It can be concluded that it is difficult to get a proper heat transfer between the hot-stage and the sample. The deviation in temperature between the points is about $1\left[{ }^{\circ} \mathrm{C}\right]$. The difference between the hot-stage temperature and the mean 


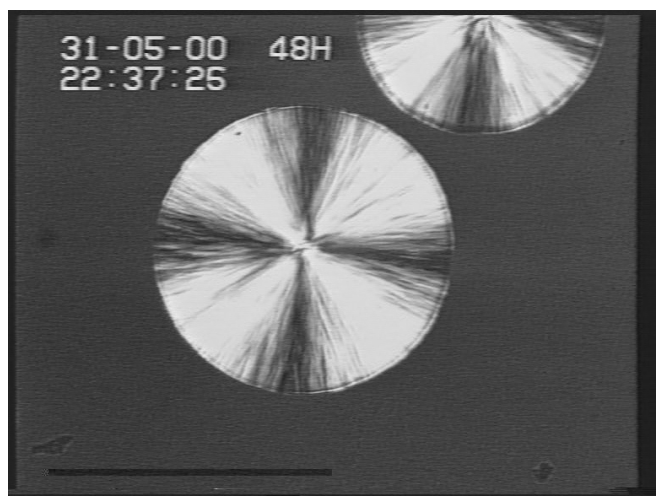

(a)

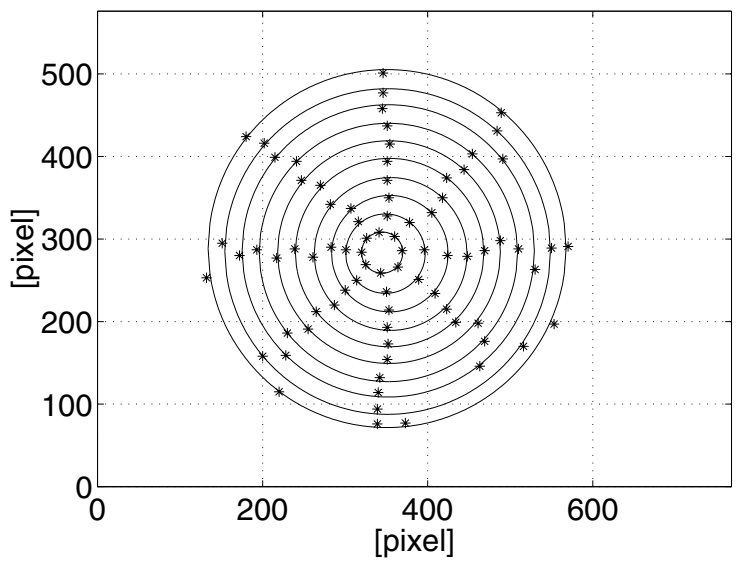

(b)

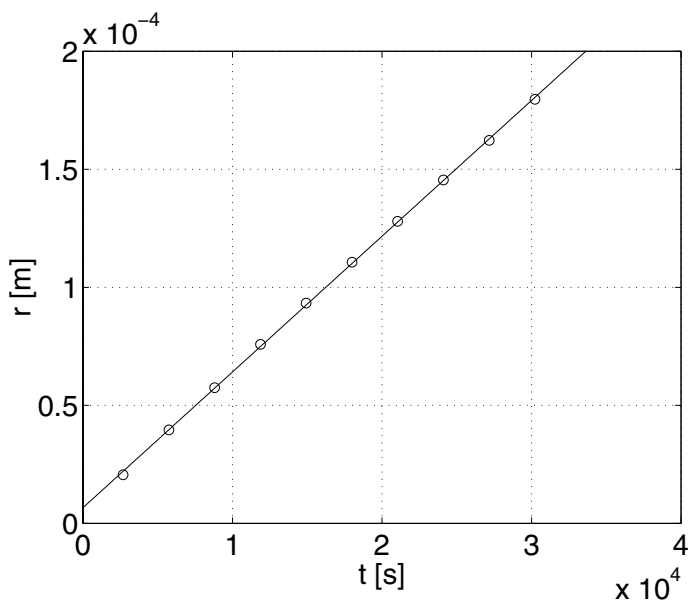

(c)

Figure 4.12: (a) DSM15M10 after $6^{\circ}: 41^{\prime}: 46^{\prime \prime}$ at $\mathrm{T}_{\mathrm{c}}=143.6\left[{ }^{\circ} \mathrm{C}\right]$. (b) Several circular fits corresponding to the edge of the spherulite. Pixel $(1,1)$ is positioned in the upper left corner of the image in (a) (c) Linear fit of the radii in time gives a growth rate of $5.75 \cdot 10^{-9}[\mathrm{~m} / \mathrm{s}]$.

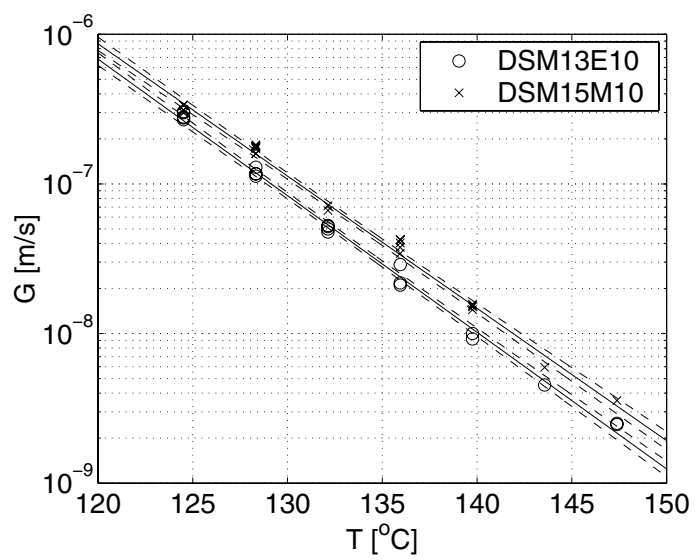

\begin{tabular}{|l|l|l|}
\hline & DSM13E10 & DSM15M10 \\
\hline \hline $\mathrm{c}_{\mathrm{G}}\left[\mathrm{K}^{-1}\right]$ & 0.210 & 0.204 \\
\hline $\mathrm{c}_{\mathrm{G} \min }\left[\mathrm{K}^{-1}\right]$ & 0.204 & 0.196 \\
\hline $\mathrm{c}_{\mathrm{G} \max }\left[\mathrm{K}^{-1}\right]$ & 0.217 & 0.211 \\
\hline \hline $\mathrm{G}_{\text {ref }}[\mathrm{m} / \mathrm{s}]$ & $1.019 \cdot 10^{-8}$ & $1.473 \cdot 10^{-8}$ \\
\hline $\mathrm{G}_{\text {ref } \min }[\mathrm{m} / \mathrm{s}]$ & $9.547 \cdot 10^{-9}$ & $1.367 \cdot 10^{-8}$ \\
\hline $\mathrm{G}_{\text {ref } \max }[\mathrm{m} / \mathrm{s}]$ & $1.089 \cdot 10^{-8}$ & $1.587 \cdot 10^{-8}$ \\
\hline
\end{tabular}

Figure 4.13: Fits and parameters of the fits for the growth curve of both DSM13E10 and DSM15M10 (reference temperature $\mathrm{T}_{\text {cref }}=140\left[{ }^{\circ} \mathrm{C}\right]$ ). The dashed lines are the $95 \%$ confidence interval for the two fits. Parameters with underscore ' $\mathrm{min}^{\prime}$ ' and ' $\mathrm{max}^{\prime}$ describe the $95 \%$ confidence interval for the two parameters at $\mathrm{T}_{\text {cref. }}$. 
temperature in the four outer points is $0.9\left[{ }^{\circ} \mathrm{C}\right]$. The temperature in all the nucleation measurements was shifted with this value.

\begin{tabular}{|l||r|r|r|}
\hline Point & $\mathrm{N}$ & $\mathrm{G}[\mathrm{m} / \mathrm{s}]$ & $\mathrm{T}\left[{ }^{\circ} \mathrm{C}\right]$ \\
\hline \hline 1 & 8 & $3.22 \cdot 10^{-9}$ & 147.6 \\
\hline 2 & 10 & $3.57 \cdot 10^{-9}$ & 147.1 \\
\hline 3 & 14 & $3.99 \cdot 10^{-9}$ & 146.5 \\
\hline 4 & 8 & $3.77 \cdot 10^{-9}$ & 146.8 \\
& & $3.66 \cdot 10^{-9}$ & 147.0 \\
\hline
\end{tabular}

Table 4.5: Measured number of nuclei and determined local temperature using a $4 \times$ objective at a hot-stage temperature of 147.9 [ $\left.{ }^{\circ} \mathrm{C}\right]$. Mean temperature in the four points is equal to $147.0\left[{ }^{\circ} \mathrm{C}\right]$.

The number of nuclei was assumed to be an unique function of temperature, and was described by

$$
N(T)=N_{\text {ref }} \exp \left\{-c_{N}\left(T-T_{\text {cref }}\right)\right\}
$$

with $\mathrm{N}_{\text {ref }}$ the number of nuclei at the crystallization reference temperature $\mathrm{T}_{\text {cref }}$ and $\mathrm{c}_{\mathrm{N}}$ a constant. These constants were estimated with a least squares fit of Equation (4.10). This equation is realistic for athermal nucleation, in which nuclei already exists at a high temperature, but reach their critical size at a certain supercooling (Eder and Janeschitz-Kriegl [35]). Notice that this equation gives a non-zero, but small value for the number of nuclei at temperatures above the melting temperature. Therefore, the growth rate is set equal to zero for temperatures above $163.4\left[{ }^{\circ} \mathrm{C}\right]$ in the calculations shown in this thesis.

As the distribution in spherulite size was small, it is concluded that nucleation occurred simultaneous after reaching the crystallization temperature. Moreover, it indicates that temperature gradients in each sample spot were small. The number of nuclei as a function of temperature in the four outer points, together with their fits, are shown in Figure 4.14. There are two differences in the nucleation curves. First, the slope for DSM13E10 is less steep. Second, the number of nuclei is higher for DSM13E10 for the same temperature.

\subsection{DSC}

\section{Crystallization}

DSC experiments were performed on a Perkin Elmer DSC Pyris 1. Isothermal crystallization was monitored between 126 and $140\left[{ }^{\circ} \mathrm{C}\right]$. A flexibele pan having a weight of about 26 [mg] was folded around a granulate piece of about 4.5 [mg]. Samples were annealed at $240\left[{ }^{\circ} \mathrm{C}\right]$ for 20 minutes and were cooled using a cooling rate of about $90\left[{ }^{\circ} \mathrm{C} / \mathrm{min}\right]$. The temperature calibration was checked before starting the measurements by determining the melting point of Indium $\left(156.6\left[{ }^{\circ} \mathrm{C}\right]\right)$ during a $10\left[{ }^{\circ} \mathrm{C} / \mathrm{min}\right]$ heating scan from 100 to $170\left[{ }^{\circ} \mathrm{C}\right]$. 


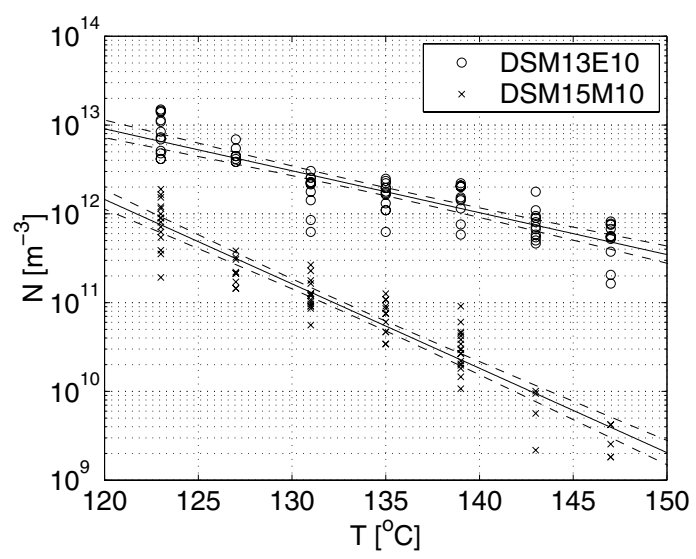

\begin{tabular}{|l|l|l|}
\hline & DSM13E10 & DSM15M10 \\
\hline \hline $\mathrm{c}_{\mathrm{N}}\left[\mathrm{K}^{-1}\right]$ & 0.109 & 0.219 \\
\hline $\mathrm{c}_{\mathrm{N} \min }\left[\mathrm{K}^{-1}\right]$ & 0.095 & 0.202 \\
\hline $\mathrm{c}_{\mathrm{N} \max }\left[\mathrm{K}^{-1}\right]$ & 0.122 & 0.236 \\
\hline \hline $\mathrm{N}_{\text {ref }}\left[\mathrm{m}^{-3}\right]$ & $1.031 \cdot 10^{12}$ & $1.825 \cdot 10^{10}$ \\
\hline $\mathrm{N}_{\text {ref min }}\left[\mathrm{m}^{-3}\right]$ & $9.102 \cdot 10^{11}$ & $1.538 \cdot 10^{10}$ \\
\hline $\mathrm{N}_{\text {ref } \max }\left[\mathrm{m}^{-3}\right]$ & $1.167 \cdot 10^{12}$ & $2.165 \cdot 10^{10}$ \\
\hline
\end{tabular}

Figure 4.14: Fits and parameters of the fits for the nucleation curve of both DSM13E10 and DSM15M10 (reference temperature $\mathrm{T}_{\text {cref }}=140\left[{ }^{\circ} \mathrm{C}\right]$ ). The dashed lines are the $95 \%$ confidence interval for the two fits. Parameters with underscore ' $m i n$ ' and ' $\mathrm{max}^{\prime}$ describe the $95 \%$ confidence interval for the two parameters at $\mathrm{T}_{\text {cref }}$.

Pogodina and Winter [115] used the Avrami equation for isothermal spherulitical crystallization with all nuclei available at $\mathrm{t}=0$ [s], to fit their DSC data:

$$
\frac{\chi}{\chi_{\infty}}=1-\exp \left\{-\left(\frac{t}{t_{A}}\right)^{3}\right\}
$$

where $\chi_{\infty}$ is the maximum crystallinity at long times and $t_{A}$ is the Avrami time, the time by which the crystallinity has reached $63 \%$ of the final crystallinity. They fitted the Avrami time as function of the supercooling with an exponential function

$$
t_{\text {A Pogodina }}=A \exp \left\{-B\left(T_{m}-T_{c}\right)\right\}
$$

Pogodina had chosen the melting temperature equal to $163\left[{ }^{\circ} \mathrm{C}\right]$. However, temperature $T_{m}$ is nothing else than a reference temperature to fit the data. It is much more convenient to choose a reference temperature close to the measurement temperatures. Therefore, the same reference temperature $T_{\text {cref }}$ is chosen as for the $N(T)$ and $G(T)$ fits:

$$
t_{A}=A \exp \left\{-B\left(T_{\text {cref }}-T_{c}\right)\right\}
$$

Using the assumption of Boutahar et al. $[18,19]$ that space filling and relative crystallinity are equal for polypropylene, the relation between the Avrami time and nucleation and growth is obtained by combining Equations (3.27) and (4.11) (see also Eder and Janeschitz-Kriegl [35, Section 5.1.3])

$$
t_{A}=\frac{1}{\left(\frac{4 \pi}{3} N\right)^{\frac{1}{3}} G}
$$

Using Equations (4.8) and (4.10), the coefficients A and B can be expressed in terms of the number of nuclei and the growth rate:

$$
\begin{aligned}
A & =\frac{1}{\left(\frac{4 \pi}{3} N_{r e f}\right)^{\frac{1}{3}} G_{r e f}} \\
B & =\frac{1}{3}\left(c_{N}+3 c_{G}\right)
\end{aligned}
$$


The Avrami time for both materials and their fits are shown in Figure 4.15 as a function of the crystallization temperature. Crystallization proceeds faster (shorter Avrami time) for DSM13E10. This can mainly be explained by the larger amount of nuclei in DSM13E10 (Figure 4.14). The parameters of the fits are of the same order of magnitude as the materials of Pogodina and Winter [115] $\left(A=2.66 \cdot 10^{6}[\mathrm{~s}]\right.$ and $\left.B=0.224[1 / \mathrm{K}]\right)$.

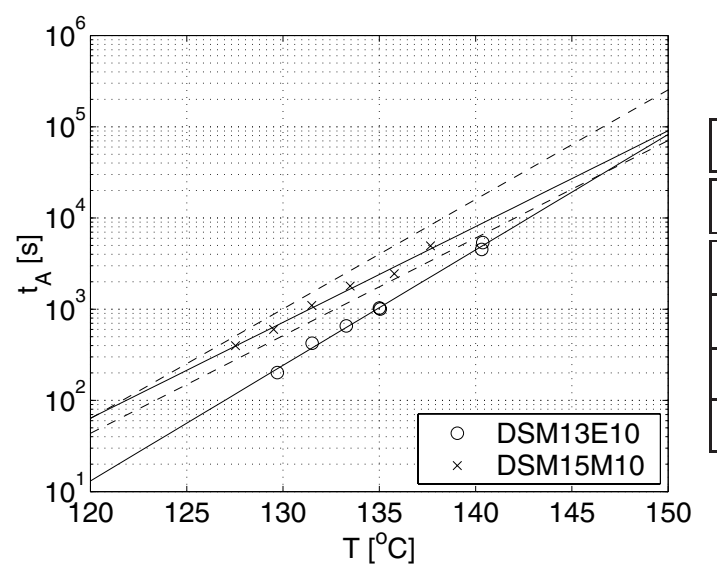

\begin{tabular}{|l|l|l|l|}
\hline & DSM13E10 & DSM15M10 & Remark \\
\hline \hline A [s] & $3.66 \cdot 10^{6}$ & $2.10 \cdot 10^{6}$ & Eq. 4.12 \\
\hline \hline A [s] & $4.47 \cdot 10^{3}$ & $8.06 \cdot 10^{3}$ & DSC fit \\
\hline A [s] & $6.02 \cdot 10^{3}$ & $1.60 \cdot 10^{4}$ & Eq. 4.15 \\
\hline B [1/K] & 0.292 & 0.242 & DSC fit \\
\hline B [1/K] & 0.246 & 0.277 & Eq. 4.16 \\
\hline
\end{tabular}

Figure 4.15: Fits and parameters of the fits for the Avrami time $t_{\mathrm{A}}$ determined from DSC data, for both DSM13E10 and DSM15M10. The dashed lines are the predictions using the nucleation and growth data (see text).

The coefficients A and B determined using the nucleation and growth data are also given in Figure 4.15. The Avrami time for the hot-stage data is larger than for the DSC experiments. The temperature dependence described by coefficient B is for both methods about the same. Coefficient A is higher for the hot-stage data, for both materials. The reference temperature of the hot-stage data should be shifted to $142.1\left[{ }^{\circ} \mathrm{C}\right]$ for DSM13E10 and 141.5 [ ${ }^{\circ} \mathrm{C}$ ] for DSM15M10 to obtain a comparable Avrami time for both methods (reference temperature was equal to $140\left[{ }^{\circ} \mathrm{C}\right]$ for both materials).

The absolute crystallinity of both materials was determined to be about $50 \%$ at the crystallization temperatures investigated, using a melt enthalpy of $207.1 \mathrm{~J} / \mathrm{g}$ ] for $100 \%$ crystalline $\alpha$-phase of iPP (Varga [141]). This amount is quite normal for iPP (see for example Gahleitner et al. [45]).

\section{Morphology}

The discrepancy in crystallization rate between the DSC and hot-stage data is investigated in more detail by looking at the morphology. The morphology developed in the DSC experiment at $140\left[{ }^{\circ} \mathrm{C}\right]$ was investigated by light microscopy for DSM13E10. A cross-section of the sample is shown in Figure 4.16(a-b). The mean radii determined from several spherulites in these cross-sections were equal to 59 and $69[\mu \mathrm{m}]$. The mean value for all spherulites in both cross-sections is equal to $63[\mu \mathrm{m}]$. No trans-crystalline layer was found in both cross-sections.

The Schneider rate equations are able to predict the structure of Figure 4.16(a-b) by using the experimental prescribed thermal history. However, the radius calculated by Schneider (Equation (3.23)) cannot be compared directly with the observed radius in microscopy, because Schneider gives the unbounded radius (i.e. the radius obtained 


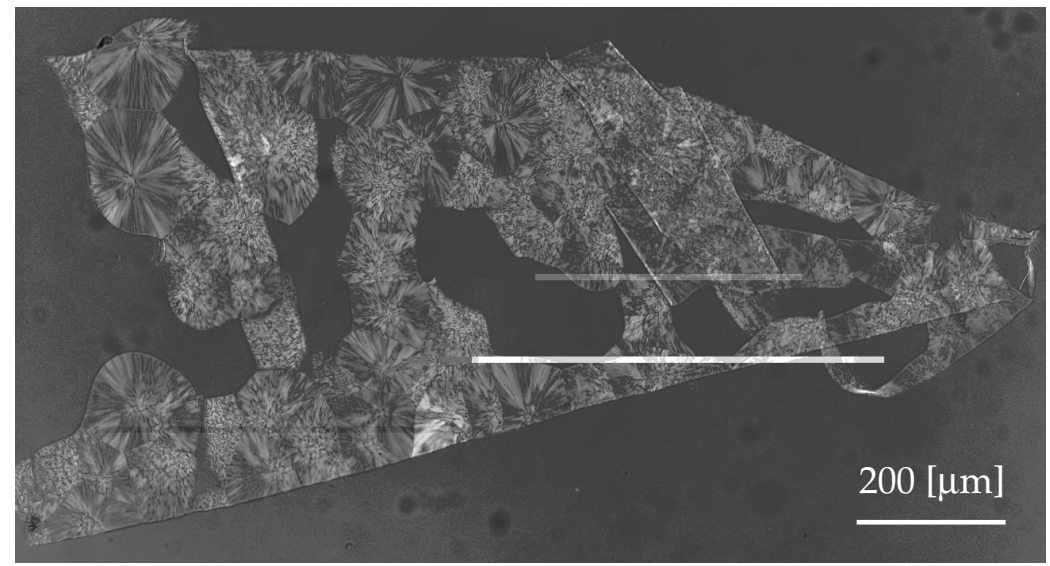

(a)

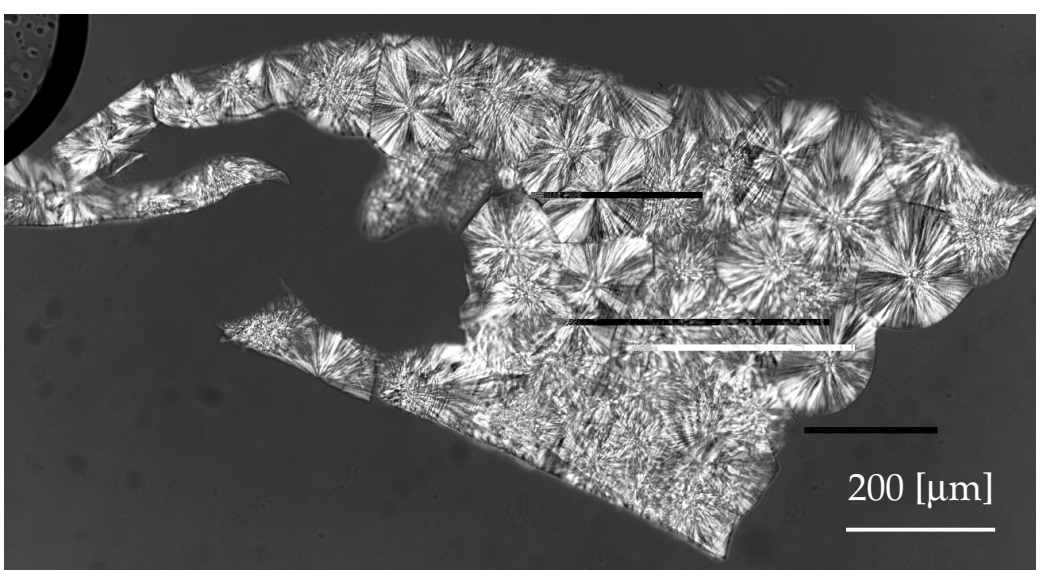

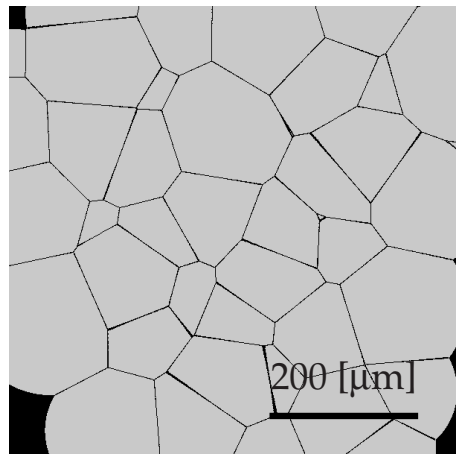

(c)

(b)

Figure 4.16: DSM13E10 crystallized in a DSC pan at $140\left[{ }^{\circ} \mathrm{C}\right]$. (a) Cross section of the sample. The average radius of six spherulites was about $59[\mu \mathrm{m}] .(\mathbf{b})$ Another cross-section of the same sample. The average radius of six spherulites was about $69[\mu \mathrm{m}]$. (c) Numerical simulation of the impinged structure. Average radius of the five largest spherulites is about $68[\mu \mathrm{m}]$.

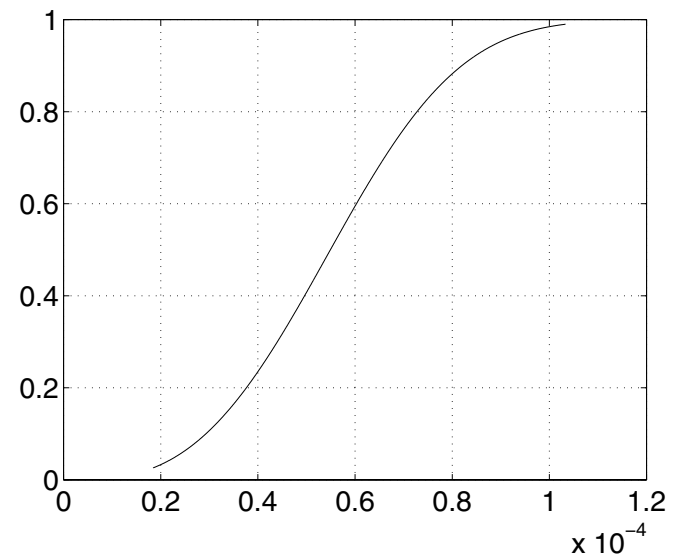


without impingement). Impingement was taken into account by simulating the nucleation and growth of spherulites in a three dimensional space. First, the Schneider equations were solved to obtain the sum of the radii $R_{\text {tot }}$ in time. Next, nuclei were inserted at random positions in a cube $\left(0.6[\mathrm{~mm}] \times 0.6[\mathrm{~mm}] \times 2 \mathrm{R}_{\text {tot }} / \mathrm{N}\right)$, and growth was simulated (according to $\mathrm{R}_{\text {tot }}(\mathrm{t})$ ). Intersection of the spherulites with the center plane of the cube was determined. This plane was divided in $600 \times 600$ pixels. Impingement of spherulites was defined if two spherulites occupy the same pixel in the same time step with the restriction that this pixel was not occupied by a spherulite. The increase of the radius per time step should not be larger than the pixel size to obtain a noninterrupted line. The resulting structure after almost complete space filling is given in Figure 4.16(c). The mean radius determined from the five largest spherulites, which are not at the edge of the image, was about $68[\mu \mathrm{m}]$. A quantitative, good agreement of the structure is obtained between the simulation and the cross-section of Figure 4.16(b). The structure of Figure 4.16(a) has been subjected to a lower temperature, resulting in smaller spherulites.

Visualisation of the structure as described in the previous paragraph, takes much more computing time than solving the Schneider rate equations only. However, the maximum packing fraction obtained in the numerical simulation can be used to determine the bounded radius for other thermal histories. The space filling corresponding to a maximum, bounded radius of the simulation $(68[\mu \mathrm{m}])$ is equal to 0.73 [-] (see Figure 4.17). This value is reasonably close to the maximum packing fraction of 0.64 for a random closed packing known from dispersion rheology (Barnes et al. [8]). The difference is probably caused by the determination of the maximum (bounded) radius. The experimental and numerical radii in Figure 4.16 were determined by fitting circles on the spherulite boundaries. (same procedure as was used in Figure 4.12).

Using this maximum packing fraction of $0.73[-]$, the difference in temperature can be calculated to end up with the mean radius obtained from the two cross-sections in Figure 4.16(a-b) $(63[\mu \mathrm{m}])$. Variation of the reference temperature showed that a radius of $63[\mu \mathrm{m}]$ and a space filling of 0.73 corresponds to a reference temperature of $142.1\left[{ }^{\circ} \mathrm{C}\right]$. This agrees with the earlier observation that $\mathrm{T}_{\text {cref }}$ has to be shifted to higher temperatures to obtain the same Avrami time for both DSC and the hot-stage data.

Despite the large efforts to correct the measured data using an 'accurate' temperature calibration, still a deviation in crystallization rate was found between the DSC and hot-stage data. Agreement between these two sets of data was obtained by shifting the reference temperature of the hot-stage data to about $141.8\left[{ }^{\circ} \mathrm{C}\right]$. This was checked by microscopy and numerical calculation of the structure.

\subsection{Rheometry}

Isothermal crystallization during quiescent conditions and after short time shearing was monitored on a rotational viscometer (ARES 3LS-4A, Rheometrics Inc.) using oscillatory experiments. A cone-plate geometry having a diameter of $25[\mathrm{~mm}]$ and a cone angle of 0.1004 [rad] was used. Smaller samples having a diameter of 10.5 [mm] were used for shear rates larger than $5\left[\mathrm{~s}^{-1}\right]$. A new sample was used for each experiment. The quiescent experiments consisted of the following steps: 
1. annealing the sample in a nitrogen atmosphere.

2. cooling the sample to the crystallization temperature $T_{c}$ using liquid nitrogen.

3. monitoring crystallization using an air environment between 134 and $144\left[{ }^{\circ} \mathrm{C}\right]$ for a single frequency $\omega=10[\mathrm{rad} / \mathrm{s}]$.

The shear-induced experiments consisted of the following steps:

1. annealing the sample in a nitrogen atmosphere.

2. cooling the sample to the flow temperature $\mathrm{T}_{\mathrm{f}}$ using liquid nitrogen.

3. shearing the sample for a short time $\left(\dot{\gamma} \leq 50\left[\mathrm{~s}^{-1}\right]\right.$ and $\left.\mathrm{t} \leq 40[\mathrm{~s}]\right)$.

4. if $\mathrm{T}_{\mathrm{f}}$ not equal to $\mathrm{T}_{\mathrm{c}}$ : cool sample to $\mathrm{T}_{\mathrm{c}}$ using liquid nitrogen.

5. monitoring crystallization using an air environment at $140\left[{ }^{\circ} \mathrm{C}\right]$ for a single frequency $\omega=10[\mathrm{rad} / \mathrm{s}]$.

Samples were annealed at $240\left[{ }^{\circ} \mathrm{C}\right]$ for 20 minutes in a nitrogen environment. The maximum cooling rate was about $65\left[{ }^{\circ} \mathrm{C} / \mathrm{min}\right]$. An undershoot of about 1.5-2.5 [ $\left.{ }^{\circ} \mathrm{C}\right]$ was observed in all experiments on cooling to the desired crystallization temperature $\left(\mathrm{T}_{\mathrm{c}}\right)$. The temperature fluctuation during isothermal crystallization was within $0.1\left[{ }^{\circ} \mathrm{C}\right]$. The moment the sample was set to cool from $240\left[{ }^{\circ} \mathrm{C}\right]$ to the desired crystallization temperature, was defined as time $t=0$ [s]. Shearing was started after 200 [s] and 1000 [s]. With the latter, the influence of nucleation and crystallization on the rheological behavior can be investigated. Relaxation of the stresses was monitored for 200 [s] after the shear step. The flow temperature was varied between 140 and $200\left[{ }^{\circ} \mathrm{C}\right]$ for DSM15M10 only.

The zero gap height of the tool at $T_{c}$ and, in case of flow, the position of the motor of the rheometer, were determined before inserting a sample. The gap height was adjusted manually in order to correct for the thermal expansion of the tool $\left(2.4\left[\mu \mathrm{m} /{ }^{\circ} \mathrm{C}\right]\right)$. The dynamic part of the experiment can only be performed in the zero position of the motor. A gentile transition from the static into the dynamic mode of the motor could be obtained only if the motor is exactly in the zero position at the end of the stationary part of the experiment. Therefore, the motor was moved from the zero position using the same shear rate and strain as in the experiment to follow, but in the opposite direction, before inserting a sample.

The temperature of the rheometer was calibrated with a small piece of Indium (about 22 [mg]; $\mathrm{T}_{\mathrm{m}}=156.6\left[{ }^{\circ} \mathrm{C}\right]$ ). The Indium was cooled with $0.1\left[{ }^{\circ} \mathrm{C} / \mathrm{min}\right]$ starting from $165\left[{ }^{\circ} \mathrm{C}\right]$, while performing a dynamic temperature ramp test with a strain of $2 \%$ and a frequency of $10[\mathrm{rad} / \mathrm{s}]$. The melting temperature was detected as a sharp increase in the torque (at $154.9\left[{ }^{\circ} \mathrm{C}\right]$ ).

Gottlieb and Macosko [55] and Macosko [88] discussed the effect of instrument compliance on dynamic rheological measurements. Compressive compliance is negligible, but torsional compliance can be important when the sample torsional stiffness is high relative to the instrument stiffness. The torsional compliance of the force rebalance transducer of the ARES was equal to $1.33 \cdot 10^{-6}[\mathrm{Nm} / \mathrm{rad}]$. If stiffness of the 
sample approaches the spring constant of the instrument (1/compliance) the transducer became instable. Therefore, small sample sizes should be used to measure the final crystallization plateau as observed by Boutahar et al. [19] or Vleeshouwers and Meijer [147]. The transducer showed instabilities above dynamic moduli of $1 \cdot 10^{6}$ and $2 \cdot 10^{7}[\mathrm{~Pa}]$ for the 25 and $10.5[\mathrm{~mm}]$ diameter samples, respectively.

In the next part of this section, relaxation after flow, crystallization, as monitored with oscillatory experiments, and morphology are discussed in more detail.

\section{Relaxation}

Doufas et al. [29] proposed a model in which the relaxation time of the amorphous phase decreases with the progress of crystallization due to the loss of chain segments, while Zuidema et al. [157] proposed a model in which it increases due to network formation. It is interesting to investigate whether such rheological changes can be seen in the start-up and cessation part of the experiments .

The transient viscosity and the first normal stress difference were monitored after a shear-rate step at $140\left[{ }^{\circ} \mathrm{C}\right]$, which was applied 200 and 1000 [s] after starting the cooling. In the second case, nuclei have already grown out into small spherulites. The transient viscosity for DSM13E10 after 200 [s], and applying several shear rates for 1 [s], is shown in Figure 4.18(a). In this figure, also the predicted curves are given for the Leonov and Pompom model without any coupling with crystallization. The relaxation behavior is described reasonably well by both Leonov and Pompom for the low shear rates (up to $10\left[\mathrm{~s}^{-1}\right]$ ), while it is different for the two highest shear rates (20 and $\left.50\left[\mathrm{~s}^{-1}\right]\right)$. The relaxation time has been increased, probably due to the formation of flow-induced nuclei. Moreover, a small upswing in the viscosity is seen for the highest shear rate.

The transient viscosity for DSM13E10 after 1000 [s], and applying a shear rate of $2.5\left[\mathrm{~s}^{-1}\right]$ for several shear times are shown in Figure 4.18(b). It seems that the rheological behavior has not been changed due to the larger waiting time. The Pompom model under-predicts and the Leonov model over-predicts the relaxation slightly. It is important to notice that edge failure is not observed in the experiments shown (no decrease in viscosity during shearing).

The startup behavior of the first normal stress difference $\mathrm{N}_{1}$ during short time shearing (50 [s ${ }^{-1}$ ] for 1 [s]) of DSM15M10 at 160,180 and $200\left[{ }^{\circ} \mathrm{C}\right]$ is shown in Figure 4.19. A small increase in $\mathrm{N}_{1}$ is observed after the usually observed overshoot. This small increase was not observed for shear rates lower than 20 [s $\left.{ }^{-1}\right]$. An increase of $\mathrm{N}_{1}$ during shearing was also observed by Kumaraswamy et al. [76] in rheo-optical measurements. He attributed this increase to the creation of a 'shear-induced structure' (as discussed in Section 2.3). The increase in $\mathrm{N}_{1}$ observed in the rheometer might be attributed to the dynamic behavior of the transducer, because the experimental conditions, a large shear rate for only one second, are quite extreme.

In conclusion, small changes in rheology are found in the first normal stress difference during shear, and the relaxation of the viscosity after shear, only for the higher shear rates. 


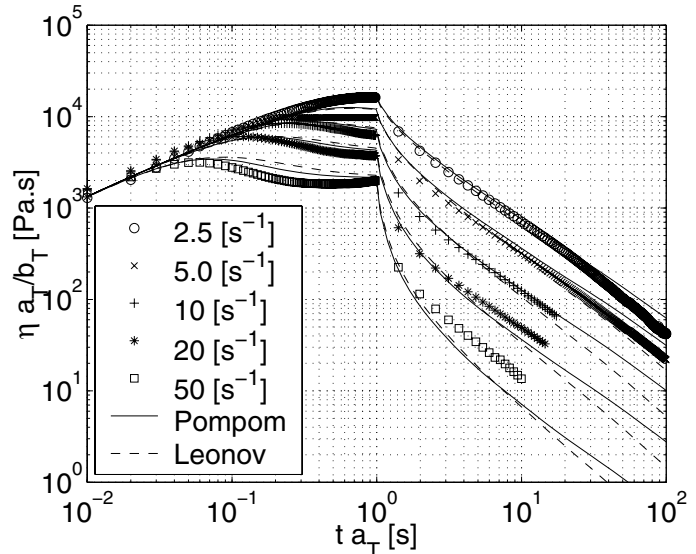

(a)

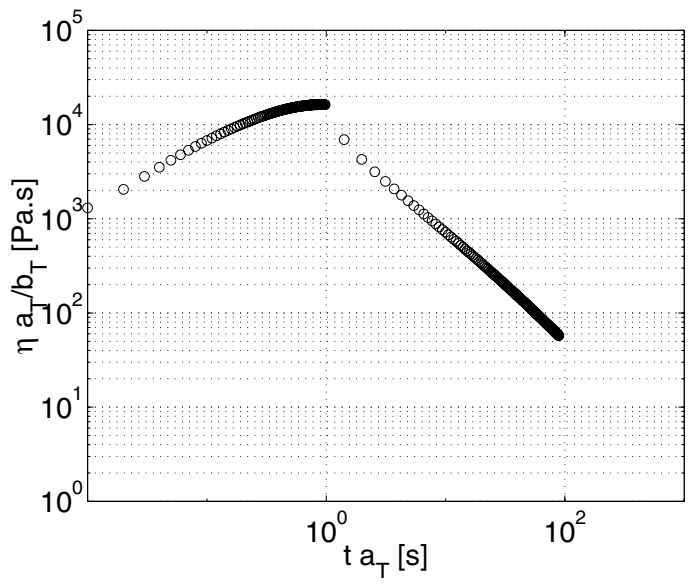




\section{Crystallization}

An example of the development of the storage modulus during quiescent crystallization is shown in Figure 4.20(a). The modulus increases during cooling from 240 to $140\left[{ }^{\circ} \mathrm{C}\right]$. Next, it shows an overshoot during the undershoot in temperature and increases after 1500 [s] due to the developing structure. It is important to choose a low oscillatory strain, because shear should not interfere with and enhance crystallization. Moreover, the auto-strain option was used to limit the maximum torque applied to the sample. The strain was decreased $10 \%$ if the torque became larger than $1 \cdot 10^{-3}[\mathrm{Nm}]$. A strain of $0.4 \%$ was chosen and its influence was checked by another experiment, in which the sample was applied to the same thermal history and in which the monitoring of crystallization was started after 3000 [s]. Figure 4.20(a) shows that the applied strain does not influence crystallization. The influence of the sample size is also shown in Figure 4.20(a). The sample with a diameter of 10.5 [mm] shows the same behavior, but was influenced less by the instrument compliance, resulting in a longer measurement time. The deviation close to a modulus of $1 \cdot 10^{6}[\mathrm{~Pa}$ can be explained by the instrument compliance for the large samples.

Direct comparison of the relation proposed by Khanna (Equation (2.2)) and the prediction for the Schneider rate equations, is shown in Figure 4.20(b). The maximum modulus $\mathrm{G}_{\infty}^{\prime}$ in Figure 4.20(a) was estimated to be about $5 \cdot 10^{7}[\mathrm{~Pa}$ ]. The predictions differ in the magnitude for the space filling and the shape of the curves. According to the relation of Khanna, space filling increases largly when the modulus approaches the final modulus. This highly non-linear relation seems, in comparison with Schneider, not to be a good description for the space filling.

Pogodina and Winter [115] showed an earlier increase in the modulus at low frequencies $\left(10^{-2}[\mathrm{rad} / \mathrm{s}]\right)$ than at higher frequencies (up to $1[\mathrm{rad} / \mathrm{s}]$ ). Important for these measurements is that the rate of crystallization is slow enough to ensure that the modulus does not change much during the determination of a single measurement point. Therefore, the time-consuming, low frequency measurements are not useful at lower temperatures, especially when shearing is applied. A MultiWave experiment, in which moduli for several frequencies can be determined simultaneously, was performed to show the effect of frequency (Figure 4.21). It seems that the increase in $\mathrm{G}^{\prime}$ occurs at the same time for all frequencies. Therefore, a frequency of $10[\mathrm{rad} / \mathrm{s}]$ was chosen in all experiments.

Quiescent crystallization for both materials is shown in Figure 4.22. The faster crystallization behavior of DSM13E10 in comparison to DSM15M10 is caused by the larger number of nuclei (Figure 4.14). Vleeshouwers and Meijer [147] performed measurements on the same material, but observed faster crystallization for DSM15M10 (G' (10 $\left.[\mathrm{rad} / \mathrm{s}], 138\left[{ }^{\circ} \mathrm{C}\right]\right)=1 \cdot 10^{6}[\mathrm{~Pa}]$ at $\left.1630[\mathrm{~s}]\right)$ than DSM13E10 $\left(\mathrm{G}^{\prime}\left(10[\mathrm{rad} / \mathrm{s}], 138\left[{ }^{\circ} \mathrm{C}\right]\right)=\right.$ $1 \cdot 10^{6} \mathrm{~Pa}$ at $\left.3500[\mathrm{~s}]\right)$. This shows that the number of nuclei is dependent on the batch and that comparison of data from 'the same material' is not straight forward in crystallization studies. The difference in the time scale between data of Vleeshouwers and Figure 4.22 could be explained by a difference in the temperature. Further on, it seems that the maximum modulus $G_{\infty}^{\prime}$ observed by Vleeshouwers is lower.

The enhancement of crystallization with larger supercooling can be investigated by comparing the $\mathrm{G}^{\prime}$ data and the predictions by Schneider. This comparison is performed 

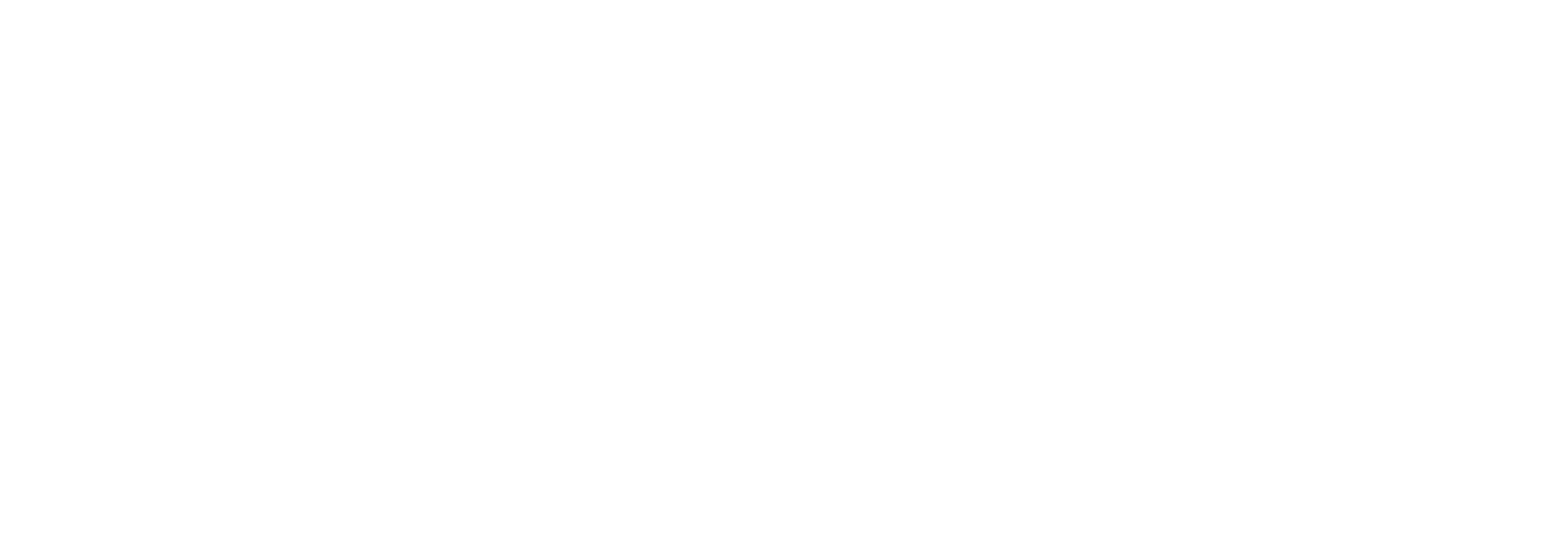

(a) 


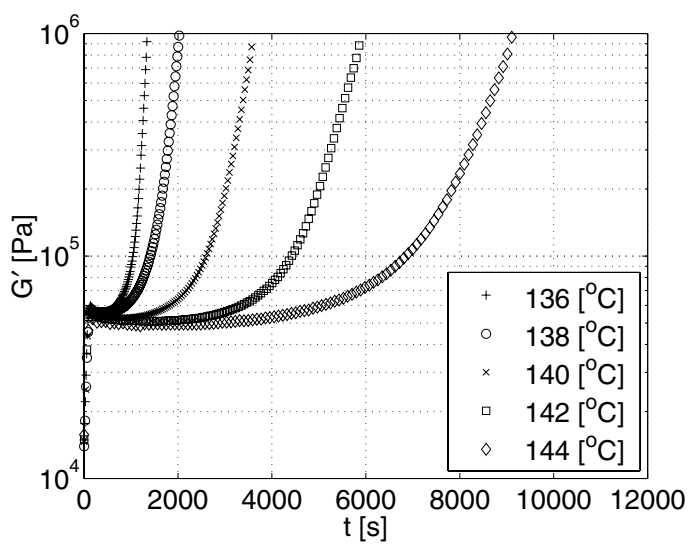

(a)

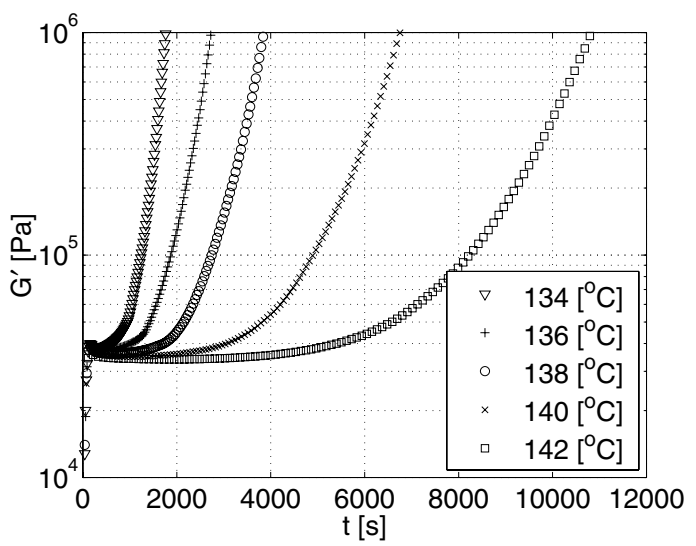

(b)

Figure 4.22: Quiescent rheological experiments for (a) DSM13E10 and (b) DSM15M10 $\left(\omega=10\left[\mathrm{~s}^{-1}\right]\right.$ and $\left.\gamma \leq 0.4[-]\right)$.

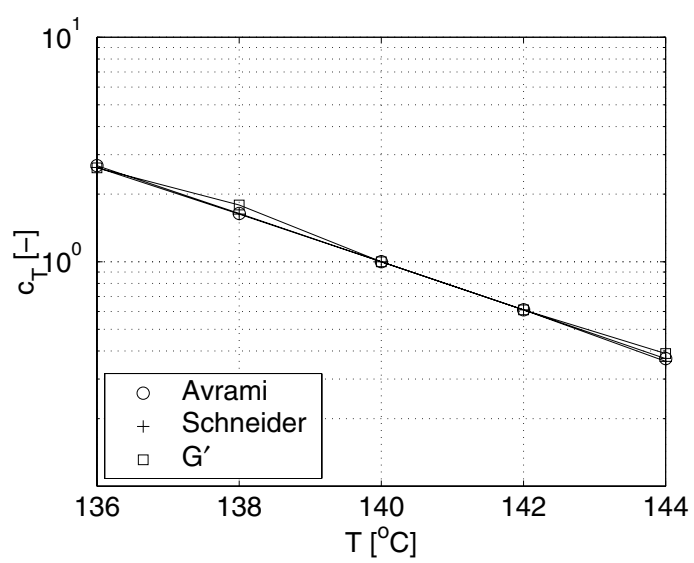

(a)

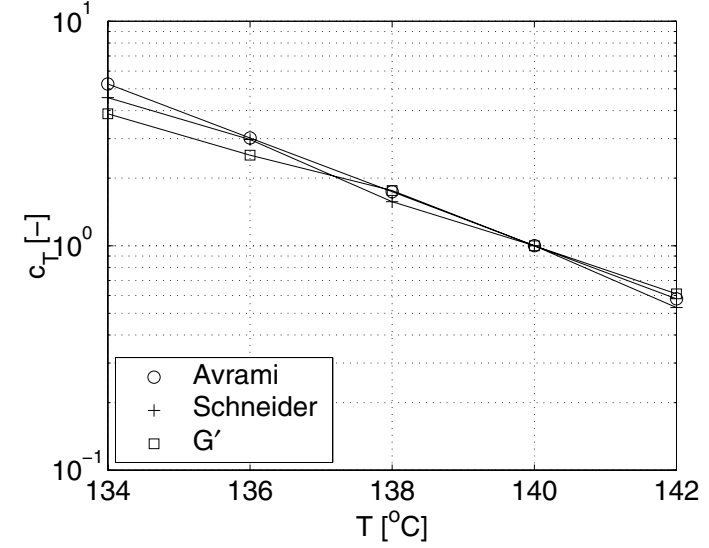

(b)

Figure 4.23: Crystallization shift factor $\mathrm{c}_{\mathrm{T}}$ predicted by Avrami (Equation (4.18)) and determined by shifting the Schneider predictions (in which the experimental thermal history was used) and the storage moduli (G'). (a) DSM13E10 and (b) DSM15M10.

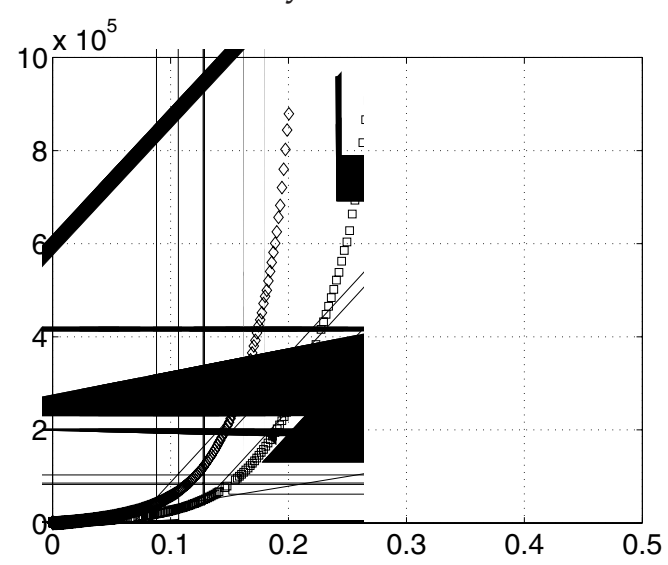


for a 'crystallization shift factor' $\mathrm{c}_{\mathrm{T}}$, which shifts the curves at different crystallization temperatures in time to one curve at the crystallization reference temperature $\mathrm{T}_{\text {cref, }}$, on a logaritmic scale. The storage moduli in Figure 4.22 are shifted horizontally to the measurement at $140\left[{ }^{\circ} \mathrm{C}\right]$ for both materials. The prediction of Schneider was calculated by using the experimental thermal histories of these experiments and were also shifted horizontally to the prediction at same reference temperature.

Some insight on the behavior of $\mathrm{c}_{\mathrm{T}}$ can be obtained by looking at the Avrami equation (Equation (3.27)), in which the expressions for nucleation and growth (Equation (4.8) and (4.10)) are inserted

$$
\xi_{g}=1-\exp \left[-\frac{4 \pi}{3} N_{\text {ref }} G_{r e f}^{3} t^{3} \exp \left\{-\left(c_{N}+3 c_{G}\right)\left(T-T_{\text {cref }}\right)\right\}\right]
$$

So, factor $\mathrm{c}_{\mathrm{T}}$ is given by

$$
c_{T}=\exp \left[-\frac{1}{3}\left(c_{N}+3 c_{G}\right)\left(T-T_{\text {cref }}\right)\right]
$$

and depends on the temperature dependence of nucleation and growth.

The shift factors given by Avrami (Equation (4.18)), Schneider and the $G^{\prime}$ data are given in Table 4.6 and shown in Figure 4.23. An excellent agreement between the three sets of shift factors is obtained for DSM13E10, while for DSM15M10 larger differences occur, especially at the lower temperatures. It should be noted that these measurements were performed at different temperatures with the same frequency. However, correction of the frequency will give only a small change in factor $\mathrm{c}_{\mathrm{T}}$ and does not explain the large differences observed for DSM15M10.

\begin{tabular}{|l|c|c||c|c|c|}
\hline \multirow{2}{*}{ Material } & \multirow{2}{*}{$\mathrm{T}_{\mathrm{c}}\left[{ }^{\circ} \mathrm{C}\right]$} & \multirow{2}{*}{$\Delta \mathrm{T}\left[{ }^{\circ} \mathrm{C}\right]$} & \multicolumn{3}{|c|}{$\mathrm{C}_{\mathrm{T}}[-]$} \\
\cline { 4 - 6 } & & & Avrami & Schneider & $\mathrm{G}^{\prime}$ \\
\hline \hline \multirow{4}{*}{ DSM13E10 } & 136 & 2.5 & 2.68 & 2.63 & 2.62 \\
\cline { 2 - 6 } & 138 & 2.7 & 1.64 & 1.63 & 1.79 \\
\cline { 2 - 6 } & 140 & 2.5 & 1.00 & 1.00 & 1.00 \\
\cline { 2 - 6 } & 142 & 2.5 & 0.61 & 0.61 & 0.61 \\
\cline { 2 - 6 } & 144 & 2.3 & 0.37 & 0.36 & 0.39 \\
\hline \hline \multirow{5}{*}{ DSM15M10 } & 134 & 1.5 & 5.26 & 4.57 & 3.87 \\
\cline { 2 - 6 } & 136 & 2.6 & 3.02 & 2.97 & 2.53 \\
\cline { 2 - 6 } & 138 & 1.5 & 1.74 & 1.57 & 1.76 \\
\cline { 2 - 6 } & 140 & 2.6 & 1.00 & 1.00 & 1.00 \\
\cline { 2 - 6 } & 142 & 1.3 & 0.58 & 0.53 & 0.61 \\
\hline
\end{tabular}

Table 4.6: Crystallization shift factor $c_{T}$ for both materials predicted by Avrami (Equation (4.18)) and determined by shifting the Schneider predictions (in which the experimental thermal history was used) and the storage moduli $\mathrm{G}^{\prime}$. Here, $\Delta \mathrm{T}$ is defined as the undershoot that was observed on cooling to the desired crystallization temperature $T_{c}$. 
The Avrami prediction should agree with Schneider, because the cooling rate is high (about $65\left[{ }^{\circ} \mathrm{C} / \mathrm{min}\right]$ ). A closer look at the undershoot in temperature of these experiments (Table 4.6) shows that larger differences in undershoot occur for DSM15M10. Since Avrami can not distinct between differences in undershoot, a deviation in the shift factor for Schneider and Avrami is found. The difference between the modulus and Schneider can be explained by an error in the temperature dependence of the nucleation or growth rate measurements. However, the value of about 0.2 for the temperature dependence of both the nucleation $\left(c_{N}\right.$, Equation (4.10)) and the growth rate $\left(c_{G}\right.$, Equation (4.8)) is quite normal for isotactic polypropylene.

The relation between $\mathrm{G}^{\prime}$ and space filling during crystallization is shown in Figure 4.24. Unfortunately, the measurements at different temperatures do not follow the same curve. This comparison is quite sensitive to experimental errors and a deviation occurs for the higher crystallization temperatures (an exception is the experiment on DSM13E10 at $\left.138\left[{ }^{\circ} \mathrm{C}\right]\right)$.

The enhancement of crystallization with increasing shear rates and strains was also determined by monitoring $G^{\prime}(t)$. Short time shearing at $140\left[{ }^{\circ} \mathrm{C}\right]$ for 1 [s] and several shear rates is shown in Figure 4.25. Again, a 'crystallization shift factor' $\mathrm{c}_{\mathrm{T}}$ was determined by shifting the flow-induced curves to the reference curve, which is the quiescent crystallization curve at $140\left[{ }^{\circ} \mathrm{C}\right]$. The factors for several experiments on both materials are shown in Figure 4.26. It shows that this factor is more sensitive to an increase in the shear rate than an increase in the strain. Moreover, DSM13E10 is more sensitive than DSM15M10 for shear rates less or equal to $5\left[\mathrm{~s}^{-1}\right]$. For a large shear rate of $20\left[\mathrm{~s}^{-1}\right]$, crystallization is more enhanced for DSM15M10 than for DSM13E10.

\section{Morphology}

The morphology in case of quiescent crystallization in DSM13E10 at $140\left[{ }^{\circ} \mathrm{C}\right]$ (see Figure 4.20) was investigated by light microscopy (Figure 4.27(a-d)). In all cross-sections, spherulites were found that were not impinged at the moment of quitting the experiment and opening the oven (at 5022 [s]). The maximum radius of these spherulites is about $52[\mu \mathrm{m}]$. Since the spread of these radii is quite small, it can be concluded that the crystallization temperature is quite homogeneous throughout the sample. Moreover, this size agrees excellent with the Schneider predictions from the $\mathrm{T}_{\text {cref }}$ corrected (see Section 4.4) hot-stage data. The cross-section close to the outside of the sample in Figure 4.27 (d) contains many spherulites that were not impinged. This can be seen as a change in the birefringence caused by a step in the growth. It seems that in this part less nuclei were created. This phenomenon is not understood.

Trans-crystallization occurs at the edges of the sample (for example Figure 4.27(c)). The thickness of this layer is about equal to the spherulite radius. Depending on the crystallization temperature, this layer can have a large effect on the modulus of the sample, because the gap of the cone-plate geometry is equal to $61[\mu \mathrm{m}]$.

The morphology in case of crystallization after short time shearing, using $2.5\left[\mathrm{~s}^{-1}\right.$ ] for 10 [s] in DSM13E10 at $140\left[{ }^{\circ} \mathrm{C}\right]$, was also investigated. A cross-section perpendicular and parallel to the shearing direction are shown in Figure 4.28(a) and (b), respectively. Three important features are present in these figures. First, the birefringence in Figure 4.28(a) is less pronounced than in the quiescent case. This could be due to 


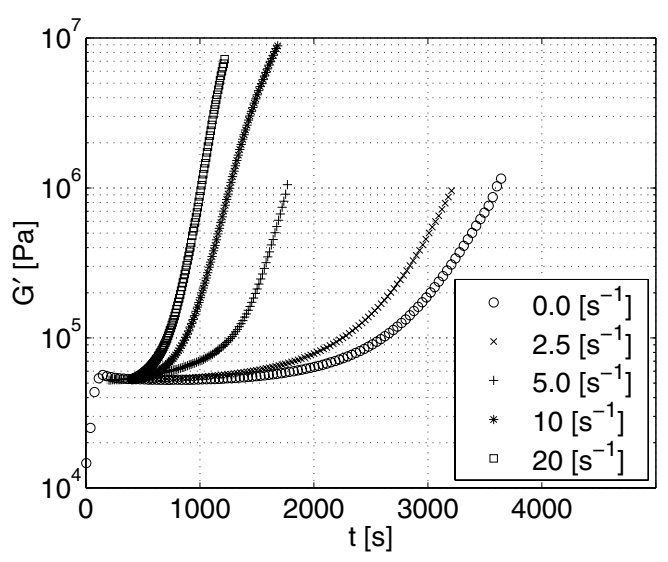

(a)

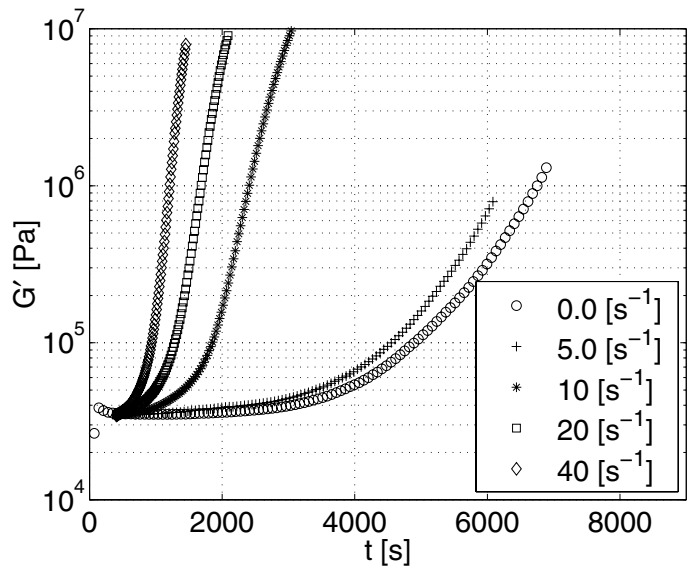

(b)

Figure 4.25: Development of the storage modulus after 1 [s] shearing at $140\left[{ }^{\circ} \mathrm{C}\right]$ for (a) DSM13E10 and (b) DSM15M10.

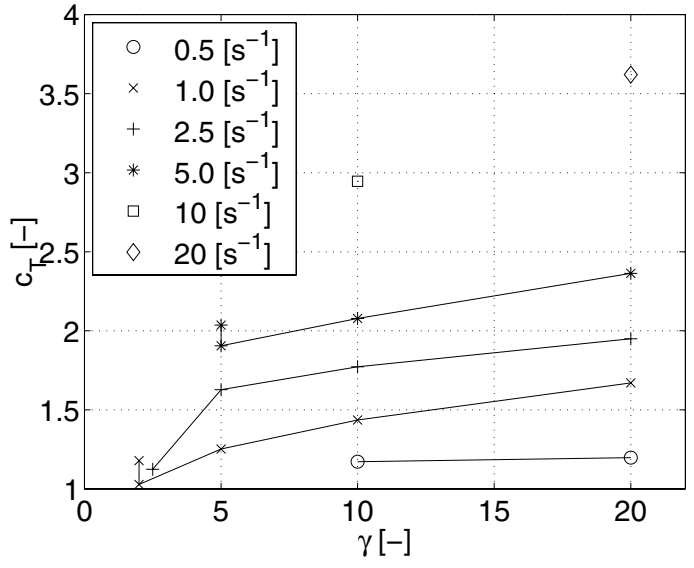

(a)

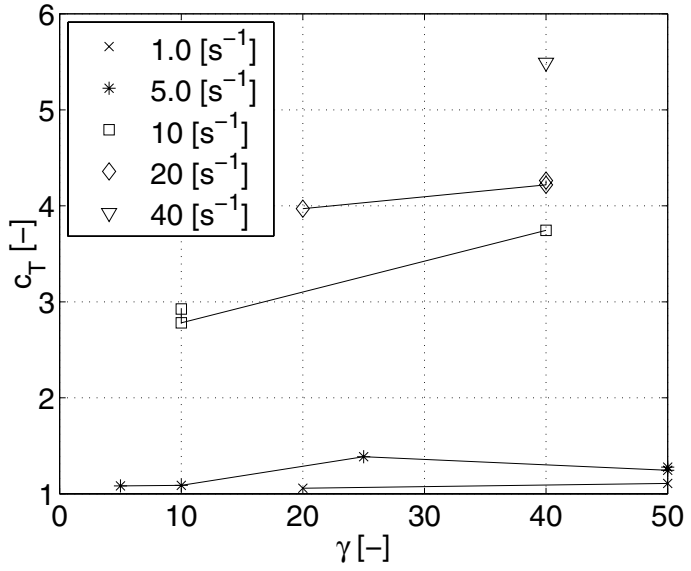

(b)

Figure 4.26: Crystallization shift factor $\mathrm{c}_{\mathrm{T}}$ for both materials after short time shearing at $140\left[{ }^{\circ} \mathrm{C}\right]$. Points having the same shear rate are connected with a line. (a) DSM13E10 and (b) DSM15M10. 
more lamellar branches, like observed at lower crystallization temperatures in quiescent conditions, or just a different thickness of the prepared light microscopy sample. The samples were cut with a glass knife below $\mathrm{T}_{g}$, and have a thickness of about $7[\mu \mathrm{m}]$ with an accuracy of about $1-2[\mu \mathrm{m}]$. It seems that the first explanation is true, but more validation is needed. Second, flow enhances nucleation as can be seen in the spherulitical size, which is about twice as small as in the quiescent case, but no oriented structures are seen in flow direction. Third, a row nucleated structure is observed perpendicular to the flow direction. This agrees with the observations of Jerschow and Janeschitz-Kriegl [66], who found oriented structures also in this direction. For higher deformations, this structure would orient in flow direction.

\subsection{Crystallization behavior}

\subsubsection{Fitting of the SOAS experiments}

The parameters of the $S_{\mathrm{J} 2}$ model for DSM13E10 are determined for the small oscillatory shear experiments (Figure 4.26(a)). Two assumptions were made for these experiments. First, the relaxation time $\tau_{\mathrm{n}}$ was chosen infinite, because the experimental temperature $\left(140\left[{ }^{\circ} \mathrm{C}\right]\right)$ is below the melting temperature. Therefore, the number of flow-induced nuclei is expected not to decrease. Second, the flow-induced nuclei will grow into spherulites. This will be true for the low shear rates applied for a short time. Row nucleation was shown for the experiment with a shear rate of $5\left[\mathrm{~s}^{-1}\right]$ applied for 1 [s]. However, the second assumption is used as a simplification in order to reduce the number of fitting parameters, and the model should thus be able to describe at least the lower curves in Figure 4.26.

Using these assumptions, only two parameters have to be fitted: the scaling factor for the second invariant $g_{n}^{\prime}$ and the coupling of the number of nuclei with the rheological relaxation time $\beta$. The number of flow-induced nuclei was added to the number of nuclei obtained in quiescent conditions. The deformation history was applied in the same way as in the experiments, while only one thermal history was used for all predictions calculated.

A first order implicit Euler scheme is used to determine the quiescent part $\left(\phi_{\mathrm{i}}\right)$ and the flow-induced part $\left(\psi_{\mathrm{i}}\right)$ of the crystallization. A logarithmic increasing time step was chosen for both the flow and relaxation part of the calculation, while the time steps for the crystallization part was chosen constant. The calculation was stopped at a space filling value of $0.95[-]$.

The influence of $\beta$ is shown in Figure 4.29. A value smaller than $\mathcal{O}\left(10^{-7}\right)$ will not influence the maximum rheological relaxation time, while larger values increase the rheological relaxation time and, therefore, the second invariant and the number of flow-induced nuclei (Figure 4.29(a)). This self-enhancing effect can be too strong for a large $\beta$ due to the unbounded maximum relaxation time. The influence of $\beta$ on the crystallization rate is shown in Figure 4.29(b). Parameter $\beta$ increases the sensitivity of the crystallization rate on total shear. The influence of $g_{n}^{\prime}$ is shown in Figure 4.30. The slope increases considerably for larger values of $g_{n}^{\prime}$.

The small oscillatory shear experiments cannot be predicted well by the $S_{\mathrm{J} 2}$ model 


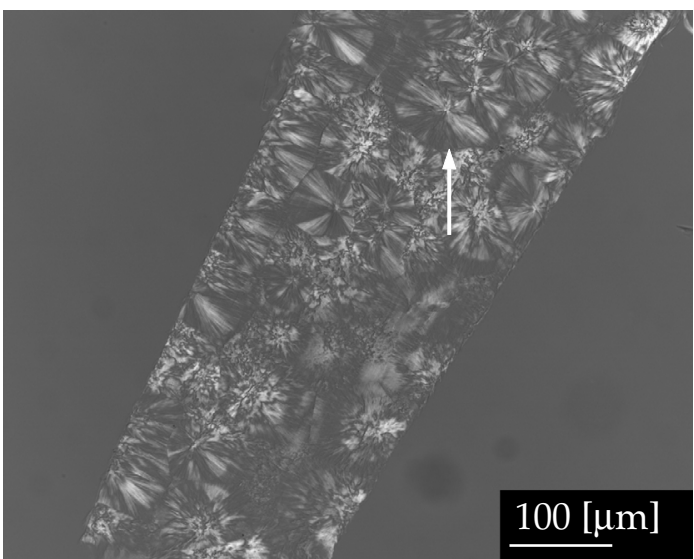

(a)

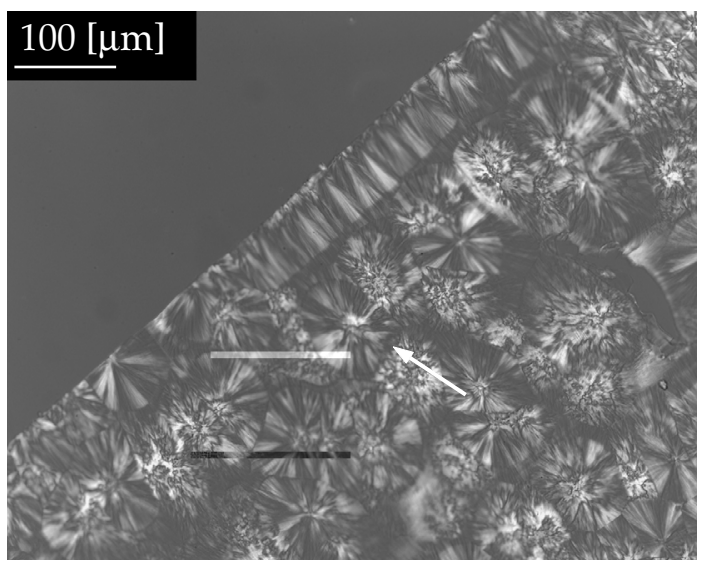

(c)

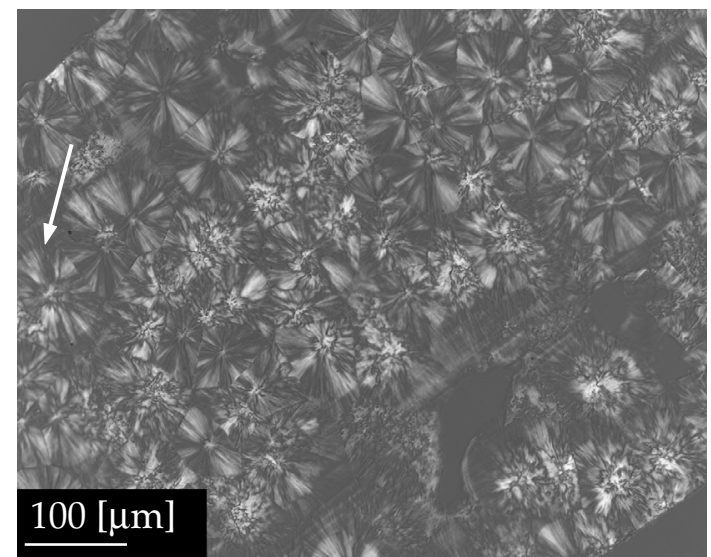

(b)

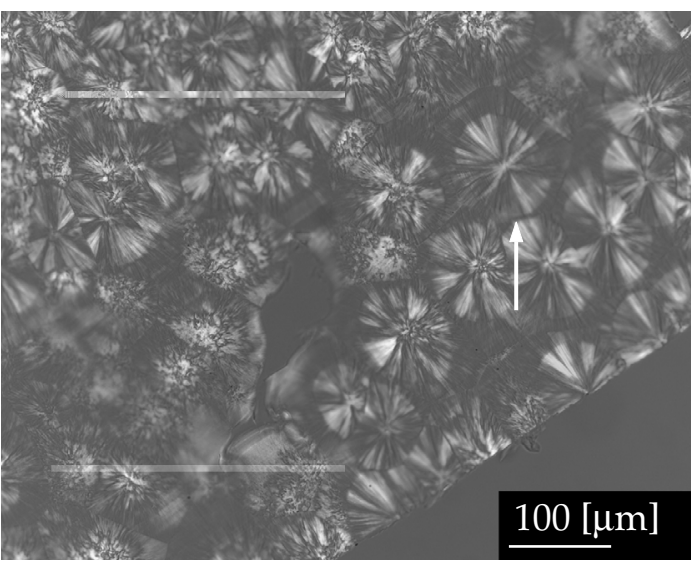

(d)

Figure 4.27: DSM13E10 after quiescent crystallization at $140\left[{ }^{\circ} \mathrm{C}\right]$. The radial position in the sample increases from (a) to (c) (close to outside and plate) or (d) (close to outside and cone). Maximum radius of spherulites that were not impinged at the end of the measurement (marked with arrows) increases only slightly with radial position: (a) $50[\mu \mathrm{m}]$; (b) $48[\mu \mathrm{m}]$; (c) $53[\mu \mathrm{m}]$ and (d) $55[\mu \mathrm{m}]$.

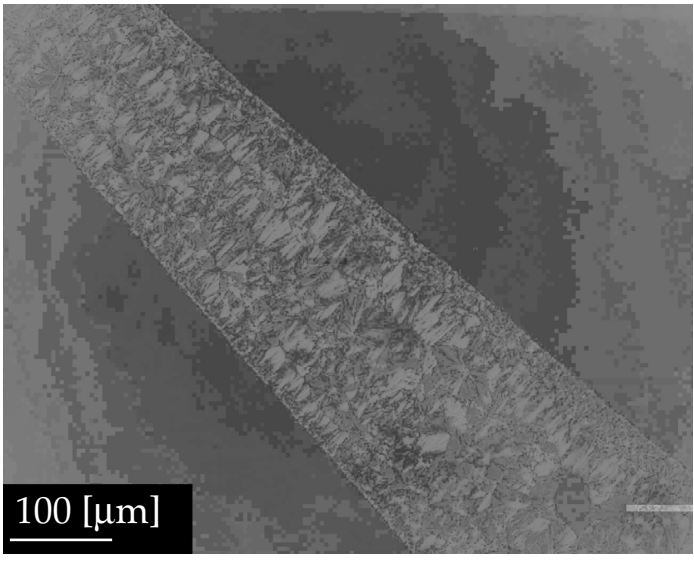

(a)

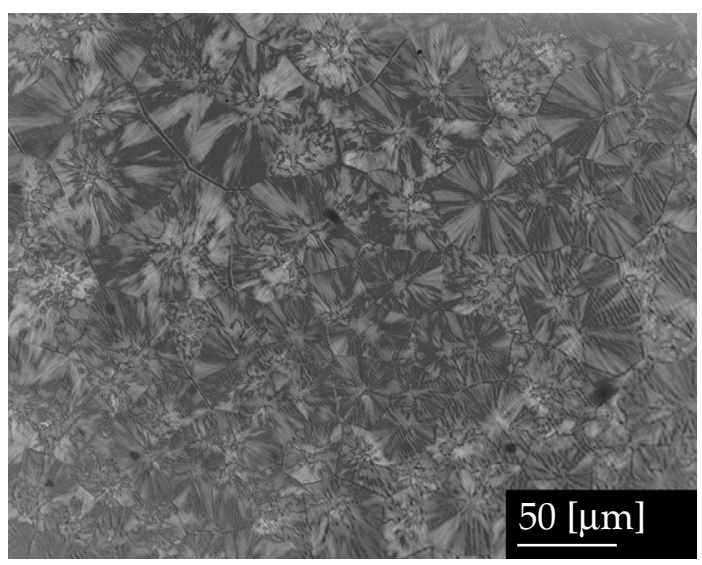

(b)

Figure 4.28: DSM13E10 after short time shearing at $140\left[{ }^{\circ} \mathrm{C}\right]$, using a shear rate of $5\left[\mathrm{~s}^{-1}\right]$ for 1 [s]. (a) Cross section perpendicular to flow direction. Notice the ordered nucleation. Contrast was enhanced using histogram equalization in Matlab (The Mathworks, Inc.). (b) Cross section in flow direction. The average radius of the spherulites is about $30[\mu \mathrm{m}]$. 
(with the two simplifying assumptions), see Figure 4.31. The spreading of the curves that can be obtained by $\beta$, introduces a slower startup behavior and a stronger dependence on the second invariant (Figure 4.29(a)). The model needs some adjustments to obtain a better quantitative description for these measurements, below the melting temperature. Consequently, the predictions on flow-induced crystallization in the remaining part of this thesis are determined for Daplen KS10, and are meant only qualitatively.

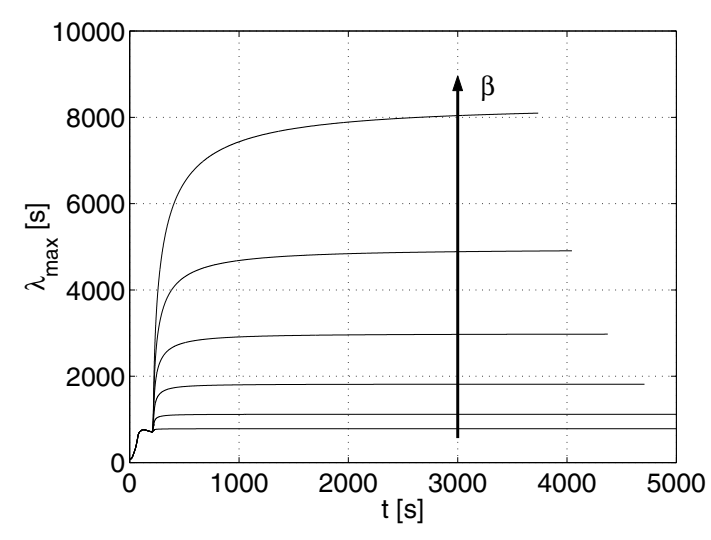

(a)

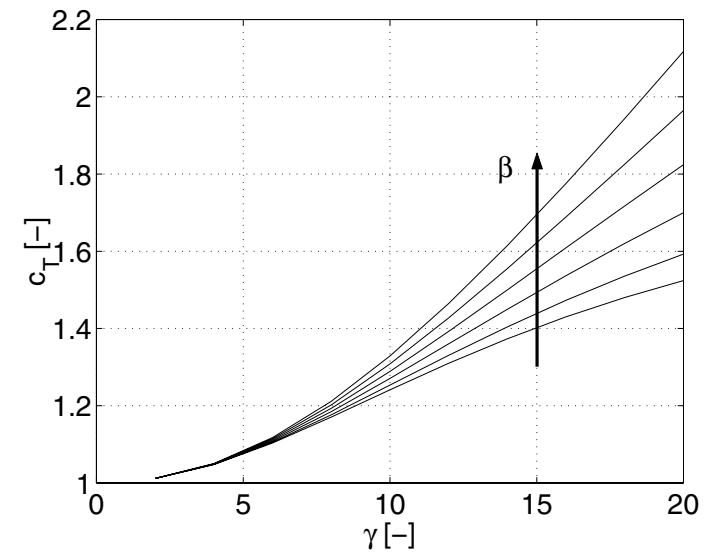

(b)

Figure 4.29: Parameter variation of $\beta$ for a constant $g_{n}^{\prime}\left(=3.12 \cdot 10^{7}\left[\mathrm{~m}^{-3} \mathrm{~s}^{-1}\right]\right)$ for DSM13E10 applying a shearrate of $1\left[\mathrm{~s}^{-1}\right]$ for $10[\mathrm{~s}] . \beta=[1,4,8,12,16,20] \cdot 10^{-7}\left[\mathrm{~s}^{-1}\right]$. (a) maximum relaxation time $\lambda_{\max }$ and (b) crystallization shift factor $\mathrm{c}_{\mathrm{T}}$.

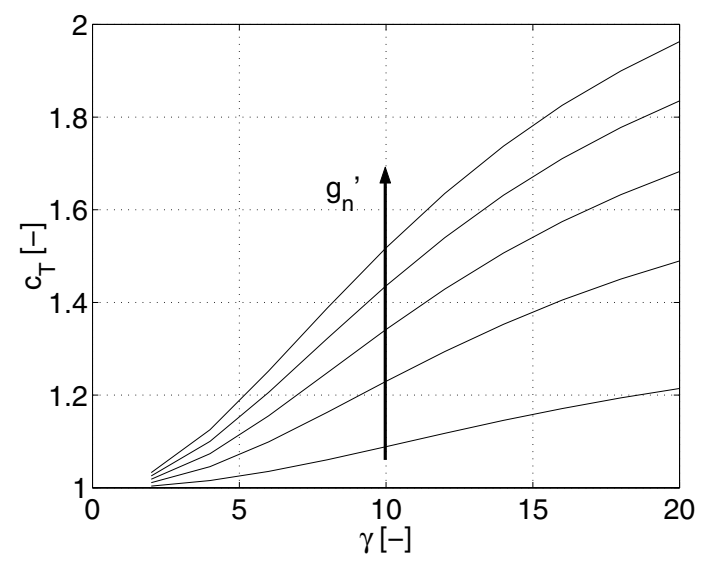

Figure 4.30: Influence of $g_{n}^{\prime}$ on the crystallization shift factor $c_{T}$ for a constant $\beta\left(=0\left[\mathrm{~s}^{-1}\right]\right)$ for DSM13E10 applying a shearrate of $1\left[\mathrm{~s}^{-1}\right]$ for $10[\mathrm{~s}] \cdot \mathrm{g}_{\mathrm{n}}^{\prime}=[1,3,5,7,9] \cdot 10^{7}\left[\mathrm{~m}^{-3} \mathrm{~s}^{-1}\right]$.

\subsubsection{Influence of nucleation on rheology}




\begin{tabular}{|c|c|c|c|c|}
\hline \multirow{2}{*}{ WLF } & & \multicolumn{2}{|c|}{$\mathrm{C}_{1}[-]$} & $\overline{\mathrm{c}_{2}[\mathrm{~K}]}$ \\
\hline & $\mathrm{a}_{\mathrm{T}}[-]$ & \multicolumn{2}{|c|}{$1.0 \cdot 10^{1}$} & $1.0 \cdot 10^{3}$ \\
\hline \multirow{7}{*}{ Maxwell } & mode & \multicolumn{2}{|c|}{ 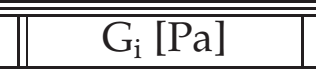 } & 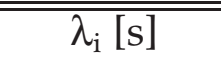 \\
\hline & 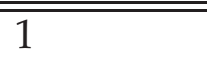 & \multicolumn{2}{|c|}{ 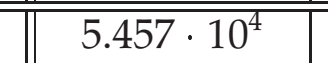 } & $8.0 \cdot 10^{-3}$ \\
\hline & 2 & \multicolumn{2}{|c|}{$1.210 \cdot 10^{4}$} & $1.144 \cdot 10^{-1}$ \\
\hline & 3 & \multirow{2}{*}{\multicolumn{2}{|c|}{$\begin{array}{c}1.208 \cdot 10^{3} \\
3.3 \cdot 10^{1}\end{array}$}} & $1.568 \cdot 10^{0}$ \\
\hline & 4 & & & $2.974 \cdot 10^{1}$ \\
\hline & & \multicolumn{2}{|c|}{$\eta_{\text {tot }}[\mathrm{Pa} \mathrm{s}]$} & $\overline{\bar{\lambda}}[\mathrm{s}]$ \\
\hline & & \multicolumn{2}{|c|}{$4.696 \cdot 10^{3}$} & $6.883 \cdot 10^{0}$ \\
\hline \multirow{2}{*}{ Carreau-Yasuda $\left(150\left[{ }^{\circ} \mathrm{C}\right]\right)$} & $\eta_{0}[\mathrm{~Pa} \mathrm{~s}]$ & \multicolumn{2}{|l|}{$\overline{l_{\mathrm{cy}}[\mathrm{s}]}$} & $\overline{\mathrm{a}[-]}$ \\
\hline & $1.658 \cdot 10^{4}$ & 1.118 & 0.3590 & 0.4911 \\
\hline \multirow{2}{*}{ Carreau-Yasuda $\left(200\left[{ }^{\circ} \mathrm{C}\right]\right)$} & $\eta_{0}[\mathrm{~Pa} \mathrm{~s}]$ & 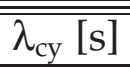 & n [-] & a [-] \\
\hline & $4.894 \cdot 10^{3}$ & 0.3301 & 0.3590 & 0.4911 \\
\hline \multirow{2}{*}{ Nucleation } & $\mathrm{c}_{\mathrm{N}}\left[\mathrm{K}^{-1}\right]$ & \multicolumn{2}{|c|}{$\mathrm{N}_{\mathrm{ref}}\left[\mathrm{m}^{-3}\right]$} & $\mathrm{T}_{\text {cref }}\left[{ }^{\circ} \mathrm{C}\right]$ \\
\hline & 0.266 & \multicolumn{2}{|c|}{$7 \cdot 7 \cdot 10^{12}$} & 1110 \\
\hline \multirow{2}{*}{ Growth } & $\mathrm{c}_{\mathrm{G}}\left[\mathrm{K}^{-2}\right]$ & \multicolumn{2}{|c|}{$\mathrm{G}_{\mathrm{ref}}[\mathrm{m} / \mathrm{s}]$} & $\mathrm{T}_{\text {cref }}\left[{ }^{\circ} \mathrm{C}\right]$ \\
\hline & $2.3 \cdot 10^{-3}$ & \multicolumn{2}{|c|}{$5.012 \cdot 10^{-6}$} & 90 \\
\hline \multirow{2}{*}{$\mathrm{S}_{\mathrm{J} 2}$} & $\mathrm{~g}_{\mathrm{n}}^{\prime}\left[\mathrm{m}^{-3} \mathrm{~s}^{-1}\right]$ & \multicolumn{2}{|c|}{$\mathrm{g}_{\mathrm{l}}^{\prime}[\mathrm{m} / \mathrm{s}]$} & $\beta\left[\mathrm{s}^{-1}\right]$ \\
\hline & $2.017 \cdot 10^{11}$ & \multicolumn{2}{|c|}{$1.429 \cdot 10^{-1}$} & $7 \cdot 10^{-6}$ \\
\hline
\end{tabular}

Table 4.7: Parameters of the fits for the WLF equation, the Maxwell and the Carreau-Yasuda model $\left(\mathrm{T}_{\mathrm{ref}}=200\left[{ }^{\circ} \mathrm{C}\right]\right)$, the fits for the number of nuclei and growth rate, and the parameters for the $\mathrm{S}_{\mathrm{J} 2}$ moduli in case of Daplen KS10. Notice that a quadratic exponential function was used to describe the growth rate. Data were taken from Zuidema [156, Appendix A]. Original data was from Eder and Janeschitz-Kriegl [35] and Jerschow [65]. The $\mathrm{S}_{\mathrm{J} 2}$ data was fitted by Zuidema [156]. 


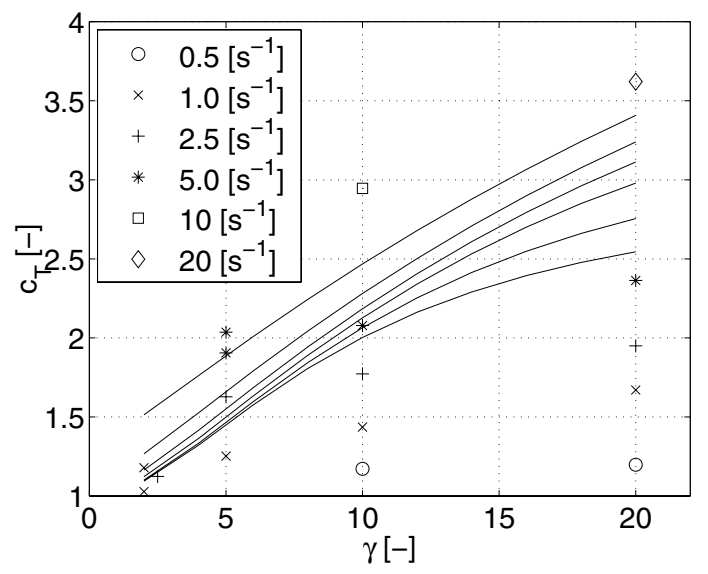

Figure 4.31: Prediction of the $S_{\mathrm{J} 2}$ for six different shear rates in the small oscilatory shear experiments on DSM13E10 (see text for the assumptions). $\beta=0\left[\mathrm{~s}^{-1}\right]$ and $\mathrm{g}_{\mathrm{n}}^{\prime}=3 \cdot 10^{8}$ $\left[\mathrm{m}^{-3} \mathrm{~s}^{-1}\right]$. Symbols: experiments; full lines: predictions.

weight distribution are equal to $235[\mathrm{~kg} / \mathrm{mol}]$ and $3.1[-]$, respectively (Jerschow and Janeschitz-Kriegl [66]).

The number of nuclei are much lower than for the two materials described in Chapter 4 . This results in a much slower quiescent crystallization behavior. For example, it takes more than 11.5 hours (41.880 [s]) to obtain a space filling of 0.95 [-] at a temperature of $140\left[{ }^{\circ} \mathrm{C}\right]$. The zero shear viscosity is of the same order as for DSM15M10, and the average relaxation time is slightly higher. At a temperature of $220\left[{ }^{\circ} \mathrm{C}\right]$, these are equal to 3116 [Pa.s] and 4.38 [s], respectively, using a shift factor of 0.637 [-].

The influence of nucleation on rheology predicted by the $S_{\mathrm{J}_{2}}$ model is investigated for a range of shear and elongational rates. The influence on subsequent crystallization is not discussed.

The numerical experiment consisted of the following three steps. First, the polypropylene melt (Daplen $\mathrm{KS} 10$ ) is cooled from $240\left[{ }^{\circ} \mathrm{C}\right]$ to the flow temperature of $170\left[{ }^{\circ} \mathrm{C}\right]$, using a cooling rate of $65\left[{ }^{\circ} \mathrm{C} / \mathrm{min}\right]$. Next, flow was applied for 500 [s] using shear and elongational rates in a range of $0.01-100\left[\mathrm{~s}^{-1}\right]$ (flow starts at $\mathrm{t}=0[\mathrm{~s}]$ ). This long flow time was chosen to reach the steady viscosity also for the lower rates investigated. Finally, the relaxation behavior is monitored.

Parameter $\beta$ describes the dependence of the largest relaxation time on the number of flow-induced nuclei. Introduction of this parameter will increase the relaxation time during flow, but two situations can be distinguised after flow. If the shear rate was low, the maximum relaxation time wil decrease to its original value, due to the disappearance of the number of nuclei (Figure 4.32). If the rate was 'high', the maximum relaxation time reaches a constant level or increases even further. Due to this behavior in elongational flow, the integration scheme becomes unstable for rates larger than $0.17\left[\mathrm{~s}^{-1}\right]$. Therefore, the $S_{\mathrm{J} 2}$ model needs some adjustments to obtain a more realistic value for the relaxation time.

The 'steady state' shear viscosity value in shear at $t=500$ [s] is not influenced by the increasing value of the maximum relaxation time, while an upswing is observed in the first normal stress difference at $t=500$ [s] for shear rates larger than $15\left[\mathrm{~s}^{-1}\right]$. After 


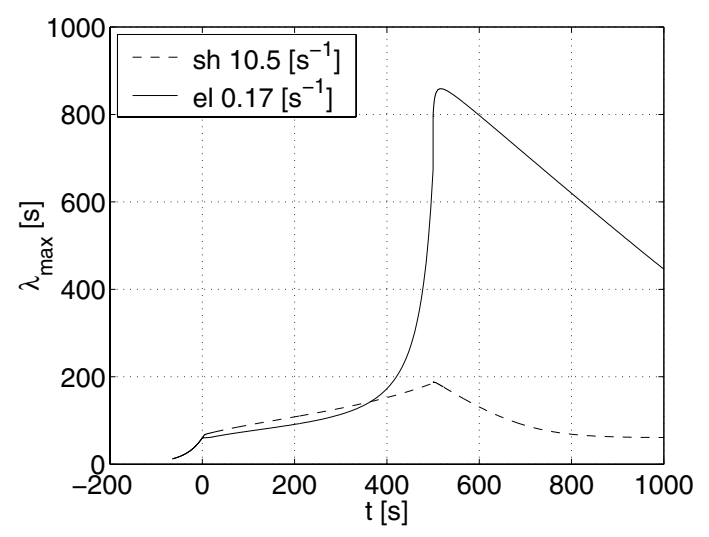

Figure 4.32: Increase of the maximum relaxation time according to the $\mathrm{S}_{\mathrm{J} 2}$ model in case of a shear rate of $10.5\left[\mathrm{~s}^{-1}\right]$ or an elongational rate of $0.17\left[\mathrm{~s}^{-1}\right]$, for Daplen KS10 at $170\left[{ }^{\circ} \mathrm{C}\right]$.
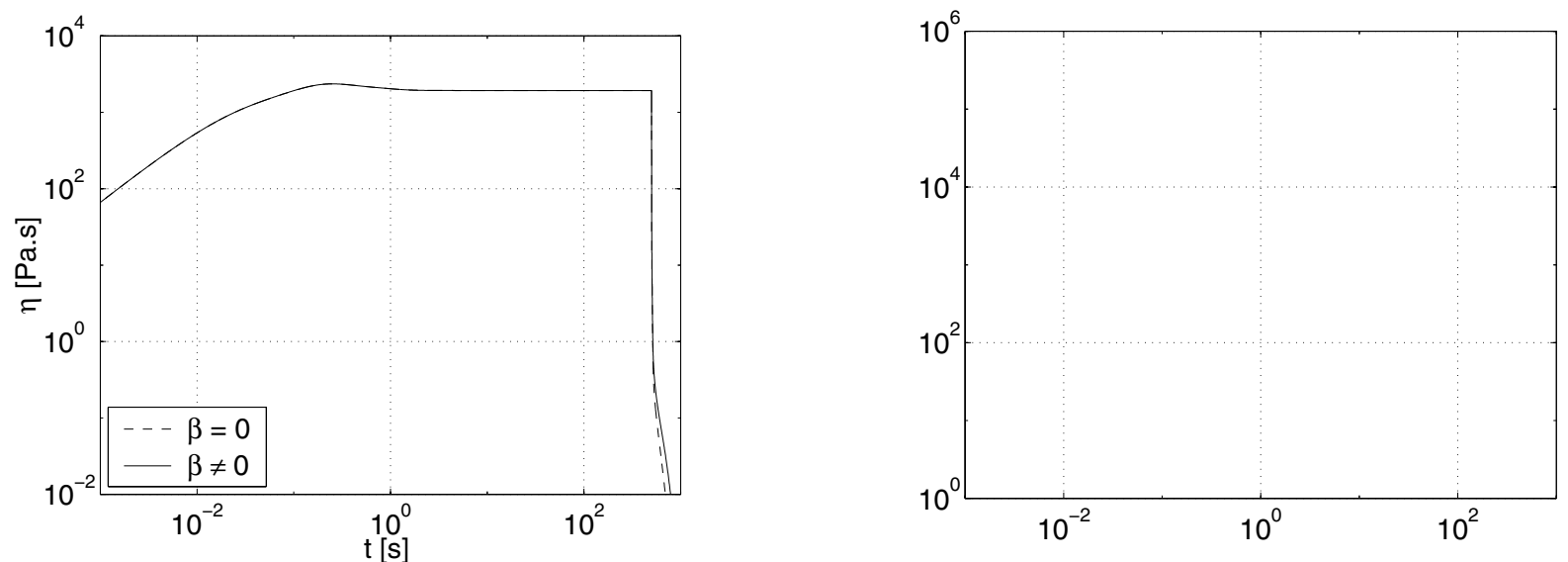

(a) 
cessation of shear flow, both viscosity and first normal stress difference show a slower relaxation at $10\left[\mathrm{~s}^{-1}\right]$, a shear rate at which the steady state values were not influenced (Figure 4.33). The experimental observations in Chapter 2, no changes in startup behavior and small changes during relaxation, agree qualitatively with the model predictions.

Similar numerical observations are predicted for planar elongation flow, in which two viscosities can be defined

$$
\eta_{e p 1}=\frac{\tau_{11}-\tau_{22}}{\dot{\varepsilon}} \quad \text { and } \quad \eta_{e p 2}=\frac{\tau_{33}-\tau_{22}}{\dot{\varepsilon}}
$$

with the first and second planar viscosities $\eta_{\mathrm{ep} 1}$ and $\eta_{\mathrm{ep} 2}$, respectively. The flow in the center of the cross-slot flow cell can be considered as a planar elongational flow. Changes during flow are only observed for $\eta_{\text {ep } 1}$, while relaxation behavior of both viscosities is changed after flow (Figure 4.34).

\subsection{Discussion}

Rheological and crystallization characterization are performed for two isotactic polypropylenes, DSM13E10 and DSM15M10. The larger molecular weight of DSM13E10 results in larger relaxation times, larger viscosities and longer relaxation times. It was already postulated in Chapter 1 that this last characteristic is important in stressinduced crystallization. In quiesent crystallization, the growth rates for both materials are about the same, but much more nuclei are found in DSM13E10, resulting in faster crystallization in DSC and the rheometer experiments.

Modulus versus space filling was compared for several isothermal quiescent crystallization experiments, showing no unique relation for both materials. It seems that this relation is very sensitive to experimental errors. In case of DSM13E10, the two shift factors for $\mathrm{G}^{\prime}$, which are slightly above the Schneider shift factors (at 138 and $144\left[{ }^{\circ} \mathrm{C}\right]$; Table 4.6), show a more sensitive dependence of space filling on the modulus (Figure 4.24). This faster increase in the modulus can be explained by the transcrystallization layer observed in light microscopy. The influence of this layer can be twofold. First, it increases the amount of nuclei in the sample. Second, a more sensitive behavior will be observed, if the thickness of this layer is of the same order of magnitude as the gap height for the cone-plate geometry. Therefore, careful attention should be paid to measurements at high crystallization temperatures for materials with a low amount of bulk nuclei (i.e. DSM15M10). It is recommended to investigate this explanation, by comparing with similar experiments in a plate-plate geometry.

It was not possible to obtain a good quantitative fit for the crystallization shift factor $\mathrm{c}_{\mathrm{T}}$ obtained from the small oscillatory shear experiments. The main problem is to describe the startup behavior of the experiments. The development of orientation during the start of the experiments is much more pronounced than can be described by the $S_{\mathrm{J} 2}$ model. It is recommended to investigate other stress measures that might be able to give a better quantitative relation between crystallization kinetics and the deformation history applied. 



\section{Chapter 5}

\section{Nucleation and crystallization in a cross-slot flow cell: Numerical analysis}

\subsection{Introduction}

A cross-slot flow cell is used to investigate the crystallization behavior of isotactic polypropylene in a complex flow with a strong elongational component. In this chapter, the behavior of the constitutive models in the cross-slot flow is discussed.

Calculations were done for two materials, DSM13E10 and Daplen KS10. However, the parameter sets for both materials were incomplete. DSM13E10 is characterized rheologically both in shear and elongation, but the $\mathrm{S}_{\mathrm{J} 2}$ parameters are unknown. It is, therefore, used to compare the stresses predicted by the viscoelastic models, and to show qualitatively, the influence on flow-induced crystallization by using estimated parameter values for the $S_{\mathrm{J} 2}$ model. The elongational behavior of Daplen KS10 could not be characterized, but the $S_{\text {J2 }}$ parameters are available. Therefore, this material is used to show the behavior of the $S_{\mathrm{J} 2}$ model in the cross-slot flow, in more detail.

The schematic of the cross-slot flow cell together with its dimensions, are given in Figure 5.1. It consists of two curved reservoirs and a cross-slot. Flow is created by rotating two cams which are connected to the outer ring. A stagnation line will occur in the center of the cross-slot. Depth of the channels and reservoirs is 2.0 [mm]. In Figure 5.1, also the parameter values are given to obtain a strain rate in the center of about $10\left[\mathrm{~s}^{-1}\right]$. The corresponding flow rate in one inflow or outflow channel is equal to $1.85 \cdot 10^{-8}\left[\mathrm{~m}^{3} / \mathrm{s}\right]$. The total maximum strain of particles travelling close to the stagnation line is about 10.6 [-].

Numerical methods and results for the determination of the stresses and space filling values in the cross-slot flow cell are described in Section 5.2 and 5.3, respectively.

\subsection{Numerical methods}

Since three-dimensional finite or spectral element calculations with non-linear viscoelastic models are expensive and time consuming, the calculation of the velocities and stresses are performed in a decoupled way (Douven et al. [31]). The velocity field 


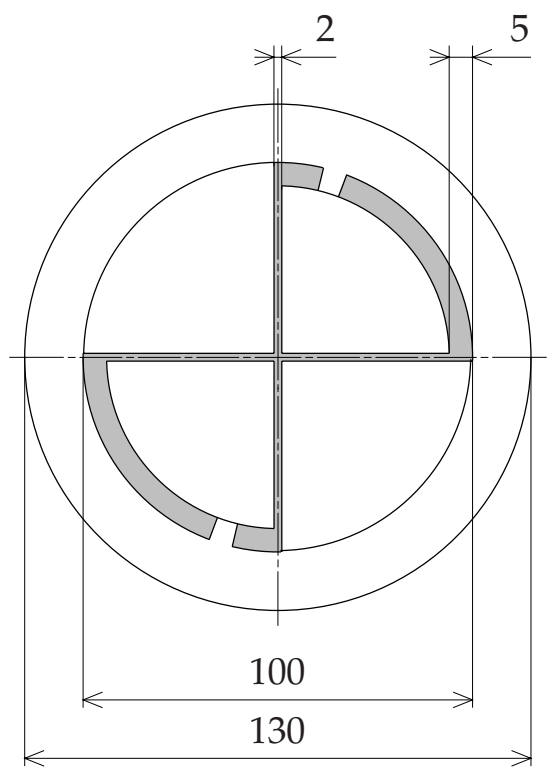

\begin{tabular}{|l|r|}
\hline \multicolumn{1}{|c|}{ Parameter } & \multicolumn{1}{c|}{ Value } \\
\hline \hline $\mathrm{v}_{\text {mean } 3 \mathrm{D}}[\mathrm{m} / \mathrm{s}]$ & $4.63 \cdot 10^{-3}$ \\
\hline $\mathrm{Q}\left[\mathrm{m}^{3} / \mathrm{s}\right]$ & $1.85 \cdot 10^{-8}$ \\
\hline $\mathrm{t}[\mathrm{s}]$ & 40 \\
\hline$\dot{\varepsilon}\left[\mathrm{s}^{-1}\right]$ & 10.4 \\
\hline$\varepsilon[-]$ & 10.6 \\
\hline
\end{tabular}

Figure 5.1: Dimensions in [mm] (left) and flow specifications (right) of the cross-slot flow cell to reach an elongational rate $\dot{\varepsilon}$ of about $10\left[\mathrm{~s}^{-1}\right] . \mathrm{v}_{\text {mean } 3 \mathrm{D}}$ : three-dimensional mean velocity in one inflow channel; $Q$ : flow rate in one inflow channel; $t$ : corresponding time for a full stroke of the ring; $\varepsilon$ : elongational strain.

is computed with the finite element package SEPRAN (Segal [133]), using the generalized Newtonian Carreau-Yasuda model as the constitutive equation for the stresses. Particle paths and velocity gradients determined from the velocity field are used to calculate the three-dimensional stresses predicted by the non-linear viscoelastic models. Four different numerical aspects can be distinguished to test the behavior of the models in the cross-slot flow cell:

1. Computation of the three-dimensional velocity field.

2. Determination of the particle paths (particle tracking).

3. Interpolation of the velocity gradient tensor $\mathbf{L}$.

4. Calculation of stress related properties.

\section{ad 1. Computation of the three-dimensional velocity field}

The dimensionless form of the Navier-Stokes equations (without a characteristic force) and the equation for conservation of mass are given by

$$
\begin{aligned}
\text { St } \frac{\partial \boldsymbol{u}}{\partial t}+\boldsymbol{u} \cdot \nabla \boldsymbol{u} & =-\nabla p+\frac{1}{\operatorname{Re}} \nabla \cdot 2 \eta \boldsymbol{D} \\
\nabla \cdot \boldsymbol{u} & =0
\end{aligned}
$$

in which the Strouhal number $(\mathrm{St}=\mathrm{L} \omega / \mathrm{U})$ is the ratio between instationary and stationary inertia forces, and the Reynolds number $(\operatorname{Re}=\rho U L / \eta)$ the ratio between stationary inertia forces versus viscous forces. A time marching scheme is used to solve Equation (5.1), giving the solution for the stationary Stokes equations for small Reynolds 
numbers $(\operatorname{Re}<<1)$. The position and velocity in the cross-slot flow are scaled with $1[\mathrm{~mm}](\mathrm{L})$ and $1[\mathrm{~mm} / \mathrm{s}](\mathrm{U})$. Consequently, the dimensionless flow rate, which is used to determine the inflow boundary condition, is given by $\mathrm{Q} /\left(\mathrm{L}^{2} \mathrm{U}\right)$.

Given the basic dimensions of the cell and the typical flow rates used, the nonlinear convection term $(\mathbf{u} \cdot \nabla \mathbf{u}$ in Equation (5.1)) can be neglected and the stationary Stokes equations are solved to obtain the velocity field. A finite element preconditioner in combination with a conjugate gradient iterative solver is applied in order to reduce computational costs. The preconditioner increases the efficiency of spectral element methods with iterative solvers, and therefore decreases the number of iterations (Anderson [4]). Details about the space and time discretization can be found in this reference.

Only the inner part of the cross was modeled to minimalize computional cost and use all computational capacity to obtain a high accuracy for the velocity. Moreover, due to symmetry, only one eight of the cell is modeled. The problem definition is given in Figure 5.2(a). The Cartesian coordinates $(x, y, z)$ are defined in outflow, inflow and neutral (i.e. viewing) direction, respectively. The stagnation line is defined as $(0,0, \mathrm{z})$. The inflow and outflow 'centerline' are defined as $(0, y, z)$ and $(x, 0, z)$, respectively. The essential boundary conditions for the velocity components $(u, v, w)$ and the pressure $(p)$ are defined on surfaces:

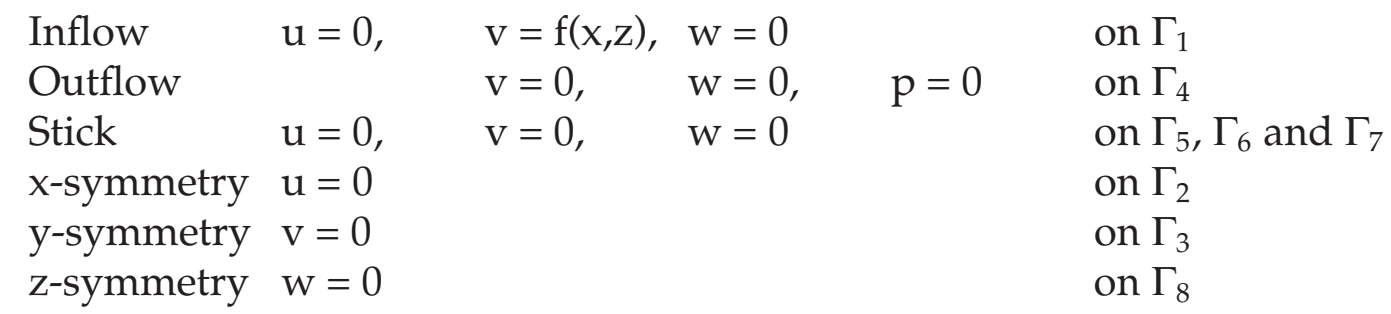

with function $\mathrm{f}(\mathrm{x}, \mathrm{z})$ a Carreau-Yasuda flow profile in a rectangular duct. The boundary condition for the pressure is only defined on the outflow surface $\left(\Gamma_{4}\right)$.

Since, there is no analytical description of a Carreau-Yasuda flow in a three-dimensional, rectangular channel, the inflow boundary condition of the cross-slot flow is determined by calculating the velocity field for the 'Carreau-Yasuda fluid' in a rectangular channel first. The length, depth and height of the corresponding mesh were equal to $4.0 \times 1.0 \times 1.0\left[\mathrm{~mm}^{3}\right]$. The spectral element mesh consists of 20 , eight order (i.e. seven intermediate points) elements, giving 11.849 nodal points. A fully developed Newtonian inflow boundary condition was used for this calculation (see White [150]). Similar outflow, stick and symmetry boundary conditions as for the cross-slot were applied. The calculated outflow surface was taken as inflow boundary condition for the cross-slot flow.

The finite element representation of the spectral element mesh for the cross-slot flow is shown in Figure 5.2(b). The spectral element mesh consists of 32, eight order elements giving 18.785 nodal points. Mesh refinement was used in $\mathrm{x}$ - and $\mathrm{y}$-direction towards the corner. The inflow and outflow length are equal to $2.5[\mathrm{~mm}]$ and the height and width of the mesh are both equal to $1.0[\mathrm{~mm}]$. 


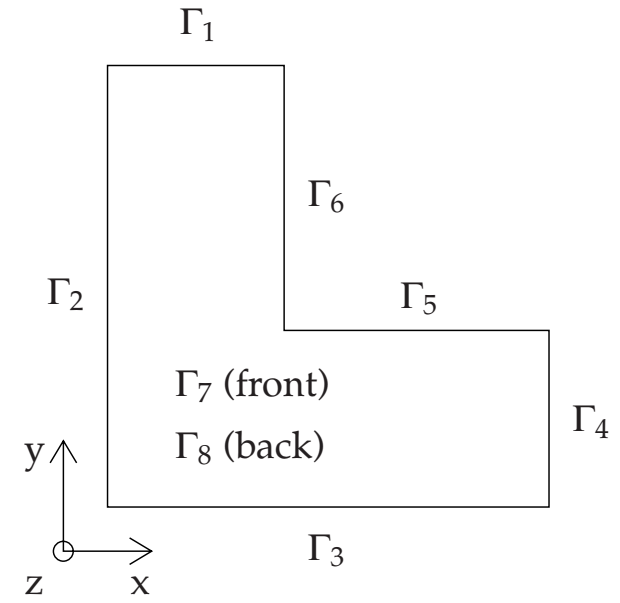

(a)

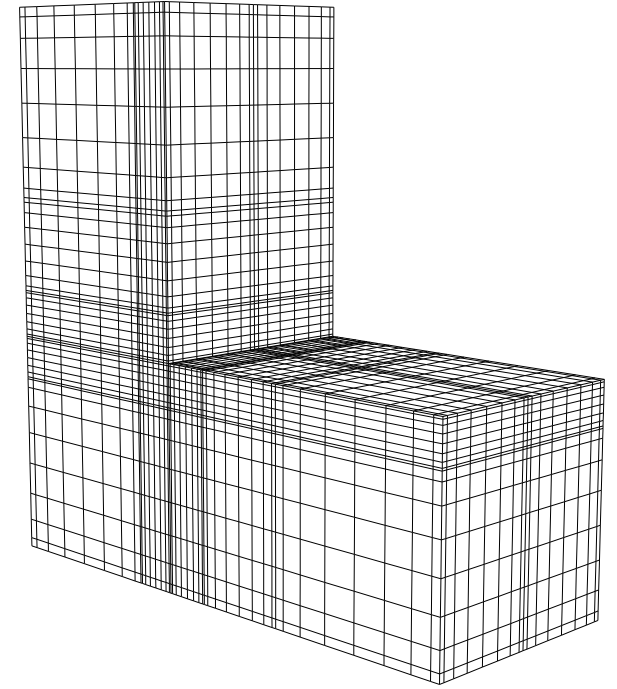

(b)

Figure 5.2: (a) Problem definition for the cross-slot flow. Surface boundary conditions: Carreau-Yasuda inflow profile on $\Gamma_{1}$, outflow on $\Gamma_{4}$, stick condition on $\Gamma_{5}, \Gamma_{6}$ and $\Gamma_{7}$, x-symmetry on $\Gamma_{2}$, y-symmetry on $\Gamma_{3}$, z-symmetry on $\Gamma_{8}$. (b) Finite element representation of the spectral element mesh.

\section{ad 2. Determination of the particle paths (particle tracking)}

Determination of the particle paths is needed to determine the deformation history for some user defined points. Backward particle tracking is performed on the velocity field (i.e. particle tracking on $-\mathbf{u}$ ). Particle positions are determined for constant time intervals with a first order Euler method.

Limitation of the three-dimensional particle tracking is that the starting position should not be on the edge of the grid, otherwise particles can leave the mesh at other surfaces than the inflow surface due to rounding-off errors.

In the calculations, the complete history during a user defined time is taken into account. In case the residence time of the particle in the cross-slot mesh is smaller than the deformation time, the deformation history was extended. Two choices for this extended history can be made, namely applying the velocity gradients at the entrance of the cross-slot mesh or applying the velocity gradients of the rectangular slit, in a well developed flow region, both for the remaining time. As the divergence or the solution error in the cross-slot is larger (due to the singular corner), the more accurate velocity gradients in the rectangular channel were used. The velocity gradients in the rectangular channel were determined using the entrance position of the particles in the cross-slot mesh, again by interpolation using polynomial basis functions.

\section{ad 3. Interpolation of the velocity gradient tensor $\mathrm{L}$}

Due to the spatial discretization, the velocities and velocity gradients are only known at the interpolation points of the mesh. Interpolation is necessary to obtain the velocity gradients on the particle paths. Highest accuracy for this interpolation is obtained if the polynomial basis functions are used (Anderson [4]). Consequently, the interpola- 
tion error is of the same order as the error in the discretized data. Both velocities and velocity gradients are determined on the particle paths.

\section{ad 4. Calculation of stress related properties}

Finally, stresses and stress related properties are calculated. All modes of the time relaxation spectrum were used to determine the stresses, while only the highest relaxation time is used for the calculation of the second invariant of the deviatoric part of the elastic Finger tensor. A first order implicit Euler scheme is used to determine the stress, the quiescent part $\left(\phi_{\mathrm{i}}\right)$ and the flow-induced part $\left(\psi_{\mathrm{i}}\right)$ of the crystallization. Different time steps are chosen for the flow, the (non-)isothermal relaxation, and crystallization part of the calculation. Small time steps of 0.01 [s] are used during flow. After cessation of flow, the time step is increased logarithmically to a value of 1 [s] in a period of 100 [s] (900 points). This time step is also used in the remaining part of the calculation at the crystallization temperature for about 30 minutes.

The influence of an undershoot in temperature was taken into account by keeping all nuclei that were created at the lowest temperature, while the growth rate was dependent on the instantaneous temperature.

\subsection{Numerical results}

The stresses in the cross-slot flow cell are determined for two materials according to the procedure described in the previous section. The typical response of the two viscoelastic models is demonstrated for DSM13E10. Space filling is only shown for Daplen KS10, because only for this material the flow-induced crystallization parameters are known.

The stresses and space filling are calculated in the z-symmetry plane for $x>1 \cdot 10^{-6}$ $[\mathrm{mm}]$ and $y>5 \cdot 10^{-3}[\mathrm{~mm}]$. Some extra points were defined close to the outflow centerline, because large stress gradients are expected here. A flow rate of $1.85 \cdot 10^{-8}\left[\mathrm{~m}^{3} / \mathrm{s}\right]$ (in one inflow channel) for 40 [s] was used at a temperature of $200\left[{ }^{\circ} \mathrm{C}\right]$.

The velocity gradients obtained from the finite element calculation for both materials are shown in Appendix C. Large values for the velocity gradient are found close to the corner due to the singularity at this corner. The main difference between DSM13E10 and Daplen KS10 is the larger shear thinning effect for DSM13E10.

\section{DSM13E10}

The stresses are calculated in the $z$-symmetry plane $(\mathrm{z}=0[\mathrm{~mm}])$ for 150 points, using the Leonov and Pompom model, without crystallization coupling. The most important stresses in this plane are shown in Figures 5.3-5.5. Both models give about the same prediction for the shear stress (Figure 5.3), as expected. The values close to the corner are influenced by the large gradients. The first $\left(\mathrm{N}_{1}\right)$ and second $\left(\mathrm{N}_{2}\right)$ normal stress difference are defined as

$$
N_{1}=\tau_{x x}-\tau_{y y} \quad \text { and } \quad N_{2}=\tau_{y y}-\tau_{z z}
$$




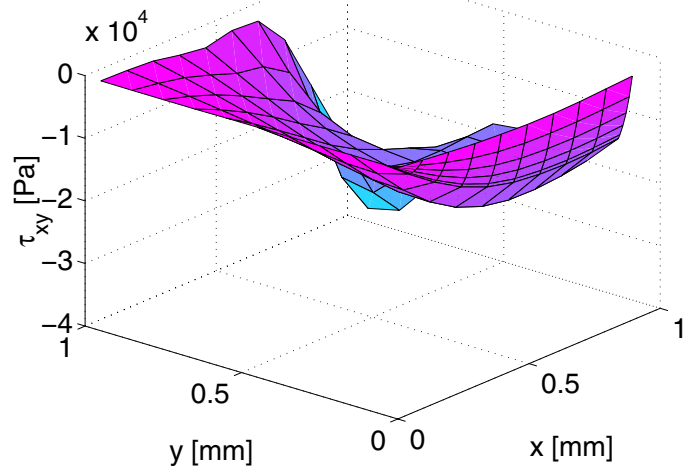

(a)

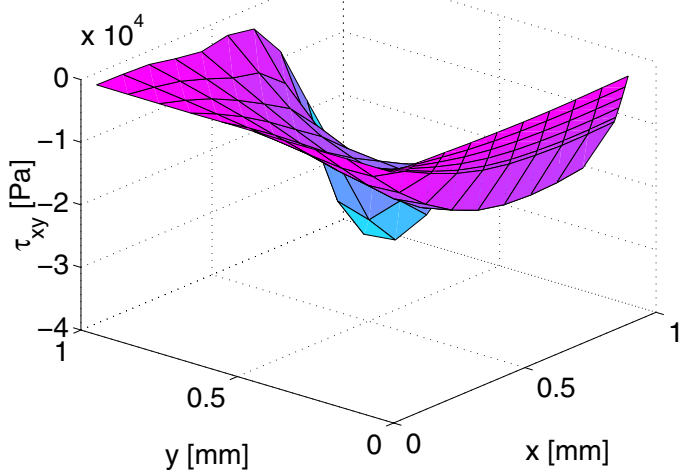

(b)

Figure 5.3: Prediction of the shear stress $\tau_{\mathrm{xy}}$ in the cross-slot flow cell for DSM13E10 at $200\left[{ }^{\circ} \mathrm{C}\right]$ after a flow rate of $1.85 \cdot 10^{-8}\left[\mathrm{~m}^{3} / \mathrm{s}\right]$ for 40 [s], without crystallization coupling $(\alpha=0)$. (a) Leonov and (b) Pompom.

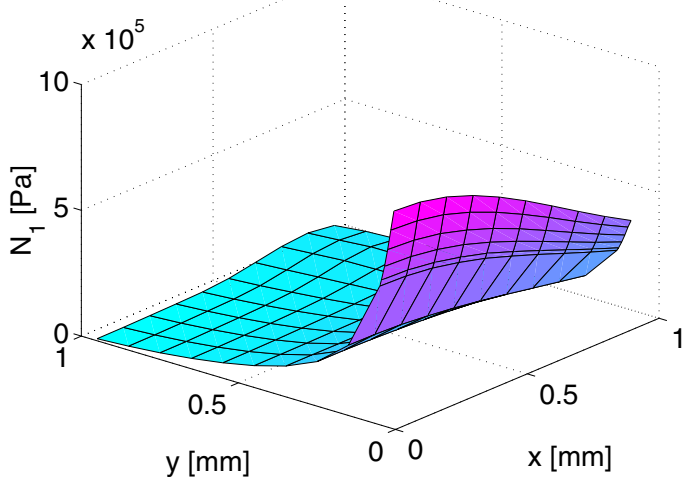

(a)

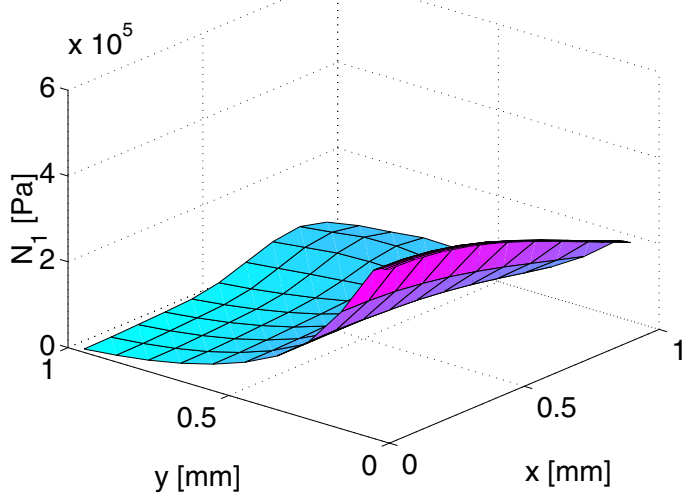

(b)

Figure 5.4: Prediction of the first normal stress difference $\left(\mathrm{N}_{1}\right)$, conditions as in Figure 5.3. (a) Leonov and (b) Pompom.

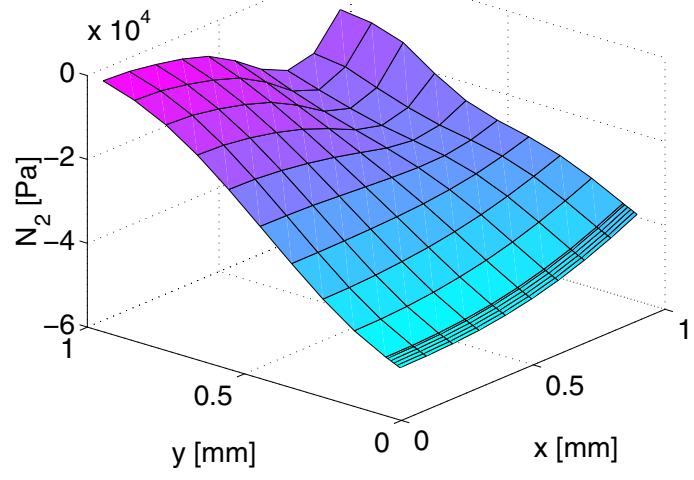

(a)

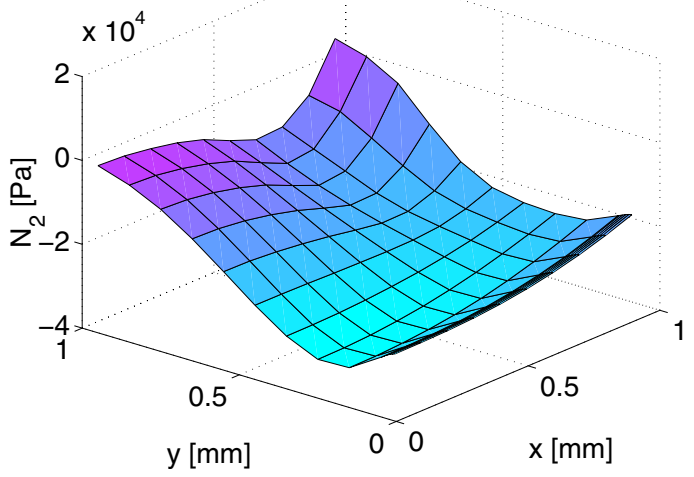

(b)

Figure 5.5: Prediction of the second normal stress difference $\left(\mathrm{N}_{2}\right)$, conditions as in Figure 5.3. (a) Leonov and (b) Pompom. 


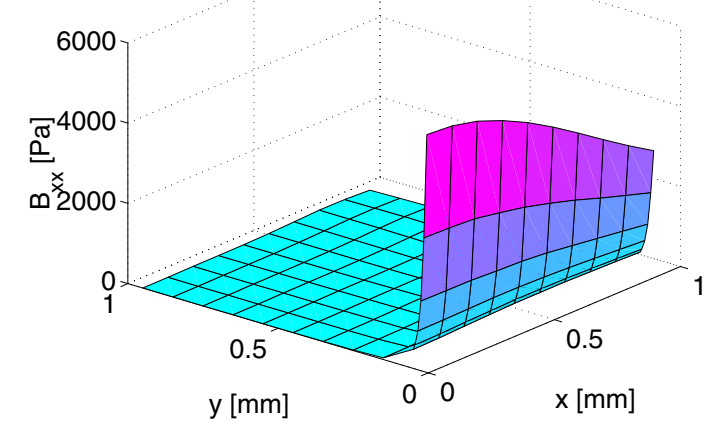

(a)

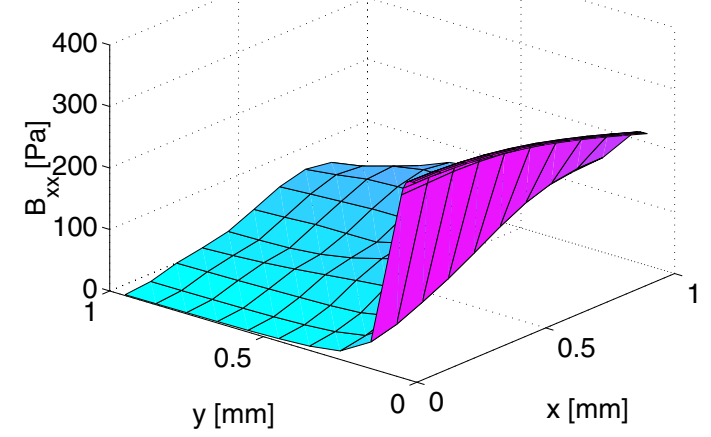

(b)

Figure 5.6: Prediction of the first component of the Finger tensor $\left(\mathrm{B}_{\mathrm{xx}}\right)$, conditions as in Figure 5.3. (a) Leonov and (b) Pompom.

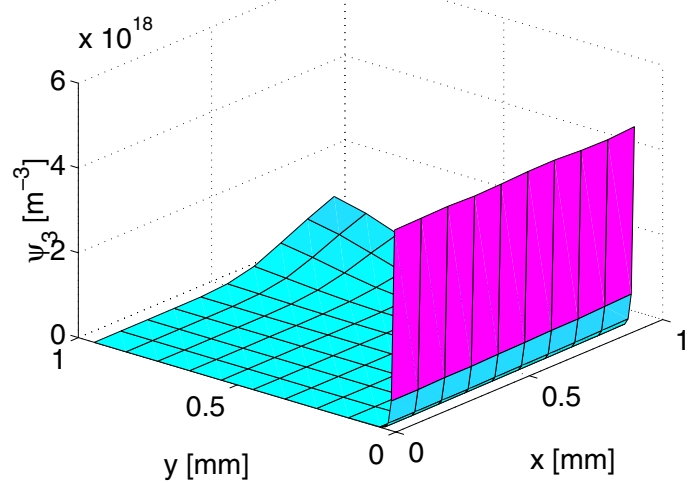

(a)

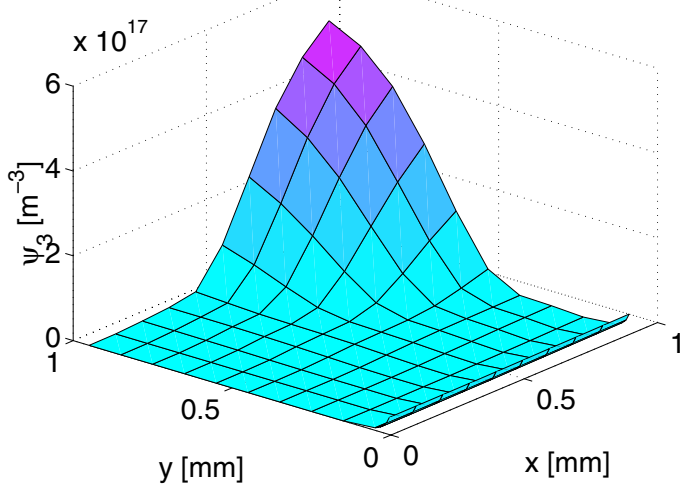

(b)

Figure 5.7: Prediction of $8 \times$ the number of flow-induced 'nuclei' $\left(\psi_{3}\right)$, conditions as in Figure 5.3. $\tau_{\mathrm{n}}$ was chosen equal to the maximum relaxation time $(\alpha=0)$ and $\mathrm{g}_{\mathrm{n}}^{\prime}$ was taken equal to $2 \cdot 017 \cdot 10^{11}\left[\mathrm{~m}^{-3} \mathrm{~s}^{-1}\right]$ (value of Daplen KS10) (a) Leonov and (b) Pompom.

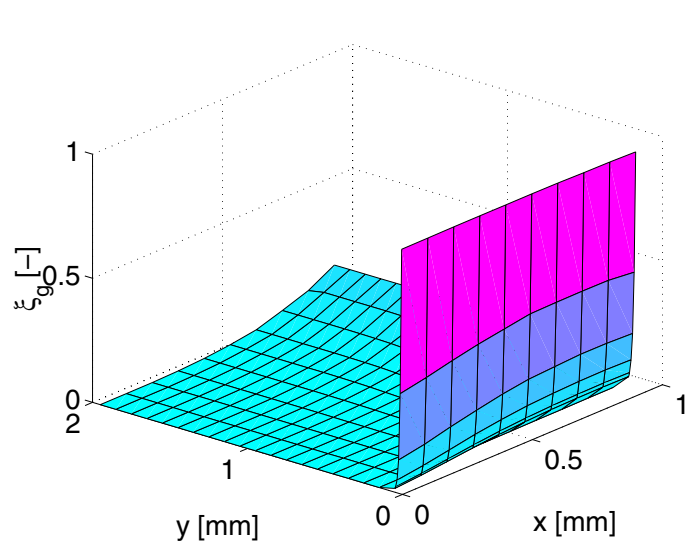

(a)

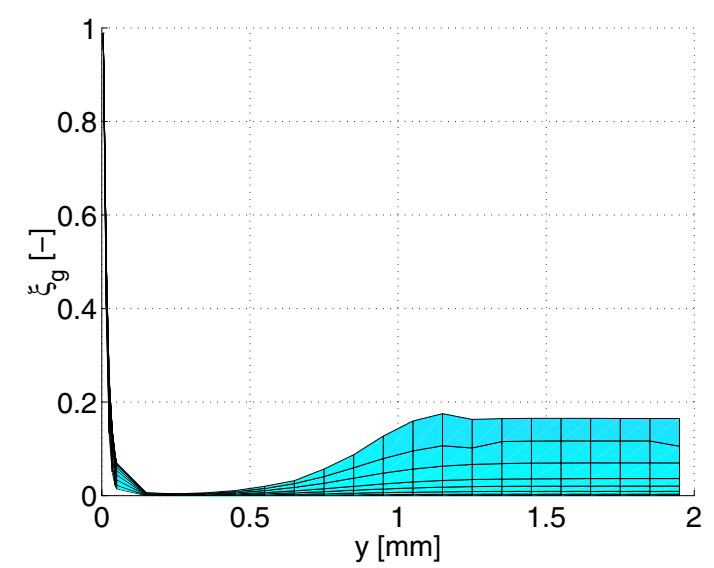

(b)

Figure 5.8: Prediction of the space filling in the cross-slot flow cell at $\mathrm{z}=0$ [mm] for Daplen KS10 after applying a flow rate of $1.85 \cdot 10^{-8}\left[\mathrm{~m}^{3} / \mathrm{s}\right]$ for 40 [s], and waiting $800[\mathrm{~s}]$ at $140\left[{ }^{\circ} \mathrm{C}\right]$ using the $\mathrm{S}_{\mathrm{J} 2}$ model. (a) 3D view (b) side view. 
and shown in Figures 5.4 and 5.5, respectively. Both models show different behavior close to the outflow centerline. Increasing values with decreasing distance from the outflow centerline are observed for the Leonov model, while the values level off for the Pompom model. The large difference between the two models for higher elongational rates was already shown when fitting the uniaxial elongation experiments (Figure 4.7).

Predicted values for both $\mathrm{N}_{1}$ and $\mathrm{N}_{2}$ are about twice as large for the Leonov model. Of course, this influences the Finger tensor and thus the resulting flow-induced nuclei, which are shown in Figures 5.6 and 5.7. It is questionable whether these nuclei really exist at this temperature, but Figure 5.7 is only presented to show the behavior of the model. It is clear that the values for $\mathrm{B}_{\mathrm{xx}}$ and $\psi_{3}$ are largely influenced by the different elongation behavior of the models.

\section{Daplen KS10}

The space filling for Daplen KS10 is calculated in the $\mathrm{z}$-symmetry plane ( $\mathrm{z}=0$ [mm]) for 250 points, using the $S_{\mathrm{J} 2}$ model. The numerical experiment consisted of the following steps: creating flow at $200\left[{ }^{\circ} \mathrm{C}\right]$, cooling to a crystallization temperature of $140\left[{ }^{\circ} \mathrm{C}\right]$ using a rate of $30\left[{ }^{\circ} \mathrm{C} / \mathrm{min}\right]$ and observing the isothermal crystallization for 30 minutes. Time $\mathrm{t}=0[\mathrm{~s}]$ is defined as the time reaching $\mathrm{T}_{\mathrm{c}}$.

The predicted space filling is shown in Figure 5.8. Large values are found close to the outflow centerline and the corner. The large gradients caused by the singularity in the corner do not have a large influence on the space filling, because the whole deformation history was used. Therefore, the local large velocity gradients are only observed as a small increase in the space filling value at $y=1.15[\mathrm{~mm}]$ (see Figure $5.8(\mathrm{~b})$ ).

A small increase in space filling is observed on the outflow centerline with increasing $\mathrm{x}$-coordinate. This was also observed experimentally by McHugh et al. [96] as discussed in Section 2.3.

\subsection{Discussion}

A spectral element method was used to determine the velocity gradient tensor in the cross-slot flow cell. An advantage of the spectral element method above the finite element method is the higher accuracy that can be obtained, due to the higher order elements. Singular points like the corner in the cross-slot can have a large influence on the solution. A small rounding in the corner can reduce the large gradients, and increase the accuracy of the solution. However, the whole history influences the crystallization behavior and, therefore, the influence of the larger gradients calculated on space filling is small.

Interesting is the intrinsic behavior of the two models used: Leonov and Pompom, without crystallization coupling. The strain hardening behavior in elongation for the Leonov model over-estimates the first component of the Finger tensor and, therefore, the number of flow-induced nuclei for the $S_{\mathrm{J} 2}$ model. 


\section{Chapter 6}

\section{Nucleation and crystallization in a cross-slot flow cell: Experiments and predictions}

\subsection{Introduction}

A cross-slot flow device for melts was designed to study structure development in a complex flow with a strong elongational component (Swartjes [137]). The flow cell is accessible for both optical measurements, like birefringence and laser light scattering, as well as for Wide and Small Angle X-ray Scattering (WAXS and SAXS). Optical measurements are performed in our laboratory in Eindhoven, while the X-ray scattering experiments are carried out in Grenoble (France) at the European Synchrotron Radiation Facility (ESRF), because a high X-ray flux is needed for time-resolved experiments. Moreover, to investigate the influence of deformation history, a small beam-size is necessary in the inhomogeneous flow field.

The experimental set-up, including the flow cell, is described in Section 6.2. Experimental results for the birefringence and X-ray experiments are discussed in Sections 6.3 and 6.4, respectively. Finally, the experimental results are qualitatively compared with numerical predictions in Section 6.5.

\subsection{Cross slot flow cell}

The basic design of the flow cell was already shown in the previous chapter (Figure 5.1). The flow cell consists of a cross-slot and two reservoirs where two cams, which are connected with a ring, drive the melt. In this section, the set-up will be discussed in more detail, see further Swartjes [137].

\section{Design aspects}

The choice for the depth-to-height ratio, the absolute depth and the length of the channels, will be elucidated. 
In WAXS experiments, information can be obtained for scattering angles (20) lower than 22 degrees for a wavelength of 0.154 [nm] $\left(2 \theta_{\mathrm{Cu}}\right.$; see Table 2.6). The wavelength in synchrotron radiation facilities can be varied, but a wavelength of about 0.7 [ $\mathrm{i}$ ] was used to have an optimum transmission of the X-rays through the diamond windows used in the flow cell. For this wavelength, the maximum scattering angle is about 10 degrees (using Bragg's Law, Equation (2.7)). This gives a limitation for the maximum depth-to-height ratio of the channels of the cross-slot (Figure 6.1(a)), since the relation between this ratio and the maximum scattering angle for a measurement in the stagnation point, that is not influenced by the absorption of the metal corners, is given by

$$
\frac{D}{H}=\frac{1}{\sqrt{2} \tan \alpha}
$$

Choosing a maximum scattering angle of 15 degrees, gives a depth-to-height ratio of 2.64 [-]. For ease of interpretation of the flow, it should be preferable to use depth-toheight ratios larger than eight, to create a two dimensional flow (see Section 2.1.3). Due to the limiting value of 2.64 [-], three-dimensional effects have to be allowed. In order to measure also away from the center of the cell, the depth-to-height ratio is chosen equal to $1.0[-]$.

A sample thickness of 1.0 to 3.0 [mm] was considered to have an optimum transmittance through the sample. Consequently, the depth of the channels was chosen equal to $2[\mathrm{~mm}]$. Using this depth and a maximum scattering angle of 15 degrees, the WAXS measurements have to be performed in a region of 0.54 [mm] from the metal corners. This region is shown in Figure 6.1(b).

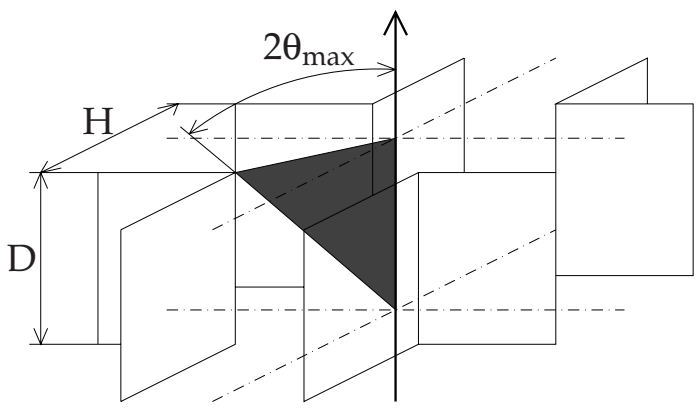

(a)

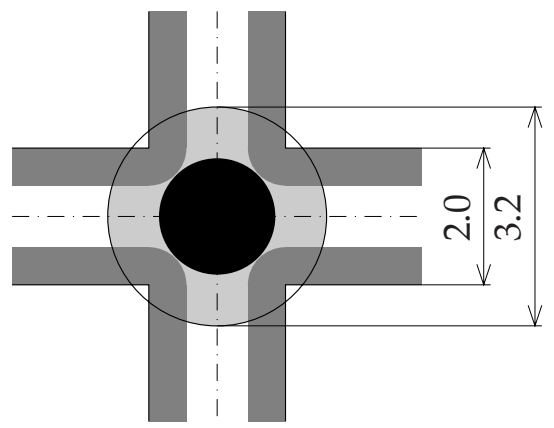

(b)

Figure 6.1: (a) Relation between the depth-to-height ratio and the maximum scattering angle $2 \theta_{\max }$. (b) Measurement region (black) for the WAXS experiments having a diameter of $1.67[\mathrm{~mm}]$. Dark and light grey regions: influence of walls and window holder, respectively (aperture diamond window $=3.2[\mathrm{~mm}]$ ).

The length of the inflow channels has to be sufficiently large to relax the stresses in the melt induced by flow from the reservoir into the inflow channels. The deformation history should be determined mainly by flow close to the stagnation point. A channel length of $46.3[\mathrm{~mm}]$ was calculated using a relaxation time of 10 [s] and a mean velocity of $4.63 \cdot 10^{-3}[\mathrm{~m} / \mathrm{s}]$ (Table 5.1; corresponding to a full stroke of the ring in 40 [s]). 


\section{Final design}

The total set-up is shown schematically in Figure 6.2, and a cross-section of the cell is shown in Figure 6.3. The outer diameter of the flow cell is 130 [mm]. It consists of four cylindrical plates. The dimensions of the inflow and outflow channels are $45 \times 2 \times 2$ $\left[\mathrm{mm}^{2}\right]$ (length $\times$ height $\times$ depth), and of the reservoirs are $5 \times 2\left[\mathrm{~mm}^{2}\right]$ (height $\times$ depth). The cell is fully insulated and fixed to a frame. Insulation reduces heat losses and thermal gradients inside the flow cell.

The flow cell was mounted symmetrically, with respect to the center of the cell, using two bolts to a frame, with insulation material in between, in order to eliminate heating of the frame and a resulting vertical movement. The frame was mounted on a vertical (Newport; M-MTM200PP.1) and a horizontal translation stage (Newport; UZM160PP.05), respectively, to control the position of the cell. Details on these translation stages can be found in Appendix A.

Early test experiments showed the occurrence of shrinkage holes near the stagnation point during crystallization. Two cavities enter the center part of the cell, while material is sucked into the reservoirs. If the ring was moved a full stroke, these cavities were formed by the outflow channels, because at this side most of the sample volume is situated. To correct for this shrinkage and possible leakage, two extra reservoirs were added and connected with the original reservoirs. In this extra reservoir, a sample volume of $\varnothing 8.2 \times 10\left[\mathrm{~mm}^{3}\right]$ was pressurized using a metal spring. (These extra reservoirs are not shown in Figure 6.3(a)).

Flow was created by moving the ring with a DC-motor (Maxon motor, 2260 (diameter $60[\mathrm{~mm}]), 80$ [W] (F program)). Planetary gearing with a ratio of 236:1 was used to decrease the outgoing velocity and increase the maximum allowed torque. The ring was connected with the motor at two sides with steel cables, to load the cell symmetrically in order to avoid a horizontal displacement of the cell. The movement of the ring was measured with a linear displacement transducer (Lucas Schaevitz), connected with one of the driving cables.

Three oil baths (LAUDA K 6 KP and UB 20) were used to impose a desired thermal history. The first oil bath was used to define the annealing temperature $T_{a}$ and flow temperature $\mathrm{T}_{\mathrm{f}}$. The second oil bath was used to cool the cell and was therefore kept at a low temperature $\left(30\left[{ }^{\circ} \mathrm{C}\right]\right)$. The largest oil bath (LAUDA UB 20) was used to define the crystallization temperature $T_{c}$. Due to its higher oil-contents, this bath is less disturbed by changes in the oil temperature. There was no control loop between the oil baths and the flow cell.

The baths were connected with a valve system to the flow cell. This system consists of three mechanical valves (Klinger), which could be opened and closed with pneumatic controlled rotators (Bar $\mathrm{GmbH}$ ). The pneumatic valves (Airtec) were remote controlled. The valve system was designed such that only one oil bath could be connected with the flow cell.

The flow cell was heated by two spiral oil channels in the two inner plates, which are next to the sample. Each channel has its own inflow and outflow connection. For the ease of disconnecting the cell from the thermal system and opening the cell, quick couplings (TEMA) were used. Temperature was measured close to the center and close to the upper reservoir of the cell. 


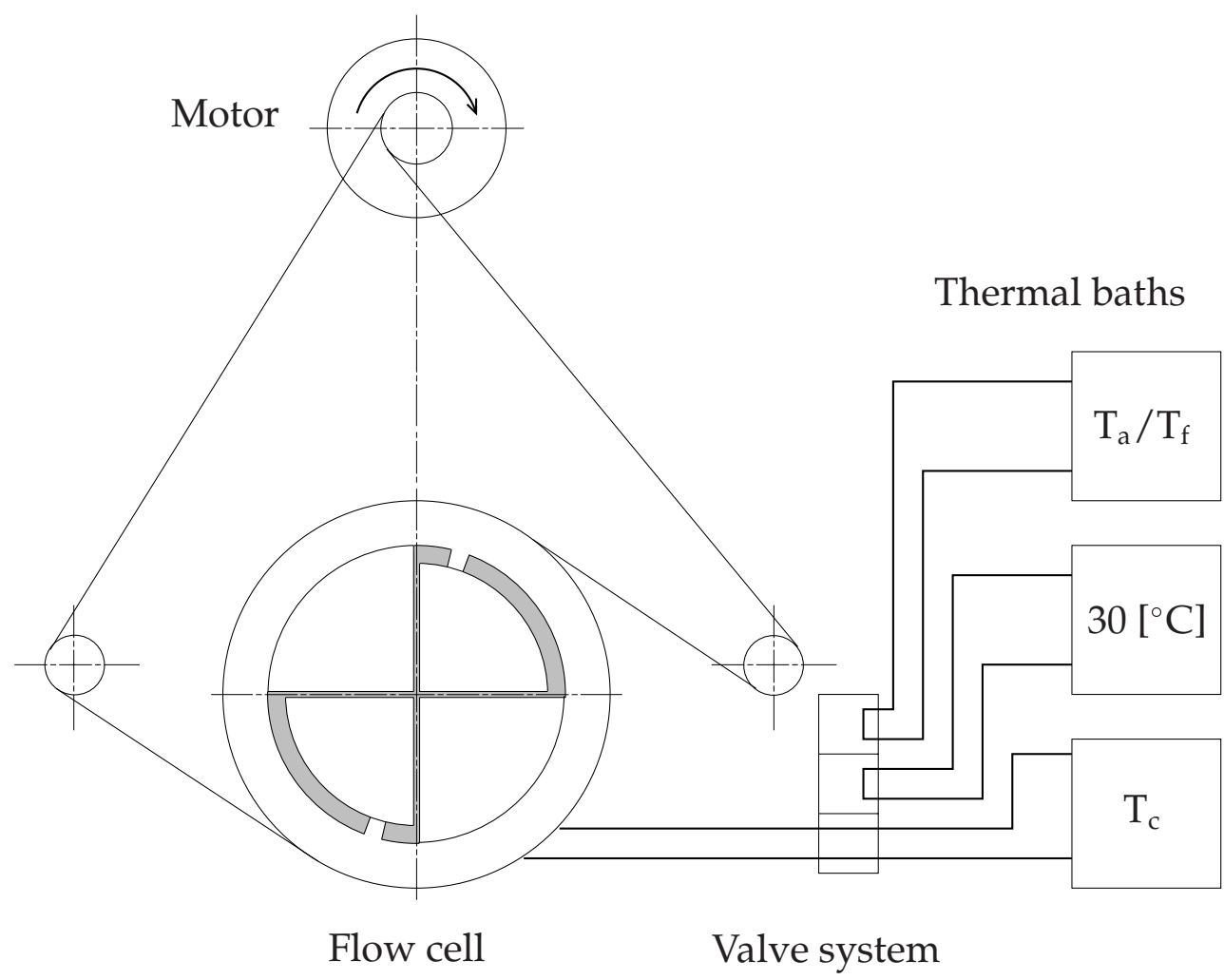

Figure 6.2: Schematic drawing of the cross-slot set-up. Flow is created by moving a ring with two cams. Temperature history is defined by three thermal baths connected with the cell via a valve system.

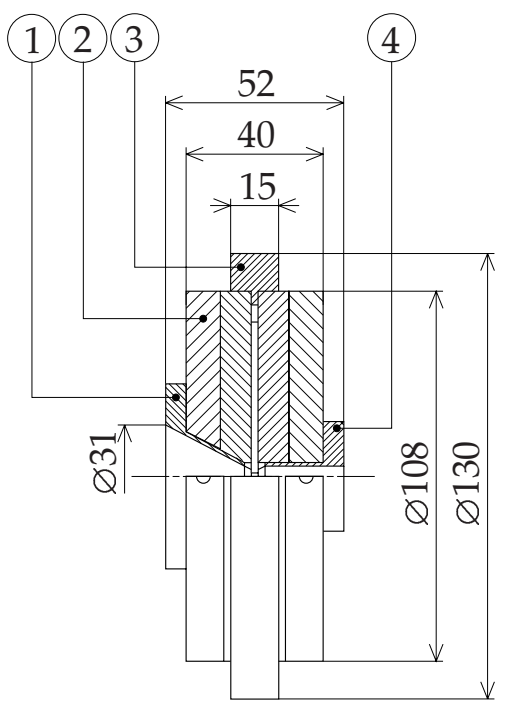

(a)

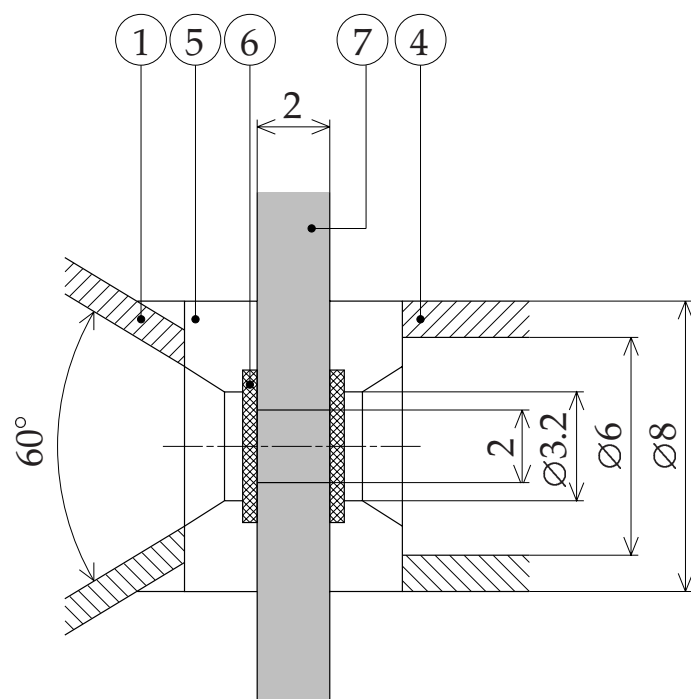

(b)

Figure 6.3: (a) Side view of the flow cell with a cross-section of the upper part. 1. X-ray exit tube; 2. cross-slot consisting of four plates; 3. driving ring with cams; 4. X-ray entrance tube. (b) Detailed drawing of the center of the flow cell. 1. X-ray exit tube; 4. X-ray entrance tube; 5 . window holder; 6 . diamond window; 7 . channel with polymer melt. 
Two different window materials were used: diamond and Schott SF57 glass windows. Diamond windows are used for the X-ray experiments, because the absorption coefficient of this material in the wavelength range $0.7-1.0[\AA]$ is very low. An extra advantage of diamond is the large thermal conductivity, giving almost no thermal gradients in the sample. Unfortunately, the birefringence in the diamond windows was quite high. Birefringent lines indicating stresses in the window were visible between crossed polarizers. Therefore, Schott SF57 glass was used as a window material for the birefringence experiments. The disadvantage of this material is the lower fracture strength and, therefore, the larger window thickness needed. Moreover, thermal conductivity is only slightly larger than for polymer materials, giving larger thermal gradients in the sample. The mechanical, thermal and optical properties of the two window materials are described in Appendix D, including the calculation of the temperature gradients near the windows. Temperature gradients in the polymer channel can be neglected in case of diamond, while they are relatively large (about $0.5\left[{ }^{\circ} \mathrm{C}\right]$ in the depth of the cell) in case of SF57.

The diamond windows (provided by Drukker International, Cuijk, the Netherlands) have a thickness of $0.25[\mathrm{~mm}]$, a diameter of $4.2[\mathrm{~mm}]$, and are glued in a stainless steel frame (clear aperture $3.2[\mathrm{~mm}]$ ) (see Figure 6.3(b)). The diamond windows were polished to an optical surface quality, having the following specifications: parallelism of main sides $\leq 5$ [arcmin], flatness $\leq 650\left[\mathrm{~nm} / \mathrm{mm}^{2}\right]$ and roughness ( $\mathrm{P}-\mathrm{V}$ value) $\leq 20[\mathrm{~nm}]$. The low roughness value was chosen to reduce possible nucleation on the window. However, it is unknown which value is sufficient to neglect this influence.

The low-birefringent windows have a thickness of $1.8[\mathrm{~mm}]$, a diameter of $8[\mathrm{~mm}]$, and are glued to a stainless steel frame (clear aperture $5.0[\mathrm{~mm}]$ ).

\subsection{Birefringence}

Field-wise birefringence experiments were performed to check experimental conditions and to find the useful experimental time range. Only qualitative results for the comparatively high molecular weight material (DSM13E10, $\mathrm{M}_{\mathrm{w}}=500[\mathrm{~kg} / \mathrm{mol}]$ and $\left.\mathrm{M}_{\mathrm{w}} / \mathrm{M}_{\mathrm{n}}=6.0[-]\right)$ are presented.

\subsubsection{Experimental set-up}

A clear overview of polarimeters for linear birefringence measurements is given in Fuller [43, Chapter 8]. Basically, a polarimeter consists of five elements: a light source, a polarization state generator, the sample to be investigated, a polarization state analyzer and a detector.

The optical train for the field-wise birefringence experiments is shown in Figure 6.4. This optical train is fixed, while the flow cell is mounted on a traverse system. A HeNelaser with a wavelength of $632.8[\mathrm{~nm}]$ is used in combination with a beam expander to obtain a parallel light beam with a diameter of about $15[\mathrm{~mm}]$. The first polarizer is used to set the intensity hitting the camera. The polarization state generator consists of a polarizer and a quarter wave plate, which is placed at an orientation angle of 45 degrees relative to the second polarizer. The polarization state analyzer consists also of a 
polarizer and a quarter wave plate, which are placed at an orientation angle of 135 and 90 degrees, respectively, relative to the second polarizer. Orientation of the polymer chains is expected to be in outflow direction. Therefore, the optical train is mounted at 45 degrees with respect to the outflow channel of the flow cell, to obtain the maximum intensity on the outflow centerline (Fuller [43, Section 8.2.2]). The birefringence is visualized with a camera (Panasonic WV-CD130) in combination with a microscope (Zeiss SV-11). Details about the optical components can be found in Appendix A.

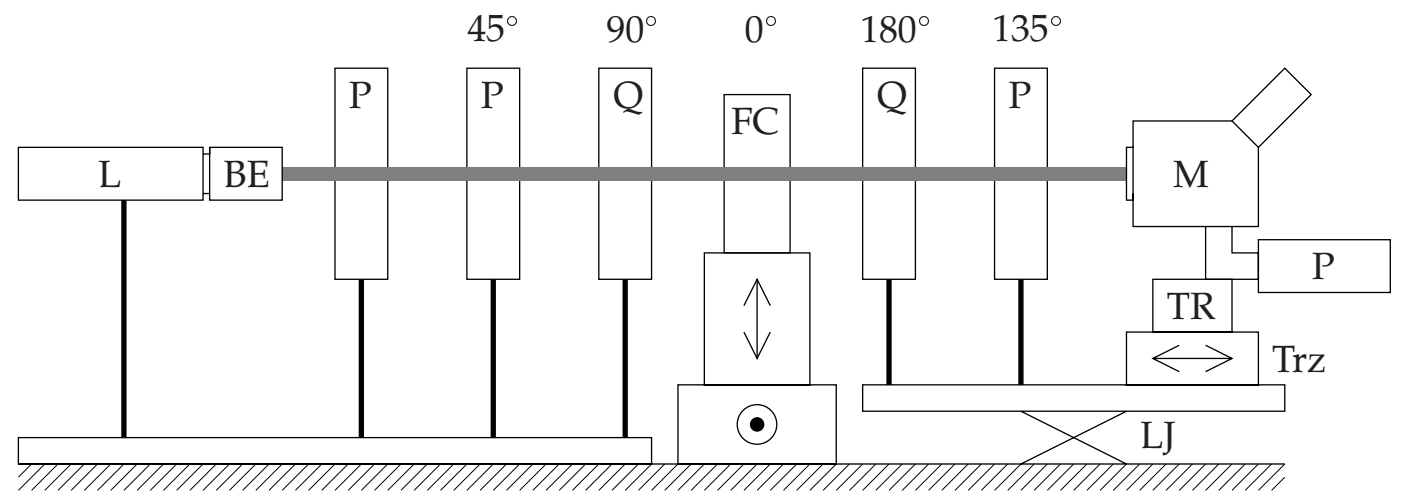

Figure 6.4: Optical train for field-wise birefringence measurements. $\mathrm{L}=$ laser, $\mathrm{BE}=\mathrm{beam}$ expander, $\mathrm{P}=$ polarizer, $\mathrm{Q}=$ quarter wave plate, $\mathrm{FC}=$ flow cell, $\mathrm{M}=$ microscope, $\mathrm{C}=$ camera, $\mathrm{TR}=$ tilt/ rotation stage, $\mathrm{Tr}=$ translation stage in beam direction and $\mathrm{LJ}=$ laboratory jack.

\subsubsection{Experimental results}

Samples were compression moulded at a temperature of $230\left[{ }^{\circ} \mathrm{C}\right]$. With an increment of two minutes the pressing force was increased in seven steps to a maximum of 50 [kN]. A special preform was used to mould the curved parts for the reservoir. The circular parts for the extra reservoirs and the strips for the flow channels were cut out of a moulded plate of $2[\mathrm{~mm}]$ thickness.

Two experiments were performed at a flow and crystallization temperature of 145 and $155\left[{ }^{\circ} \mathrm{C}\right]$, using a low flow rate $\left(\dot{\varepsilon} \approx 0.38\left[\mathrm{~s}^{-1}\right]\right)$. After annealing the sample at $215\left[{ }^{\circ} \mathrm{C}\right]$ for one hour, the sample was slowly cooled, with the third oil bath, to the flow or crystallization temperature. After waiting for about 30 minutes at the flow temperature, a long duration flow was created ( 7 minutes and 22 seconds for the experiment at $145\left[{ }^{\circ} \mathrm{C}\right]$ and 13 minutes for the experiment at $\left.155\left[{ }^{\circ} \mathrm{C}\right]\right)$ to show the flow-induced enhancement of crystallization at rather extreme conditions.

Results of the experiment at $145\left[{ }^{\circ} \mathrm{C}\right]$ are shown in Figure 6.5. One large, broad, flow-induced fringe was found during flow at both ends of the inflow channel. The intensity was high on the outflow centerline, caused by the high orientation of the molecules. A fiber-like structure was found on the outflow centerline (Figure 6.5(a)). Close to this structure other small fringes were found. The high intensity on the centerline did not relax, but remained for at least twenty minutes after the flow was stopped. Typically, the streamlines of the preceding flow can be observed in Figure 6.5(c). This structure is probably (flow-induced) crystallization on and close to the windows. The 
small fringes close to the outflow centerline observed during flow, were also observed after flow (during the first 20 minutes).

The experiment at $155\left[{ }^{\circ} \mathrm{C}\right]$ also showed one large, broad flow-induced fringe at both ends of the inflow channel and the high orientation on the outflow centerline during flow. In this experiment, the birefringence relaxes slowly after cessation of flow (Figure 6.6), indicating that the birefringence was mainly flow-induced (and not due to a crystalline structure). After increasing the contrast, the image 20 [s] after cessation of flow still shows the bright band on the outflow centerline and vaguely the fringe at the end of the inflow channels, after increasing the contrast of the image. Relaxation of the inflow centerline (the black line in Figure 6.6(a)) is shown in Figure 6.6(b). The stagnation point does not remain exactly in the same position. In Figure 6.6(b), the yposition of the stagnation point changes from slightly positive during flow, to a small negative value during relaxation.

The shape of the experimental relaxation curves is compared with the numerically predicted relaxation behavior calculated for DSM13E10. The first normal stress difference for the Leonov and Pompom model are shown in Figure 6.7. The temperature and flow conditions were different, but the comparison is only meant qualitative. The typical narrow bell shaped curves found in the experiments is best predicted by the Pompom model.

The magnitude of the stress at the broad fringe can be estimated. The retardation of the first fringe is equal to $2 \pi$. Using Equation (2.3), gives a birefringence of $3.16 \cdot 10^{-4}$ [-]. The corresponding stress is equal to $3.5 \cdot 10^{5}[\mathrm{~Pa}]$, using a stress optical coefficient of $0.9 \cdot 10^{-9}\left[\mathrm{~Pa}^{-1}\right]$ (Galiatsatos et al. [48]). Due to three-dimensional effects, optical properties are varying along the propagation direction of the light beam. A quantitative comparison can be obtained using the differential propagation matrix theorem (Azzam [5], see also Fuller [43]). Verbeeten et al. [144, 145] showed that the stationary stress levels in the cross-slot flow can be accurately described by the Pompom model. However, a quantitative comparison of the relaxation behavior would be interesting, but is difficult, because the stresses can only be fitted on one fringe. A better comparison is possible by using the point-wise polarization modulation technique of Fuller and Mikkelsen [44] (Rheo Optical Analyzer). Besides obtaining not a discrete, but continuous signal, measurements are much more sensitive using this system. Birefringence as low as $\mathcal{O}\left(10^{-8}\right)$ can be measured instead of $\mathcal{O}\left(10^{-4}\right)$ in the field-wise method (Baaijens [7]). 


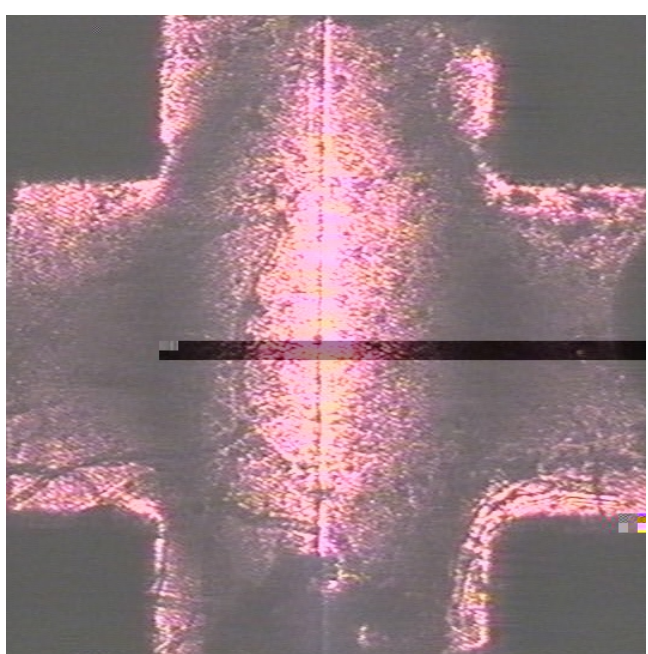

(a)

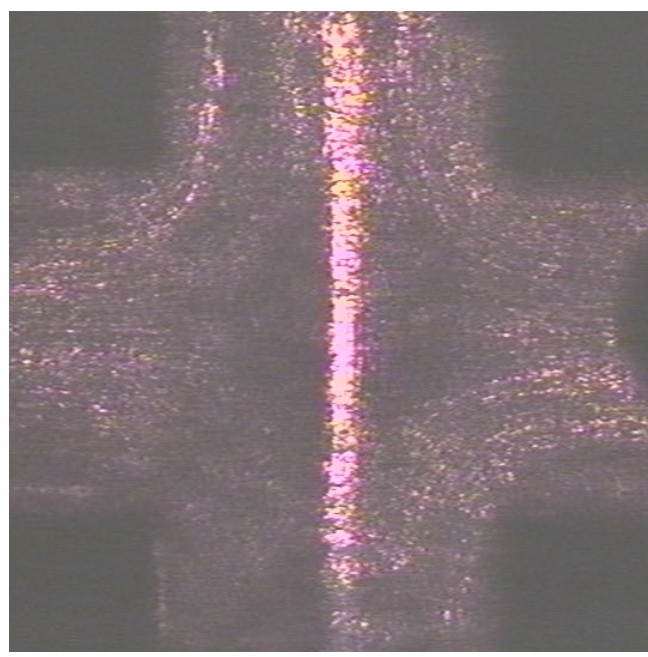

(c)

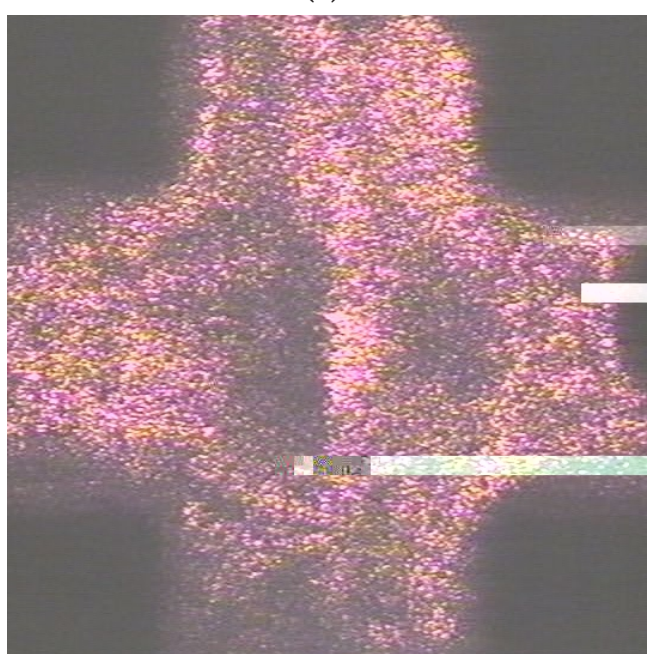

(e)

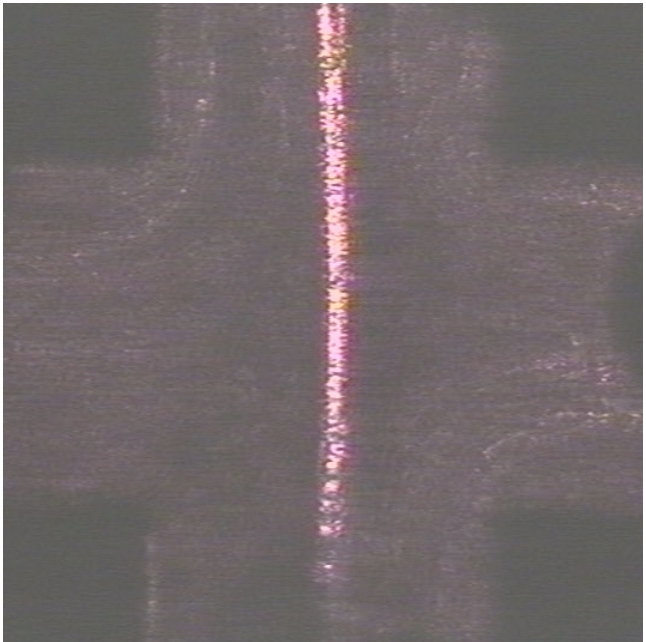

(b)

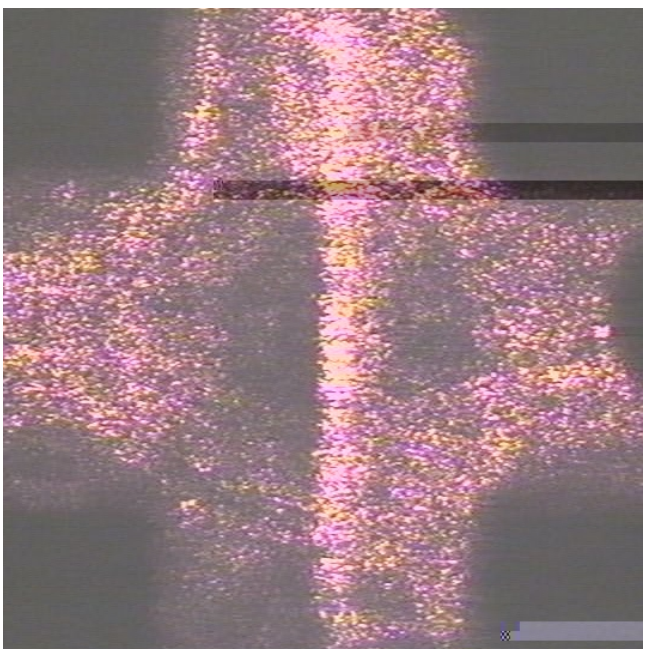

(d)

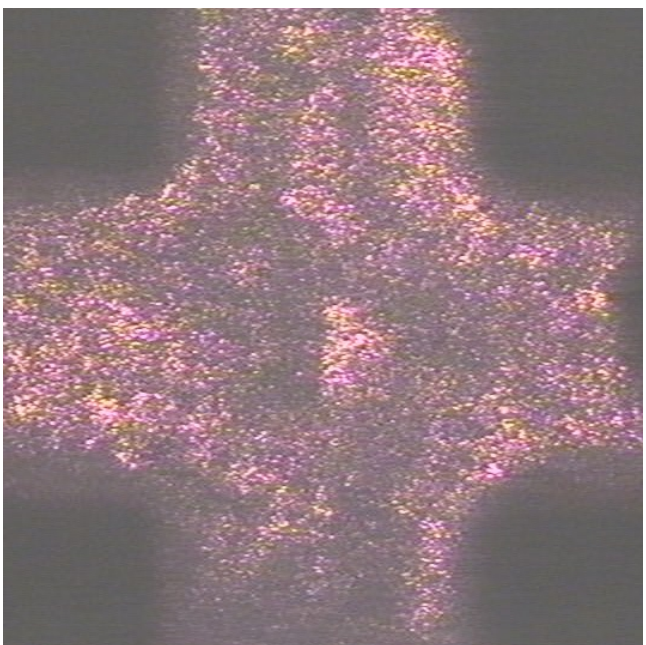

(f)

Figure 6.5: Field-wise birefringence measurement on DSM13E10 in the cross-slot flow cell (a) during flow, three minutes after starting the flow $\left(\mathrm{T}=144.9\left[{ }^{\circ} \mathrm{C}\right]\right.$ and $\left.\dot{\varepsilon} \approx 0.38\left[\mathrm{~s}^{-1}\right]\right)$. Outflow or $\mathrm{x}$-direction is vertical and inflow or y-direction is horizontal. (b) 4 minutes, (c) 10 minutes, (d) 20 minutes, (e) 30 minutes and (f) 40 minutes after cessation of flow $\left(\mathrm{T}=144.9\left[{ }^{\circ} \mathrm{C}\right]\right)$. 


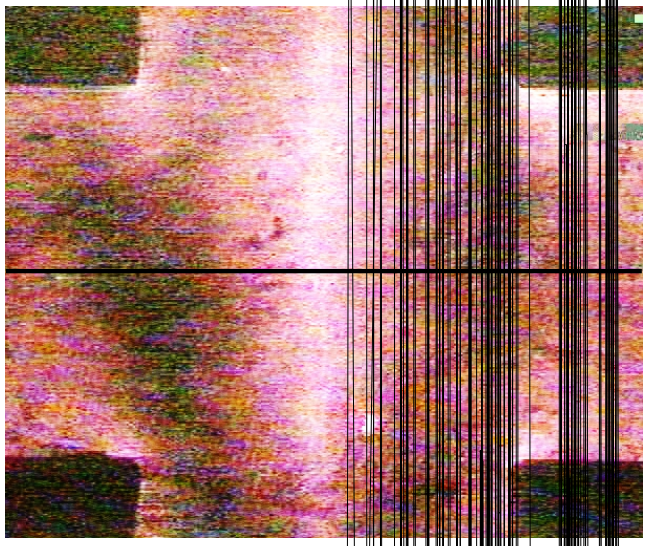

(a)

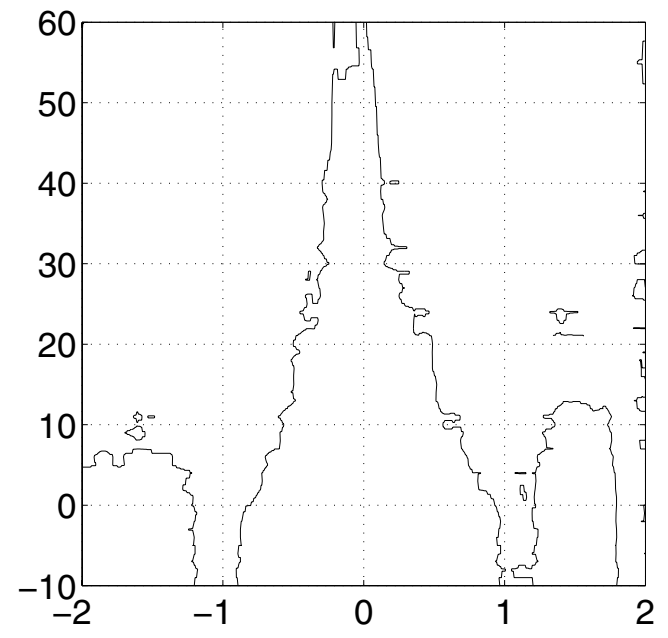




\subsection{WAXS}

WAXS experiments were performed on DSM13E10 at the European Synchrotron Radiation Facility (ESRF) in Grenoble (France) at the micro-focus beam line (ID13), where very small beam sizes down to $2[\mu \mathrm{m}]$ are available, and at the materials science beamline (ID11).

A fotodiode was used to align the flow cell in four steps. First, the cell was roughly aligned on the aperture of the window holder. Since this aperture is not exactly aligned with the cross, three extra steps are necessary to determine the center of the cross. A mesh-scan of 20 horizontal and 20 vertical points was performed around the expected position of one corner (for example between 0.6 and $1.4[\mathrm{~mm}]$ in $\mathrm{x}$ and $\mathrm{y}$ direction). Next, one horizontal and one vertical line-scan of 50 points between -2 and 2 [mm] was performed. Finally, the center of the two obtained intensity curves is defined as the stagnation point. This alignment procedure was repeated after each refill of the flow cell.

Some details of the experimental set-ups of both beam-lines are discussed in the next subsection. All images were corrected for spatial distortion using the 'Fit $2 \mathrm{~d}^{\prime}$ software of the ESRF (Hammersley et al. [56]).

\subsubsection{Experimental set-ups}

\section{ID13}

The experimental set-up at the micro-focus beam-line is shown schematically in Figure 6.8. The beam size depends on the distance of the sample to the collimator that focuses the beam. The collimator-to-sample distance was about 50 [mm], resulting in a beam-size of about $30[\mu \mathrm{m}]$. The lead beam-stop was placed at a distance of about $50[\mathrm{~mm}]$ from the sample. A Photonics Science CCD detector having a measuring area of $92 \times 69\left[\mathrm{~mm}^{2}\right](768 \times 576$ pixels with a horizontal and vertical pixel size of 120 $[\mu \mathrm{m}])$ was used. The exposure time was $8.5[\mathrm{~s}]$, the sample-to-detector distance was $143[\mathrm{~mm}]$, and the wavelength used was $0.785[\AA]$.

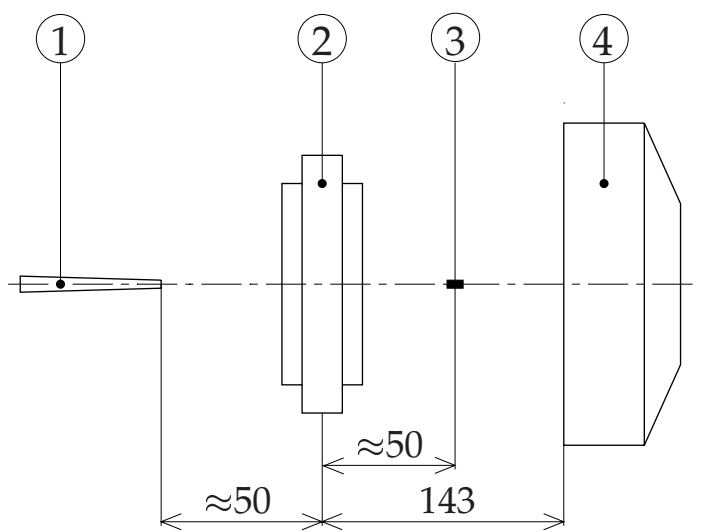

Figure 6.8: Optical arrangement for the $\mathrm{X}$-ray experiments at the micro-focus beam-line (ID13) of the ESRF, Grenoble, France. 1. collimator; 2. flow cell; 3. beam-stop and 4. two dimensional CCD detector 
The set-up, as discussed in Section 6.2, (i.e. flow cell, frame, vertical and horizontal translation stage), was mounted on another horizontal translation stage (MTM200; resolution $1.0[\mu \mathrm{m}])$, which could be moved in the beam direction.

\section{ID11}

A larger beam-size of $0.2 \times 0.2\left[\mathrm{~mm}^{2}\right]$ was used at the materials beam-line. The collimator-to-sample distance and the beam-stop-to-sample distance were about $80[\mathrm{~mm}]$ and 100 [mm], respectively. A Frelon CCD detector having a measuring area of $179 \times 179$ $\left[\mathrm{mm}^{2}\right](1024 \times 1024$ pixels with a horizontal and vertical pixel size of $175[\mu \mathrm{m}])$ and a dynamic range of 14 bit, was used. The exposure time was 5 [s], the sample-to-detector distance was $377[\mathrm{~mm}]$, and the wavelength was $0.718[\AA ̊]$.

\subsubsection{Experimental results}

Measurements on five positions of the outflow centerline were performed at beamline ID11 of the ESRF. A quiescent and a flow experiment were performed sequentially on the same sample. First, the melt was annealed at a temperature of $220\left[{ }^{\circ} \mathrm{C}\right.$ ] for 60 minutes. Next, the sample was cooled (with $15.6\left[{ }^{\circ} \mathrm{C} / \mathrm{min}\right]$ ) to a crystallization temperature of 134.1 [ ${ }^{\circ} \mathrm{C}$ ]. In case of the flow experiment, flow was created at $220\left[{ }^{\circ} \mathrm{C}\right.$ ] for 44 [s], after which it was cooled immediately to $\mathrm{T}_{\mathrm{c}}$. The elongational rate in the stagnation point is estimated to be about $7.0\left[\mathrm{~s}^{-1}\right]$. The undershoot in temperature was equal to 3.2 and $2.5\left[{ }^{\circ} \mathrm{C}\right]$, for the quiescent and flow experiment, respectively.

The two dimensional WAXS patterns were integrated along the Debye ring. Next, the peak area was integrated to obtain a measure for the crystallinity. This value as a function of time for five points on the outflow centerline is shown in Figure 6.9, for the experiments defined above. Differences in crystallization rate are larger in the flow experiment. Moreover, crystallization rates are higher in the flow experiment for all points in comparison to the quiescent experiment. No orientation in the X-ray patterns was found in the the flow experiment. This could be a result of the 'large' beam size used.

It was shown in Chapter 5 that the flow field in the cross-slot results in large stress gradients close to the outflow centerline. Therefore, it is necessary to use a small beamsize to relate the local stresses to the local crystallization behavior. By measuring at several points in the cell, it should be possible to correlate the influence of the difference in deformation history to crystallization for the same thermal history (if there are no gradients), within one experiment. The combination of a high flux and a micro-focus beam in order to measure in-situ structure development locally, is fulfilled at the microfocus beam-line ID13 of the European Synchrotron Radiation Facilities.

Measurements were performed at the micro-focus beam-line to investigate the structure on the inflow and outflow centerline close to the stagnation point. About the same experimental conditions were applied as for the birefringence experiment shown in Figure 6.5.

The sample was annealed at a temperature of $220\left[{ }^{\circ} \mathrm{C}\right]$ for 90 minutes. Next, it was cooled (with $32.7\left[{ }^{\circ} \mathrm{C} / \mathrm{min}\right]$ ) to a flow and crystallization temperature of $148.4\left[{ }^{\circ} \mathrm{C}\right]$. Flow was applied at this temperature to enhance the effects, since relaxation of stresses 
will be limited. Finally, a low flow rate $\left(\dot{\varepsilon} \approx 0.38\left[\mathrm{~s}^{-1}\right]\right)$ was applied for 13 minutes and 39 seconds after waiting about 30 minutes, and crystallization was monitored.

During flow no crystalline structures were found in nine points around the stagnation point $(\mathrm{x}=-0.8: 0.8: 0.8[\mathrm{~mm}]$ and $\mathrm{y}=-0.8: 0.8: 0.8[\mathrm{~mm}])$. Crystalline peaks were observed from about 200 [s] after cessation of flow. The reflections were weak on the inflow centerline and only (040) was oriented parallel to the outflow direction (Figure 6.10(a)). Close to the stagnation point, in the stagnation point and at the outflow centerline, both (110), (040) and (130) were oriented in the outflow direction (Figure $6.10(\mathrm{~b}))$. Notice that the stagnation point is not found at point $(0,0)$. This was also observed in the birefringence experiments.

The integrated WAXS intensity for the inflow and outflow centerline is shown in Figure 6.11. The peak intensities are larger close to the stagnation point on the inflow centerline. On the outflow centerline, it seems that the intensities are more pronounced further from the stagnation point. The size of this oriented structure, with orientation in (110), is $80[\mu \mathrm{m}]$ in inflow (Figure 6.12(a)) and at least 2 [mm] in outflow direction (Figure 6.12(b)). Orientation in (040) was found in all points (Figure 6.13). The same oriented structures were found by others as discussed in Figure 2.5. Orientation in both (110) and (130) corresponds to a highly oriented, non-branched structure.

Recently, other WAXS experiments were performed to investigate the influence of flow above the melting temperature (i.e. at $170\left[{ }^{\circ} \mathrm{C}\right]$ ) on the crystallization behavior around $140\left[{ }^{\circ} \mathrm{C}\right]$. Elongational rates up to $7\left[\mathrm{~s}^{-1}\right]$ were used for DSM13E10 and up to $15\left[\mathrm{~s}^{-1}\right.$ ] for DSM15M10. Due to the high viscosity of DSM13E10, larger flow rates were not possible at a temperature of $170\left[{ }^{\circ} \mathrm{C}\right]$ with the current set-up. In these isothermal experiments, a sudden drop in the intensity was observed for points close to the outflow centerline. Generally, a drop in intensity can be explained by a change in the temperature, a change in the flux of the synchrotron source or a change in the amount of material. The first explanation is not applicable to these isothermal experiments. The flux of the X-ray beam decreases only gradually in time, due to the decrease of the amount of electrons in the storage ring. Therefore, the sudden change in intensity during an isothermal experiment is ascribed to the development of a shrinkage hole. Such hole can have a diameter of about $0.4[\mathrm{~mm}]$ (determined from the decrease in intensity). Simultanously, large orientations were observed, which are ascribed to the local flow caused by the developing shrinkage holes. The integrated intensity in time was checked for all experiments, and only the measurements that show no decrease in intensity were shown above.

More disturbing is that the influence of the stagnation point (before the occurrence of the shrinkage holes) is much less pronounced than expected and anticipated during the design of the cross-slot flow cell. Therefore, three-dimensional effects are investigated in the next section to clarify the influence of the shear gradients in the depth of the flow cell. 


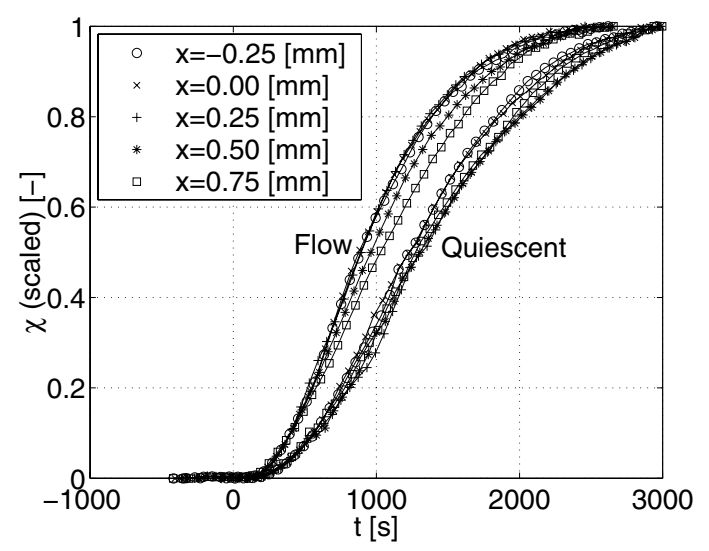

Figure 6.9: Scaled crystallinity against time in five points on the outflow centerline $(y=0[\mathrm{~mm}])$ of the cross-slot at $\mathrm{T}_{\mathrm{C}}=134.1\left[{ }^{\circ} \mathrm{C}\right]$. The elongational rate in the flow experiment was about $7.0\left[\mathrm{~s}^{-1}\right]$. The measurement was performed at the ID11 of the ESRF (Grenoble, France) using a beam-size of $0.2 \times 0.2\left[\mathrm{~mm}^{2}\right]$.

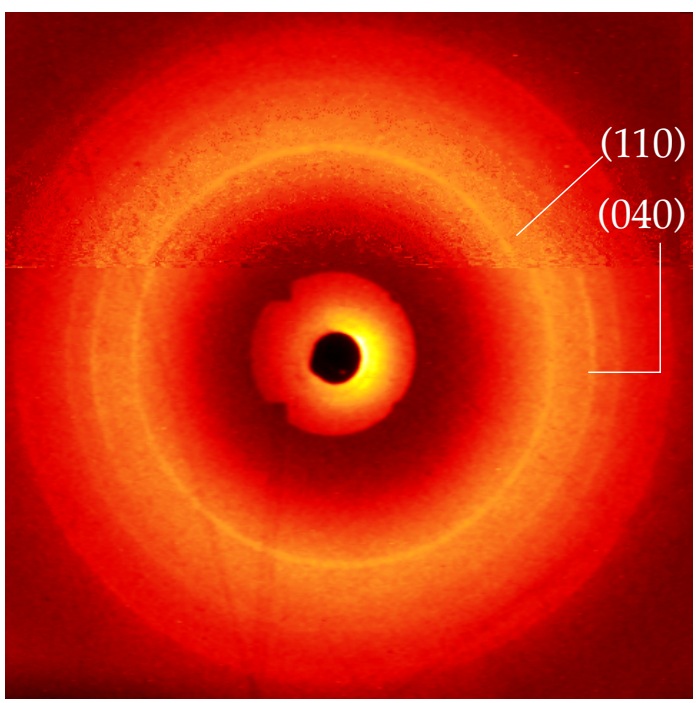

(a)

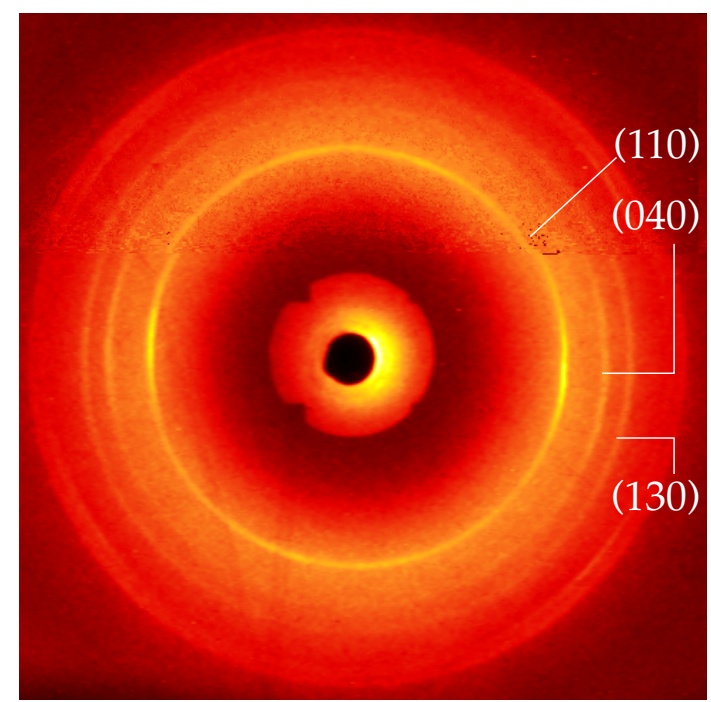

(b)

Figure 6.10: $2 \mathrm{D}$ WAXS patterns around stagnation point for DSM13E10 at $\mathrm{T}=148.4\left[{ }^{\circ} \mathrm{C}\right]$ and after $\dot{\varepsilon} \approx 0.38\left[\mathrm{~s}^{-1}\right]$. (a) Inflow centerline ( $\mathrm{x}=0[\mathrm{~mm}]$ and $\left.\mathrm{y}=-0.16[\mathrm{~mm}]\right), 6173$ [s] after cessation of flow. (b) In stagnation point $(x=0[\mathrm{~mm}]$ and $y=-0.06[\mathrm{~mm}]), 6248[\mathrm{~s}]$ after cessation of flow. 


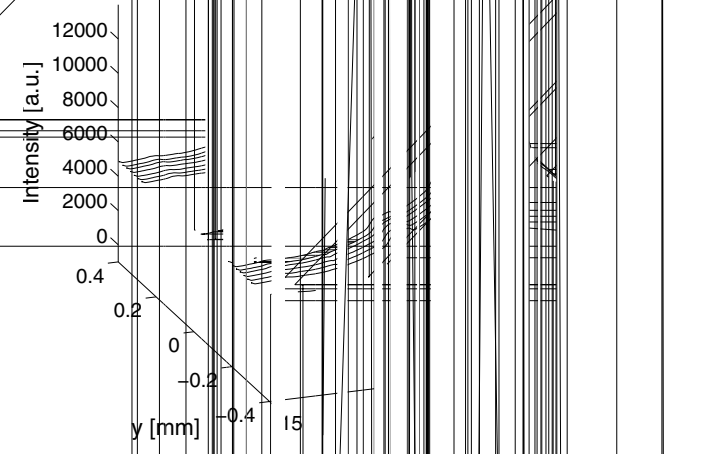




\subsection{Numerical predictions}

The influence of three-dimensional effects is numerically investigated to clarify the less pronounced influence of the stagnation point in the X-ray measurements when flow was applied above the melting temperature. The parameters and fits of Daplen KS10 are used (see Table 4.7).

A flow rate of $1.85 \cdot 10^{-8}\left[\mathrm{~m}^{3} / \mathrm{s}\right.$ ] (in one inflow channel) for 40 [s] was used at a temperature of $200\left[{ }^{\circ} \mathrm{C}\right]$. Next, the sample was cooled using a cooling rate of $30\left[{ }^{\circ} \mathrm{C} / \mathrm{min}\right.$ ] to the isothermal crystallization temperature of $140\left[{ }^{\circ} \mathrm{C}\right]$. Time $\mathrm{t}=0[\mathrm{~s}]$ is defined as the time reaching $\mathrm{T}_{\mathrm{c}}$. The space filling during crystallization was calculated in ten planes in depth $(\mathrm{z}=0.05: 0.1: 0.95[\mathrm{~mm}])$ for 150 points. Some extra points were used close to the outflow centerline.

As a comparison, the predicted space filling in plane $\mathrm{z}=0.05[\mathrm{~mm}]$ and $\mathrm{z}=0.95[\mathrm{~mm}]$ are shown in Figure 6.14. Of course, the prediction of plane $\mathrm{z}=0.05[\mathrm{~mm}]$ looks very similar to Figure 5.8. Large values are found close to the outflow stagnation line and the corner. The influence of the shear rate in the $\mathrm{z}$-direction is clearly shown in Figure $6.14(\mathrm{~b})$. The average space filling is raised to a value of about 0.40 [-]. Interesting is the increasing value close to the outflow centerline with increasing $\mathrm{x}$-coordinate. The low value for the space filling at the corner is caused by the low shear rates in this region.

The averaged space filling (mean value of the space fillings in the ten planes) is shown in Figure 6.15(a). The behavior looks similar to Figure 6.14, but the flowinduced parts are less pronounced. Moreover, an increasing space filling with increasing position on the outflow centerline is still observed. It is shown here that shear effects in the neutral or viewing direction enhance this effect.

The dependence of space filling on the depth of the cell in the four outer points of the computation region is shown in Figure 6.15(b). The value close to the stagnation point decreases with depth and increases slightly close to the wall. The point close to the corner decreases continuously with depth, while the point on the inflow centerline increases with depth. This point shows most clearly the influence of the shear rate in neutral direction, because its value is zero in absence of this shear rate (in plane $\mathrm{z}=0[\mathrm{~mm}])$. The largest changes are found in the point on the outflow centerline. Initially, it is very close to the value in the stagnation point and decreases with depth, but it increases rapidly close to the wall.

It is shown that for the experimental settings and the chosen depth-to-height ratio of the flow channels, the three-dimensional effects can be considerable. Next, the influence of the flow time, the flow rate, the flow temperature, the depth-to-height ratio and the rounding of corner are discussed.

\section{Flow time}

An infinite residence time is only obtained (theoretically) for a particle in the stagnation point. In practice, this is not interesting because this particle has no dimensions. The residence time in other points is finite and depends on the position and the flow rate.

The residence time of the particles in the finite element part of the cell is only in the order of a few seconds for this large flow rate of $1.85 \cdot 10^{-8}\left[\mathrm{~m}^{3} / \mathrm{s}\right]$. For example, it 


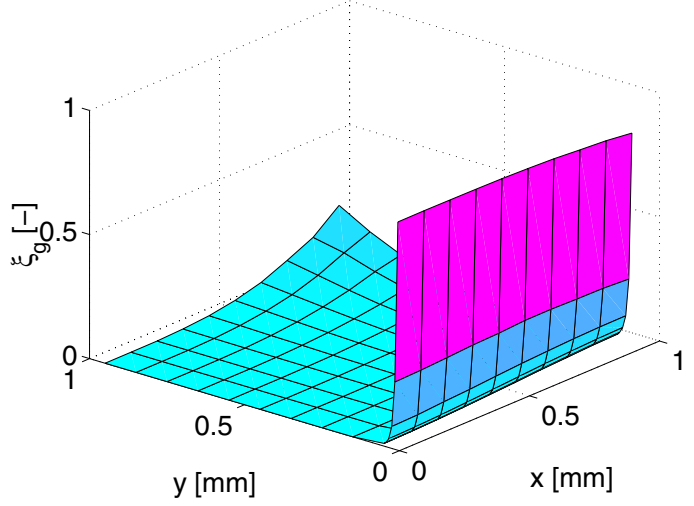

(a)

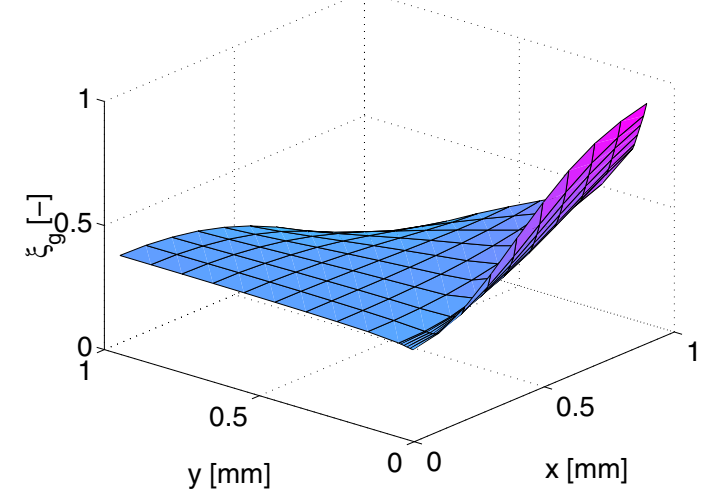

(b)

Figure 6.14: Prediction of the space filling in the cross-slot flow cell for Daplen KS10 after applying a flow rate of $1.85 \cdot 10^{-8}\left[\mathrm{~m}^{3} / \mathrm{s}\right]$ for $40[\mathrm{~s}]$ at $200\left[{ }^{\circ} \mathrm{C}\right]$, subsequent cooling to $140\left[{ }^{\circ} \mathrm{C}\right]$, and waiting $1200[\mathrm{~s}]$, using the $\mathrm{S}_{\mathrm{J} 2}$ model. (a) plane $\mathrm{z}=0.05[\mathrm{~mm}]$ and (b) plane $\mathrm{z}=0.95[\mathrm{~mm}]$.

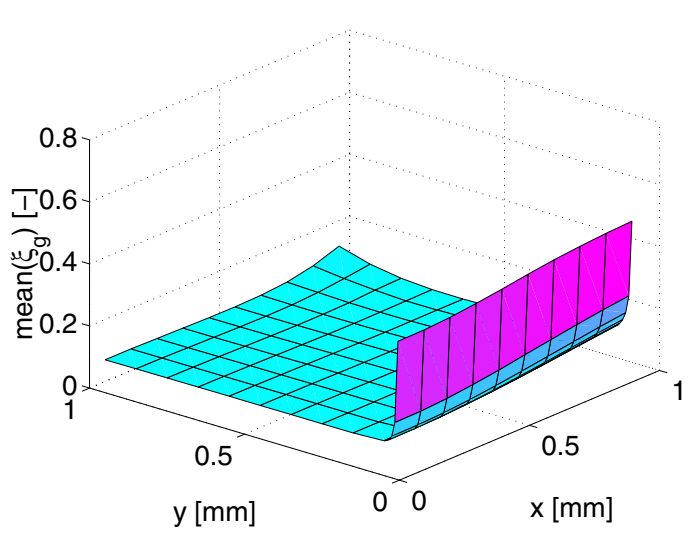

(a)

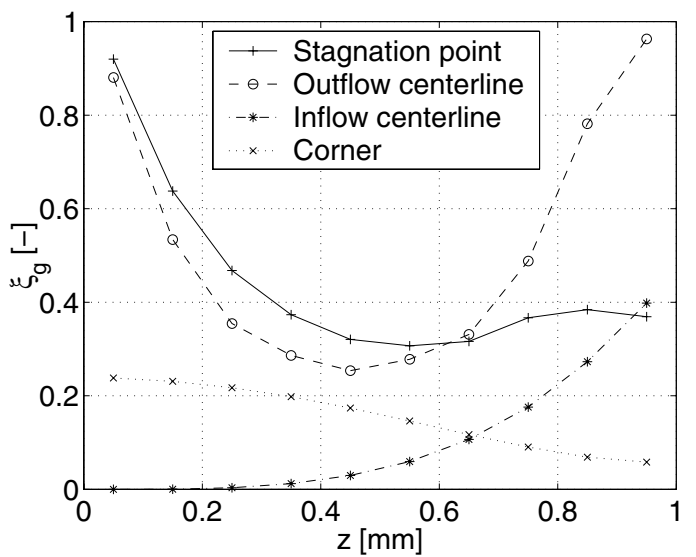

(b)

Figure 6.15: (a) Prediction of the mean space filling in the depth of the cross-slot flow cell for Daplen KS10, using the $\mathrm{S}_{22}$ model (conditions as in Figure 6.14). (b) Prediction of the space filling at four points in the cross-slot flow cell for Daplen KS10 (conditions as in Figure 6.14). The positions $(\mathrm{x}, \mathrm{y})$ in $[\mathrm{mm}]$ are: stagnation point $\left(1 \cdot 10^{-6}\right.$, $\left.5 \cdot 10^{-3}\right)$, point on the outflow centerline $\left(0.9,5 \cdot 10^{-3}\right)$ and on the inflow centerline $\left(1 \cdot 10^{-6}, 0.95\right)$, and a point near the corner $(0.9,0.95)$. 
is about 0.7 [s] in plane $\mathrm{z}=0.05[\mathrm{~mm}]$ for a point close to the stagnation point $\left(1 \cdot 10^{-6}\right.$, $\left.5 \cdot 10^{-3}\right)$ and on the outflow centerline $\left(0.9,5 \cdot 10^{-3}\right)$, and in plane $\mathrm{z}=0.95[\mathrm{~mm}]$ it is $7.4[\mathrm{~s}]$ for a point close to the stagnation point $\left(1 \cdot 10^{-6}, 5 \cdot 10^{-3}\right)$ and $5.5[\mathrm{~s}]$ for a point on the outflow centerline $\left(0.9,5 \cdot 10^{-3}\right)$. This means that it is not necessary to use the full stroke of the ring, which in this case corresponds to an experimental time of 40 [s], to obtain the maximum orientation around the stagnation point. A shorter experimental time would give about the same results for points close to the outflow stagnation line and less influence of shear rates as shown in Figure 6.16. The space filling values near the corner in plane $\mathrm{z}=0.05[\mathrm{~mm}]$ and the mean value in plane $\mathrm{z}=0.95[\mathrm{~mm}]$ are lowered, but the values close to the outflow stagnation line are about the same.

\section{Flow rate}

Lowering the flow rate, lowers the total strain for particles that suffered shear gradients. However, the total strain for particles close to the outflow stagnation line is independent of the flow rate, because particles travel with a lower velocity, but also for a longer time (see also Schoonen [128]).

The flow rate is lowered with a factor 10 to a value of $1.85 \cdot 10^{-9}\left[\mathrm{~m}^{3} / \mathrm{s}\right]$. The results for two planes $(\mathrm{z}=0.05[\mathrm{~mm}]$ and $\mathrm{z}=0.95[\mathrm{~mm}])$ are shown in Figure 6.17. The influence of the shear rates is smaller with respect to the maximum found close to the outflow stagnation line.

The dependence of the rate of crystallization on the shear rate applied was observed in Chapter 4, by Vleeshouwers and Meijer [147] and predicted by Zuidema et al. [157].

\section{Flow temperature}

Applying the same flow rate at a lower temperature will cause apparent larger shear rates. (This can be argued by shifting a shear rate at a certain temperature to a (higher) reference temperature by multiplying it with a value larger than 1 (Table 4.2).) Therefore, the flow will be more plug-like and three-dimensional effects will be smaller.

\section{Depth-to-height ratio}

Increasing the depth-to-height ratio of the channels within the cell will reduce the three-dimensional effects. A calculation was performed for a depth-to-height ratio of $2[-]$, using the same amount of spectral elements as in the other calculations (Figure 5.2(b)), but with some refinement to the outer wall. The results for two planes $(\mathrm{z}=0.05 \times \mathrm{D}[\mathrm{mm}]$ and $\mathrm{z}=0.95 \times \mathrm{D}[\mathrm{mm}]$, with $\mathrm{D}=2[\mathrm{~mm}])$ are shown in Figure 6.18. The influence of $\dot{\gamma}_{12}$, close to the corner in plane $\mathrm{z}=0.05$ [mm] is not changed, but the influence of $\dot{\gamma}_{13}$ in the plane $\mathrm{z}=0.95 \times \mathrm{D}[\mathrm{mm}]$ is lowered from 0.40 [-] to $0.16[-]$.

\section{Rounding of corner}

Instead of the 90 degrees corners in the cross-slot, a radius could have been introduced. The influence of a radius on the three-dimensional effects was shown in Swartjes [136, Appendix G]. A radius decreases the actual channel depth-to-height ratio 


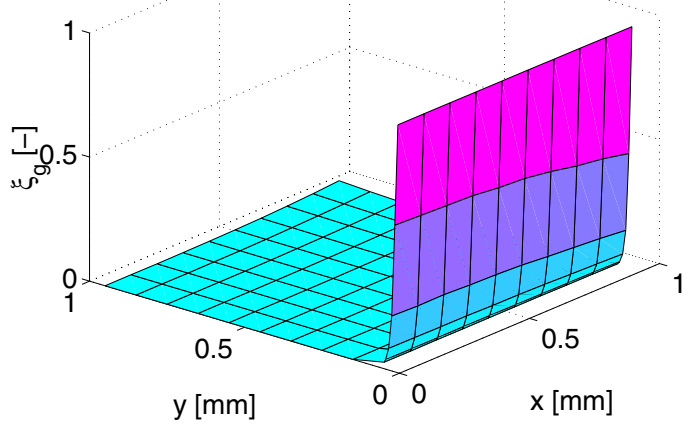

(a)

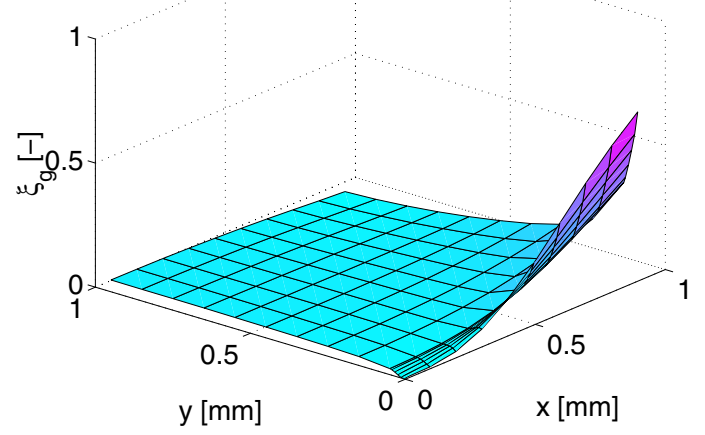

(b)

Figure 6.16: Prediction of the space filling in the cross-slot flow cell (D/H=1 [-]) for Daplen KS10 after applying a flow rate of $1.85 \cdot 10^{-8}\left[\mathrm{~m}^{3} / \mathrm{s}\right]$ for $5[\mathrm{~s}]$ at $200\left[{ }^{\circ} \mathrm{C}\right]$, subsequent cooling to $140\left[{ }^{\circ} \mathrm{C}\right]$, and waiting 1200 [s], using the $\mathrm{S}_{\mathrm{J} 2}$ model. (a) plane $\mathrm{z}=0.05[\mathrm{~mm}]$ and (b) plane $\mathrm{z}=0.95[\mathrm{~mm}]$.

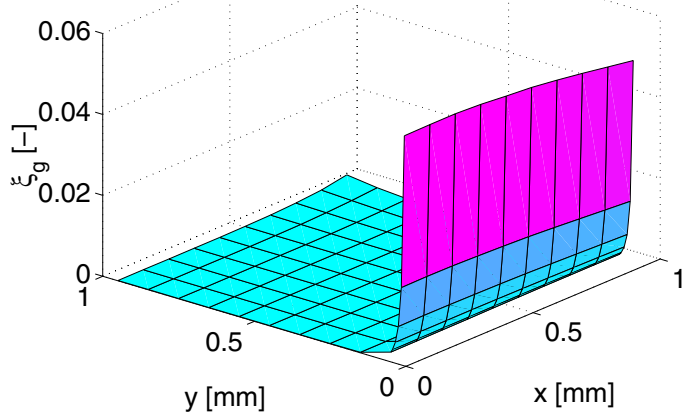

(a)

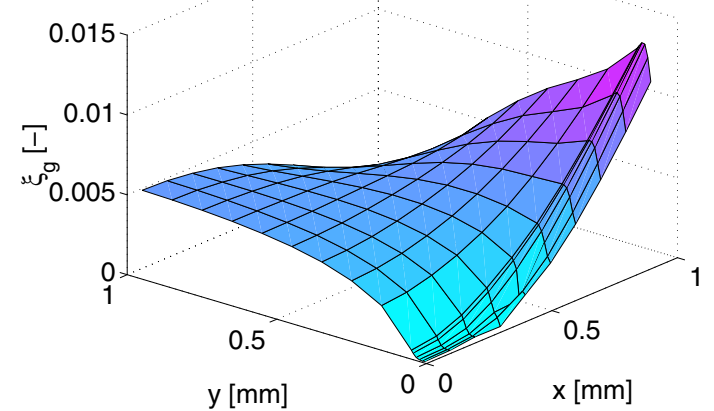

(b)

Figure 6.17: Prediction of the space filling in the cross-slot flow cell (D/H=1 [-]) for Daplen KS10 after applying a flow rate of $1.85 \cdot 10^{-9}\left[\mathrm{~m}^{3} / \mathrm{s}\right]$ for $40[\mathrm{~s}]$ at $200\left[{ }^{\circ} \mathrm{C}\right]$, subsequent cooling to $140\left[{ }^{\circ} \mathrm{C}\right]$, and waiting $1200[\mathrm{~s}]$, using the $\mathrm{S}_{22}$ model. (a) plane $\mathrm{z}=0.05[\mathrm{~mm}]$ and (b) plane $\mathrm{z}=0.95[\mathrm{~mm}]$.

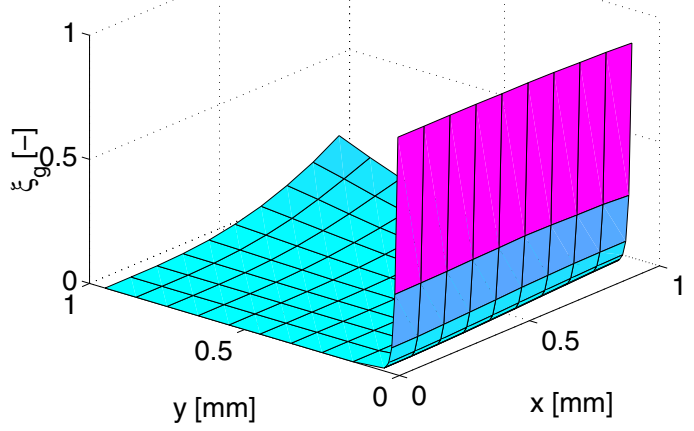

(a)

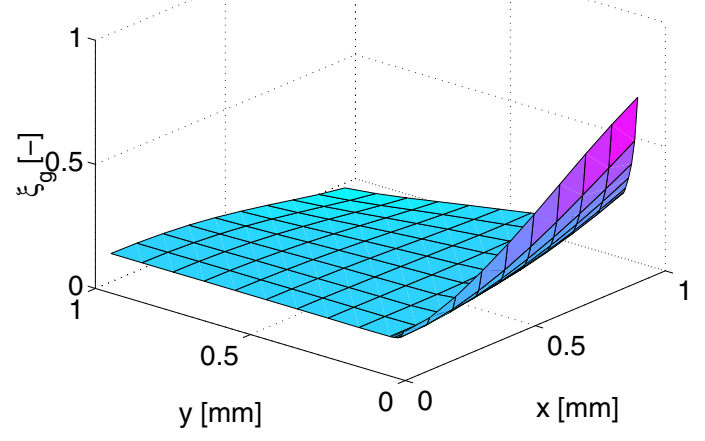

(b)

Figure 6.18: Prediction of the space filling in the cross-slot flow cell (D/H=2 [-]) for Daplen KS10 after applying a flow rate of $1.85 \cdot 10^{-8}\left[\mathrm{~m}^{3} / \mathrm{s}\right]$ for $40[\mathrm{~s}]$ at $200\left[{ }^{\circ} \mathrm{C}\right]$, subsequent cooling to $140\left[{ }^{\circ} \mathrm{C}\right]$, and waiting 1200 [s], using the $S_{22}$ model. (a) plane $\mathrm{z}=0.05 \times \mathrm{D}[\mathrm{mm}]$ and $\mathbf{( b )}$ plane $\mathrm{z}=0.95 \times \mathrm{D}[\mathrm{mm}]$. 
and therefore, increases three-dimensional effects. It was shown that for a radius $\mathrm{R}<0.5 \times \mathrm{H}$ ( $\mathrm{H}=$ channel height and $\mathrm{R}=$ radius) three-dimensional effects are small, while for $\mathrm{R}>0.5 \times \mathrm{H}$ these effects are more pronounced. In the second case, two maxima in the elongation rate on the outflow centerline were observed, namely close to the stagnation point and a second one, between $0.5 \times \mathrm{H}$ and $0.5 \times \mathrm{H}+\mathrm{R}$, caused by the contraction introduced by the curved part of the outflow channel.

\section{Conclusions}

The influence of the shear rate $\dot{\gamma}_{12}$ can be found in the point close to the corner in plane $0.05 \times \mathrm{D}$, while the influence of $\dot{\gamma}_{13}$ can be seen on the inflow centerline in plane $0.95 \times \mathrm{D}$. The results for the calculations shown in Figures 6.16-6.18. are summarized in Table 6.1. The space filling values close to the corner in plane $\mathrm{z}=0.05 \times \mathrm{D}\left(\xi_{\mathrm{g} 12}\right)$ and on the inflow centerline in plane $\mathrm{z}=0.95 \times \mathrm{D}\left(\xi_{\mathrm{g} 13}\right)$ are given, and normalized with the space filling value close to the stagnation point $\left(1 \cdot 10^{-6}, 5 \cdot 10^{-3}\right)$ in plane $\mathrm{z}=0.05 \times \mathrm{D}$. The first value describes the influence of $\dot{\gamma}_{12}$, while the second describes the influence of $\dot{\gamma}_{13}$.

A lower value of the flow rate or a larger depth-to-height ratio reduces the influence of $\dot{\gamma}_{13}$ with respect to $\dot{\gamma}_{12}$. A large influence is seen in reducing the flow time.

\begin{tabular}{|l|r|r||r|r|}
\hline $\mathrm{Q}\left[\mathrm{m}^{3} / \mathrm{s}\right]$ & \multicolumn{1}{|c|}{$\mathrm{t}_{\mathrm{f}}[\mathrm{s}]$} & $\mathrm{d} / \mathrm{h}[-]$ & $\xi_{\mathrm{g} 12}[\%]$ & $\xi_{\mathrm{g} 13}[\%]$ \\
\hline \hline $1.85 \cdot 10^{-8}$ & 40 & 1 & 20.8 & 36.0 \\
\hline $1.85 \cdot 10^{-8}$ & 5 & 1 & 1.8 & 3.3 \\
\hline $1.85 \cdot 10^{-9}$ & 40 & 1 & 14.7 & 9.7 \\
\hline $1.85 \cdot 10^{-8}$ & 40 & 2 & 17.5 & 12.8 \\
\hline
\end{tabular}

Table 6.1: Influence of the variation of the flow rate $Q$, flow time $t_{f}$ and the depth-to-height ratio on the relative space filling. $\xi_{\mathrm{g} 12}$ gives the influence of $\dot{\gamma}_{12}$ and is defined as $\xi_{\mathrm{g}}(0.9,0.95,0.05 \times \mathrm{D}) / \xi_{\mathrm{g}}\left(1 \cdot 10^{-6}, 5 \cdot 10^{-3}, 0.05 \times \mathrm{D}\right) . \xi_{\mathrm{g} 12}$ gives the influence of $\dot{\gamma}_{13}$ and is defined as $\xi_{\mathrm{g}}\left(1 \cdot 10^{-6}, 0.95,0.95 \times \mathrm{D}\right) / \xi_{\mathrm{g}}\left(1 \cdot 10^{-6}, 5 \cdot 10^{-3}, 0.05 \times \mathrm{D}\right)$.

In conclusion, the influence of the stagnation point is more pronounced for shorter flow times (but equal residence time) and larger depth-to-height ratios. The influence of flow rate on crystallization is the unknown relation and, therefore, the flow rate should be a variable.

\subsection{Discussion}

Birefringence and Wide Angle X-ray experiments were performed in a cross-slot flow cell. The birefringence experiments showed the occurrence of 'streamlines' caused by the presence of the front and back wall (the optical windows). This phenomenon can be explained by the influence of shear gradients close to the windows. Moreover, the Schott SF57 glass windows used in these experiments introduce a thermal gradient in the depth of the cell, enhancing crystallization close to the windows. The WAXS 
experiment showed a highly oriented structure close to the outflow stagnation line, having orientation in the (110), (040) and (130) reflections.

Originally, the flow cell was designed to create flow (up to elongational rates of about $10\left[\mathrm{~s}^{-1}\right]$ ) at a temperature above the melting temperature, and investigate crystallization at a low temperature. Drawback of such experiments is that relaxation of the stresses occurs, depending on the cooling rate of the set-up.

WAXS experiments showed that large flow rates also create a large influence of the shear gradients in the height and depth of the flow channel. The effects of flow on crystallization close to the outflow centerline was not as pronounced as expected, when compared with crystallization in the channels and reservoirs. Moreover, this effect is enhanced by a thermal gradient in the flow cell. The temperature close to the cross is about $0.4\left[{ }^{\circ} \mathrm{C}\right]$ higher than close to the reservoir.

The influence of the shear gradients can be reduced by using a shorter flow time and/or using a cell with a larger depth-to-height ratio. However, the experimental set-up should be able to allow for experiments with large elongational rates also at temperatures below the melting temperature, to investigate the influence of relaxation. This is not possible with the current set-up, because the driving wires applied now cannot transfer the necessary large forces. Moreover, larger cooling rates should be used to enhance the flow-induced crystallization effect.

Larger cooling rates can be obtained if the heat capacity of the cell and the total set-up is reduced. During cooling not only the cell has to be cooled, but also a part of the valve system and the tubes. The total length of the tubes is about $2[\mathrm{~m}]$. A second drawback of the current heating system is that the temperature in the cell is not controlled, but steered. The reproducibility of the isothermal crystallization temperature was not good enough, and, therefore, the temperature of the crystallization bath was checked in each experiment, before melting the sample.

A solution would be to heat the cell electrically and to cool the cell with liquid nitrogen to obtain larger cooling rates. The electrical heating gives a better control of the thermal history. It should be able to define the heating and cooling rate using a control loop, which also gives a better reproducible isothermal crystallization temperature. If the electrical heating is mounted at the outside, the temperature will be lower close to the cross, increasing the crystallization behavior close to the stagnation point. In this case, the thermal gradient within the cell is used to enhance flow-induced crystallization in the center of the cross. 


\section{Chapter 7}

\section{Conclusions and recommendations}

A cross-slot flow cell was used to investigate stress-induced crystallization in a complex flow with a strong elongational component. The main advantage of this confined geometry is the high attainable strain. Baaijens [6] compared the cross-slot flow with the flow around a cylinder and the contraction flow, and concluded that the highest strain is obtained in a sufficient large area in the cross-slot flow geometry. Higher strains can be obtained in fiber spinning experiments, but both thermal and deformation history are not defined well in this experiment. For example: the heat transfer of a crystallizing fiber is a complex problem both to accurately model and to measure. Moreover, the deformation history in extruder and die, used in these kind of experiments, is extremely difficult to determine, due to the complex flow field. A second advantage of the cross-slot flow device is the different type of materials that can be investigated. No special material properties are necessary, like the spinnable materials in fiber spinning experiments. A third advantage of this flow geometry is the free stagnation point obtained in the center of the cross, which is of practical use in the WAXS measurements. Stagnation flows are the only confined flows in which absorption by the walls can be circumvented (without considering the expensive option of transparent walls). The interesting region in shear flows is close to the wall, but this is not favorable for WAXS measurements, because part of the scattering is absorbed. A fourth advantage is the compactness of the cell, which results in controllable thermal gradients and thermal history.

\subsection{Conclusions}

\section{Characterization}

Detailed material characterization is essential to be able to investigate the relation between processing and crystallization. In this study two commercial polypropylenes, which differ in molecular weight, were characterized. Much attention was paid to the rheological characterization in shear and elongation. Both shear and elongational properties were fitted well by the extended Pompom model for both the materials investigated. The Leonov model, which serves as a basis for the $S_{\mathrm{J} 2}$ model, only gives a good description in shear. 
Moreover, nucleation and growth data were determined using a microscopy/hotstage set-up. A drawback of this method is the non-negligible thermal gradient that exists over the sight hole (with radius $1.3[\mathrm{~mm}]$ ) of the hot-stage, which is larger than $1.5\left[{ }^{\circ} \mathrm{C}\right]$. This is not important for the growth rate measurements, because the region of interest is small (radius $0.41[\mathrm{~mm}]$ ), resulting in a gradient of $0.3\left[{ }^{\circ} \mathrm{C}\right]$. However, it can have a large influence on the nucleation data, especially for the higher temperatures, because a large amount of nuclei are needed to determine a proper statistically mean value. Therefore, the number of nuclei were not counted at the sight hole, but on other spots that were directly in contact with the heating plate.

The nucleation and the growth data were incorporated in the Schneider rate equations to model the crystallization rate of DSC and small oscillatory shear experiments on a rheometer. Moreover, the spherulitical size obtained in light microscopy was compared with a numerical calculation of the structure. Comparing the crystallization rates between these methods, and the spherulite size, yielded the conclusion that a deviation in temperature was present, despite an accurate calibration. Moreover, one should be very careful with comparing the results of different experiments based on different methods (e.g. comparing space filling with modulus), because crystallization is quite sensitive to small temperature variations.

No unique relation was found between the space filling and the modulus. The interference of a trans-crystallization layer, is an explanation for this deviation. The thickness of this layer is of the same order as the gap height for the cone-plate geometry used. This can lead to an earlier increase of the modulus in time than one would observe without this layer. The slowest increase in the modulus was observed at a space filling of about 0.1-0.2 [-] for both materials.

The rheological behavior of both materials was investigated during and after short time shearing below the melting temperature. Only very small changes in the first normal stress difference during shear and the shear stress relaxation after cessation were observed.

\section{Cross slot flow cell}

Structure development in the cross-slot flow device was analyzed, experimentally and numerically. A qualitative agreement was found between the two experimental methods used (flow at low and at a high temperature prior to isothermal crystallization) and the numerical prediction. When a low flow rate was applied at the same temperature as the crystallization temperature, a highly oriented structure was found in the birefringence experiment, which survived up to 20 minutes after the cessation of flow. WAXS experiments showed that this structure corresponds with an orientation in the (110) and (130) reflection, corresponding to a fiber-like structure without branches.

For the same experiment, a large value of the space filling was predicted by the $S_{\mathrm{J} 2}$ model on the outflow centerline. However, it is questionable whether the, for these materials, unrealistic elongation thickening behavior influences the calculation of the number of nuclei. As an illustration may serve that a comparison between the Leonov and the Pompom model, without any coupling, shows a large difference in the first component of the Finger tensor, which serves as a basis for the creation of flow-induced nuclei. 
The influence of flow applied at a temperature above the melting temperature is not as pronounced as expected around the outflow centerline. Numerical simulations show that this is caused by the large shear gradients in the height and depth of the cell. The influence of both shear gradients are reduced if smaller flow times are applied, but still with the same residence time for particles close to the outflow centerline. Moreover, the shear gradient in the depth can be reduced if larger channel depth-to-height ratios are used. However, the maximum depth-to-height ratio that not influences the WAXS intensities is equal to 2.64 [-].

\subsection{Recommendations}

\section{Characterization}

For the investigations in the cross-slot flow cell, it is important to have proper elongational data. However, standard uniaxial elongational measurements are difficult to perform for these linear polymers. Therefore, it is recommended to determine elongation viscosities in confined dimensions like the cross-slot flow cell (see for example Schoonen [128], and for a quantitative analysis using the Pompom model, Verbeeten et al. [145]). A large depth-to-height ratio is needed for these measurements, e.g. larger than 8 [-], or a different optical method like the point-wise birefringence measurements should be employed. However, in this last case a full three-dimensional viscoelastic analysis should be performed.

\section{Cross slot set-up}

The flow cell was designed to create a strong flow (i.e. an elongational rate of about $\left.10\left[\mathrm{~s}^{-1}\right]\right)$ at a high temperature, and investigate crystallization at a low temperature. However, relaxation of the stresses plays an important role in these experiments. Its influence can be limited by using larger cooling rates or applying larger flow rates for a short time, also at temperatures below the melting temperature in the supercooled melt. Higher cooling rates can be obtained if the heat capacity of the set-up is reduced (smaller cell, shorter oil tubes), and/or if liquid nitrogen is used to cool the cell. Moreover, it is recommended to adjust the driving mechanism of the ring in order to perform also (high) flow experiments at temperatures below $\mathrm{T}_{\mathrm{m}}$.

A better control of the temperature history is necessary. Experience showed that the temperature history was difficult to reproduce. It was chosen not to control the temperature, but only to set the temperature. However, in order to compare the measurements, it is important that the temperature can be reproduced within half a degree Celsius. In case of quiescent experiments, a correction for differences in temperature is possible, using the Schneider rate equations. Consequently, the cell has to be calibrated accurately to allow for this comparison. In case of the flow experiments, a correction is difficult due to the many parameters involved, but it seems that the thermal sensitivity is less important for these experiments.

It is recommended to design a smaller cell with larger channel depth-to-height ratios, which is heated electrically and cooled with liquid nitrogen. Moreover, it is recommended to adjust the transmission between the motor and the ring to allow larger 
torques. The larger depth-to-height ratios are necessary to reduce the influence of the shear gradients along the optical path. Advantages of changing the heating and cooling of the cell are the more accurate control of the temperature due to the control loop between the heater and a thermocouple, and the faster attainable cooling rates due to the lower heat capacity of the set-up (no tubes, smaller cell).

Interesting is to investigate the relaxation after flow using a point-wise birefringence technique in the cross-slot flow cell at several temperatures. Possible changes in this relaxation behavior are related to the created flow-induced nuclei. 


\section{References}

[1] Aken, J.A. VAN AND JANESCHITZ-KRIEGL, H. New apparatus for the simultaneous measurement of stresses and flow birefringence in biaxial extension of polymer melts. Rheologica Acta, 20(5):419-432, 1980.

[2] Alfonso, G.C. AND ZiABICKI, A. Memory effects in isothermal crystallization. II. Isotactic polypropylene. Colloid and Polymer Science, 273:317-323, 1995.

[3] Andersen, P.G. AND CARR, S.H. Formation of bimodal crystal textures in polypropylene. Journal of Materials Science, 10:870-888, 1975.

[4] Anderson, P.D. Computational analysis of distributive mixing. Ph.D. thesis, Eindhoven University of Technology, 1999.

[5] AzZAM, R.M.A. Propagation of partially polarized light through anisotropic media with or without depolarization: A differential 4x4 matrix calculus. Journal of the Optical Society of America, 68:1756-1767, 1978.

[6] BAAIJENS, F.P.T. Quantitative predictions of viscoelastic polymer melt flow using the Pompom model, 2000. Dutch Society of Rheology meeting, Eindhoven, the Netherlands.

[7] BAAIJENS, J.P.W. Evaluation of constitutive equations for polymer melts and solutions in complex flows. Ph.D. thesis, Eindhoven University of Technology, 1994.

[8] Barnes, H.A., Hutton, J.F., And Walters, K. An Introduction to Rheology, volume 3 of Rheology Series. Elsevier, 1989.

[9] BAstiaAnsen, C.W.M. AND LeMSTRA, P.J. Melting behaviour of gelspun/drawn polyolefins. Makromolekulare Chemie, Macromolecular Symposia, 28:73-84, 1989.

[10] BAumgaertel, M. AND Winter, H.H. Interrelation between continuous and discrete relaxation time spectra. Journal of Non-Newtonian Fluid Mechanics, 44:1536, 1992.

[11] BEJAN, A. Heat Transfer. John Wiley and Sons, 1993.

[12] Bilsen, H.M.M. VAN, Fischer, H., KolnaAR, J.W.H., AND Keller, A. A temperature window of reduced flow resistance in polyethylene: In situ WAXS. Macromolecules, 28(25):8523-8527, 1995.

[13] Bird, R.B., Curtiss, C.F., Armstrong, R.C., And Hassager, O. Dynamics of Polymeric Liquids. John Wiley and Sons, 1987.

[14] Blundell, D.J., MaCKerRon, D.H., Fuller, W., Mahendrasingam, A., Martin, C., Oldman, R.J., Rule, R.J., And Riekel, C. Characterization of strain-induced crystallization of poly(ethylene terephthalate) at fast draw rates using synchrotron radiation. Polymer, 37(15):3303-3311, 1996.

[15] Blundell, D.J., Mahendrasingam, A., Martin, C., Fuller, W., MacKer- 
RON, D.H., HaRvie, J.L., Oldman, R.J., AND Riekel, C. Orientation prior to crystallisation during drawing of poly(ethylene terephthalate). Polymer, 41:77937802, 2000.

[16] Blundell, D.J., Oldman, R.J., Fuller, W., Mahendrasingam, A., MarTiN, C., MacKerron, D.H., Harvie, J.L., AND Riekel, C. Orientation and crystallization mechanisms during fast drawing of poly(ethylene terephthalate). Polymer Bulletin, 42:357-363, 1999.

[17] BORN, M. AND WOLF, E. Principles of Optics. Electromagnetic Theory of Propagation, Interference and Diffraction of Light. Cambridge University Press, 1999, seventh edition.

[18] Boutahar, K., Carrot, C., And Guillet, J. Polypropylene during crystallization from the melt as a model for the rheology of molten-filled polymers. Journal of Applied Polymer Science, 60:103-114, 1996.

[19] Boutahar, K., CARrot, C., AND Guillet, J. Crystallization of polyolefins from rheological measurements. Relation between the transformed fraction and the dynamic moduli. Macromolecules, 31:1921-1929, 1998.

[20] Bove, L., Nobile, M.R., Azzurri, F., And Alfonso, G.C. Shear-induced crystallization of isotactic polyolefins, 2001. PPS 17, Montreal, Canada.

[21] BRAS, W. Investigation crystallization on ESRF beam lines, 2000. COST meeting Vught, the Netherlands.

[22] Brǘckner, S., Meille, S.V., Petraccone, V., And Pirozzi, B. Polymorphism in isotactic polypropylene. Progress in Polymer Science, 16:361-404, 1991.

[23] Bushman, A.C. AND MCHugh, A.J. Transient flow-induced crystallization of a polyethylene melt. Journal of Applied Polymer Science, 64(11):2165-2176, 1997.

[24] CLARK, E.S. Unit Cell Information on some important polymers. In J.E. Mark, editor, Physical Properties of Polymers Handbook, AIP Series in Polymers and Complex Materials, chapter 30, pages 409-415. AIP Press, 1996.

[25] Corradini, P., Petraccone, V., De Rosa, C., And Guerra, G. On the structure of the quenched mesomorphic phase of isotactic polypropylene. Macromolecules, 19:2699-2703, 1986.

[26] Crowley, D.G., Frank, F.C., Mackley, M.R., And Stephenson, R.G. Localized flow birefringence of polyethylene oxide solutions in a four roll mill. Journal of Polymer Science: Polymer Physics Edition, 14:1111-1119, 1976.

[27] Cullity, B.D. Elements of X-ray Diffraction. Addison-Wesley Publishing Company, 1978, second edition.

[28] Dorset, D.L., McCourt, M.P., Kopp, S., Schumacher, M., OKihara, T., AND LOTZ, B. Isotactic polypropylene, $\beta$-phase: a study in frustration. Polymer, 39(25):6331-6337, 1998.

[29] Doufas, A.K., McHugh, A.J., And Miller, C. Simulation of melt spinning including $\mathrm{fl}$ 
properties of injection-moulded products. Progress in Polymer Science, 20:403-457, 1995.

[32] DUNLAP, P.N. AND LEAL, L.G. Dilute polystyrene solutions in extensional flows: birefringence and flow modification. Journal of Non-Newtonian Fluid Mechanics, 23:5-48, 1987.

[33] Duplay, C., Monasse, B., Haudin, J.-M., And Costa, J.-L. Shear-induced crystallization of polypropylene: Influence of molecular weight. Journal of Materials Science, 35:6093-6103, 2000.

[34] Eder, G. Crystallization in polymer processing: Modelling and experimentation. In L. Arkeryd, J. Bergh, P. Brenner, and R. Pettersson, editors, Progress in Industrial Mathematics at ECMI 98, pages 138-145. Teubner, 1999.

[35] EdER, G. AND JANESCHITZ-KRIEGL, H. Crystallization. In H.E.H. Meijer, editor, Processing of Polymers, volume 18 of Materials Science and Technology: A Comprehensive Treatment, chapter 5, pages 269-342. VCH, 1997.

[36] Eder, G., JANEschitz-KRIEGL, H., AND LiEdAuer, S. Crystallization process in quiescent and moving polymer melts under heat transfer conditions. Progress in Polymer Science, 15:629-714, 1990.

[37] Elias, H.-G. An Introduction to Polymer Science. VCH Publishers, 1997.

[38] FERRY, J.D. Viscoelastic properties of polymers. John Wiley and Sons, 1970, second edition.

[39] Field, J.E. The Properties of Diamond. Academic Press, 1979.

[40] FRANK, F.C. AND MACKLEY, M.R. Localized flow birefringence of polyethylene oxide solutions in a two roll mill. Journal of Polymer Science: Polymer Physics Edition, 14:1121-1131, 1976.

[41] FujiyAma, M. Higher order structure of injection-molded polypropylene. In J. Karger-Kocsis, editor, Polypropylene. Structure, Blends and Composites, volume 1, chapter 6, pages 167-204. Chapman and Hall, 1995.

[42] FujIYAMA, M. AND WAKINO, T. Structure of skin layer in injection-molded polypropylene. Journal of Applied Polymer Science, 35:29-49, 1988.

[43] Fuller, G.G. Optical Rheometry of Complex Fluids. Topics in Chemical Engineering. Oxford University Press, 1995.

[44] Fuller, G.G. AND MikKelsen, K.J. Note: Optical rheometry using a rotary polarization modulator. Journal of Rheology, 33:761-769, 1989.

[45] Gahleitner, M., Bachner, C., Ratajski, E., RohaczeK, G., And Neissl, W. Effects of the catalyst system on the crystallization of polypropylene. Journal of Applied Polymer Science, 73:2507-2515, 1999.

[46] Gahleitner, M., WolfschWenger, J. BaChner, C., And Bernreitner, K. Crystallinity and mechanical properties of PP-homopolymers as influenced by molecular structure and nucleation. Journal of Applied Polymer Science, 61:649$657,1996$.

[47] Gahleitner, M. And Wolfschwenger, J. Nucleating agents for semicrystalline thermoplastics. In Encyclopedia of Materials: Science and Technology. Pergamon, 2001. To be published.

[48] Galiatsatos, V., Neaffer, R.O., Sen, S., And Jeffrey Sherman, B. Refractive index, stress-optical coefficient, and optical configuration parameter of polymers. In J.E. Mark, editor, Physical Properties of Polymers Handbook, AIP Series 
in Polymers and Complex Materials, chapter 30, pages 409-415. AIP Press, 1996.

[49] Gallagher, P.K. Thermoanalytical methods. In E. Lifshin, editor, Characterization of Materials; Part I, volume 2A of Materials Science and Technology: A Comprehensive Treatment, chapter 7, pages 511-522. VCH, 1992.

[50] Gardner, K., Pike, E.R., Miles, M.J., Keller, A., And TanakA, K. Photoncorrelation velocimetry of polystyrene solutions in extensional flow fields. Polymer, 23:1435-1442, 1982.

[51] GefFroy, E. AND LEAL, L.G. Flow birefringence studies of a concentrated polystyrene solution in a two-roll mill. I. Steady flow and start-up of steady flow. Journal of Polymer Science: Part B: Polymer Physics, 30:1329-1349, 1992.

[52] Glatter, O. AND KRAtKY, O. Small Angle X-ray Scattering. Academic press, 1982.

[53] Gleissle, W. Druckverteilung im Spalt eines Kegel-Platte-Rheometers bei der Scherung viskoelastischer Flüssigkeiten mit hohen Schergeschwindigkeiten. Rheologica Acta, 15:305-316, 1976.

[54] Göschel, U., Swartjes, F.H.M, Peters, G.W.M., And Meijer, H.E.H. Crystallization in isotactic polypropylene melts during contraction flow: timeresolved synchrotron WAXD studies. Polymer, 41:1541-1550, 2000.

[55] Gottlieb, M. And MacosKo, C.W. The effect of instrument compliance on dynamic rheological measurements. Rheologica Acta, 21:90-94, 1982.

[56] Hammersley, A.P., Svensson, S.O., AND ThOMPSON, A. Calibration and correction of spatial distortions in 2D detector systems. Nuclear Instruments and Methods in Physics Research A, 346:312-321, 1994.

[57] Haudin, J.M. Optical studies of polymer morphology. In G.H. Meeten, editor, Processing of Polymers, chapter 4, pages 167-264. Elsevier Applied Science Publishers, 1986.

[58] Hecht, E. AND Zajac, A. Optics. Addison-Wesley, 1987, second edition.

[59] HePperle, J. AND SAITO, T. Tips for obtaining better results with the Rheometrics RME, 1997.

[60] Hobbs, J.K., McMaster, T.J., Miles, M.J., And Barham, P.J. Direct observations of the growth of spherulites of poly(hydroxybutyrate-co-valerate) using atomic force microscopy. Polymer, 39(12):2437-2446, 1998.

[61] HobBs, J.K. AND MileS, M.J. Direct observation of polyethylene shish-kebab crystallization using in situ atomic force microscopy. Macromolecules, 34:353-355, 2001.

[62] InKson, N.J., Mcleish, T.C.B., Harlen, O.G., AND Groves, D.J. Modelling the rheology of low density polyethylene in shear and extension with the multi-modal Pom-Pom constitutive equation, 1999. PPS 15, 's Hertogenbosch, the Netherlands.

[63] Janeschitz-Kriegl, H., Wippel, H., Lin, J.P., AND LiPP, M. On the kinetics of polymer crystallization in opposite-nozzle flow. Rheologica Acta, 40:248-255, 2001.

[64] JAY, F., HAUdIN, J.M., AND MONASSE, B. Shear-induced crystallization of polypropylenes: effect of molecular weight. Journal of Materials Science, 34:2089_ 2102, 1999.

[65] JERSCHOW, P. Crystallization of polypropylene. New experiments, evaluation methods 
and choice of material compositions. Ph.D. thesis, Johannes Kepler Universität, Linz, Austria, 1994.

[66] Jerschow, P. AND JANESChITZ-KRIEGL, H. On the development of oblong particles as precursors for polymer crystallization from shear flow: Origin of the so-called fine grained layers. Rheologica Acta, 35:127-133, 1996.

[67] JeRsCHOW, P. AND JANESCHITZ-KRIEGL, H. The role of long molecules and nucleating agents in shear induced crystallization of isotactic polypropylenes. International Polymer Processing XII, 1:72-77, 1997.

[68] Kalay, G., Allan, P., AND BeVIS, J. $\gamma$-Phase in injection moulded isotactic polypropylene. Polymer, 35(12):2480-2482, 1994.

[69] Kalay, G., Zhong, Z., Allan, P., AND Bevis, J. The occurrence of the $\gamma$ phase in injection moulded polypropylene in relation to the processing conditions. Polymer, 37(11):2077-2085, 1996.

[70] Karger-Kocsis, J., editor. Polypropylene. Structure, Blends and Composites: Structure and Morphology, volume 1. Chapman and Hall, 1995.

[71] Karger-Kocsis, J., VArgA, J., And Ehrenstein, G.W. Comparison of the fracture and failure behavior of injection-molded $\alpha$ - and $\beta$-polypropylene in high-speed three-point bending tests. Journal of Applied Polymer Science, 64:20572066, 1997.

[72] Keller, A. AND KOlnAAR, J.W.H. Flow-induced orientation and structure formation. In H.E.H. Meijer, editor, Processing of Polymers, volume 18 of Materials Science and Technology: A Comprehensive Treatment, chapter 4, pages 189-268. VCH, 1997.

[73] Keller, A., Müller, A.J., AND Odell, J.A. Entanglements in semi-dilute solutions as revealed by elongational flow studies. Progress in Colloid and Polymer Science, 75:179-200, 1987.

[74] KHAN, S.A. AND Prud'Homme, R.K. Melt rheology of filled thermoplastics. Reviews in Chemical Engineering, 4:205-270, 1987.

[75] KHANNA, Y.P. Rheological mechanism and overview of nucleated crystallization kinetics. Macromolecules, 26:3639-3643, 1993.

[76] Kumaraswamy, G., Issaian, A.M., And Kornfield, J.A. Shear-enhanced crystallization in isotactic polypropylene. 1. Correspondence between in situ rheo-optics and ex situ structure determination. Macromolecules, 32(22):7537$7547,1999$.

[77] Kumaraswamy, G., Verma, R.K., Issaian, A.M., Wang, P., Kornfield, J.A., YEH, F, HSIAO, B.S., AND OLLEY, R.H. Shear-enhanced crystallization in isotactic polypropylene. 2. Analysis of the formation of the oriented "skin". Polymer, 41:8931-8940, 2000.

[78] LARSON, R.G. Constitutive Equations for Polymer Melts and Solutions. Butterworths Series in Chemical Engineering. Butterworths Publishers, 1988.

[79] LARSON, R.G. The Structure and Rheology of Complex Fluids. Oxford University Press, 1999.

[80] Li, J.-M. AND BURGHARDT, W.R. Flow birefringence in axisymmetric geometries. Journal of rheology, 39(4):743-766, 1995.

[81] Liedauer, S., Eder, G., AND JANEschitz-KRIEGL, H. On the limitations of shear induced crystallization in polypropylene melts. International Polymer Pro- 
cessing XII, 3:243-250, 1995.

[82] Liedauer, S., Eder, G., JAneschitz-Kriegl, H., Jerschow, P., Geymayer, W., AND INGOLIC, E. On the kinetics of shear induced crystallization in polypropylene. International Polymer Processing XII, 3:236-244, 1993.

[83] LotZ, B. AND WitTMAnN, J.C. The molecular origin of lamellar branching in the $\alpha$ (monoclinic) form of isotactic polypropylene. Journal of Polymer Science: Part B: Polymer Physics, 24:1541-1558, 1986.

[84] Lotz, B., Wittmann, J.C., AND LovingeR, A.J. Structure and morphology of poly(propylenes): a molecular analysis. Polymer, 37(22):4979-4992, 1996.

[85] LOVINGER, A.J. Microstructure and unit-cell orientation in $\alpha$-polypropylene. Journal of Polymer Science: Polymer Physics Edition, 21:97-110, 1983.

[86] MackaY, M.E., DAJAN, A.M., Wippel, H., JANESChitZ-KRIEGL, H., AND LIPP, M. An approximate technique to determine elongation stresses in stagnation flow. Journal of Rheology, 39(1):1-14, 1995.

[87] Mackley, M.R., Moggridge, G.D., And SAquet, O. Direct experimental evidence for flow induced fibrous polymer crystallization occuring at a solid/melt interface. Journal of Materials Science, 35:1-7, 2000.

[88] Macosko, C.W. Rheology: Principles, Measurements and Applications. Advances in Interfacial Engineering Series. VCH Publishers, 1994.

[89] Macosko, C.W., OCANSEY, M.A., AND Winter, H.H. Steady planar extension with lubricated dies. Journal of Non-Newtonian Fluid Mechanics, 11:301-316, 1980.

[90] Magill, J.H. Crystallization of poly(tetramethyl-p-silphenylene)-siloxane (TMPS) polymers. Part II. Journal of Polymer Science: Part A-2, 5:89-99, 1967.

[91] Mahendrasingam, A., Blundell, D.J., Martin, C., Fuller, W., MacKerRON, D.H., HARVIE, J.L., OLDMAN, R.J., AND RIEKEL, C. Influence of temperature and chain orientation on the crystallization of poly(ethylene terephthalate) during fast drawing. Polymer, 41:7803-7814, 2000.

[92] Mahendrasingam, A., Martin, C., Fuller, W., Blundell, D.J., OldMAN, R.J., HARvie, J.L., MACKerRon, D.H., Riekel, C., AND ENGSTRÖM, P. Effect of draw ratio and temperature on the strain-induced crystallization of poly(ethylene terephthalate) at fast draw rates. Polymer, 40:5553-5565, 1999.

[93] Mahendrasingam, A., Martin, C., Fuller, W., Blundell, D.J., Oldman, R.J., MACKerRon, D.H., HARVIE, J.L., AND RIEKEL, C. Observation of a transient structure prior to strain-induced crystallization in poly(ethylene terephthalate). Polymer, 41:1217-1221, 2000.

[94] Masubuchi, Y., Watanabe, K., Nagatake, W., TAKimoto, J.-I., AND KOYAMA, K. Thermal analysis of shear induced crystallization by the shear flow thermal rheometer: isothermal crystallization of polypropylene. Polymer, 42:5023-5027, 2001.

[95] McHugH, A.J. Mechanisms of flow induced crystallization. Polymer Engineering and Science, 22(1):15-26, 1982.

[96] McHugh, A.J., GuY, R.K., AND TREe, D.A. Extensional flow-induced crystallization of a polyethylene melt. Colloid and Polymer Science, 271:629-645, 1993.

[97] MCLEISH, T.C.B. AND LARSON, R.G. Molecular constitutive equations for a class of branched polymers: The Pom-Pom polymer. Journal of Rheology, 42(1):81- 
$110,1998$.

[98] MEAD, D.W. Determination of molecular weight distributions of linear flexible polymers from linear viscoelastic material functions. Journal of Rheology, 38(6):1797-1827, 1994.

[99] Meille, S.V., Ferro, D.R., BrüCKner, S., Lovinger, A.J., And PAdden, F.J. Structure of $\beta$-isotactic polypropylene: a long-standing structural puzzle. Macromolecules, 27:2615-2622, 1994.

[100] Miles, M.J. AND KelleR, A. Conformational relaxation time in polymer solutions by elongational flow experiments: 2. Preliminaries of further developments: chain retraction; identification of molecular weight fractions in a mixture. Polymer, 21:1295-1298, 1980.

[101] MonAsse, B. Polypropylene nucleation on a glass fibre after melt shearing. Journal of Materials Science, 27:6047-6052, 1992.

[102] MonAsse, B. Nucleation and anisotropic crystalline growth of polyethylene under shear. Journal of Materials Science, 30:5002-5012, 1995.

[103] MONASSE, B. AND HAUDIN, J.M. Growth transition and morphology change in polypropylene. Colloid and Polymer Science, 263:822-831, 1985.

[104] MonKs, A.W., White, H.M., AND BAssetT, D.C. On shish-kebab morphologies in crystalline polymers. Polymer, 37:5933-5936, 1996.

[105] Nadella, H.P., Henson, H.M., SPruiell, J., And White, J.L. Melt spinning of isotactic polypropylene: structure development and relationship to mechanical properties. Journal of Applied Polymer Science, 21:3003-3022, 1977.

[106] NagATAKe, W., TAKAhashi, T., MASUbUCHI, Y., TAKIMOTO, J.-I., AND KOYAMA, K. Development of shear flow thermal rheometer for direct measurement of crystallization fraction of polymer melts under shear deformation. Polymer, 41:523-531, 2000.

[107] NATIONAL INSTRUMENTS. PCI E Series and BNC-2090 user manual (digital version), 2000.

[108] Norton, D.R. AND Keller, A. The spherulitic and lamellar morphology of melt-crystallized isotactic polypropylene. Polymer, 26:704-716, 1985.

[109] Pahl, M., Gleissle, W., AND LAun, H.M. Praktische Rheologie der Kunststoffe und Elastomere. VDI-Verlag GmbH, 1995.

[110] PATTAMAPROM, C., LARSON, R.G., AND DYKE, T.J. VAN. Quantitative predictions of linear viscoelastic rheological properties of entangled polymers. Rheologica Acta, 39:517-531, 2000.

[111] PETERS, G.W.M. Thermorheological modelling of viscoelastic materials. In J.F. Dijksman and G.D.C. Kuiken, editors, IUTAM Symposium on Numerical Simulation of Non-Isothermal Flow of Viscoelastic Liquids; Proceedings of an IUTAM Symposium held in Kerkrade, The Netherlands, 1-3 November 1993, volume 28 of Fluid Mechanics and its Applications, pages 21-35. Kluwer Academic Publishers, 1995.

[112] Peters, G.W.M. The Pom-Pom model, 2001. Lecture notes.

[113] Peters, G.W.M. And BAAijEns, F.P.T. Modelling of non-isothermal viscoelastic flows. Journal of Non-Newtonian Fluid Mechanics, 68:205-224, 1997.

[114] Pogodina, N.V., SiddiqueE, S.K., Egmond, J.W. VAN, AND WinTer, H.H. Correlation of rheology and light scattering in isotactic polypropylene during early stages of crystallization. Macromolecules, 32:1167-1174, 1999. 
[115] Pogodina, N.V. AND Winter, H.H. Polypropylene crystallization as a physical gelation process. Macromolecules, 31:8164-8172, 1998.

[116] Pogodina, N.V., Winter, H.H., AND SRINIVAS, S. Strain effects on physical gelation of crystallizing isotactic polypropylene. Journal of Polymer Science: Part B: Polymer Physics, 37:3512-3519, 1999.

[117] RAMSEY III, J.C., FRICKE, A.L., AND CASKEY, J.A. Thermal conductivity of polymer melts. Journal of Applied Polymer Science, 17:1597-1605, 1973.

[118] RATAjSKI, E. AND JANESCHITZ-KRIEGL, H. How to determine high growth speeds in polymer crystallization. Colloid and Polymer Science, 274:938-951, 1996.

[119] Ryan, A.J., FAirclough, J.P.A., Terrill, N.J., Olmsted, P.D., AND PoON, W.C.K. A scattering study of nucleation phenomena in polymer crystallisation. Faraday Discussions, 112:13-29, 1999.

[120] SAMON, J.M., SCHUltZ, J.M., AND HsiAO, B.S. Morphological changes during the annealing of polybutene-1 fiber. Macromolecules, 34:2008-2011, 2001.

[121] SAmon, J.M., Schultz, J.M., Hsiao, B.S., Seifert, S., Stribeck, N., Gurke, I., COllins, G., AND SAW, C. Structure development during the melt spinning of polyethylene and poly(vinylidene fluoride) fibers by in situ synchrotron small- and wide angle X-ray scattering techniques. Macromolecules, 32(24):81218132, 1999.

[122] SAmON, J.M., Schultz, J.M., HsiaO, B.S., Wu, J., AND KHOT, S. Structure development during melt spinning and subsequent annealing of polybutene-1 fibers. Journal of Polymer Science: Part B: Polymer Physics, 38:1872-1882, 2000.

[123] Samon, J.M., Schultz, J.M., Wu, J., HsiaO, B.S., YeH, F., AND Kolb, R. Study of the structure development during the melt spinning of nylon 6 fiber by on-line wide angle synchrotron X-ray scattering techniques. Journal of Polymer Science: Part B: Polymer Physics, 37:1277-1287, 1999.

[124] SAMUELS, R.J. Quantitative structural characterization of the melting behavior of isotactic polypropylene. Journal of Polymer Science: Polymer Physics Edition, 13:1417-1446, 1975.

[125] SAMUELS, R.J. AND YANG YEE, R. Characterization of the structure and organization of $\beta$-form crystals in Type III and Type IV isotactic polypropylene spherulites. Journal of Polymer Science: Part A-2, 10:385-432, 1972.

[126] SCHNEIDER, W., BERGER, J., AND KÖPPL, A. Non-isothermal crystallization of polymers: Application of Rate Equations. In Physico-Chemical Issues in Polymers, pages 1043-1054. Technomic Publ. Co., 1993.

[127] Schneider, W., KÖPPL, A., AND BERGER, J. Non-isothermal crystallization, crystallization of polymers. International Polymer Processing II, 3:151-154, 1988.

[128] SCHOONEN, J.F.M. Determination of rheological constitutive equations using complex flows. Ph.D. thesis, Eindhoven University of Technology, 1998.

[129] SChOONEN, J.F.M., SwartJes, F.H.M., PETERS, G.W.M., AND BAAIJENS, F.P.T. A 3D numerical/experimental study on a stagnation flow of a polyisobutylene solution. Journal of Non-Newtonian Fluid Mechanics, 79(2):529-562, 1998.

[130] SCHOTT GLASWERKE. Optisches Glas, 1993.

[131] Schultz, J.M., HsiaO, B.S., AND SAMON, J.M. Structure development during the early stages of polymer melt spinning by in-situ synchrotron X-ray tech- 
niques. Polymer, 41:8887-8895, 2000.

[132] Schultz, J.M. AND PetermanN, J. Transmission electron microscope observations of fibrillar-to-lamellar transformations in melt-drawn polymers - I. Isotactic polypropylene. Colloid and Polymer Science, 262:294-300, 1984.

[133] SEGAL, A. Manual SEPRAN package, 1992. Ingenieursburo SEPRA, Leidschendam, The Netherlands.

[134] Somani, R.H., Hsiao, B.S., Nogales, A., SRinivas, S., Tsou, A.H., Sics, I, BAltA-CAllejA, F.J., AND EZQUerRA, T.A. Structure development during flow induced crystallization of i-PP: in situ small angle X-ray scattering study. Macromolecules, 33:9385-9394, 2000.

[135] Stocker, W., Schumacher, M., Graff, S., Thierry, A., WitTmann, J.-C, AND LOTZ, B. Epitaxial crystallization and AFM investigation of a frustrated polymer structure: isotactic poly(propylene), $\beta$ phase. Macromolecules, 31:807814, 1998.

[136] SWARTJES, F.H.M. A method for the evaluation of 3D viscoelastic flows. Master's thesis WFW 96.157, Eindhoven University of Technology, 1996.

[137] SWARTJES, F.H.M. Design, development and realization of a cross-slot flow device for investigation of structure development in elongational flow of melts. MTD thesis WFW 99.014, Eindhoven University of Technology, 1999.

[138] TAS, P.P. Film blowing: from polymer to product. Ph.D. thesis, Eindhoven University of Technology, 1994.

[139] Terrill, N.J., FAirclough, P.A., TOWNS-Andrews, E., KomanscheK, B.U., YOUNG, R.J., AND RYAN, A.J. Density fluctuations: the nucleation event in isotactic polypropylene crystallization. Polymer, 39(11):2381-2385, 1998.

[140] Tribout, C., Monasse, B., AND Haudin, J.M. Experimental study of shearinduced crystallization of an impact polypropylene copolymer. Colloid and Polymer Science, 274:197-208, 1996.

[141] VARGA, J. Crystallization, melting and supermolecular structure of isotactic polypropylene. In J. Karger-Kocsis, editor, Polypropylene. Structure, Blends and Composites, volume 1, chapter 3, pages 56-115. Chapman and Hall, 1995.

[142] VARGA, J. AND KARGER-KOCSIS, J. Rules of supermolecular structure formation in sheared isotactic polypropylene melts. Journal of Polymer Science: Part B: Polymer Physics, 34:657-670, 1996.

[143] Verbeeten, W.M.H., Peters, G.W.M., And BaAijens, F.P.T. Differential constitutive equations for polymer melts: The extended Pom-Pom model. Journal of Rheology, 45(4):823-844, 2001.

[144] Verbeeten, W.M.H., Peters, G.W.M., and BaAijens, F.P.T. The extended Pom-Pom model for complex polymer melt flows: a quantitative description, 2001. PPS 17, Montreal, Canada.

[145] Verbeeten, W.M.H., Peters, G.W.M., And BaAijens, F.P.T. Viscoelastic analysis of complex polymer melt flows using the extended Pom-Pom model. Submitted to the Journal of Non-Newtonian Fluid Mechanics, 2001.

[146] Verma, R.K. AND HsiaO, B.S. Some new insights into the crystallization and melting mechanism in semicrystalline semistiff polymers. Trends in Polymer Science, 4:312-319, 1996.

[147] Vleeshouwers, S. AND Meijer, H.E.H. A rheological study of shear induced 
crystallization. Rheologica Acta, 35:391-399, 1996.

[148] Wang, Z.-G., HsiaO, B.S., Sirota, E.B., Agarwal, P., ANd SRinivas, S. Probing the early stages of melt crystallization in polypropylene by simultaneous small- and wide-angle X-ray scattering and laser light scattering. Macromolecules, 33:978-989, 2000.

[149] WANG, Z.-G., HsiaO, B.S., SirotA, E.B., AND SRINIVAS, S. A simultaneous small- and wide-angle X-ray Scattering study of the early stages of melt crystallization in polyethylene. Polymer, 41:8825-8832, 2000.

[150] White, F.M. Viscous Fluid Flow, pages 119-125. McGraw-Hill, 1974.

[151] White, H.M. AND BASSETT, D.C. On variable nucleation geometry and segregation in isotactic polypropylene. Polymer, 38(22):5515-5520, 1997.

[152] White, H.M. AND BASSETT, D.C. On row structures, secondary nucleation and continuity in $\alpha$-polypropylene. Polymer, 39(14):3211-3219, 1998.

[153] WHITE, J.L. AND CAKMAK, M. Orientation development and crystallization in melt spinning of fibers. Advances in Polymer Technology, 6(3):295-337, 1986.

[154] Wu, C.-M., CHEN, M., AND KARGER-Kocsis, J. The role of metastability in the micromorphologic features of sheared isotactic polypropylene melts. Polymer, 40:4195-4203, 1999.

[155] ZOETELIEF, W.F. On the numerical simulation of the multilayer injection moulding process. Technical Report WFW 92.100, Institute for Continuing Education, Eindhoven University of Technology, 1992.

[156] ZuidemA, H. Flow induced crystallization of polymers. Application to injection moulding. Ph.D. thesis, Eindhoven University of Technology, 2000.

[157] Zuidema, H., Peters, G.W.M., And Meijer, H.E.H. Development and validation of a recoverable strain based model for flow-induced crystallization of polymers. Macromolecular Theory and Simulations, 10(5):447-460, 2001. 


\section{Appendix A}

\section{Details of components and devices}

In this appendix all product details of the components/devices used are given.

\section{A.1 Cross-slot flow cell}

\section{Temperature control}

\begin{tabular}{|l|l|l|l|}
\hline Device & Manufacturer & Type & Remarks \\
\hline \hline Oil bath & LAUDA & K 6 KP & $\mathrm{Q}=24$ litre/min; V=7.5 litre \\
& LAUDA & UB 20 & Q=22 litre/min; V=18 litre \\
\hline Metal tube & LAUDA & MC50 M16 1 & insulated tube \\
\hline Oil bath fluid & Dow Corning & 550 & \\
\hline Valve & Klinger & ABSV 18 & multi-stage cock with 0-position \\
\hline Rotator & Bar GmbH & GTD 55x90 & \\
\hline Pneumatic valve & Airtec & M 05 510-HN & $5 / 2$ air valve \\
\hline Quick coupling & TEMA & 2520 V 1/4 Bi/dr & $\begin{array}{l}\text { male } \\
\text { female }\end{array}$ \\
& TEMA & $2510 \mathrm{~T} 1 / 4 \mathrm{Bi} / \mathrm{dr}$ & \multicolumn{2}{|l}{} \\
\hline
\end{tabular}

Table A.1: Components used to control the temperature history of the cross-slot flow cell. The maximum temperature and heating power is equal to $300\left[{ }^{\circ} \mathrm{C}\right]$ and $3.0[\mathrm{~kW}]$ respectively for both baths. Q: max. flow; V: volume.

\section{Temperature measurement}

Temperature in the cross-slot cell is measured and recorded in time by two thermocouples. These thermocouples (Hasco) were connected (blue wire number 2; red wire number 3) with a signal conditioner (Keithley). The signals were transferred to a shielded BNC adapter chassis, which was connected to a 16 bit I/O board using a shielded cable (all three components were made by National Instruments). The reference single-ended (RSE) input mode (the ground is the reference signal) and the unipolar input range (0-10 [V]) are used, (accuracy: $152.59[\mu \mathrm{V}])$. This accuracy can be in- 
creased by using a software programmable gain of 2 or 5 (National Instruments [107]). Data were visualized and saved in the program Virtual Bench (National Instruments).

The thermocouple was calibrated by putting it in a oil bath at six different temperatures $\left(100,120,140,160,180\right.$ and $\left.200\left[{ }^{\circ} \mathrm{C}\right]\right)$. The linear relation between voltages and temperatures was determined by fitting the measured points.

\begin{tabular}{|l|l|l|l|}
\hline Device & Manufacturer & Type & Remarks \\
\hline \hline Thermocouple (type J) & Hasco & Z1295 & \\
\hline Thermocouple input module & Keithley & MB37-J-01 & $\begin{array}{l}\text { output: 0-5 [V] } \\
\left.-100 \text { to 760 [ }{ }^{\circ} \mathrm{C}\right]\end{array}$ \\
\hline Shielded BNC adapter chassis & National Instruments & BNC2090 & \\
\hline Shielded cable & National Instruments & SH6868EP & \\
\hline 16 bit I/O board & National Instruments & PCI-MIO-16XE & \\
\hline
\end{tabular}

Table A.2: Components used to measure the temperature in the cross-slot flow cell.

\section{Displacement measurement}

A Lucas Schaevitz DC-operated Linear Variable Differential Transformer (LVDT) is used to measure the displacement of the ring of the cell. The sensitivity of the device is equal to $4.9130[\mathrm{~mm} / \mathrm{V}]$. The voltages are reduced with a factor half to get in the range of $10[\mathrm{~V}]$ in order to measure the full displacement of the ring (about $94.7[\mathrm{~mm}]$ ) within the measuring range of the I/O board.

\section{Positioning}

Position of the flow cell can be defined with two translation stages which are connected with a motion controller/driver (Newport). Since ten meter long cables are used to connect the driver with the stages, an amplifier has to be used between cable and motor. The driver can communicate with a PC through a RS-232C interface.

\begin{tabular}{|l|l|l|l|}
\hline Device & Manufacturer & Type & Remarks \\
\hline \hline Horizontal translation stage & Newport & M-MTM200PP.1 & $\begin{array}{l}0.1 \text { micron step } \\
\text { travel range: } 200 \text { [mm] }\end{array}$ \\
\hline Vertical translation stage & Newport & UZM160PP.05 & $\begin{array}{l}0.05 \text { micron step } \\
\text { travel range: } 9 \text { [mm] }\end{array}$ \\
\hline Motion Controller/Driver & Newport & MM4005 & \\
\hline Motor Cable & Newport & E1110B & \\
\hline 5 V amplifier for motor & Newport & E1050B & Necessary for E1110B \\
\hline
\end{tabular}

Table A.3: Components to position the cell. 


\section{A.2 Microscopy}

Details of the hot-stage microscopy set-up, used in the nucleation and growth experiments, are described in Table A.4. The hot-stage was mounted on the microscope using a special mounting ring. The relation between objectives and pixel size is given in Table A.5.

\begin{tabular}{|l|l|l|l|}
\hline Device & Manufacturer & Type & Remarks \\
\hline \hline Microscope & Zeiss & Axiolab & \\
\hline Hot-stage & Linkam & LTS 350 & \\
\hline Heating device & Linkam & TP 93 & \\
\hline Cooling pump & Linkam & LNP 1 & liquid nitrogen \\
\hline Color video camera & Sony & DXC-151 AP & 756×581 pixels \\
\hline Video recorder & Panasonic & AG 6720 & time-lapsed \\
\hline
\end{tabular}

Table A.4: Devices used in the microscopy set-up to determine growth.

\begin{tabular}{|l|r|}
\hline Objective & Pixel size [m] \\
\hline \hline Achroplan $4 \times$ & $4.3197 \cdot 10^{-6}$ \\
\hline Achroplan $10 \times$ & $1.7308 \cdot 10^{-6}$ \\
\hline LD Achroplan $20 \times$ & $8.5455 \cdot 10^{-7}$ \\
\hline Achroplan $40 \times$ & $4.3355 \cdot 10^{-7}$ \\
\hline
\end{tabular}

Table A.5: Relation between objective and pixel size for the Zeiss microscope in combination with the Sony video camera.

\section{A.3 Birefringence}

The components for the field-wise birefringence experiments (see also Figure 6.4) are given in Table A.6. The tilt and the rotation angle of the stereo microscopy can be adjusted with a tilt/rotation stage (M-PO80BL). The position of the microscopy in beam direction can be adjusted by a precision translation stage (M-UMR). 


\begin{tabular}{|l||l|l|l|}
\hline Device & Manufacturer & Type & Remarks \\
\hline \hline Laser & Uniphase & 1125 P & $\lambda=632.8[\mathrm{~nm}]$ \\
\hline Beam expander & Edmund Scientific & A61, 384 & $10 \times$ expander \\
\hline Linear polarizer & Meadowlark Optics & DPM-200-VIS-1 & \\
\hline Quarter wave retarder & Meadowlark Optics & A103 & $\lambda=632.8[\mathrm{~nm}]$ \\
\hline Color video camera & Panasonic & WV-CD130 & \\
\hline Stereo microscope & Zeiss & SV-11 & \\
\hline \hline Tilt/rotation stage & Newport & M-PO80BL & \\
\hline Translation stage & Newport & M-UMR 12.63 & $\begin{array}{l}+ \text { micrometer } \\
\text { BM25.63 }\end{array}$ \\
\hline Laboratory jack & Newport & 271 & \\
\hline
\end{tabular}

Table A.6: Components for the field-wise birefringence experiments. $\lambda=$ wave length. 


\section{Appendix B}

\section{Temperature gradient in hot-stage}

A hot-stage/microcopy set-up was used in Chapter 4 to determine the number of nuclei and growth rate as a function of temperature. The temperature gradients obtained in a hot-stage can be quite large. A gradient of about $7\left[{ }^{\circ} \mathrm{C}\right]$ was found by Hobbs and Miles [61], who used a hot-stage together with atomic force microscopy (AFM).

In the experiments described in this thesis, a sample was positioned between two glass plates. At the hole of the heating plate, a temperature gradient will arise, because the heat transfer between glass with surrounding air is more pronounced than the low conduction within the glass. The measurement of this gradient is described in this appendix.

Three different liquid crystalline polymers, with a well defined melting temperature $T_{m}$, were used to determine the temperature gradient in the hot-stage (Table B.1). Crystals were positioned between two glass slides, having a thickness of about 0.146 [mm] each. First, these crystals were melted to obtain a well defined sample, having a thickness of about $10[\mu \mathrm{m}]$. Next, the melting behavior of this sample was recorded using a heating rate of $0.1\left[{ }^{\circ} \mathrm{C} / \mathrm{min}\right]$.

\begin{tabular}{|l||r||r|r|r|r|}
\hline name & $\mathrm{T}_{\mathrm{m}}\left[{ }^{\circ} \mathrm{C}\right]$ & $\mathrm{A}$ & $\mathrm{B}$ & $\mathrm{C}$ & $\Delta \mathrm{T}_{\text {mean }}\left[{ }^{\circ} \mathrm{C}\right]$ \\
\hline \hline Acetanilid & 114.5 & -5.90 & -2.43 & 2.70 & 2.59 \\
\hline Phenacetin & 134.5 & -7.68 & -2.83 & 4.55 & 4.40 \\
\hline Benzanilid & 163 & -1.27 & -4.88 & 5.20 & 5.09 \\
\hline
\end{tabular}

Table B.1: Fit parameters of the measured temperature difference between sample and hotstage for three different liquid crystalline polymers.

An example of a recorded image is shown in Figure B.1(a). In the center of the hole, a region is visible that is not melted. The radius of this region was determined in time. One image per minute was captured from the video tape and saved as bitmaps, using Adobe Premiere software. The radius of the circular region was determined in Matlab (The Mathworks, Inc.; see for example Figure B.1(b)). The relation between radius and temperature is also known, because the heating rate was prescribed. The difference between the hot-stage temperature $T_{h s}$ and the melting temperature $T_{m}$ is plotted against radius in Figure B.2(a). 
The measurements were fitted by a quadratic function

$$
\Delta T=A r^{2}+B r+C
$$

and its coefficients are given in Table B.1. Coefficient C, which describes the temperature difference if all material is melted $(\mathrm{r}=0)$, becomes larger at higher temperatures.

Temperature in the experiments should be corrected for the temperature gradient in the sample. However, the temperature depends on position, as shown above. Therefore a mean temperature $T_{\text {mean }}$ was defined as the temperature averaged by the area in a circle with radius $R$. It can be derived that the $\Delta \mathrm{T}_{\text {mean }}$ for this area is described by

$$
\Delta T_{\text {mean }}=\frac{1}{2} A R^{2}+\frac{2}{3} B R+C
$$

The radius $\mathrm{R}$ was chosen equal to half the diagonal of an image and was calculated by combining the image size $(768 \times 576$ pixels) and the pixel-size (Table A.5). A $20 \times$ objective was used in the experiments to determine the growth rate. This results into an 'image radius' of $0.41[\mathrm{~mm}]$. The corresponding $\Delta \mathrm{T}_{\text {mean }}$ are given in Table B.1.

The relation between the averaged temperatures $\mathrm{T}_{\text {mean }}$ and the hot-stage temperature $T_{\text {hs }}$ was used to correct the temperature in the growth rate experiments. This relation is shown in Figure B.2(b) and described by

$$
T_{\text {mean }}=0.952 \cdot T_{h s}+2.71
$$

For example, setting the temperature of the hot-stage equal to $140\left[{ }^{\circ} \mathrm{C}\right]$ gives an averaged sample temperature of $136.0\left[{ }^{\circ} \mathrm{C}\right]$ ! 


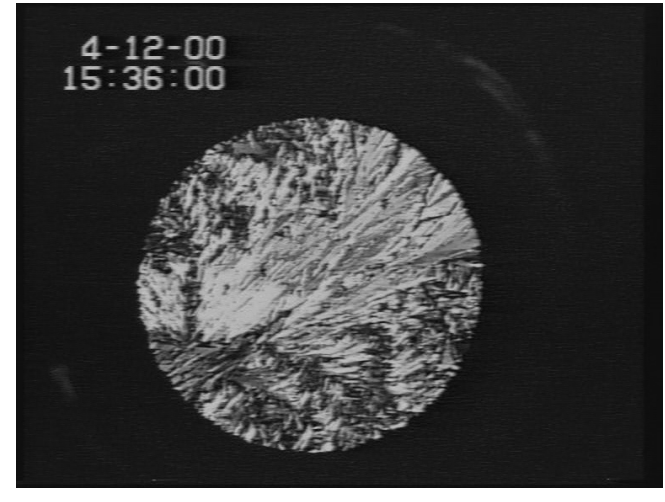

(a)

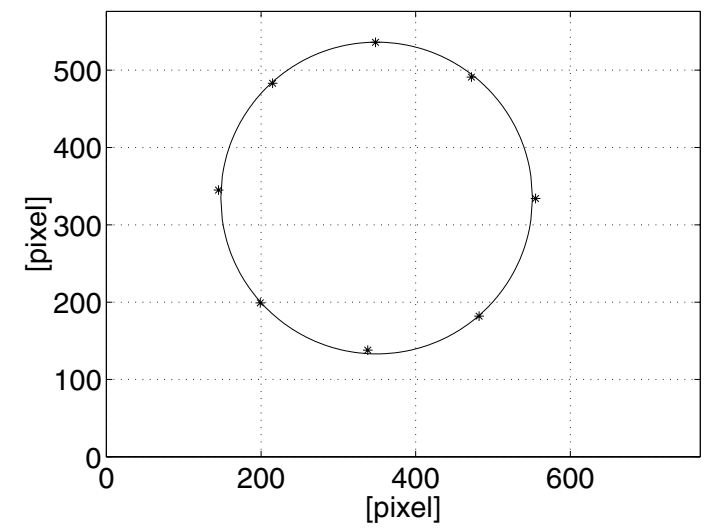

(b)

Figure B.1: (a) Phenacetin at $\mathrm{T}_{\mathrm{hs}}=138.2\left[{ }^{\circ} \mathrm{C}\right]$ with (b) corresponding circular fit. Determined radius was equal to $0.53[\mathrm{~mm}]$. Notice that pixel $(1,1)$ is positioned in the upper left corner of the image.

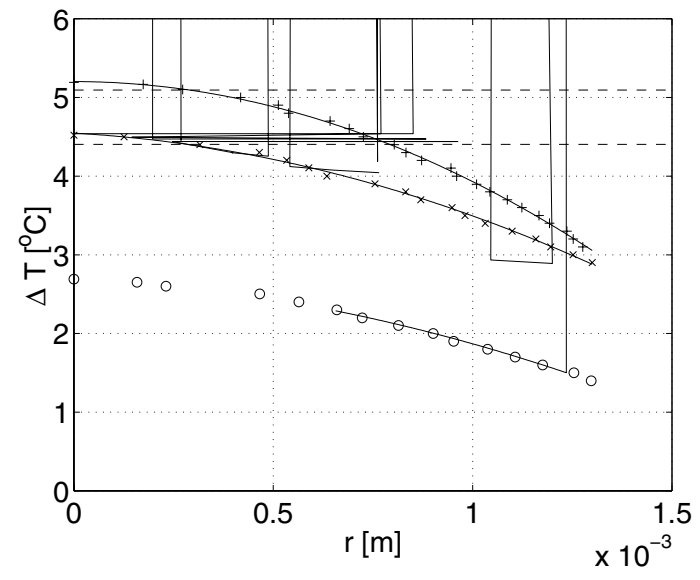





\section{Appendix C}

\section{Velocities and velocity gradients in the cross-slot flow}

Most important velocities and velocity gradients in the cross-slot flow are shown in this appendix. A flow rate of $1.85 \cdot 10^{-8}\left[\mathrm{~m}^{3} / \mathrm{s}\right]$ was applied at $200\left[{ }^{\circ} \mathrm{C}\right]$ for DSM13E10 and Daplen KS10. The position and velocity in the cross-slot flow are normalized with $1[\mathrm{~mm}](\mathrm{L})$ and $1[\mathrm{~mm} / \mathrm{s}](\mathrm{U})$, respectively.

Velocities and velocity gradients at surface $\mathrm{z}=0[\mathrm{~mm}]$ are shown in Figure C.1 and C.2 for DSM13E10 and Daplen KS10, respectively. The shear thinning behavior of DSM13E10 is more pronounced. The large values for the velocity gradients close to the corner are caused by the singularity in the corner point.

Velocities and velocity gradients in the depth of the flow cell for Daplen KS10 are shown in Figure C.3. It is clear that three-dimensional effects cannot be neglected, because both the elongational components $(\mathrm{du} / \mathrm{dx}$ and $\mathrm{dv} / \mathrm{dy})$ and the shear component $\mathrm{dv} / \mathrm{dz}$ are varying in depth. 


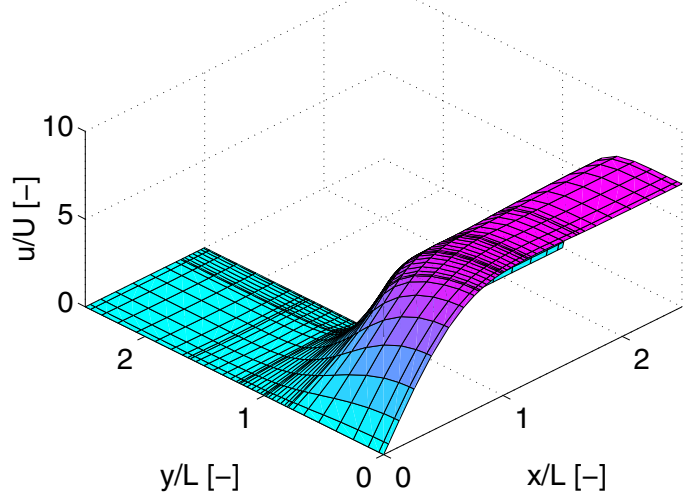

(a)

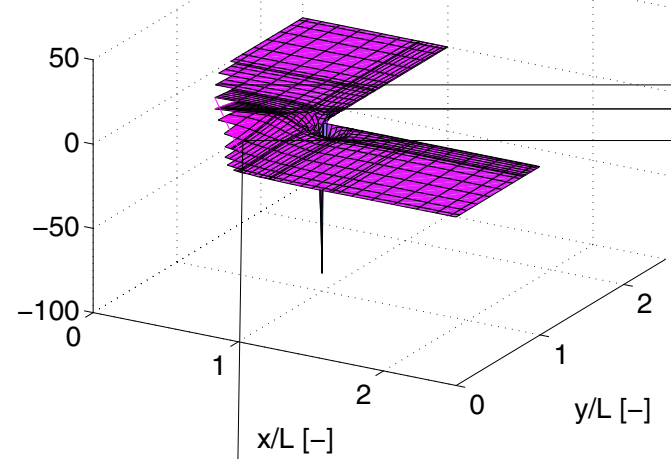

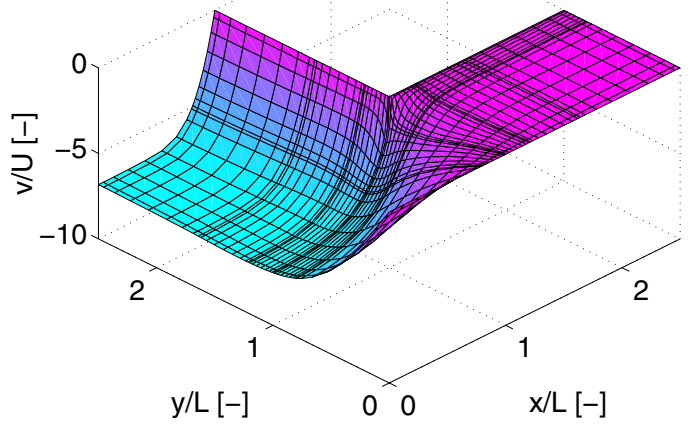

(b) 


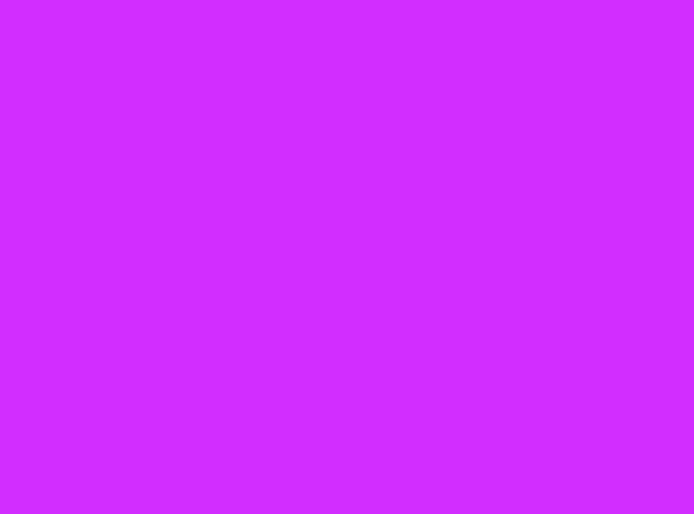

(a)

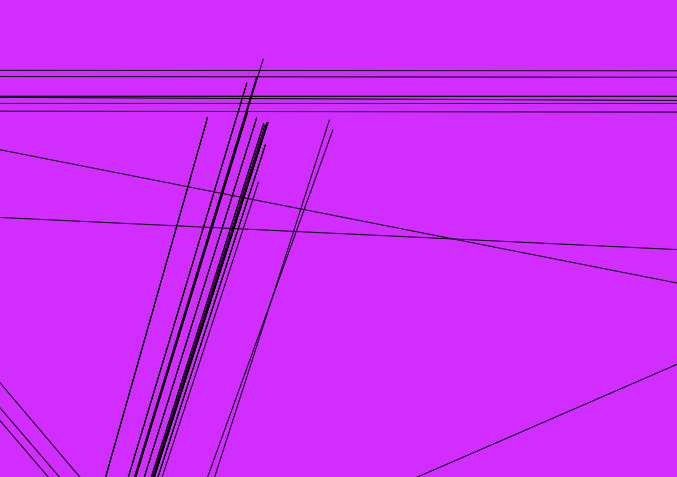

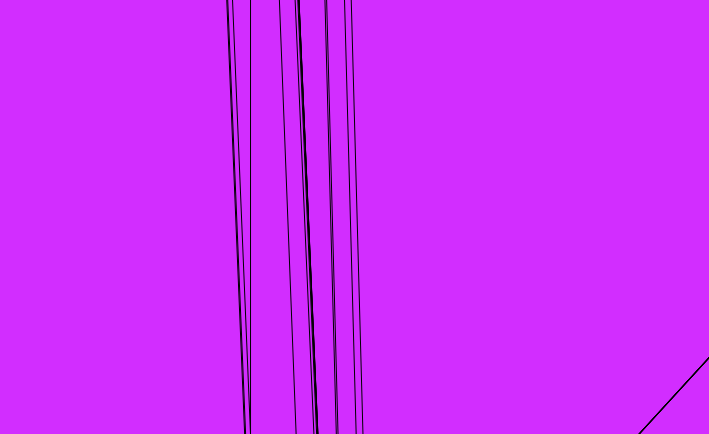

(b) 


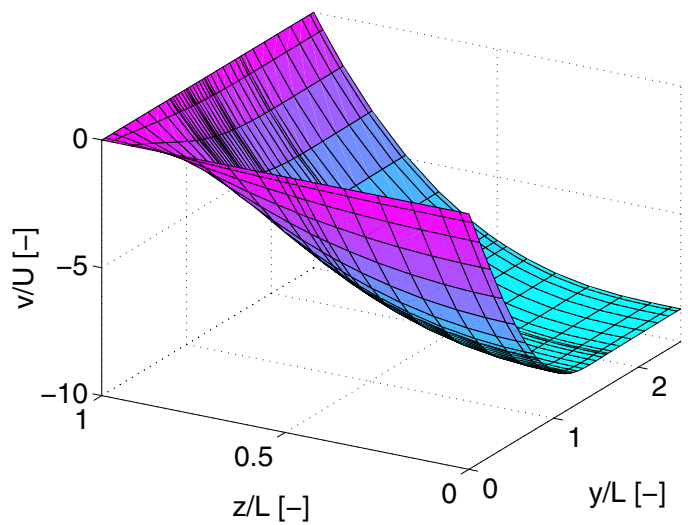

(a)

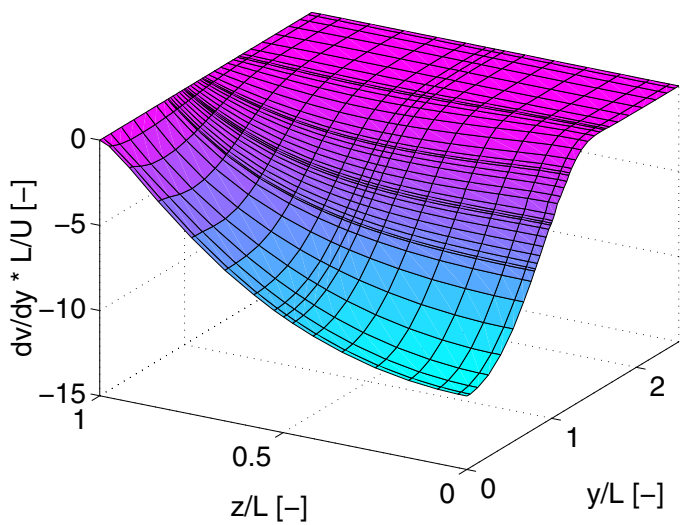

(c)

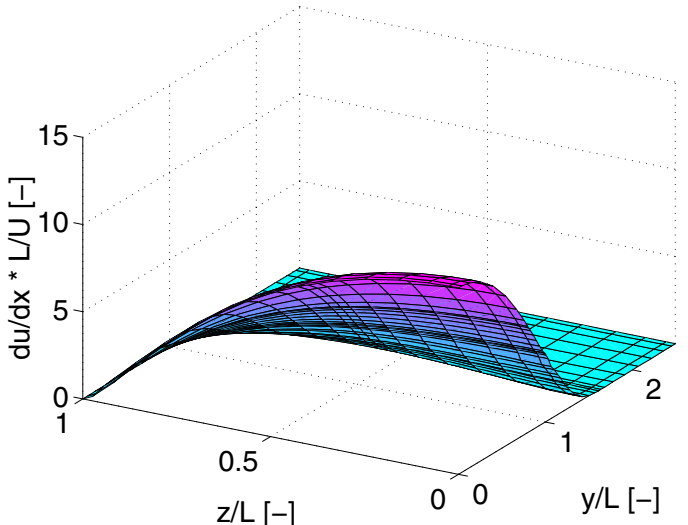

(b)

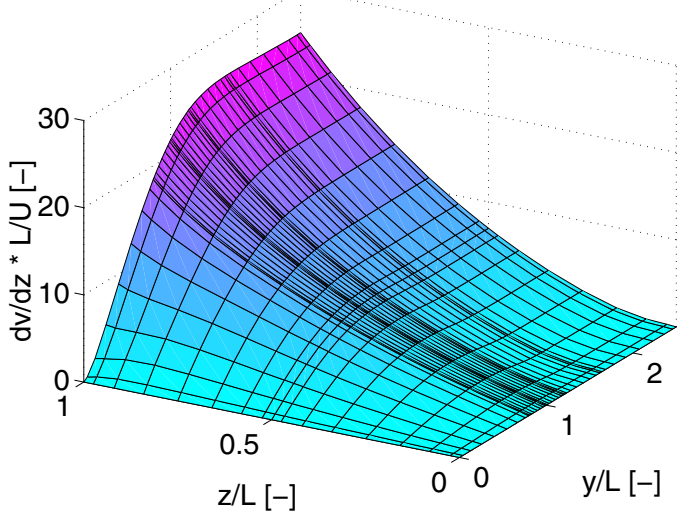

(d)

Figure C.3: Velocity and velocity gradients in the cross-slot flow cell at surface $x=0[\mathrm{~mm}]$ for Daplen KS10, applying a flow rate of $1.85 \cdot 10^{-8}\left[\mathrm{~m}^{3} / \mathrm{s}\right]$ at $200\left[{ }^{\circ} \mathrm{C}\right] . \mathbf{( a )} \mathrm{v} ;$ (b) $\mathrm{du} / \mathrm{dx}$; (c) $d v / d y ;(d) d v / d z$. 


\section{Appendix D}

\section{Material properties of the windows}

In this appendix, the most important properties of the two window materials are given. Second, the temperature gradients near the window are determined. Finally, the alignment of the microscope in the field-wise birefringence measurement is discussed. Determination of the window thickness is described in Swartjes [137, Appendix B].

\section{D.1 Properties of diamond and Schott SF57}

Depending on the amount of nitrogen and boron, several types of diamond exist. Type IIa diamonds, which have a low nitrogen concentration, were provided by Drukker International (Cuijk, the Netherlands). The optical glass SF57 was provided by Schott. Properties of both materials are given in Table D.1.

\begin{tabular}{|l|l||r|r|}
\hline Property & Quantity & Diamond & \multicolumn{1}{c|}{ SF57 } \\
\hline Mechanical & $\mathrm{E}[\mathrm{Pa}]$ & $1.05 \cdot 10^{12}$ & \\
& $\sigma_{\mathrm{f}}[\mathrm{Pa}]$ & $3.75 \cdot 10^{9}$ & $1 \cdot 10^{7 *}$ \\
\hline Thermal & $\mathrm{k}[\mathrm{W} /(\mathrm{Km})]$ at $20\left[{ }^{\circ} \mathrm{C}\right]$ & 2000 & $0.15^{*}$ \\
& $\mathrm{k}[\mathrm{W} /(\mathrm{Km})]$ at $200\left[{ }^{\circ} \mathrm{C}\right]$ & 1200 & $1.50 *$ \\
\hline Optical & $\mathrm{n}[-]$ at $20\left[{ }^{\circ} \mathrm{C}\right]$ & 2.412 & 1.840 \\
& $\mathrm{n}[-]$ at $200\left[{ }^{\circ} \mathrm{C}\right]$ & 2.414 & 1.842 \\
& $\mathrm{dn} / \mathrm{dp}\left[\mathrm{Pa}^{-1}\right]$ & $8.68 \cdot 10^{-13}$ & $6 \cdot 10^{-14}$ \\
\hline
\end{tabular}

Table D.1: Properties of the window materials (Field [39] and Schott Glaswerke [130]). Values with * are estimated. E: Young's modulus, $\sigma_{\mathrm{f}}$ : fracture strength, k: thermal conductivity, $\mathrm{n}$ : refractive index and $\mathrm{dn} / \mathrm{dp}$ : pressure dependence of the refractive index.

Diamond has a high Young's modulus and a high thermal conductivity. The latter has an important effect on the temperature distribution within the cell. The refractive index is determined for the wavelength of the birefringence experiments $(632.8$ [nm], Schott Glaswerke [130] and Field [39]). The refractive index of both materials is quite high. Schott SF57 glass is more suitable for the optical measurements, because birefringence is less affected by stresses induced by flow and mounting. However, this 
material influences the temperature distribution within the sample more than the diamond material, as will be shown in the next section.

\section{D.2 Temperature gradients near window}

Temperature gradients near the windows are influenced by the thermal conductivity of the window material. The large difference in thermal conductivity (Table D.1) will show a different temperature distribution for diamond and SF57 glass. Information on heat transfer problems can be found in Bejan [11].

The diamond windows (type IIa; Drukker International, Cuijk, the Netherlands) have a thickness of $0.25[\mathrm{~mm}]$, a diameter of $4.2[\mathrm{~mm}]$, and are glued in a stainless steel frame (clear aperture $3.2[\mathrm{~mm}]$ ). The Schott SF windows have a thickness of $1.8[\mathrm{~mm}]$, a diameter of $8[\mathrm{~mm}]$, and are glued in a stainless steel frame (clear aperture $5.0[\mathrm{~mm}]$ ). The flow cell consists of construction steel (C45). The thermal conductivity of the materials used in the cell for two different temperatures are given in Table D.2. The conductivity of diamond decreases with temperature, it increases for the glass and it is more or less constant for the other materials. Ramsey III et al. [117] showed that the thermal conductivity of polypropylene is nearly independent of temperature.

\begin{tabular}{|l||r|r|r|}
\hline Material & $\begin{array}{c}\mathrm{k}[\mathrm{W} /(\mathrm{Km})] \\
\text { at } 20\left[{ }^{\circ} \mathrm{C}\right]\end{array}$ & $\begin{array}{c}\mathrm{k}[\mathrm{W} /(\mathrm{Km})] \\
\text { at } 200\left[{ }^{\circ} \mathrm{C}\right]\end{array}$ & Ref \\
\hline \hline Diamond & 2000 & 1200 & {$[39]$} \\
\hline Schott SF & 0.2 & 1.0 & {$[130]$} \\
\hline Stainless steel & 15 & 18 & {$[11]$} \\
\hline Steel & 54 & 48 & {$[11]$} \\
\hline Polypropylene & 0.2 & 0.2 & {$[117]$} \\
\hline
\end{tabular}

Table D.2: Thermal conductivity (k) for the materials used at two temperatures.

Since the windows are in contact with air, sample temperatures close to the windows could be lower than in the center of the cell. Finite elements calculations are performed to determine this deviation for both window materials used. The inner part of the flow cell is modeled as an axisymmetric problem for simplicity reasons. The geometries are given in Figure D.1. The position of the oil channel is at about $\mathrm{r}=12.5[\mathrm{~mm}]$, and the height and depth of the oil channel is equal to 3 and $5[\mathrm{~mm}]$, respectively.

The steady conduction problem with axisymmetric coordinates is described by

$$
k\left[\frac{1}{r} \frac{d}{d r}\left(r \frac{d T}{d r}\right)+\frac{d^{2} T}{d T^{2}}\right]=0
$$

Introducing dimensionless coordinates and temperature

$$
z^{*}=\frac{z}{L} ; \quad r^{*}=\frac{r}{L} ; \quad \theta=\frac{T-T_{\infty}}{T_{o}-T_{\infty}}
$$




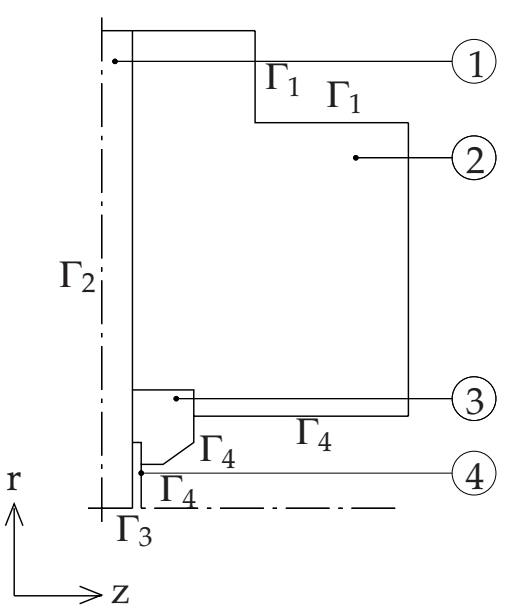

(a)

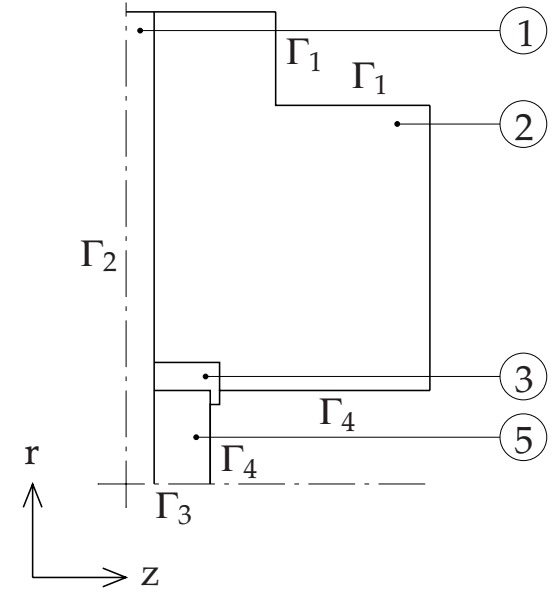

(b)

Figure D.1: 1. polymer, 2. steel, 3. window holder (stainless steel), 4. diamond window, 5. Schott SF57 window. Boundary conditions: oil temperature on $\Gamma_{1}$, symmetry on $\Gamma_{2}$ and $\Gamma_{3}$, and heat transfer on $\Gamma_{4}$.

with length $\mathrm{L}=1.0[\mathrm{~mm}]$, oil temperature $\mathrm{T}_{\mathrm{o}}=200\left[{ }^{\circ} \mathrm{C}\right]$ and air temperature $\mathrm{T}_{\infty}=50\left[{ }^{\circ} \mathrm{C}\right]$, gives

$$
k\left[\frac{1}{r^{*}} \frac{d}{d r^{*}}\left(r^{*} \frac{d \theta}{d r^{*}}\right)+\frac{d^{2} \theta}{d z^{* 2}}\right]=0
$$

The boundary conditions become

$$
\begin{aligned}
\theta & =1 & & \text { on } \Gamma_{1} \\
\frac{\partial \theta}{\partial z^{*}} & =0 & & \text { on } \Gamma_{2} \\
\frac{\partial \theta}{\partial r^{*}} & =0 & & \text { on } \Gamma_{3} \\
\frac{\partial \theta}{\partial n^{*}} & =-B i \theta & & \text { on } \Gamma_{4}
\end{aligned}
$$

with the Biot number $\mathrm{Bi}$, which is defined as

$$
B i=-\frac{h L}{k}
$$

with the conduction coefficient $\mathrm{k}[\mathrm{W} /(\mathrm{Km})]$ and the heat transfer coefficient $\mathrm{h}$ $\left[\mathrm{W} /\left(\mathrm{m}^{2} \mathrm{~K}\right)\right]$. The heat transfer coefficient for gases in case of natural convection is at most equal to $50\left[\mathrm{~W} /\left(\mathrm{m}^{2} \mathrm{~K}\right)\right]$. Boundary condition D.7 describes the heat transfer for the window, window holder and flow cell. Good heat transfer or a high Biot number is obtained for bad conducting materials. Consequently, diamond has a lower Biot number than the Schott SF57 glass. The Biot number for the materials used are given in Table D.3.

Linear triangular elements are used to model both geometries. The meshes are shown in Figure D.2. Natural boundary elements are used to describe the heat trans- 


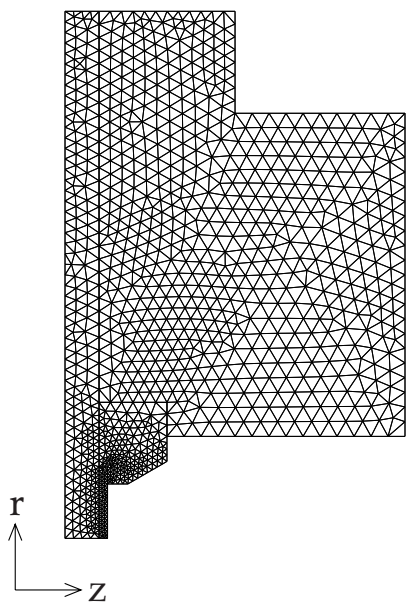

(a)

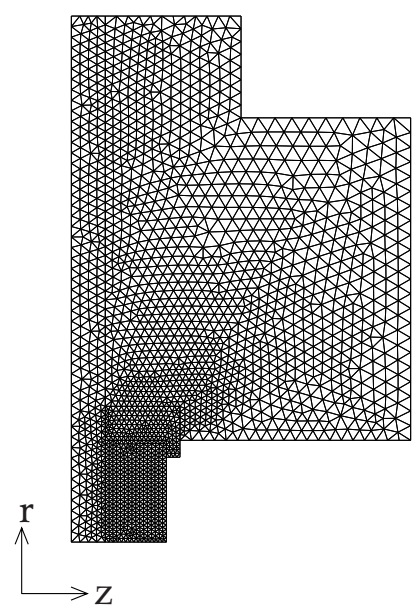

(b)

Figure D.2: (a) Mesh in case of diamond (2048 elements). (b) Mesh in case of Schott SF57 glass (3951 elements).

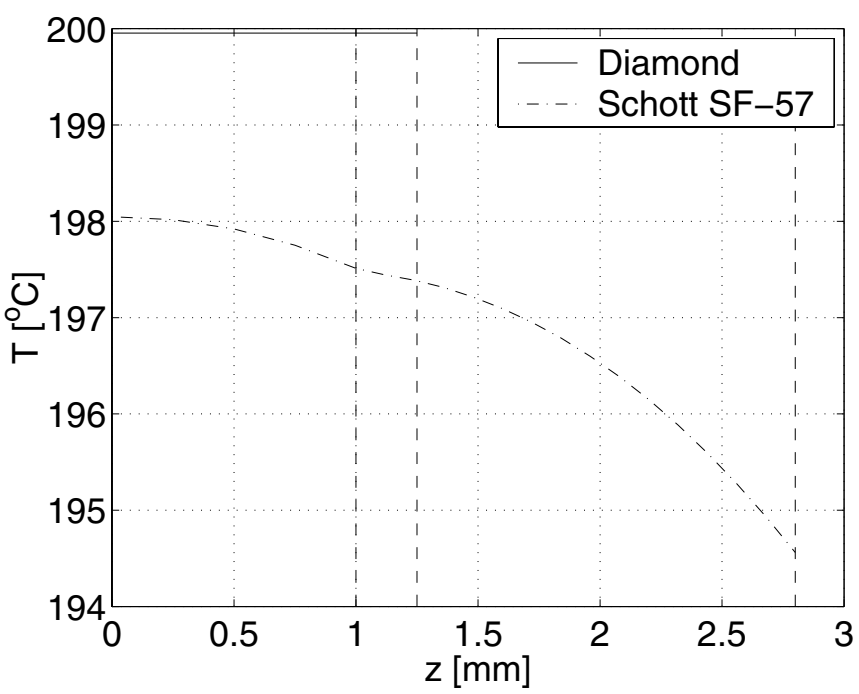

Figure D.3: Temperature distribution on $\mathrm{r}=0[\mathrm{~mm}]$. The vertical lines indicate the polymer channel, the diamond and SF57 glass window respectively. 


\begin{tabular}{|l||r|}
\hline material & $\mathrm{Bi}[-]$ at $200\left[{ }^{\circ} \mathrm{C}\right]$ \\
\hline \hline Diamond & $4.17 \cdot 10^{-5}$ \\
\hline Schott SF & $3.33 \cdot 10^{-2}$ \\
\hline Stainless steel & $2.78 \cdot 10^{-3}$ \\
\hline Steel & $1.04 \cdot 10^{-3}$ \\
\hline
\end{tabular}

Table D.3: Biot number for the materials used at $200\left[{ }^{\circ} \mathrm{C}\right]$.

fer at the window, stainless steel and construction steel boundary, and the material depending heat conduction coefficients are inserted. The temperature distribution is calculated using the Biot numbers at $200\left[{ }^{\circ} \mathrm{C}\right]$, and is shown for $\mathrm{r}=0[\mathrm{~mm}]$ in Figure D.3. Temperature gradients in the polymer channel can be neglected in case of the diamond windows. Temperature is about $0.05\left[{ }^{\circ} \mathrm{C}\right]$ below the the oil temperature.

Temperature gradients are much larger in case of SF57 glass due to the larger heat flow at the windows. The temperature in the polymer channel is about $2\left[{ }^{\circ} \mathrm{C}\right]$ lower than the oil temperature. Moreover, a gradient of about $0.5\left[{ }^{\circ} \mathrm{C}\right]$ in the depth of the flow channel can be observed. It can be concluded that diamond windows should be used to reduce thermal gradients. However, this material is not the best solution in case of birefringence studies.

\section{D.3 Influence of the refractive index on microscopy}

A stereo microscope together with a video camera are used to capture the field-wise birefringence images. The focusing of the microscope is not straight forward due to the different refractive indices of air, the window material and the polymer sample. In this section, the distance of the microscope to the flow cell is estimated.

The resolving power $\mathrm{d}$ of a microscope is defined by

$$
d=\frac{1.22 \lambda}{2 n \sin \alpha}
$$

with the half aperture angle $\alpha$, the refractive index of the immersing medium adjacent to the objective lens $\mathrm{n}$ and the wavelength $\lambda$. The resolving power and the focal distance $f$ of the microscope used (Zeiss, SV-11 with an achromatic lens of $0.63 \times$ ), are equal to $6.1[\mu \mathrm{m}]$ and $160[\mathrm{~mm}]$, respectively. Consequently, the half aperture angle is equal to 3.63 degrees (using the refractive index of air: $n=1[-]$ ).

The focusing of the microscope on the flow cell is drawn schematically in Figure D.4. The window thickness $x_{2}$ is equal to $1.8[\mathrm{~mm}]$ and half the sample thickness $x_{3}$ is equal to $1.0[\mathrm{~mm}]$. The thick line in Figure D.4 corresponds to the situation that $\mathrm{n}_{1}=\mathrm{n}_{3}=1$ [-] and $n_{2}>1[-]$. For the situation that $n_{3}>n_{2}$ (and $n_{1}=1[-]$ ) the focusing point moves further into the cell, while in case of $\mathrm{n}_{3}<\mathrm{n}_{2}$ it moves towards the window.

Using Snell's law at the two interfaces 1-2 and 2-3

$$
\begin{aligned}
& n_{2} \sin \theta_{2}=n_{1} \sin \theta_{1} \\
& n_{2} \sin \theta_{2}=n_{3} \sin \theta_{3}
\end{aligned}
$$




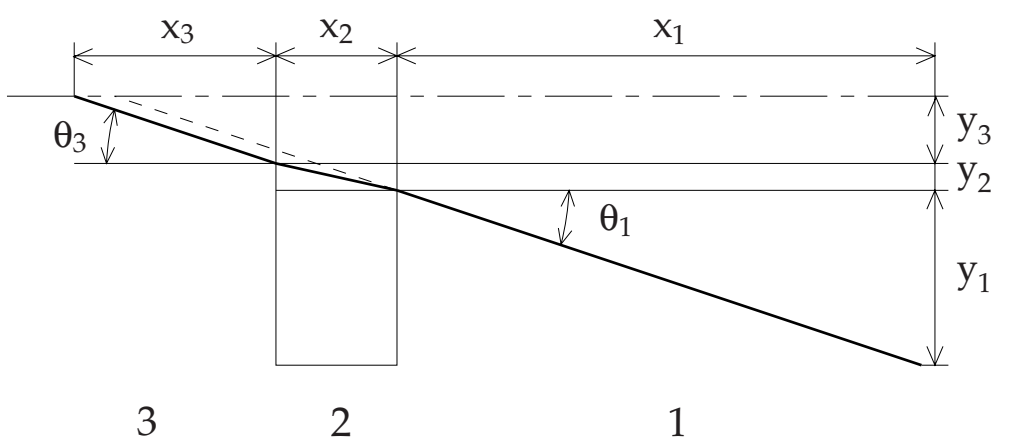

Figure D.4: Focusing of the microscope on the flow cell in the field-wise birefringent measurements. 1. air, 2. Schott SF57 glass window (thickness $1.8[\mathrm{~mm}]$ ), 3. polymer melt (iPP; thickness $1.0[\mathrm{~mm}]$ ).

the following two equations can be derived:

$$
\begin{aligned}
& \theta_{2}=\arcsin \left\{\frac{n_{1}}{n_{2}} \sin \theta_{1}\right\} \\
& \theta_{3}=\arcsin \left\{\frac{n_{1}}{n_{3}} \sin \theta_{1}\right\}
\end{aligned}
$$

The sum of the vertical distances $\mathrm{y}_{\text {tot }}$, using these equations, equals:

$$
\begin{aligned}
y_{\text {tot }} & =x_{1} \tan \theta_{1}+x_{2} \tan \theta_{2}+x_{3} \tan \theta_{3} \\
& =x_{1} \tan \theta_{1}+x_{2} \tan \left[\arcsin \left\{\frac{n_{1}}{n_{2}} \sin \theta_{1}\right\}\right]+x_{3} \tan \left[\arcsin \left\{\frac{n_{1}}{n_{3}} \sin \theta_{1}\right\}\right]
\end{aligned}
$$

The horizontal distance $\mathrm{x}_{1}$ between the microscopy and the window is calculated, using Equation (D.15). Three situations are distinguished in which the vertical distance $\mathrm{y}_{\text {tot }}$ is equal to $10.15[\mathrm{~mm}](\mathrm{f} \cdot \tan \alpha)$ :

1. $\mathrm{n}_{1}=\mathrm{n}_{2}=\mathrm{n}_{3}=1[-]$

$\mathrm{x}_{1}$ is equal to $157.2[\mathrm{~mm}]$.

2. $\mathrm{n}_{1}=\mathrm{n}_{3}=1[-]$, and $\mathrm{n}_{2}=1.84[-]$ (Schott SF57)

$\mathrm{x}_{1}$ is equal to 158.0 [mm].

3. $\mathrm{n}_{1}=1, \mathrm{n}_{2}=1.84$ [-] (Schott SF57) and $\mathrm{n}_{3}=1.45$ [-] (iPP [48])

$\mathrm{x}_{1}$ is equal to 158.4 [mm].

In conclusion, the difference between a cell with and without a polymer sample is about 0.4 [mm]. First, the microscope was aligned on a flow cell containing no sample and then moved back with an amount of $0.4[\mathrm{~mm}]$. 


\section{Samenvatting}

Eindeigenschappen van semi-kristallijne polymere producten hangen enerzijds af van de moleculaire parameters van het polymeer, maar via de zich in de stroming ontwikkelde structuur, ook van de verwerkingscondities toegepast tijdens het vormgevingsproces. Voor de beschrijving van het ontstaan en de groei van kiemen in afhankelijkheid van stroming, is modellering van de volledige thermische en mechanische voorgeschiedenis noodzakelijk.

In de literatuur beschreven experimenten laten zien dat stromingsgeïnduceerde kristallisatie correleert met de visco-elastische spanning die, tijdens het kristalliseren, in het product aanwezig is en niet met de lokale rek of reksnelheid. Het ontstaan en de groei van anisotrope, georiënteerde structuren wordt veroorzaakt doordat polymere ketens in de stroming worden georiënteerd en gerekt. De preciese dimensies en dimensie stabiliteit op korte en lange tijd na produceren van semi-kristallijne producten is bijgevolg slecht gedefinieerd. Kromtrekken van het product en (anisotrope) krimp zijn afhankelijk van de structuur en daardoor van de vorm van de matrijs en de verwerkingscondities. Om deze verschijnselen te voorspellen en om hiervoor te kunnen corrigeren zijn modellen nodig. Daartoe is in deze studie een gecombineerde numeriek-experimentele methode ontwikkeld, teneinde modellen te valideren en de bijbehorende modelparameters te bepalen.

Het doel van dit onderzoek is het onderzoeken van spanningsgeïnduceerde kristallisatie van polymere smelten in een goed gedefinieerde rekstroming, via het meten en voorspellen van de lokale structuurontwikkeling. Er is een experimentele stromingscel ontworpen, die geschikt is voor gebruik in de röntgenbundel van de Europese Synchrotron Stralingsfaciliteiten (ESRF) in Grenoble (Frankrijk). Daarnaast is numeriek gereedschap ontwikkeld om de structuurontwikkeling in de cel te beschrijven. Het Leonov model en het recentelijk ontwikkelde Pompom model zijn hiervoor gebruikt als basis.

Van twee typen polypropyleen (StamylanP 13E10 en 15M10 van DSM, Geleen), die verschillen in moleculair gewicht, zijn het stromings- en kristallisatiegedrag gekarakteriseerd. Om het thermo-reologisch gedrag, het relaxatietijdenspectrum en de niet lineaire parameters van het Pompom model te bepalen, is reologische karakterisering in zuivere afschuiving en uniaxiale rek gebruikt. Het Leonov model beschrijft het gedrag in afschuiving goed, maar geeft een slechte voorspelling in rek. Het Pompom model kan zowel de afschuivings- als de rekdata goed beschrijven. Uniaxiale rekexperimenten zijn echter lastig uit te voeren voor de gebruikte lineaire polymeren, vooral omdat het moleculair gewicht relatief laag is en de verwerkingstemperatuur hoog. 
Om het aantal nucleï en de groeisnelheid als functie van temperatuur te bepalen in afwezigheid van stroming, is een opstelling gebruikt waarin een microscoop gecombineerd wordt met een speciaal verwarmingsoventje. De groeisnelheden verschillen niet veel van elkaar, maar het aantal nuclei is veel groter in 13E10. De hogere kristallisatiesnelheid van 13E10 wordt voornamelijk hierdoor veroorzaakt. Vervolgens zijn de Schneidervergelijkingen gebruikt om de kristallisatiesnelheid in een DSC en in dynamische experimenten op een reometer te voorspellen. De temperaturen in de drie opstellingen (het verwarmingsoventje, de DSC en de reometer) zijn zorgvuldig gekalibreerd. Desalniettemin wordt er een afwijking gevonden in de kristallisatiesnelheid voor de gebruikte technieken.

Evenmin is er een unieke relatie gevonden tussen de toename van de modulus, als functie van de vulgraad, voor de verschillende isotherme-kristallisatie experimenten, voor beide materialen. Blijkbaar is deze relatie gevoelig voor experimentele fouten zoals het optreden van een transkristallisatie laag op het proefstukoppervlak. Deze laag is zichtbaar in de lichtmicroscoop.

In de stromingscel zijn optische dubbele-brekingsmetingen en grote hoek röntgenverstrooiingsmetingen uitgevoerd. Stroomlijnen van de voorafgaande stroming werden zichtbaar tijdens de dubbele-brekingsmetingen. Hun aanwezigheid wordt bevorderd door de onvermijdelijke afschuifsnelheid over de diepte van de stromingscel (3D effect). Een hoog georiënteerde structuur werd waargenomen tijdens de röntgenverstrooiingsmetingen. Uit de oriëntatie in de (110) en de (130) reflectie kon geconcludeerd worden dat de ontstane structuur geen lamellaire vertakkingen heeft en dat de afmetingen ongeveer $80[\mu \mathrm{m}]$ in de instroomrichting en minimaal $2[\mathrm{~mm}]$ in de stroomafwaartse richting bedragen.

Helaas werd er geen dominant effect van het stagnatiepunt gevonden zodra de stroming (ver) boven de smelttemperatuur plaatsvond, gevolgd door afkoeling naar kristallisatietemperatuur. Dit wordt veroorzaakt doordat ook afschuifgradiënten een grote invloed hebben in deze driedimensionale, complexe stroming.

Numerieke voorspellingen met het $S_{\mathrm{J} 2}$ model laten zien dat de invloed van afschuifgradiënten verminderd kan worden door een kortere stromingstijd te kiezen en/of door de stromingskanalen van de cel dieper te maken (grotere diepte/hoogte verhouding van het kanaal). Tevens moet de reproduceerbaarheid van de thermische geschiedenis in de cel verbeterd worden. De reproduceerbaarheid van de verplaatsing van de ring is goed, maar om grotere torsiekrachten over te kunnen brengen moet de opstelling veranderd worden. De grotere torsiekrachten zijn nodig om korte-duur experimenten, ook bij temperaturen onder de smelttemperatuur van het materiaal, uit te kunnen voeren. Tenslotte kan gesteld worden dat zowel de rekcomponent van de Finger tensor behorend bij de langste relaxatietijd, als het aantal stromingsgeïnduceerde nuclei, worden overschat door de te geprononceerde rekversteviging in het Leonov model. Het Pompom model presteert beter in complexe stromingen en wordt daarom aanbevolen als basis voor een kwantitatief stromingsgeïnduceerd kristallisatiemodel. 


\section{Acknowledgment}

The last four and a half years, I enjoyed the challenging research, which was a combination of numerical and experimental work. Due to this combination, time was limited to develop the software, and to perform all experiments. I owe many thanks to all the people who helped me during my research, and especially to:

Gerrit Peters (Special thanks for all those years of coaching!), Sanjay Rastogi and Han Meijer, for their enthusiastic coaching and discussions.

The people from our workshop, Sjef Garenfeld, Rob van den Berg, Toon van Gils and Karel Koekkoek, and Jan Ketelaars, Hans van de Westerlo and their colleagues of the central workshop (GTD), for their help and support.

The ESRF for using their facilities and their program 'fit2d', which has been developed by Andy Hammersley.

The people working at the micro-focus beam-line in Grenoble (ID13), Christian Riekel and Manfred Burghammer, and the at the materials beam-line in Grenoble (ID11), Ann Terry, for their help.

All people who helped with the X-ray experiments in Grenoble: Sanjay Rastogi, Gerrit Peters, Hans Zuidema, Ulrich Göschel, Hans Wilderbeek, Frank v/d Burgt, Bernard Schrauwen, Tosca Corstjens, Harold van Melick and Jan van Meerveld.

Annemarie Brouwers, Astrid Elzas, Judith Schuurman, Svetlana Ljevar and Maurice van Mensvoort for their characterization studies, and Elvira Somma.

Anne Spoelstra for the sample preparation of, and the morphological investigation on a DSC sample, and a quiescent and flow-induced rheometer sample, using light microscopy.

Markus Gahleitner (Borealis, Linz, Austria) for supplying the GPC and FTIR data, and Wim Zoetelief (DSM Research, Geleen, The Netherlands) for supplying the capillary rheometer and the uniaxial elongational data on DSM13E10 and DSM15M10.

Han Goossens (DSC), Hans Zuidema ( $\mathrm{S}_{\mathrm{J} 2}$ software and schneider visualization software), Wilco Verbeeten (viscoelastic constitutive equations), Patrick Anderson (spectral element code of SEPRAN), and the system managers Leo Wouters and Patrick van Brakel ('achter de kast').

Friends, room mates, colleagues, AntIlOpe, Asterix and many, many thanks to Marja and my family.

Frank Swartjes

Eindhoven, 17th September 2001 


\section{Curriculum Vitae}

1999-2001 Research Assistant at Materials Technology, Dutch Polymer Institute, Eindhoven University of Technology.

1997-1999 Computational Mechanics at the Stan Ackermans Institute (Institute for Continuing Education), Eindhoven University of Technology.

1992-1996 Biomechanical Engineering (post-propaedeutics program), Eindhoven University of Technology.

1991-1992 Mechanical Engineering, Eindhoven University of Technology.

1985-1991 Roman-Catholic comprehensive school, Canisiuscollege-Mater Dei (CCMD), Nijmegen.

19-04-1973 Born in Beuningen (Gld), the Netherlands. 